\title{
The effects of reversible addition fragmentation transfer (RAFT) on branching in vinyl acetate bulk polymerization
}

\author{
Supporting Information
}

\begin{abstract}
Mark A. Pinto*
Centre for Process Systems Engineering, Department of Chemical Engineering, Imperial College London, South Kensington Campus, London SW7 2AZ, UK.

and

School of Chemical and Biomolecular Engineering,

Georgia Institute of Technology, Atlanta GA 30332, USA

Rujun $\mathrm{Li}^{\dagger}$

School of Chemical and Biomolecular Engineering, Georgia Institute of Technology, Atlanta GA 30332, USA

Charles D. Immanuel

Centre for Process Systems Engineering, Department of Chemical Engineering, Imperial College London, South Kensington Campus, London SW7 2AZ, UK.
\end{abstract}

Peter A. Lovell

Materials Science Centre,

School of Materials,

The University of Manchester, Manchester M1 7HS, UK

\section{F. Joseph Schork ${ }^{\ddagger}$}

School of Chemical and Biomolecular Engineering, University of Maryland, College Park MD 20742, USA

*Ph.D. student at Imperial College London and visiting researcher at Georgia Tech

${ }^{\dagger}$ Current address: Corning Inc, Ithaca, NY, USA

${ }^{\ddagger}$ Corresponding author: F. Joseph Schork, e-mail: fjschork@umd.edu, Phone: +1 301405 1074, Fax: +1 3014050523 


\section{Contents}

1 RAFT Kinetic Mechanism S3

1.1 Notation . . . . . . . . . . . . . . . . . . . . . . . . . . . S3

1.2 Kinetics scheme . . . . . . . . . . . . . . . . . . . . . . . S3

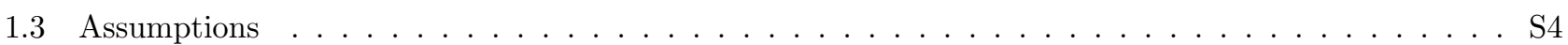

2 Mass balances $\quad$ S5

3 Derivation of moment mass balances $\quad \mathrm{S} 8$

3.1 Zeroth moment . . . . . . . . . . . . . . . . . . . . . . . . . . . . . . . . . . . . .

3.2 First moments . . . . . . . . . . . . . . . . . . . . . . . . . . . . . . . . . . . . . . . . . . . .

3.2.1 First moment w.r.t. the number of repeat units . . . . . . . . . . . . . . . . . S15

3.2.2 First moment w.r.t. the number of branches . . . . . . . . . . . . . . . . . . . . S21

3.2.3 First moment w.r.t. the number of active (uncapped) radicals . . . . . . . . . . . . . . S26

3.2.4 First moment w.r.t. the number of inactive (capped) radicals . . . . . . . . . . . . . . . . S33

3.2.5 First moment w.r.t. the number of unrelaxed (just uncapped) radicals . . . . . . . . . . . . S38

3.3 Second moments . . . . . . . . . . . . . . . . . . . . . . . . . . . S43

3.3.1 Second moment w.r.t. the number of repeat units . . . . . . . . . . . . . . . S43

3.3.2 Second moment w.r.t. the number of repeat units and active (uncapped) radicals . . . . . . . S49

3.3.3 Second moment w.r.t. the number of active (uncapped) radicals . . . . . . . . . . . . . . S56

3.3.4 Second moment w.r.t. the number of repeat units and unrelaxed (just uncapped) radicals . . S63

3.3.5 Second moment w.r.t. the number of active (uncapped) radicals and unrelaxed (just uncapped) radicals . . . . . . . . . . . . . . . . . . . . . . . . S68

3.3.6 Second moment w.r.t. the number of repeat units and inactive (capped) radicals . . . . . . S75

3.3.7 Second moment w.r.t. the number of unrelaxed (just uncapped) radicals . . . . . . . . . . . . S81

3.3.8 Second moment w.r.t. the number of active (uncapped) radicals and inactive (capped) radicalsS87

3.3.9 Second moment w.r.t. the number of inactive (capped) radicals and unrelaxed (just uncapped) radicals . . . . . . . . . . . . . . . . . . . . . . . . . . . . . . . . . . . . . . . . . . . . . . . . . . . .

3.3.10 Second moment w.r.t. the number of inactive (capped) radicals . . . . . . . . . . . . . . S97 


\section{RAFT Kinetic Mechanism}

\subsection{Notation}

Polymer chains are represented by $P_{i j k l m}$ where

$i$ represents the number of repeat units,

$j$ represents the number of branches,

$k$ represents the number of active (uncapped) radicals,

$l$ represents the number of inactive (capped) radicals, and

$m$ represents the number of unrelaxed (just uncapped) radicals.

\subsection{Kinetics scheme}

Initiator decomposition

$$
I \stackrel{k_{d}}{\rightarrow} 2 R
$$

Initiation

$$
R+M \stackrel{k_{i}}{\rightarrow} P_{10100}
$$

Propagation

$$
\begin{array}{ll}
P_{i j k l m}+M \stackrel{k_{p}}{\rightarrow} P_{i+1 j k l m} & k>0 \\
P_{i j k l m}+M \stackrel{k_{p}}{\rightarrow} P_{i+1 j k l m} & m>0
\end{array}
$$

RAFT Transfer

$$
\begin{aligned}
& T+P_{i j k l m} \stackrel{k_{t r t}}{\rightarrow} P_{i j k-1 l+1 m}+R \\
& P_{i_{1} j_{1} k_{1} l_{1} m_{1}}+P_{i_{2} j_{2} k_{2} l_{2} m_{2}} \stackrel{k_{t r t}}{\longrightarrow} P_{i_{1} j_{1} k_{1}-1 l_{1}+1 m_{1}}+P_{i_{2} j_{2} k_{2} l_{2}-1 m_{2}+1}
\end{aligned}
$$

Intermolecular chain transfer

$$
\begin{aligned}
& P_{i_{1} j_{1} k_{1} l_{1} m_{1}}+P_{i_{2} j_{2} k_{2} l_{2} m_{2}} \stackrel{k_{\text {trp }}}{\longrightarrow} P_{i_{1} j_{1} k_{1}-1 l_{1} m_{1}}+P_{i_{2} j_{2}+1 k_{2}+1 l_{2} m_{2}} \\
& P_{i_{1} j_{1} k_{1} l_{1} m_{1}}+P_{i_{2} j_{2} k_{2} l_{2} m_{2}} \stackrel{k_{t r p}}{\longrightarrow} P_{i_{1} j_{1} k_{1} l_{1} m_{1}-1}+P_{i_{2} j_{2}+1 k_{2} l_{2} m_{2}+1}
\end{aligned}
$$

Intramolecular chain transfer

$$
P_{i j k l m} \stackrel{k_{\text {intr }}}{\longrightarrow} P_{i j+1 k l m}
$$

Relaxation

$$
P_{i j k l m} \stackrel{k_{r}}{\rightarrow} P_{i j k+1 l m-1}
$$

Termination by combination

$$
\begin{aligned}
& P_{i_{1} j_{1} k_{1} l_{1} m_{1}}+P_{i_{2} j_{2} k_{2} l_{2} m_{2}} \stackrel{k_{t c}}{\longrightarrow} P_{i_{1}+i_{2}, j_{1}+j_{2}, k_{1}+k_{2}-2, l_{1}+l_{2}, m_{1}+m_{2}} \\
& P_{i_{1} j_{1} k_{1} l_{1} m_{1}}+P_{i_{2} j_{2} k_{2} l_{2} m_{2}} \stackrel{k_{t c}}{\longrightarrow} P_{i_{1}+i_{2}, j_{1}+j_{2}, k_{1}+k_{2}, l_{1}+l_{2}, m_{1}+m_{2}-2} \\
& P_{i_{1} j_{1} k_{1} l_{1} m_{1}}+P_{i_{2} j_{2} k_{2} l_{2} m_{2}} \stackrel{k_{t c}}{\longrightarrow} P_{i_{1}+i_{2}, j_{1}+j_{2}, k_{1}+k_{2}-1, l_{1}+l_{2}, m_{1}+m_{2}-1}
\end{aligned}
$$




\subsection{Assumptions}

1. In the propagation reaction Equation (3), the rate of the reaction is proportional to (a) the concentration of monomer and (b) the number of active (uncapped) radicals in the polymer chain. Similarly, in the propagation reaction Equation (4), the rate of the reaction is proportional to (a) the concentration of monomer and (b) the number of (just uncapped) unrelaxed radicals in the polymer chain.

2. The propagation reactions Equations (3) and (4) have the same rate constant, $k_{p}$.

3. The rate of propagation from a tertiary radical produced by either intermolecular and intramolecular chain transfer is much lower than from a 'normal' chain-end radical. However, the rate constant for propagation from all radicals has been assumed to be the same in order to maintain simplicity.

4. In the RAFT transfer reaction Equation (5), the rate of the reaction is proportional to (a) the concentration of the RAFT agent and (b) the number of active (uncapped) radicals in the polymer chain to which the RAFT agent is being transferred.

5. In the RAFT transfer reaction Equation (6), the rate of the reaction is proportional to the (a) the number of active (uncapped) radicals in the polymer chain to which the RAFT agent is being transferred, and (b) the number of inactive (capped) radicals in the polymer chain from which the RAFT agent is being transferred.

6. In the intermolecular chain transfer to polymer reaction Equation (7), the rate of the reaction is proportional to (a) the number of repeat units in the polymer receiving the chain, and (b) the number of active (uncapped) radicals in the polymer from which the chain is being transferred. Similarly, in the intermolecular chain transfer reaction to polymer Equation (8), the rate of the reaction is proportional to (a) the number of repeat units in the polymer receiving the chain, and (b) the number of unrelaxed (just uncapped) radicals in the polymer from which the chain is being transferred.

7. The intermolecular chain transfer to polymer reactions Equations (7) and (8) have the same rate constant, $k_{t r p}$.

8. In the intramolecular chain transfer to polymer reaction Equation (9) the rate of the reaction is proportional to the number of active radicals in the polymer chain.

9. In the relaxation reaction Equation (10), the rate of the reaction is proportional to the number of unrelaxed radicals in the polymer chain undergoing relaxation.

10. In the termination by combination reaction Equation (11), the rate of the reaction is proportional to the number of active (uncapped) radicals in the polymer chains participating in the reaction. Similarly, in the termination by combination reaction Equation (12), the rate of the reaction is proportional to the number of unrelaxed (just uncapped) radicals in the polymer chains participating in the reaction.

11. In the termination by combination reaction Equation (13), the rate of the reaction is proportional to (a) the number of active (uncapped) radicals in the first polymer chain and (b) the number of unrelaxed (just uncapped) radicals in the second polymer chain participating in the reaction.

12. The termination by combination reactions Equations $(11),(12)$ and $(13)$ have the same rate constant, $k_{t c}$. 


\section{Mass balances}

Initiator

$$
\frac{d C_{I}}{d t}=-k_{d} C_{I}
$$

Free radicals

$$
\frac{d C_{R}}{d t}=2 k_{d} C_{I}-k_{i} C_{M} C_{R}+k_{t r t} C_{T} \mu_{00100}
$$

Monomer

$$
\frac{d C_{M}}{d t}=-k_{i} C_{M} C_{R}-k_{p} C_{M} \mu_{00100}-k_{p} C_{M} \mu_{00001}
$$

\section{RAFT Agent}

$$
\frac{d C_{T}}{d t}=-k_{t r t} C_{T} \mu_{00100}
$$

Zeroth moment

$$
\frac{d \mu_{00000}}{d t}=k_{i} C_{M} C_{R}-\frac{1}{2} k_{t c}\left(\mu_{00100}\right)^{2}-\frac{1}{2} k_{t c}\left(\mu_{00001}\right)^{2}-k_{t c} \mu_{00100} \mu_{00001}
$$

First moment w.r.t. the number of repeat units

$$
\frac{d \mu_{10000}}{d t}=k_{i} C_{M} C_{R}+k_{p} C_{M} \mu_{00100}+k_{p} C_{M} \mu_{00001}
$$

First moment w.r.t. the number of branches

$$
\frac{d \mu_{01000}}{d t}=k_{t r p} \mu_{10000} \mu_{00100}+k_{t r p} \mu_{10000} \mu_{00001}+k_{i n t r} \mu_{00100}
$$

First moment w.r.t. the number of active (uncapped) radicals

$$
\begin{aligned}
\frac{d \mu_{00100}}{d t} & =k_{i} C_{M} C_{R}-k_{t r t} C_{T} \mu_{00100}-k_{t r t} \mu_{00100} \mu_{00010}+k_{r} \mu_{00001} \\
& -k_{t c}\left(\mu_{00100}\right)^{2}-k_{t c} \mu_{00100} \mu_{00001}
\end{aligned}
$$

First moment w.r.t. the number of inactive (capped) radicals

$$
\frac{d \mu_{000010}}{d t}=k_{t r t} C_{T} \mu_{00100}
$$

First moment w.r.t. the number of unrelaxed (just uncapped) radicals

$$
\frac{d \mu_{00001}}{d t}=k_{t r t} \mu_{00010} \mu_{00100}-k_{r} \mu_{00001}-k_{t c}\left(\mu_{00001}\right)^{2}-k_{t c} \mu_{00100} \mu_{00001}
$$

Second moment w.r.t. the number of repeat units

$$
\begin{aligned}
\frac{d \mu_{20000}}{d t} & =k_{i} C_{M} C_{R}+k_{p} C_{M}\left(2 \mu_{10100}+\mu_{00100}\right)+k_{p} C_{M}\left(2 \mu_{10001}+\mu_{00001}\right)+k_{t c}\left(\mu_{10100}\right)^{2} \\
& +k_{t c}\left(\mu_{10001}\right)^{2}+2 k_{t c} \mu_{10100} \mu_{10001}
\end{aligned}
$$


Second moment w.r.t. the number of repeat units and active (uncapped) radicals

$$
\begin{aligned}
\frac{d \mu_{10100}}{d t} & =k_{i} C_{M} C_{R}+k_{p} C_{M} \mu_{00200}+k_{p} C_{M} \mu_{00101}-k_{f i} C_{I} \mu_{10100}-k_{t r t} C_{T} \mu_{10100} \\
& -k_{t r t} \mu_{10100} \mu_{00010}-k_{t r p} \mu_{10100} \mu_{10000}+k_{t r p} \mu_{20000} \mu_{00100}+k_{r} \mu_{10001} \\
& -k_{t c} \mu_{10100} \mu_{00100}-\frac{1}{2} k_{t c} \mu_{10100} \mu_{00001}-\frac{1}{2} k_{t c} \mu_{00100} \mu_{10001}
\end{aligned}
$$

Second moment w.r.t. the number of active (uncapped) radicals

$$
\begin{aligned}
\frac{d \mu_{00200}}{d t} & =k_{i} C_{M} C_{R}+k_{t r t} C_{T}\left(\mu_{00100}-2 \mu_{00200}\right) \\
& +k_{t r t} \mu_{00010}\left(\mu_{00100}-2 \mu_{00200}\right)+k_{t r p} \mu_{10000}\left(\mu_{00100}-2 \mu_{00200}\right)+k_{t r p} \mu_{00100}\left(2 \mu_{10100}+\mu_{10000}\right) \\
& +k_{r}\left(2 \mu_{00101}+\mu_{00001}\right)+k_{t c}\left[\left(\mu_{00200}\right)^{2}-4 \mu_{00200} \mu_{00100}+2\left(\mu_{00100}\right)^{2}\right] \\
& +2 k_{t c} \mu_{00200}\left(\mu_{00101}-\mu_{00001}\right)+k_{t c} \mu_{00100}\left(\mu_{00001}-2 \mu_{00101}\right)
\end{aligned}
$$

Second moment w.r.t. the number of repeat units and unrelaxed (just uncapped) radicals

$$
\begin{aligned}
\frac{d \mu_{10001}}{d t} & =k_{p} C_{M} \mu_{00101}+k_{p} C_{M} \mu_{00002}+k_{t r t} \mu_{10010} \mu_{00100}-k_{t r p} \mu_{10001} \mu_{10000}+k_{t r p} \mu_{20000} \mu_{00001} \\
& -k_{r} \mu_{10001}-k_{t c} \mu_{10001} \mu_{00001}-\frac{1}{2} k_{t c} \mu_{10100} \mu_{00001}-\frac{1}{2} k_{t c} \mu_{10001} \mu_{00100}
\end{aligned}
$$

Second moment w.r.t. the number of active (uncapped) radicals and unrelaxed (just uncapped) radicals

$$
\begin{aligned}
\frac{d \mu_{00101}}{d t} & =-k_{t r t} C_{T} \mu_{00101}-k_{t r t} \mu_{00101} \mu_{00010}+k_{t r t} \mu_{00110} \mu_{00100} \\
& -2 k_{t r p} \mu_{00101} \mu_{10000}+k_{t r p} \mu_{10001} \mu_{00100}+k_{t r p} \mu_{10100} \mu_{00001}+k_{r}\left(\mu_{00002}-\mu_{00101}-\mu_{00001}\right) \\
& +k_{t c} \mu_{00100} \mu_{00001}-\frac{3}{2} k_{t c} \mu_{00101}\left(\mu_{00100}+\mu_{00001}\right)-\frac{1}{2} k_{t c} \mu_{00200} \mu_{00001}-\frac{1}{2} k_{t c} \mu_{00002} \mu_{00100}
\end{aligned}
$$

Second moment w.r.t. the number of repeat units and inactive (capped) radicals

$$
\begin{aligned}
\frac{d \mu_{10010}}{d t} & =k_{p} C_{M} \mu_{00110}+k_{p} C_{M} \mu_{00011}+k_{t r t} C_{T} \mu_{10100}+k_{t r t} \mu_{10100} \mu_{00010} \\
& -k_{t r t} \mu_{10010} \mu_{00100}
\end{aligned}
$$

Second moment w.r.t the number of unrelaxed (just uncapped) radicals

$$
\begin{aligned}
\frac{d \mu_{00002}}{d t} & =k_{t r t} \mu_{00100}\left(2 \mu_{00011}+\mu_{00010}\right)+k_{t r p}\left(\mu_{00001}-2 \mu_{00002}\right) \mu_{10000} \\
& +k_{t r p}\left(2 \mu_{10001}+\mu_{10000}\right) \mu_{00001}-k_{r}\left(2 \mu_{00002}-\mu_{00001}\right) \\
& +k_{t c}\left[\left(\mu_{00002}\right)^{2}-4 \mu_{00002} \mu_{00001}+2\left(\mu_{00001}\right)^{2}\right] \\
& +2 k_{t c} \mu_{00002}\left(\mu_{00101}-\mu_{00100}\right)+k_{t c} \mu_{00001}\left(\mu_{00100}-2 \mu_{00101}\right)
\end{aligned}
$$

Second moment w.r.t the number of active (uncapped) radicals and inactive (capped) radicals

$$
\begin{aligned}
\frac{d \mu_{00110}}{d t} & =k_{t r t}\left(C_{T}+\mu_{00010}\right)\left(\mu_{00200}-\mu_{00110}-\mu_{00100}\right) \\
& -k_{t r t} \mu_{00100} \mu_{00110}-k_{t r p} \mu_{00110} \mu_{10000}+k_{t r p} \mu_{10010} \mu_{00100}+k_{r} \mu_{00011} \\
& -k_{t c} \mu_{00110} \mu_{00100}-\frac{1}{2} \mu_{00110} \mu_{00001}-\frac{1}{2} \mu_{00011} \mu_{00100}
\end{aligned}
$$


Second moment w.r.t the number of inactive (uncapped) radicals and unrelaxed (just uncapped) radicals

$$
\begin{aligned}
& \frac{d \mu_{00011}}{d t}=k_{t r t}\left(C_{T}+\mu_{00010}\right) \mu_{00101}+k_{t r t} \mu_{00100}\left(\mu_{00020}-\mu_{00011}-\mu_{00001}\right) \\
& -k_{t r p} \mu_{00011} \mu_{10000}+k_{t r p} \mu_{10010} \mu_{00001}-k_{r} \mu_{00011} \\
& -k_{t c} \mu_{00110} \mu_{00001}-\frac{1}{2} k_{t c} \mu_{00110} \mu_{00001}-\frac{1}{2} k_{t c} \mu_{00011} \mu_{00100}
\end{aligned}
$$

Second moment w.r.t the number of inactive (capped) radicals

$$
\begin{gathered}
\frac{d \mu_{00020}}{d t}=k_{t r t}\left(C_{T}+\mu_{00010}\right)\left(2 \mu_{00110}+\mu_{00100}\right)+k_{t r t} \mu_{00100}\left(\mu_{00010}-2 \mu_{00020}\right) \\
+k_{t c}\left(\mu_{00110}\right)^{2}+k_{t c}\left(\mu_{00011}\right)^{2}+2 k_{t c} \mu_{00110} \mu_{00011}
\end{gathered}
$$




\section{Derivation of moment mass balances}

$$
\begin{aligned}
& \frac{d P_{10100}}{d t}=k_{i} C_{M} C_{R}+\ldots \\
& \frac{d P_{i j k l m}}{d t}=k_{p} C_{M} k\left(-P_{i j k l m}+P_{i-1 j k l m}\right) \\
& +k_{p} C_{M} m\left(-P_{i j k l m}+P_{i-1 j k l m}\right) \\
& +k_{t r t} C_{T}\left(-k P_{i j k l m}+(k+1) P_{i j k+1 l-1 m}\right) \\
& -k_{t r t} k P_{i j k l m} \sum_{i_{1}=1}^{\infty} \sum_{j_{1}=0}^{\infty} \sum_{k_{1}=0}^{\infty} \sum_{l_{1}=0}^{\infty} \sum_{m_{1}=0}^{\infty} l_{1} P_{i_{1} j_{1} k_{1} l_{1} m_{1}} \\
& -k_{t r t} l P_{i j k l m} \sum_{i_{1}=1}^{\infty} \sum_{j_{1}=0}^{\infty} \sum_{k_{1}=0}^{\infty} \sum_{l_{1}=0}^{\infty} \sum_{m_{1}=0}^{\infty} k_{1} P_{i_{1} j_{1} k_{1} l_{1} m_{1}} \\
& +k_{t r t}(k+1) P_{i j k+1 l-1 m} \sum_{i_{1}=1}^{\infty} \sum_{j_{1}=0}^{\infty} \sum_{k_{1}=0}^{\infty} \sum_{l_{1}=0}^{\infty} \sum_{m_{1}=0}^{\infty} l_{1} P_{i_{1} j_{1} k_{1} l_{1} m_{1}} \\
& +k_{t r t}(l+1) P_{i j k l+1 m-1} \sum_{i_{1}=1}^{\infty} \sum_{j_{1}=0}^{\infty} \sum_{k_{1}=0}^{\infty} \sum_{l_{1}=0}^{\infty} \sum_{m_{1}=0}^{\infty} k_{1} P_{i_{1} j_{1} k_{1} l_{1} m_{1}} \\
& -k_{t r p} k P_{i j k l m} \sum_{i_{1}=1}^{\infty} \sum_{j_{1}=0}^{\infty} \sum_{k_{1}=0}^{\infty} \sum_{l_{1}=0}^{\infty} \sum_{m_{1}=0}^{\infty} i_{1} P_{i_{1} j_{1} k_{1} l_{1} m_{1}} \\
& -k_{t r p} i P_{i j k l m} \sum_{i_{1}=1}^{\infty} \sum_{j_{1}=0}^{\infty} \sum_{k_{1}=0}^{\infty} \sum_{l_{1}=0}^{\infty} \sum_{m_{1}=0}^{\infty} k_{1} P_{i_{1} j_{1} k_{1} l_{1} m_{1}} \\
& +k_{t r p}(k+1) P_{i j k+1 l m} \sum_{i_{1}=1}^{\infty} \sum_{j_{1}=0}^{\infty} \sum_{k_{1}=0}^{\infty} \sum_{l_{1}=0}^{\infty} \sum_{m_{1}=0}^{\infty} i_{1} P_{i_{1} j_{1} k_{1} l_{1} m_{1}} \\
& +k_{t r p} i P_{i j-1 k-1 l m} \sum_{i_{1}=1}^{\infty} \sum_{j_{1}=0}^{\infty} \sum_{k_{1}=0}^{\infty} \sum_{l_{1}=0}^{\infty} \sum_{m_{1}=0}^{\infty} k_{1} P_{i_{1} j_{1} k_{1} l_{1} m_{1}} \\
& -k_{t r p} m P_{i j k l m} \sum_{i_{1}=1}^{\infty} \sum_{j_{1}=0}^{\infty} \sum_{k_{1}=0}^{\infty} \sum_{l_{1}=0}^{\infty} \sum_{m_{1}=0}^{\infty} i_{1} P_{i_{1} j_{1} k_{1} l_{1} m_{1}} \\
& -k_{t r p} i P_{i j k l m} \sum_{i_{1}=1}^{\infty} \sum_{j_{1}=0}^{\infty} \sum_{k_{1}=0}^{\infty} \sum_{l_{1}=0}^{\infty} \sum_{m_{1}=0}^{\infty} m_{1} P_{i_{1} j_{1} k_{1} l_{1} m_{1}} \\
& +k_{t r p}(m+1) P_{i j k l m+1} \sum_{i_{1}=1}^{\infty} \sum_{j_{1}=0}^{\infty} \sum_{k_{1}=0}^{\infty} \sum_{l_{1}=0}^{\infty} \sum_{m_{1}=0}^{\infty} i_{1} P_{i_{1} j_{1} k_{1} l_{1} m_{1}} \\
& +k_{t r p} i P_{i j-1 k l m-1} \sum_{i_{1}=1}^{\infty} \sum_{j_{1}=0}^{\infty} \sum_{k_{1}=0}^{\infty} \sum_{l_{1}=0}^{\infty} \sum_{m_{1}=0}^{\infty} m_{1} P_{i_{1} j_{1} k_{1} l_{1} m_{1}} \\
& +k_{i n t r} k\left(-P_{i j k l m}+P_{i j-1 k l m}\right) \\
& +k_{r}\left[-m P_{i j k l m}+(m+1) P_{i j k-1 l m+1}\right]
\end{aligned}
$$




$$
\begin{aligned}
& -k_{t c} k P_{i j k l m} \sum_{i_{1}=1}^{\infty} \sum_{j_{1}=0}^{\infty} \sum_{k_{1}=0}^{\infty} \sum_{l_{1}=0}^{\infty} \sum_{m_{1}=0}^{\infty} k_{1} P_{i_{1} j_{1} k_{1} l_{1} m_{1}} \\
& +\frac{1}{2} k_{t c} \sum_{i_{1}=1}^{i} \sum_{j_{1}=0}^{j} \sum_{k_{1}=0}^{k+2} \sum_{l_{1}=0}^{l} \sum_{m_{1}=0}^{m}\left[k_{1} P_{i_{1} j_{1} k_{1} l_{1} m_{1}}\left(k+2-k_{1}\right) P_{\left.i-i_{1}, j-j_{1}, k+2-k_{1}, l-l_{1} m-m_{1}\right]}\right] \\
& -k_{t c} m P_{i j k l m} \sum_{i_{1}=1}^{\infty} \sum_{j_{1}=0}^{\infty} \sum_{k_{1}=0}^{\infty} \sum_{l_{1}=0}^{\infty} \sum_{m_{1}=0}^{\infty} m_{1} P_{i_{1} j_{1} k_{1} l_{1} m_{1}} \\
& +\frac{1}{2} k_{t c} \sum_{i_{1}=1}^{i} \sum_{j_{1}=0}^{j} \sum_{k_{1}=0}^{k} \sum_{l_{1}=0}^{l} \sum_{m_{1}=0}^{m+2}\left[m_{1} P_{i_{1} j_{1}} k_{1} l_{1} m_{1}\left(m+2-m_{1}\right) P_{\left.i-i_{1}, j-j_{1}, k-k_{1}, l-l_{1} m+2-m_{1}\right]}\right] \\
& -k_{t c} k P_{i j k l m} \sum_{i_{1}=1}^{\infty} \sum_{j_{1}=0}^{\infty} \sum_{k_{1}=0}^{\infty} \sum_{l_{1}=0}^{\infty} \sum_{m_{1}=0}^{\infty} m_{1} P_{i_{1} j_{1} k_{1} l_{1} m_{1}} \\
& -k_{t c} m P_{i j k l m} \sum_{i_{1}=1}^{\infty} \sum_{j_{1}=0}^{\infty} \sum_{k_{1}=0}^{\infty} \sum_{l_{1}=0}^{\infty} \sum_{m_{1}=0}^{\infty} k_{1} P_{i_{1} j_{1} k_{1} l_{1} m_{1}} \\
& +\frac{1}{2} k_{t c} \sum_{i_{1}=1}^{i} \sum_{j_{1}=0}^{j} \sum_{k_{1}=0}^{k+1} \sum_{l_{1}=0}^{l} \sum_{m_{1}=0}^{m+1}\left[k_{1} P_{i_{1} j_{1}} k_{1} l_{1} m_{1}\left(m+1-m_{1}\right) P_{\left.i-i_{1}, j-j_{1}, k+1-k_{1}, l-l_{1} m+1-m_{1}\right]}\right] \\
& +\frac{1}{2} k_{t c} \sum_{i_{1}=1}^{i} \sum_{j_{1}=0}^{j} \sum_{k_{1}=0}^{k+1} \sum_{l_{1}=0}^{l} \sum_{m_{1}=0}^{m+1}\left[m_{1} P_{i_{1} j_{1} k_{1} l_{1} m_{1}}\left(k+1-k_{1}\right) P_{\left.i-i_{1}, j-j_{1}, k+1-k_{1}, l-l_{1} m+1-m_{1}\right]}\right.
\end{aligned}
$$




\subsection{Zeroth moment}

$$
\begin{aligned}
& \frac{d \mu_{00000}}{d t}=\sum_{i=1}^{\infty} \sum_{j=0}^{\infty} \sum_{k=0}^{\infty} \sum_{l=0}^{\infty} \sum_{m=0}^{\infty} \frac{d P_{i j k l m}}{d t} \\
& =k_{i} C_{M} C_{R} \\
& -k_{p} C_{M} \sum_{i=1}^{\infty} \sum_{j=0}^{\infty} \sum_{k=0}^{\infty} \sum_{l=0}^{\infty} \sum_{m=0}^{\infty} k P_{i j k l m} \\
& +k_{p} C_{M} \sum_{i=2}^{\infty} \sum_{j=0}^{\infty} \sum_{k=0}^{\infty} \sum_{l=0}^{\infty} \sum_{m=0}^{\infty} k P_{i-1 j k l m} \\
& -k_{p} C_{M} \sum_{i=1}^{\infty} \sum_{j=0}^{\infty} \sum_{k=0}^{\infty} \sum_{l=0}^{\infty} \sum_{m=0}^{\infty} m P_{i j k l m} \\
& +k_{p} C_{M} \sum_{i=2}^{\infty} \sum_{j=0}^{\infty} \sum_{k=0}^{\infty} \sum_{l=0}^{\infty} \sum_{m=0}^{\infty} m P_{i-1 j k l m} \\
& -k_{t r t} C_{T} \sum_{i=1}^{\infty} \sum_{j=0}^{\infty} \sum_{k=0}^{\infty} \sum_{l=0}^{\infty} \sum_{m=0}^{\infty} k P_{i j k l m} \\
& +k_{t r t} C_{T} \sum_{i=1}^{\infty} \sum_{j=0}^{\infty} \sum_{k=0}^{\infty} \sum_{l=1}^{\infty} \sum_{m=0}^{\infty}(k+1) P_{i j k+1 l-1 m} \\
& -k_{t r t} \sum_{i=1}^{\infty} \sum_{j=0}^{\infty} \sum_{k=0}^{\infty} \sum_{l=0}^{\infty} \sum_{m=0}^{\infty} k P_{i j k l m} \sum_{i_{1}=1}^{\infty} \sum_{j_{1}=0}^{\infty} \sum_{k_{1}=0}^{\infty} \sum_{l_{1}=0}^{\infty} \sum_{m_{1}=0}^{\infty} l_{1} P_{i_{1} j_{1} k_{1} l_{1} m_{1}} \\
& -k_{\text {trt }} \sum_{i=1}^{\infty} \sum_{j=0}^{\infty} \sum_{k=0}^{\infty} \sum_{l=0}^{\infty} \sum_{m=0}^{\infty} l P_{i j k l m} \sum_{i_{1}=1}^{\infty} \sum_{j_{1}=0}^{\infty} \sum_{k_{1}=0}^{\infty} \sum_{l_{1}=0}^{\infty} \sum_{m_{1}=0}^{\infty} k_{1} P_{i_{1} j_{1} k_{1} l_{1} m_{1}} \\
& +k_{t r t} \sum_{i=1}^{\infty} \sum_{j=0}^{\infty} \sum_{k=0}^{\infty} \sum_{l=1}^{\infty} \sum_{m=0}^{\infty}(k+1) P_{i j k+1 l-1 m} \sum_{i_{1}=1}^{\infty} \sum_{j_{1}=0}^{\infty} \sum_{k_{1}=0}^{\infty} \sum_{l_{1}=0}^{\infty} \sum_{m_{1}=0}^{\infty} l_{1} P_{i_{1} j_{1} k_{1} l_{1} m_{1}} \\
& +k_{t r t} \sum_{i=1}^{\infty} \sum_{j=0}^{\infty} \sum_{k=0}^{\infty} \sum_{l=0}^{\infty} \sum_{m=1}^{\infty}(l+1) P_{i j k l+1 m-1} \sum_{i_{1}=1}^{\infty} \sum_{j_{1}=0}^{\infty} \sum_{k_{1}=0}^{\infty} \sum_{l_{1}=0}^{\infty} \sum_{m_{1}=0}^{\infty} k_{1} P_{i_{1} j_{1} k_{1} l_{1} m_{1}} \\
& -k_{t r p} \sum_{i=1}^{\infty} \sum_{j=0}^{\infty} \sum_{k=0}^{\infty} \sum_{l=0}^{\infty} \sum_{m=0}^{\infty} k P_{i j k l m} \sum_{i_{1}=1}^{\infty} \sum_{j_{1}=0}^{\infty} \sum_{k_{1}=0}^{\infty} \sum_{l_{1}=0}^{\infty} \sum_{m_{1}=0}^{\infty} i_{1} P_{i_{1} j_{1} k_{1} l_{1} m_{1}} \\
& -k_{t r p} \sum_{i=1}^{\infty} \sum_{j=0}^{\infty} \sum_{k=0}^{\infty} \sum_{l=0}^{\infty} \sum_{m=0}^{\infty} i P_{i j k l m} \sum_{i_{1}=1}^{\infty} \sum_{j_{1}=0}^{\infty} \sum_{k_{1}=0}^{\infty} \sum_{l_{1}=0}^{\infty} \sum_{m_{1}=0}^{\infty} k_{1} P_{i_{1} j_{1} k_{1} l_{1} m_{1}} \\
& +k_{t r p} \sum_{i=1}^{\infty} \sum_{j=0}^{\infty} \sum_{k=0}^{\infty} \sum_{l=0}^{\infty} \sum_{m=0}^{\infty}(k+1) P_{i j k+1 l m} \sum_{i_{1}=1}^{\infty} \sum_{j_{1}=0}^{\infty} \sum_{k_{1}=0}^{\infty} \sum_{l_{1}=0}^{\infty} \sum_{m_{1}=0}^{\infty} i_{1} P_{i_{1} j_{1} k_{1} l_{1} m_{1}} \\
& +k_{t r p} \sum_{i=1}^{\infty} \sum_{j=1}^{\infty} \sum_{k=1}^{\infty} \sum_{l=0}^{\infty} \sum_{m=0}^{\infty} i P_{i j-1 k-1 l m} \sum_{i_{1}=1}^{\infty} \sum_{j_{1}=0}^{\infty} \sum_{k_{1}=0}^{\infty} \sum_{l_{1}=0}^{\infty} \sum_{m_{1}=0}^{\infty} k_{1} P_{i_{1} j_{1} k_{1} l_{1} m_{1}}
\end{aligned}
$$




$$
\begin{aligned}
& -k_{t r p} \sum_{i=1}^{\infty} \sum_{j=0}^{\infty} \sum_{k=0}^{\infty} \sum_{l=0}^{\infty} \sum_{m=0}^{\infty} m P_{i j k l m} \sum_{i_{1}=1}^{\infty} \sum_{j_{1}=0}^{\infty} \sum_{k_{1}=0}^{\infty} \sum_{l_{1}=0}^{\infty} \sum_{m_{1}=0}^{\infty} i_{1} P_{i_{1} j_{1} k_{1} l_{1} m_{1}} \\
& -k_{t r p} \sum_{i=1}^{\infty} \sum_{j=0}^{\infty} \sum_{k=0}^{\infty} \sum_{l=0}^{\infty} \sum_{m=0}^{\infty} i P_{i j k l m} \sum_{i_{1}=1}^{\infty} \sum_{j_{1}=0}^{\infty} \sum_{k_{1}=0}^{\infty} \sum_{l_{1}=0}^{\infty} \sum_{m_{1}=0}^{\infty} m_{1} P_{i_{1} j_{1} k_{1} l_{1} m_{1}} \\
& +k_{t r p} \sum_{i=1}^{\infty} \sum_{j=0}^{\infty} \sum_{k=0}^{\infty} \sum_{l=0}^{\infty} \sum_{m=0}^{\infty}(m+1) P_{i j k l m+1} \sum_{i_{1}=1}^{\infty} \sum_{j_{1}=0}^{\infty} \sum_{k_{1}=0}^{\infty} \sum_{l_{1}=0}^{\infty} \sum_{m_{1}=0}^{\infty} i_{1} P_{i_{1} j_{1} k_{1} l_{1} m_{1}} \\
& +k_{\operatorname{trp}} \sum_{i=1}^{\infty} \sum_{j=1}^{\infty} \sum_{k=0}^{\infty} \sum_{l=0}^{\infty} \sum_{m=1}^{\infty} i P_{i j-1 k l m-1} \sum_{i_{1}=1}^{\infty} \sum_{j_{1}=0}^{\infty} \sum_{k_{1}=0}^{\infty} \sum_{l_{1}=0}^{\infty} \sum_{m_{1}=0}^{\infty} m_{1} P_{i_{1} j_{1} k_{1} l_{1} m_{1}} \\
& -k_{i n t r} \sum_{i=1}^{\infty} \sum_{j=0}^{\infty} \sum_{k=0}^{\infty} \sum_{l=0}^{\infty} \sum_{m=0}^{\infty} k P_{i j k l m} \\
& +k_{i n t r} \sum_{i=1}^{\infty} \sum_{j=1}^{\infty} \sum_{k=0}^{\infty} \sum_{l=0}^{\infty} \sum_{m=0}^{\infty} k P_{i j-1 k l m} \\
& -k_{r} \sum_{i=1}^{\infty} \sum_{j=0}^{\infty} \sum_{k=0}^{\infty} \sum_{l=0}^{\infty} \sum_{m=0}^{\infty} m P_{i j k l m} \\
& +k_{r} \sum_{i=1}^{\infty} \sum_{j=0}^{\infty} \sum_{k=1}^{\infty} \sum_{l=0}^{\infty} \sum_{m=0}^{\infty}(m+1) P_{i j k-1 l m+1} \\
& -k_{t c} \sum_{i=1}^{\infty} \sum_{j=0}^{\infty} \sum_{k=0}^{\infty} \sum_{l=0}^{\infty} \sum_{m=0}^{\infty} k P_{i j k l m} \sum_{i_{1}=1}^{\infty} \sum_{j_{1}=0}^{\infty} \sum_{k_{1}=0}^{\infty} \sum_{l_{1}=0}^{\infty} \sum_{m_{1}=0}^{\infty} k_{1} P_{i_{1} j_{1} k_{1} l_{1} m_{1}} \\
& +\frac{1}{2} k_{t c} \sum_{i=1}^{\infty} \sum_{j=0}^{\infty} \sum_{k=0}^{\infty} \sum_{l=0}^{\infty} \sum_{m=0}^{\infty} \sum_{i_{1}=1}^{i} \sum_{j_{1}=0}^{j} \sum_{k_{1}=0}^{k+2} \sum_{l_{1}=0}^{l} \sum_{m_{1}=0}^{m}\left[k_{1} P_{i_{1} j_{1} k_{1} l_{1} m_{1}}\left(k+2-k_{1}\right) P_{i-i_{1}, j-j_{1}, k+2-k_{1}, l-l_{1} m-m_{1}}\right] \\
& -k_{t c} \sum_{i=1}^{\infty} \sum_{j=0}^{\infty} \sum_{k=0}^{\infty} \sum_{l=0}^{\infty} \sum_{m=0}^{\infty} m P_{i j k l m} \sum_{i_{1}=1}^{\infty} \sum_{j_{1}=0}^{\infty} \sum_{k_{1}=0}^{\infty} \sum_{l_{1}=0}^{\infty} \sum_{m_{1}=0}^{\infty} m_{1} P_{i_{1} j_{1} k_{1} l_{1} m_{1}} \\
& +\frac{1}{2} k_{t c} \sum_{i=1}^{\infty} \sum_{j=0}^{\infty} \sum_{k=0}^{\infty} \sum_{l=0}^{\infty} \sum_{m=0}^{\infty} \sum_{i_{1}=1}^{i} \sum_{j_{1}=0}^{j} \sum_{k_{1}=0}^{k} \sum_{l_{1}=0}^{l} \sum_{m_{1}=0}^{m+2}\left[m_{1} P_{i_{1} j_{1} k_{1} l_{1} m_{1}}\left(m+2-m_{1}\right) P_{i-i_{1}, j-j_{1}, k-k_{1}, l-l_{1} m+2-m_{1}}\right] \\
& -k_{t c} \sum_{i=1}^{\infty} \sum_{j=0}^{\infty} \sum_{k=0}^{\infty} \sum_{l=0}^{\infty} \sum_{m=0}^{\infty} k P_{i j k l m} \sum_{i_{1}=1}^{\infty} \sum_{j_{1}=0}^{\infty} \sum_{k_{1}=0}^{\infty} \sum_{l_{1}=0}^{\infty} \sum_{m_{1}=0}^{\infty} m_{1} P_{i_{1} j_{1} k_{1} l_{1} m_{1}} \\
& -k_{t c} \sum_{i=1}^{\infty} \sum_{j=0}^{\infty} \sum_{k=0}^{\infty} \sum_{l=0}^{\infty} \sum_{m=0}^{\infty} m P_{i j k l m} \sum_{i_{1}=1}^{\infty} \sum_{j_{1}=0}^{\infty} \sum_{k_{1}=0}^{\infty} \sum_{l_{1}=0}^{\infty} \sum_{m_{1}=0}^{\infty} k_{1} P_{i_{1} j_{1} k_{1} l_{1} m_{1}} \\
& +\frac{1}{2} k_{t c} \sum_{i=1}^{\infty} \sum_{j=0}^{\infty} \sum_{k=0}^{\infty} \sum_{l=0}^{\infty} \sum_{m=0}^{\infty} \sum_{i_{1}=1}^{i} \sum_{j_{1}=0}^{j} \sum_{k_{1}=0}^{k+1} \sum_{l_{1}=0}^{l} \sum_{m_{1}=0}^{m+1}\left[k_{1} P_{i_{1} j_{1} k_{1} l_{1} m_{1}}\left(m+1-m_{1}\right) P_{i-i_{1}, j-j_{1}, k+1-k_{1}, l-l_{1} m+1-m_{1}}\right] \\
& +\frac{1}{2} k_{t c} \sum_{i=1}^{\infty} \sum_{j=0}^{\infty} \sum_{k=0}^{\infty} \sum_{l=0}^{\infty} \sum_{m=0}^{\infty} \sum_{i_{1}=1}^{i} \sum_{j_{1}=0}^{j} \sum_{k_{1}=0}^{k+1} \sum_{l_{1}=0}^{l} \sum_{m_{1}=0}^{m+1}\left[m_{1} P_{i_{1} j_{1} k_{1} l_{1} m_{1}}\left(k+1-k_{1}\right) P_{\left.i-i_{1}, j-j_{1}, k+1-k_{1}, l-l_{1} m+1-m_{1}\right]}\right.
\end{aligned}
$$




$$
\begin{aligned}
& \Rightarrow \frac{d \mu_{00000}}{d t}=k_{i} C_{M} C_{R}+k_{t m} C_{M} \sum_{i=1}^{\infty} \sum_{j=0}^{\infty} \sum_{k=0}^{\infty} \sum_{l=0}^{\infty} \sum_{m=0}^{\infty} k P_{i j k l m} \\
& -k_{p} C_{M} \sum_{i=1}^{\infty} \sum_{j=0}^{\infty} \sum_{k=0}^{\infty} \sum_{l=0}^{\infty} \sum_{m=0}^{\infty} k P_{i j k l m} \\
& +k_{p} C_{M} \sum_{i=1}^{\infty} \sum_{j=0}^{\infty} \sum_{k=0}^{\infty} \sum_{l=0}^{\infty} \sum_{m=0}^{\infty} k P_{i j k l m} \\
& -k_{p} C_{M} \sum_{i=1}^{\infty} \sum_{j=0}^{\infty} \sum_{k=0}^{\infty} \sum_{l=0}^{\infty} \sum_{m=0}^{\infty} m P_{i j k l m} \\
& +k_{p} C_{M} \sum_{i=1}^{\infty} \sum_{j=0}^{\infty} \sum_{k=0}^{\infty} \sum_{l=0}^{\infty} \sum_{m=0}^{\infty} m P_{i j k l m} \\
& -k_{t r t} C_{T} \sum_{i=1}^{\infty} \sum_{j=0}^{\infty} \sum_{k=0}^{\infty} \sum_{l=0}^{\infty} \sum_{m=0}^{\infty} k P_{i j k l m} \\
& +k_{t r t} C_{T} \sum_{i=1}^{\infty} \sum_{j=0}^{\infty} \sum_{k=0}^{\infty} \sum_{l=0}^{\infty} \sum_{m=0}^{\infty} k P_{i j k l m} \\
& -k_{t r t} \sum_{i=1}^{\infty} \sum_{j=0}^{\infty} \sum_{k=0}^{\infty} \sum_{l=0}^{\infty} \sum_{m=0}^{\infty} k P_{i j k l m} \sum_{i_{1}=1}^{\infty} \sum_{j_{1}=0}^{\infty} \sum_{k_{1}=0}^{\infty} \sum_{l_{1}=0}^{\infty} \sum_{m_{1}=0}^{\infty} l_{1} P_{i_{1} j_{1} k_{1} l_{1} m_{1}} \\
& -k_{t r t} \sum_{i=1}^{\infty} \sum_{j=0}^{\infty} \sum_{k=0}^{\infty} \sum_{l=0}^{\infty} \sum_{m=0}^{\infty} l P_{i j k l m} \sum_{i_{1}=1}^{\infty} \sum_{j_{1}=0}^{\infty} \sum_{k_{1}=0}^{\infty} \sum_{l_{1}=0}^{\infty} \sum_{m_{1}=0}^{\infty} k_{1} P_{i_{1} j_{1} k_{1} l_{1} m_{1}} \\
& +k_{t r t} \sum_{i=1}^{\infty} \sum_{j=0}^{\infty} \sum_{k=0}^{\infty} \sum_{l=0}^{\infty} \sum_{m=0}^{\infty} k P_{i j k l m} \sum_{i_{1}=1}^{\infty} \sum_{j_{1}=0}^{\infty} \sum_{k_{1}=0}^{\infty} \sum_{l_{1}=0}^{\infty} \sum_{m_{1}=0}^{\infty} l_{1} P_{i_{1} j_{1} k_{1} l_{1} m_{1}} \\
& +k_{t r t} \sum_{i=1}^{\infty} \sum_{j=0}^{\infty} \sum_{k=0}^{\infty} \sum_{l=0}^{\infty} \sum_{m=0}^{\infty} l P_{i j k l m} \sum_{i_{1}=1}^{\infty} \sum_{j_{1}=0}^{\infty} \sum_{k_{1}=0}^{\infty} \sum_{l_{1}=0}^{\infty} \sum_{m_{1}=0}^{\infty} k_{1} P_{i_{1} j_{1} k_{1} l_{1} m_{1}} \\
& -k_{t r p} \sum_{i=1}^{\infty} \sum_{j=0}^{\infty} \sum_{k=0}^{\infty} \sum_{l=0}^{\infty} \sum_{m=0}^{\infty} k P_{i j k l m} \sum_{i_{1}=1}^{\infty} \sum_{j_{1}=0}^{\infty} \sum_{k_{1}=0}^{\infty} \sum_{l_{1}=0}^{\infty} \sum_{m_{1}=0}^{\infty} i_{1} P_{i_{1} j_{1} k_{1} l_{1} m_{1}} \\
& -k_{t r p} \sum_{i=1}^{\infty} \sum_{j=0}^{\infty} \sum_{k=0}^{\infty} \sum_{l=0}^{\infty} \sum_{m=0}^{\infty} i P_{i j k l m} \sum_{i_{1}=1}^{\infty} \sum_{j_{1}=0}^{\infty} \sum_{k_{1}=0}^{\infty} \sum_{l_{1}=0}^{\infty} \sum_{m_{1}=0}^{\infty} k_{1} P_{i_{1} j_{1} k_{1} l_{1} m_{1}} \\
& +k_{t r p} \sum_{i=1}^{\infty} \sum_{j=0}^{\infty} \sum_{k=0}^{\infty} \sum_{l=0}^{\infty} \sum_{m=0}^{\infty} k P_{i j k l m} \sum_{i_{1}=1}^{\infty} \sum_{j_{1}=0}^{\infty} \sum_{k_{1}=0}^{\infty} \sum_{l_{1}=0}^{\infty} \sum_{m_{1}=0}^{\infty} i_{1} P_{i_{1} j_{1} k_{1} l_{1} m_{1}} \\
& +k_{\text {trp }} \sum_{i=1}^{\infty} \sum_{j=0}^{\infty} \sum_{k=0}^{\infty} \sum_{l=0}^{\infty} \sum_{m=0}^{\infty} i P_{i j k l m} \sum_{i_{1}=1}^{\infty} \sum_{j_{1}=0}^{\infty} \sum_{k_{1}=0}^{\infty} \sum_{l_{1}=0}^{\infty} \sum_{m_{1}=0}^{\infty} k_{1} P_{i_{1} j_{1} k_{1} l_{1} m_{1}}
\end{aligned}
$$


$-k_{t r p} \sum_{i=1}^{\infty} \sum_{j=0}^{\infty} \sum_{k=0}^{\infty} \sum_{l=0}^{\infty} \sum_{m=0}^{\infty} m P_{i j k l m} \sum_{i_{1}=1}^{\infty} \sum_{j_{1}=0}^{\infty} \sum_{k_{1}=0}^{\infty} \sum_{l_{1}=0}^{\infty} \sum_{m_{1}=0}^{\infty} i_{1} P_{i_{1} j_{1} k_{1} l_{1} m_{1}}$

$-k_{\text {trp }} \sum_{i=1}^{\infty} \sum_{j=0}^{\infty} \sum_{k=0}^{\infty} \sum_{l=0}^{\infty} \sum_{m=0}^{\infty} i P_{i j k l m} \sum_{i_{1}=1}^{\infty} \sum_{j_{1}=0}^{\infty} \sum_{k_{1}=0}^{\infty} \sum_{l_{1}=0}^{\infty} \sum_{m_{1}=0}^{\infty} m_{1} P_{i_{1} j_{1} k_{1} l_{1} m_{1}}$

$+k_{\text {trp }} \sum_{i=1}^{\infty} \sum_{j=0}^{\infty} \sum_{k=0}^{\infty} \sum_{l=0}^{\infty} \sum_{m=0}^{\infty} m P_{i j k l m} \sum_{i_{1}=1}^{\infty} \sum_{j_{1}=0}^{\infty} \sum_{k_{1}=0}^{\infty} \sum_{l_{1}=0}^{\infty} \sum_{m_{1}=0}^{\infty} i_{1} P_{i_{1} j_{1} k_{1} l_{1} m_{1}}$

$+k_{t r p} \sum_{i=1}^{\infty} \sum_{j=0}^{\infty} \sum_{k=0}^{\infty} \sum_{l=0}^{\infty} \sum_{m=0}^{\infty} i P_{i j k l m} \sum_{i_{1}=1}^{\infty} \sum_{j_{1}=0}^{\infty} \sum_{k_{1}=0}^{\infty} \sum_{l_{1}=0}^{\infty} \sum_{m_{1}=0}^{\infty} m_{1} P_{i_{1} j_{1} k_{1} l_{1} m_{1}}$

$-k_{i n t r} \sum_{i=1}^{\infty} \sum_{j=0}^{\infty} \sum_{k=0}^{\infty} \sum_{l=0}^{\infty} \sum_{m=0}^{\infty} k P_{i j k l m}$

$+k_{\text {intr }} \sum_{i=1}^{\infty} \sum_{j=0}^{\infty} \sum_{k=0}^{\infty} \sum_{l=0}^{\infty} \sum_{m=0}^{\infty} k P_{i j k l m}$

$-k_{r} \sum_{i=1}^{\infty} \sum_{j=0}^{\infty} \sum_{k=0}^{\infty} \sum_{l=0}^{\infty} \sum_{m=0}^{\infty} m P_{i j k l m}$

$+k_{r} \sum_{i=1}^{\infty} \sum_{j=0}^{\infty} \sum_{k=0}^{\infty} \sum_{l=0}^{\infty} \sum_{m=0}^{\infty} m P_{i j k l m}$

$-k_{t c} \sum_{i=1}^{\infty} \sum_{j=0}^{\infty} \sum_{k=0}^{\infty} \sum_{l=0}^{\infty} \sum_{m=0}^{\infty} k P_{i j k l m} \sum_{i_{1}=1}^{\infty} \sum_{j_{1}=0}^{\infty} \sum_{k_{1}=0}^{\infty} \sum_{l_{1}=0}^{\infty} \sum_{m_{1}=0}^{\infty} k_{1} P_{i_{1} j_{1} k_{1} l_{1} m_{1}}$

$+\frac{1}{2} k_{t c} \sum_{i=1}^{\infty} \sum_{j=0}^{\infty} \sum_{k=0}^{\infty} \sum_{l=0}^{\infty} \sum_{m=0}^{\infty} k P_{i j k l m} \sum_{i_{1}=1}^{\infty} \sum_{j_{1}=0}^{\infty} \sum_{k_{1}=0}^{\infty} \sum_{l_{1}=0}^{\infty} \sum_{m_{1}=0}^{\infty} k_{1} P_{i_{1} j_{1} k_{1} l_{1} m_{1}}$

$-k_{t c} \sum_{i=1}^{\infty} \sum_{j=0}^{\infty} \sum_{k=0}^{\infty} \sum_{l=0}^{\infty} \sum_{m=0}^{\infty} m P_{i j k l m} \sum_{i_{1}=1}^{\infty} \sum_{j_{1}=0}^{\infty} \sum_{k_{1}=0}^{\infty} \sum_{l_{1}=0}^{\infty} \sum_{m_{1}=0}^{\infty} m_{1} P_{i_{1} j_{1} k_{1} l_{1} m_{1}}$

$+\frac{1}{2} k_{t c} \sum_{i=1}^{\infty} \sum_{j=0}^{\infty} \sum_{k=0}^{\infty} \sum_{l=0}^{\infty} \sum_{m=0}^{\infty} m P_{i j k l m} \sum_{i_{1}=1}^{\infty} \sum_{j_{1}=0}^{\infty} \sum_{k_{1}=0}^{\infty} \sum_{l_{1}=0}^{\infty} \sum_{m_{1}=0}^{\infty} m_{1} P_{i_{1} j_{1} k_{1} l_{1} m_{1}}$

$-k_{t c} \sum_{i=1}^{\infty} \sum_{j=0}^{\infty} \sum_{k=0}^{\infty} \sum_{l=0}^{\infty} \sum_{m=0}^{\infty} k P_{i j k l m} \sum_{i_{1}=1}^{\infty} \sum_{j_{1}=0}^{\infty} \sum_{k_{1}=0}^{\infty} \sum_{l_{1}=0}^{\infty} \sum_{m_{1}=0}^{\infty} m_{1} P_{i_{1} j_{1} k_{1} l_{1} m_{1}}$

$-k_{t c} \sum_{i=1}^{\infty} \sum_{j=0}^{\infty} \sum_{k=0}^{\infty} \sum_{l=0}^{\infty} \sum_{m=0}^{\infty} m P_{i j k l m} \sum_{i_{1}=1}^{\infty} \sum_{j_{1}=0}^{\infty} \sum_{k_{1}=0}^{\infty} \sum_{l_{1}=0}^{\infty} \sum_{m_{1}=0}^{\infty} k_{1} P_{i_{1} j_{1} k_{1} l_{1} m_{1}}$

$+\frac{1}{2} k_{t c} \sum_{i=1}^{\infty} \sum_{j=0}^{\infty} \sum_{k=0}^{\infty} \sum_{l=0}^{\infty} \sum_{m=0}^{\infty} k P_{i j k l m} \sum_{i_{1}=1}^{\infty} \sum_{j_{1}=0}^{\infty} \sum_{k_{1}=0}^{\infty} \sum_{l_{1}=0}^{\infty} \sum_{m_{1}=0}^{\infty} m_{1} P_{i_{1} j_{1} k_{1} l_{1} m_{1}}$

$+\frac{1}{2} k_{t c} \sum_{i=1}^{\infty} \sum_{j=0}^{\infty} \sum_{k=0}^{\infty} \sum_{l=0}^{\infty} \sum_{m=0}^{\infty} m P_{i j k l m} \sum_{i_{1}=1}^{\infty} \sum_{j_{1}=0}^{\infty} \sum_{k_{1}=0}^{\infty} \sum_{l_{1}=0}^{\infty} \sum_{m_{1}=0}^{\infty} k_{1} P_{i_{1} j_{1} k_{1} l_{1} m_{1}}$

because $\sum_{n=0}^{\infty} \sum_{m=0}^{n} a_{m} b_{n-m}=\sum_{n=0}^{\infty} a_{n} \sum_{m=0}^{\infty} b_{m}$ 


$$
\begin{aligned}
& \Rightarrow \frac{d \mu_{00000}}{d t}=k_{i} C_{M} C_{R}-\frac{1}{2} k_{t c} \sum_{i=1}^{\infty} \sum_{j=0}^{\infty} \sum_{k=0}^{\infty} \sum_{l=0}^{\infty} \sum_{m=0}^{\infty} k P_{i j k l m} \sum_{i_{1}=1}^{\infty} \sum_{j_{1}=0}^{\infty} \sum_{k_{1}=0}^{\infty} \sum_{l_{1}=0}^{\infty} \sum_{m_{1}=0}^{\infty} k_{1} P_{i_{1} j_{1} k_{1} l_{1} m_{1}} \\
& \quad-\frac{1}{2} k_{t c} \sum_{i=1}^{\infty} \sum_{j=0}^{\infty} \sum_{k=0}^{\infty} \sum_{l=0}^{\infty} \sum_{m=0}^{\infty} m P_{i j k l m} \sum_{i_{1}=1}^{\infty} \sum_{j_{1}=0}^{\infty} \sum_{k_{1}=0}^{\infty} \sum_{l_{1}=0}^{\infty} \sum_{m_{1}=0}^{\infty} m_{1} P_{i_{1} j_{1} k_{1} l_{1} m_{1}} \\
& \quad-k_{t c} \sum_{i=1}^{\infty} \sum_{j=0}^{\infty} \sum_{k=0}^{\infty} \sum_{l=0}^{\infty} \sum_{m=0}^{\infty} k P_{i j k l m} \sum_{i_{1}=1}^{\infty} \sum_{j_{1}=0}^{\infty} \sum_{k_{1}=0}^{\infty} \sum_{l_{1}=0}^{\infty} \sum_{m_{1}=0}^{\infty} m_{1} P_{i_{1} j_{1} k_{1} l_{1} m_{1}} \\
& \Rightarrow \frac{d \mu_{00000}}{d t}=k_{i} C_{M} C_{R}-\frac{1}{2} k_{t c}\left(\mu_{00100}\right)^{2}-\frac{1}{2} k_{t c}\left(\mu_{00001}\right)^{2}-k_{t c} \mu_{00100} \mu_{00001}
\end{aligned}
$$




\subsection{First moments}

3.2.1 First moment w.r.t. the number of repeat units

$$
\begin{aligned}
& \frac{d \mu_{10000}}{d t}=\sum_{i=1}^{\infty} \sum_{j=0}^{\infty} \sum_{k=0}^{\infty} \sum_{l=0}^{\infty} \sum_{m=0}^{\infty} i \frac{d P_{i j k l m}}{d t} \\
& =k_{i} C_{M} C_{R} \\
& -k_{p} C_{M} \sum_{i=1}^{\infty} \sum_{j=0}^{\infty} \sum_{k=0}^{\infty} \sum_{l=0}^{\infty} \sum_{m=0}^{\infty} i k P_{i j k l m} \\
& +k_{p} C_{M} \sum_{i=2}^{\infty} \sum_{j=0}^{\infty} \sum_{k=0}^{\infty} \sum_{l=0}^{\infty} \sum_{m=0}^{\infty} i k P_{i-1 j k l m} \\
& -k_{p} C_{M} \sum_{i=1}^{\infty} \sum_{j=0}^{\infty} \sum_{k=0}^{\infty} \sum_{l=0}^{\infty} \sum_{m=0}^{\infty} i m P_{i j k l m} \\
& +k_{p} C_{M} \sum_{i=2}^{\infty} \sum_{j=0}^{\infty} \sum_{k=0}^{\infty} \sum_{l=0}^{\infty} \sum_{m=0}^{\infty} i m P_{i-1 j k l m} \\
& -k_{t r t} C_{T} \sum_{i=1}^{\infty} \sum_{j=0}^{\infty} \sum_{k=0}^{\infty} \sum_{l=0}^{\infty} \sum_{m=0}^{\infty} i k P_{i j k l m} \\
& +k_{t r t} C_{T} \sum_{i=1}^{\infty} \sum_{j=0}^{\infty} \sum_{k=0}^{\infty} \sum_{l=1}^{\infty} \sum_{m=0}^{\infty} i(k+1) P_{i j k+1 l-1 m} \\
& -k_{t r t} \sum_{i=1}^{\infty} \sum_{j=0}^{\infty} \sum_{k=0}^{\infty} \sum_{l=0}^{\infty} \sum_{m=0}^{\infty} i k P_{i j k l m} \sum_{i_{1}=1}^{\infty} \sum_{j_{1}=0}^{\infty} \sum_{k_{1}=0}^{\infty} \sum_{l_{1}=0}^{\infty} \sum_{m_{1}=0}^{\infty} l_{1} P_{i_{1} j_{1} k_{1} l_{1} m_{1}} \\
& -k_{t r t} \sum_{i=1}^{\infty} \sum_{j=0}^{\infty} \sum_{k=0}^{\infty} \sum_{l=0}^{\infty} \sum_{m=0}^{\infty} i l P_{i j k l m} \sum_{i_{1}=1}^{\infty} \sum_{j_{1}=0}^{\infty} \sum_{k_{1}=0}^{\infty} \sum_{l_{1}=0}^{\infty} \sum_{m_{1}=0}^{\infty} k_{1} P_{i_{1} j_{1} k_{1} l_{1} m_{1}} \\
& +k_{t r t} \sum_{i=1}^{\infty} \sum_{j=0}^{\infty} \sum_{k=0}^{\infty} \sum_{l=1}^{\infty} \sum_{m=0}^{\infty} i(k+1) P_{i j k+1 l-1 m} \sum_{i_{1}=1}^{\infty} \sum_{j_{1}=0}^{\infty} \sum_{k_{1}=0}^{\infty} \sum_{l_{1}=0}^{\infty} \sum_{m_{1}=0}^{\infty} l_{1} P_{i_{1} j_{1} k_{1} l_{1} m_{1}} \\
& +k_{t r t} \sum_{i=1}^{\infty} \sum_{j=0}^{\infty} \sum_{k=0}^{\infty} \sum_{l=0}^{\infty} \sum_{m=1}^{\infty} i(l+1) P_{i j k l+1 m-1} \sum_{i_{1}=1}^{\infty} \sum_{j_{1}=0}^{\infty} \sum_{k_{1}=0}^{\infty} \sum_{l_{1}=0}^{\infty} \sum_{m_{1}=0}^{\infty} k_{1} P_{i_{1} j_{1} k_{1} l_{1} m_{1}} \\
& -k_{\operatorname{trp}} \sum_{i=1}^{\infty} \sum_{j=0}^{\infty} \sum_{k=0}^{\infty} \sum_{l=0}^{\infty} \sum_{m=0}^{\infty} i k P_{i j k l m} \sum_{i_{1}=1}^{\infty} \sum_{j_{1}=0}^{\infty} \sum_{k_{1}=0}^{\infty} \sum_{l_{1}=0}^{\infty} \sum_{m_{1}=0}^{\infty} i_{1} P_{i_{1} j_{1} k_{1} l_{1} m_{1}} \\
& -k_{\operatorname{trp}} \sum_{i=1}^{\infty} \sum_{j=0}^{\infty} \sum_{k=0}^{\infty} \sum_{l=0}^{\infty} \sum_{m=0}^{\infty} i^{2} P_{i j k l m} \sum_{i_{1}=1}^{\infty} \sum_{j_{1}=0}^{\infty} \sum_{k_{1}=0}^{\infty} \sum_{l_{1}=0}^{\infty} \sum_{m_{1}=0}^{\infty} k_{1} P_{i_{1} j_{1} k_{1} l_{1} m_{1}} \\
& +k_{t r p} \sum_{i=1}^{\infty} \sum_{j=0}^{\infty} \sum_{k=0}^{\infty} \sum_{l=0}^{\infty} \sum_{m=0}^{\infty} i(k+1) P_{i j k+1 l m} \sum_{i_{1}=1}^{\infty} \sum_{j_{1}=0}^{\infty} \sum_{k_{1}=0}^{\infty} \sum_{l_{1}=0}^{\infty} \sum_{m_{1}=0}^{\infty} i_{1} P_{i_{1} j_{1} k_{1} l_{1} m_{1}} \\
& +k_{\operatorname{trp}} \sum_{i=1}^{\infty} \sum_{j=1}^{\infty} \sum_{k=1}^{\infty} \sum_{l=0}^{\infty} \sum_{m=0}^{\infty} i^{2} P_{i j-1 k-1 l m} \sum_{i_{1}=1}^{\infty} \sum_{j_{1}=0}^{\infty} \sum_{k_{1}=0}^{\infty} \sum_{l_{1}=0}^{\infty} \sum_{m_{1}=0}^{\infty} k_{1} P_{i_{1} j_{1} k_{1} l_{1} m_{1}}
\end{aligned}
$$




$$
\begin{aligned}
& -k_{t r p} \sum_{i=1}^{\infty} \sum_{j=0}^{\infty} \sum_{k=0}^{\infty} \sum_{l=0}^{\infty} \sum_{m=0}^{\infty} i m P_{i j k l m} \sum_{i_{1}=1}^{\infty} \sum_{j_{1}=0}^{\infty} \sum_{k_{1}=0}^{\infty} \sum_{l_{1}=0}^{\infty} \sum_{m_{1}=0}^{\infty} i_{1} P_{i_{1} j_{1} k_{1} l_{1} m_{1}} \\
& -k_{t r p} \sum_{i=1}^{\infty} \sum_{j=0}^{\infty} \sum_{k=0}^{\infty} \sum_{l=0}^{\infty} \sum_{m=0}^{\infty} i^{2} P_{i j k l m} \sum_{i_{1}=1}^{\infty} \sum_{j_{1}=0}^{\infty} \sum_{k_{1}=0}^{\infty} \sum_{l_{1}=0}^{\infty} \sum_{m_{1}=0}^{\infty} m_{1} P_{i_{1} j_{1} k_{1} l_{1} m_{1}} \\
& +k_{\text {trp }} \sum_{i=1}^{\infty} \sum_{j=0}^{\infty} \sum_{k=0}^{\infty} \sum_{l=0}^{\infty} \sum_{m=0}^{\infty} i(m+1) P_{i j k l m+1} \sum_{i_{1}=1}^{\infty} \sum_{j_{1}=0}^{\infty} \sum_{k_{1}=0}^{\infty} \sum_{l_{1}=0}^{\infty} \sum_{m_{1}=0}^{\infty} i_{1} P_{i_{1} j_{1} k_{1} l_{1} m_{1}} \\
& +k_{t r p} \sum_{i=1}^{\infty} \sum_{j=1}^{\infty} \sum_{k=0}^{\infty} \sum_{l=0}^{\infty} \sum_{m=1}^{\infty} i^{2} P_{i j-1 k l m-1} \sum_{i_{1}=1}^{\infty} \sum_{j_{1}=0}^{\infty} \sum_{k_{1}=0}^{\infty} \sum_{l_{1}=0}^{\infty} \sum_{m_{1}=0}^{\infty} m_{1} P_{i_{1} j_{1} k_{1} l_{1} m_{1}} \\
& -k_{i n t r} \sum_{i=1}^{\infty} \sum_{j=0}^{\infty} \sum_{k=0}^{\infty} \sum_{l=0}^{\infty} \sum_{m=0}^{\infty} i k P_{i j k l m} \\
& +k_{i n t r} \sum_{i=1}^{\infty} \sum_{j=1}^{\infty} \sum_{k=0}^{\infty} \sum_{l=0}^{\infty} \sum_{m=0}^{\infty} i k P_{i j-1 k l m} \\
& -k_{r} \sum_{i=1}^{\infty} \sum_{j=0}^{\infty} \sum_{k=0}^{\infty} \sum_{l=0}^{\infty} \sum_{m=0}^{\infty} i m P_{i j k l m} \\
& +k_{r} \sum_{i=1}^{\infty} \sum_{j=0}^{\infty} \sum_{k=1}^{\infty} \sum_{l=0}^{\infty} \sum_{m=0}^{\infty} i(m+1) P_{i j k-1 l m+1} \\
& -k_{t c} \sum_{i=1}^{\infty} \sum_{j=0}^{\infty} \sum_{k=0}^{\infty} \sum_{l=0}^{\infty} \sum_{m=0}^{\infty} i k P_{i j k l m} \sum_{i_{1}=1}^{\infty} \sum_{j_{1}=0}^{\infty} \sum_{k_{1}=0}^{\infty} \sum_{l_{1}=0}^{\infty} \sum_{m_{1}=0}^{\infty} k_{1} P_{i_{1} j_{1} k_{1} l_{1} m_{1}} \\
& +\frac{1}{2} k_{t c} \sum_{i=1}^{\infty} \sum_{j=0}^{\infty} \sum_{k=0}^{\infty} \sum_{l=0}^{\infty} \sum_{m=0}^{\infty} \sum_{i_{1}=1}^{i} \sum_{j_{1}=0}^{j} \sum_{k_{1}=0}^{k+2} \sum_{l_{1}=0}^{l} \sum_{m_{1}=0}^{m}\left[i k_{1} P_{i_{1} j_{1} k_{1} l_{1} m_{1}}\left(k+2-k_{1}\right) P_{i-i_{1}, j-j_{1}, k+2-k_{1}, l-l_{1} m-m_{1}}\right] \\
& -k_{t c} \sum_{i=1}^{\infty} \sum_{j=0}^{\infty} \sum_{k=0}^{\infty} \sum_{l=0}^{\infty} \sum_{m=0}^{\infty} i m P_{i j k l m} \sum_{i_{1}=1}^{\infty} \sum_{j_{1}=0}^{\infty} \sum_{k_{1}=0}^{\infty} \sum_{l_{1}=0}^{\infty} \sum_{m_{1}=0}^{\infty} m_{1} P_{i_{1} j_{1} k_{1} l_{1} m_{1}} \\
& +\frac{1}{2} k_{t c} \sum_{i=1}^{\infty} \sum_{j=0}^{\infty} \sum_{k=0}^{\infty} \sum_{l=0}^{\infty} \sum_{m=0}^{\infty} \sum_{i_{1}=1}^{i} \sum_{j_{1}=0}^{j} \sum_{k_{1}=0}^{k} \sum_{l_{1}=0}^{l} \sum_{m_{1}=0}^{m+2}\left[i m_{1} P_{i_{1} j_{1} k_{1} l_{1} m_{1}}\left(m+2-m_{1}\right) P_{i-i_{1}, j-j_{1}, k-k_{1}, l-l_{1} m+2-m_{1}}\right] \\
& -k_{t c} \sum_{i=1}^{\infty} \sum_{j=0}^{\infty} \sum_{k=0}^{\infty} \sum_{l=0}^{\infty} \sum_{m=0}^{\infty} i k P_{i j k l m} \sum_{i_{1}=1}^{\infty} \sum_{j_{1}=0}^{\infty} \sum_{k_{1}=0}^{\infty} \sum_{l_{1}=0}^{\infty} \sum_{m_{1}=0}^{\infty} m_{1} P_{i_{1} j_{1} k_{1} l_{1} m_{1}} \\
& -k_{t c} \sum_{i=1}^{\infty} \sum_{j=0}^{\infty} \sum_{k=0}^{\infty} \sum_{l=0}^{\infty} \sum_{m=0}^{\infty} i m P_{i j k l m} \sum_{i_{1}=1}^{\infty} \sum_{j_{1}=0}^{\infty} \sum_{k_{1}=0}^{\infty} \sum_{l_{1}=0}^{\infty} \sum_{m_{1}=0}^{\infty} k_{1} P_{i_{1} j_{1} k_{1} l_{1} m_{1}} \\
& +\frac{1}{2} k_{t c} \sum_{i=1}^{\infty} \sum_{j=0}^{\infty} \sum_{k=0}^{\infty} \sum_{l=0}^{\infty} \sum_{m=0}^{\infty} \sum_{i_{1}=1}^{i} \sum_{j_{1}=0}^{j} \sum_{k_{1}=0}^{k+1} \sum_{l_{1}=0}^{l} \sum_{m_{1}=0}^{m+1}\left[i k_{1} P_{i_{1} j_{1} k_{1} l_{1} m_{1}}\left(m+1-m_{1}\right) P_{i-i_{1}, j-j_{1}, k+1-k_{1}, l-l_{1} m+1-m_{1}}\right] \\
& +\frac{1}{2} k_{t c} \sum_{i=1}^{\infty} \sum_{j=0}^{\infty} \sum_{k=0}^{\infty} \sum_{l=0}^{\infty} \sum_{m=0}^{\infty} \sum_{i_{1}=1}^{i} \sum_{j_{1}=0}^{j} \sum_{k_{1}=0}^{k+1} \sum_{l_{1}=0}^{l} \sum_{m_{1}=0}^{m+1}\left[i m_{1} P_{i_{1} j_{1} k_{1} l_{1} m_{1}}\left(k+1-k_{1}\right) P_{i-i_{1}, j-j_{1}, k+1-k_{1}, l-l_{1} m+1-m_{1}}\right]
\end{aligned}
$$

Now

$$
(a+b)^{k}=\sum_{j=0}^{k}\left(\begin{array}{l}
k \\
j
\end{array}\right) a^{j} b^{k-j}
$$


where

$$
\left(\begin{array}{l}
k \\
j
\end{array}\right)=\frac{k !}{j !(k-j) !}
$$

Also,

$$
\sum_{n=0}^{\infty} \sum_{m=0}^{n} a_{m} b_{n-m}=\sum_{n=0}^{\infty} a_{n} \sum_{m=0}^{\infty} b_{m}
$$

Therefore

$$
\begin{aligned}
\sum_{i=1}^{\infty} \sum_{i_{1}=1}^{i} & \sum_{k=0}^{\infty} \sum_{k_{1}=0}^{k+2} i k_{1} P_{i_{1}, k_{1}}\left(k+2-k_{1}\right) P_{i-i_{1}, k+2-k_{1}} \\
& =\sum_{i=1}^{\infty} \sum_{i_{1}=1}^{i} \sum_{k=0}^{\infty} \sum_{k_{1}=0}^{k+2}\left[i_{1}+\left(i-i_{1}\right)\right] k_{1} P_{i_{1}, k_{1}}\left(k+2-k_{1}\right) P_{i-i_{1}, k+2-k_{1}} \\
& =\sum_{a=0}^{1}\left(\begin{array}{l}
1 \\
a
\end{array}\right) \sum_{i=1}^{\infty} \sum_{i_{1}=1}^{i} \sum_{k=0}^{\infty} \sum_{k_{1}=0}^{k+2} i_{1}^{a} k_{1} P_{i_{1}, k_{1}}\left(i-i_{1}\right)^{1-a}\left(k+2-k_{1}\right) P_{i-i_{1}, k+2-k_{1}} \\
& =\sum_{a=0}^{1}\left(\begin{array}{l}
1 \\
a
\end{array}\right) \sum_{i=1}^{\infty} \sum_{k=0}^{\infty} i^{a} k P_{i, k} \sum_{i_{1}=1}^{\infty} \sum_{k_{1}=0}^{\infty} i_{1}^{1-a} k_{1} P_{i_{1}, k_{1}} \\
& =\sum_{a=0}^{1}\left(\begin{array}{l}
1 \\
a
\end{array}\right) \mu_{a, 1} \mu_{1-a, 1} \\
= & \left(\frac{1 !}{0 ! 1 !} \mu_{01} \mu_{11}\right)+\left(\frac{1 !}{1 ! 0 !} \mu_{11} \mu_{01}\right) \\
= & 2 \mu_{01} \mu_{11}
\end{aligned}
$$

Similarly,

$$
\begin{aligned}
\sum_{i=1}^{\infty} \sum_{i_{1}=1}^{i} \sum_{k=0}^{\infty} \sum_{k_{1}=0}^{k+1} \sum_{m=1}^{\infty} \sum_{m_{1}=1}^{m+1} i k_{1} P_{i_{1}, k_{1}, m_{1}}\left(m+1-m_{1}\right) P_{i-i_{1}, k+1-k_{1}, m+1-m_{1}} \\
\sum_{i=1}^{\infty} \sum_{i_{1}=1}^{i} \sum_{k=0}^{\infty} \sum_{k_{1}=0}^{k+1} \sum_{m=1}^{\infty} \sum_{m_{1}=1}^{m+1}\left[i_{1}+\left(i-i_{1}\right)\right] k_{1} P_{i_{1}, k_{1}, m_{1}}\left(m+1-m_{1}\right) P_{i-i_{1}, k+1-k_{1}, m+1-m_{1}} \\
=\sum_{a=0}^{1}\left(\begin{array}{l}
1 \\
a
\end{array}\right) \sum_{i=1}^{\infty} \sum_{i_{1}=1}^{i} \sum_{k=0}^{\infty} \sum_{k_{1}=0}^{k+1} \sum_{m=1}^{\infty} \sum_{m_{1}=1}^{m+1} i_{1}^{a} k_{1} P_{i_{1}, k_{1}, m_{1}}\left(i-i_{1}\right)^{1-a}\left(m+1-m_{1}\right) P_{i-i_{1}, k+1-k_{1}, m+1-m_{1}} \\
=\sum_{a=0}^{1}\left(\begin{array}{l}
1 \\
a
\end{array}\right) \sum_{i=1}^{\infty} \sum_{k=0}^{\infty} \sum_{m=0}^{\infty} i^{a} k P_{i, k, m} \sum_{i_{1}=1}^{\infty} \sum_{k_{1}=0}^{\infty} \sum_{m_{1}=0}^{\infty} i_{1}^{1-a} m_{1} P_{i_{1}, k_{1}, m_{1}} \\
=\sum_{a=0}^{1}\left(\begin{array}{l}
1 \\
a
\end{array}\right) \mu_{a, 1,0} \mu_{1-a, 0,1} \\
=\left(\frac{1 !}{0 ! 1 !} \mu_{010} \mu_{101}\right)+\left(\frac{1 !}{1 ! 0 !} \mu_{110} \mu_{001}\right) \\
=\mu_{010} \mu_{101}+\mu_{110} \mu_{001}
\end{aligned}
$$




$$
\begin{aligned}
& \Rightarrow \frac{d \mu_{10000}}{d t}=k_{i} C_{M} C_{R}+k_{t m} C_{M} \sum_{i=1}^{\infty} \sum_{j=0}^{\infty} \sum_{k=0}^{\infty} \sum_{l=0}^{\infty} \sum_{m=0}^{\infty} i k P_{i j k l m} \\
& -k_{t m} C_{M} \sum_{i=1}^{\infty} \sum_{j=0}^{\infty} \sum_{k=0}^{\infty} \sum_{l=0}^{\infty} \sum_{m=0}^{\infty} i k P_{i j k l m} \\
& +k_{t m} C_{M} \sum_{i=1}^{\infty} \sum_{j=0}^{\infty} \sum_{k=0}^{\infty} \sum_{l=0}^{\infty} \sum_{m=0}^{\infty} i k P_{i j k l m} \\
& -k_{p} C_{M} \sum_{i=1}^{\infty} \sum_{j=0}^{\infty} \sum_{k=0}^{\infty} \sum_{l=0}^{\infty} \sum_{m=0}^{\infty} i k P_{i j k l m} \\
& +k_{p} C_{M} \sum_{i=1}^{\infty} \sum_{j=0}^{\infty} \sum_{k=0}^{\infty} \sum_{l=0}^{\infty} \sum_{m=0}^{\infty}(i+1) k P_{i j k l m} \\
& -k_{p} C_{M} \sum_{i=1}^{\infty} \sum_{j=0}^{\infty} \sum_{k=0}^{\infty} \sum_{l=0}^{\infty} \sum_{m=0}^{\infty} i m P_{i j k l m} \\
& +k_{p} C_{M} \sum_{i=1}^{\infty} \sum_{j=0}^{\infty} \sum_{k=0}^{\infty} \sum_{l=0}^{\infty} \sum_{m=0}^{\infty}(i+1) m P_{i j k l m} \\
& -k_{t r t} C_{T} \sum_{i=1}^{\infty} \sum_{j=0}^{\infty} \sum_{k=0}^{\infty} \sum_{l=0}^{\infty} \sum_{m=0}^{\infty} i k P_{i j k l m} \\
& +k_{t r t} C_{T} \sum_{i=1}^{\infty} \sum_{j=0}^{\infty} \sum_{k=0}^{\infty} \sum_{l=0}^{\infty} \sum_{m=0}^{\infty} i k P_{i j k l m} \\
& -k_{t r t} \sum_{i=1}^{\infty} \sum_{j=0}^{\infty} \sum_{k=0}^{\infty} \sum_{l=0}^{\infty} \sum_{m=0}^{\infty} i k P_{i j k l m} \sum_{i_{1}=1}^{\infty} \sum_{j_{1}=0}^{\infty} \sum_{k_{1}=0}^{\infty} \sum_{l_{1}=0}^{\infty} \sum_{m_{1}=0}^{\infty} l_{1} P_{i_{1} j_{1} k_{1} l_{1} m_{1}} \\
& -k_{t r t} \sum_{i=1}^{\infty} \sum_{j=0}^{\infty} \sum_{k=0}^{\infty} \sum_{l=0}^{\infty} \sum_{m=0}^{\infty} i l P_{i j k l m} \sum_{i_{1}=1}^{\infty} \sum_{j_{1}=0}^{\infty} \sum_{k_{1}=0}^{\infty} \sum_{l_{1}=0}^{\infty} \sum_{m_{1}=0}^{\infty} k_{1} P_{i_{1} j_{1} k_{1} l_{1} m_{1}} \\
& +k_{t r t} \sum_{i=1}^{\infty} \sum_{j=0}^{\infty} \sum_{k=0}^{\infty} \sum_{l=0}^{\infty} \sum_{m=0}^{\infty} i k P_{i j k l m} \sum_{i_{1}=1}^{\infty} \sum_{j_{1}=0}^{\infty} \sum_{k_{1}=0}^{\infty} \sum_{l_{1}=0}^{\infty} \sum_{m_{1}=0}^{\infty} l_{1} P_{i_{1} j_{1} k_{1} l_{1} m_{1}} \\
& +k_{t r t} \sum_{i=1}^{\infty} \sum_{j=0}^{\infty} \sum_{k=0}^{\infty} \sum_{l=0}^{\infty} \sum_{m=0}^{\infty} i l P_{i j k l m} \sum_{i_{1}=1}^{\infty} \sum_{j_{1}=0}^{\infty} \sum_{k_{1}=0}^{\infty} \sum_{l_{1}=0}^{\infty} \sum_{m_{1}=0}^{\infty} k_{1} P_{i_{1} j_{1} k_{1} l_{1} m_{1}} \\
& -k_{t r p} \sum_{i=1}^{\infty} \sum_{j=0}^{\infty} \sum_{k=0}^{\infty} \sum_{l=0}^{\infty} \sum_{m=0}^{\infty} i k P_{i j k l m} \sum_{i_{1}=1}^{\infty} \sum_{j_{1}=0}^{\infty} \sum_{k_{1}=0}^{\infty} \sum_{l_{1}=0}^{\infty} \sum_{m_{1}=0}^{\infty} i_{1} P_{i_{1} j_{1} k_{1} l_{1} m_{1}} \\
& -k_{\text {trp }} \sum_{i=1}^{\infty} \sum_{j=0}^{\infty} \sum_{k=0}^{\infty} \sum_{l=0}^{\infty} \sum_{m=0}^{\infty} i^{2} P_{i j k l m} \sum_{i_{1}=1}^{\infty} \sum_{j_{1}=0}^{\infty} \sum_{k_{1}=0}^{\infty} \sum_{l_{1}=0}^{\infty} \sum_{m_{1}=0}^{\infty} k_{1} P_{i_{1} j_{1} k_{1} l_{1} m_{1}} \\
& +k_{\text {trp }} \sum_{i=1}^{\infty} \sum_{j=0}^{\infty} \sum_{k=0}^{\infty} \sum_{l=0}^{\infty} \sum_{m=0}^{\infty} i k P_{i j k l m} \sum_{i_{1}=1}^{\infty} \sum_{j_{1}=0}^{\infty} \sum_{k_{1}=0}^{\infty} \sum_{l_{1}=0}^{\infty} \sum_{m_{1}=0}^{\infty} i_{1} P_{i_{1} j_{1} k_{1} l_{1} m_{1}} \\
& +k_{t r p} \sum_{i=1}^{\infty} \sum_{j=0}^{\infty} \sum_{k=0}^{\infty} \sum_{l=0}^{\infty} \sum_{m=0}^{\infty} i^{2} P_{i j k l m} \sum_{i_{1}=1}^{\infty} \sum_{j_{1}=0}^{\infty} \sum_{k_{1}=0}^{\infty} \sum_{l_{1}=0}^{\infty} \sum_{m_{1}=0}^{\infty} k_{1} P_{i_{1} j_{1} k_{1} l_{1} m_{1}}
\end{aligned}
$$




$$
\begin{aligned}
& -k_{t r p} \sum_{i=1}^{\infty} \sum_{j=0}^{\infty} \sum_{k=0}^{\infty} \sum_{l=0}^{\infty} \sum_{m=0}^{\infty} i m P_{i j k l m} \sum_{i_{1}=1}^{\infty} \sum_{j_{1}=0}^{\infty} \sum_{k_{1}=0}^{\infty} \sum_{l_{1}=0}^{\infty} \sum_{m_{1}=0}^{\infty} i_{1} P_{i_{1} j_{1} k_{1} l_{1} m_{1}} \\
& -k_{\operatorname{trp}} \sum_{i=1}^{\infty} \sum_{j=0}^{\infty} \sum_{k=0}^{\infty} \sum_{l=0}^{\infty} \sum_{m=0}^{\infty} i^{2} P_{i j k l m} \sum_{i_{1}=1}^{\infty} \sum_{j_{1}=0}^{\infty} \sum_{k_{1}=0}^{\infty} \sum_{l_{1}=0}^{\infty} \sum_{m_{1}=0}^{\infty} m_{1} P_{i_{1} j_{1} k_{1} l_{1} m_{1}} \\
& +k_{\text {trp }} \sum_{i=1}^{\infty} \sum_{j=0}^{\infty} \sum_{k=0}^{\infty} \sum_{l=0}^{\infty} \sum_{m=0}^{\infty} i m P_{i j k l m} \sum_{i_{1}=1}^{\infty} \sum_{j_{1}=0}^{\infty} \sum_{k_{1}=0}^{\infty} \sum_{l_{1}=0}^{\infty} \sum_{m_{1}=0}^{\infty} i_{1} P_{i_{1} j_{1} k_{1} l_{1} m_{1}} \\
& +k_{t r p} \sum_{i=1}^{\infty} \sum_{j=0}^{\infty} \sum_{k=0}^{\infty} \sum_{l=0}^{\infty} \sum_{m=0}^{\infty} i^{2} P_{i j k l m} \sum_{i_{1}=1}^{\infty} \sum_{j_{1}=0}^{\infty} \sum_{k_{1}=0}^{\infty} \sum_{l_{1}=0}^{\infty} \sum_{m_{1}=0}^{\infty} m_{1} P_{i_{1} j_{1} k_{1} l_{1} m_{1}} \\
& -k_{i n t r} \sum_{i=1}^{\infty} \sum_{j=0}^{\infty} \sum_{k=0}^{\infty} \sum_{l=0}^{\infty} \sum_{m=0}^{\infty} i k P_{i j k l m} \\
& +k_{\text {intr }} \sum_{i=1}^{\infty} \sum_{j=0}^{\infty} \sum_{k=0}^{\infty} \sum_{l=0}^{\infty} \sum_{m=0}^{\infty} i k P_{i j k l m} \\
& -k_{r} \sum_{i=1}^{\infty} \sum_{j=0}^{\infty} \sum_{k=0}^{\infty} \sum_{l=0}^{\infty} \sum_{m=0}^{\infty} i m P_{i j k l m} \\
& +k_{r} \sum_{i=1}^{\infty} \sum_{j=0}^{\infty} \sum_{k=0}^{\infty} \sum_{l=0}^{\infty} \sum_{m=0}^{\infty} i m P_{i j k l m} \\
& -k_{t c} \sum_{i=1}^{\infty} \sum_{j=0}^{\infty} \sum_{k=0}^{\infty} \sum_{l=0}^{\infty} \sum_{m=0}^{\infty} i k P_{i j k l m} \sum_{i_{1}=1}^{\infty} \sum_{j_{1}=0}^{\infty} \sum_{k_{1}=0}^{\infty} \sum_{l_{1}=0}^{\infty} \sum_{m_{1}=0}^{\infty} k_{1} P_{i_{1} j_{1} k_{1} l_{1} m_{1}} \\
& +\frac{1}{2}(2) k_{t c} \sum_{i=1}^{\infty} \sum_{j=0}^{\infty} \sum_{k=0}^{\infty} \sum_{l=0}^{\infty} \sum_{m=0}^{\infty} i k P_{i j k l m} \sum_{i_{1}=1}^{\infty} \sum_{j_{1}=0}^{\infty} \sum_{k_{1}=0}^{\infty} \sum_{l_{1}=0}^{\infty} \sum_{m_{1}=0}^{\infty} k_{1} P_{i_{1} j_{1} k_{1} l_{1} m_{1}} \\
& -k_{t c} \sum_{i=1}^{\infty} \sum_{j=0}^{\infty} \sum_{k=0}^{\infty} \sum_{l=0}^{\infty} \sum_{m=0}^{\infty} i m P_{i j k l m} \sum_{i_{1}=1}^{\infty} \sum_{j_{1}=0}^{\infty} \sum_{k_{1}=0}^{\infty} \sum_{l_{1}=0}^{\infty} \sum_{m_{1}=0}^{\infty} m_{1} P_{i_{1} j_{1} k_{1} l_{1} m_{1}} \\
& +\frac{1}{2}(2) k_{t c} \sum_{i=1}^{\infty} \sum_{j=0}^{\infty} \sum_{k=0}^{\infty} \sum_{l=0}^{\infty} \sum_{m=0}^{\infty} i m P_{i j k l m} \sum_{i_{1}=1}^{\infty} \sum_{j_{1}=0}^{\infty} \sum_{k_{1}=0}^{\infty} \sum_{l_{1}=0}^{\infty} \sum_{m_{1}=0}^{\infty} m_{1} P_{i_{1} j_{1} k_{1} l_{1} m_{1}} \\
& -k_{t c} \sum_{i=1}^{\infty} \sum_{j=0}^{\infty} \sum_{k=0}^{\infty} \sum_{l=0}^{\infty} \sum_{m=0}^{\infty} i k P_{i j k l m} \sum_{i_{1}=1}^{\infty} \sum_{j_{1}=0}^{\infty} \sum_{k_{1}=0}^{\infty} \sum_{l_{1}=0}^{\infty} \sum_{m_{1}=0}^{\infty} m_{1} P_{i_{1} j_{1} k_{1} l_{1} m_{1}} \\
& -k_{t c} \sum_{i=1}^{\infty} \sum_{j=0}^{\infty} \sum_{k=0}^{\infty} \sum_{l=0}^{\infty} \sum_{m=0}^{\infty} i m P_{i j k l m} \sum_{i_{1}=1}^{\infty} \sum_{j_{1}=0}^{\infty} \sum_{k_{1}=0}^{\infty} \sum_{l_{1}=0}^{\infty} \sum_{m_{1}=0}^{\infty} k_{1} P_{i_{1} j_{1} k_{1} l_{1} m_{1}} \\
& +\frac{1}{2}(2) k_{t c} \sum_{i=1}^{\infty} \sum_{j=0}^{\infty} \sum_{k=0}^{\infty} \sum_{l=0}^{\infty} \sum_{m=0}^{\infty} i k P_{i j k l m} \sum_{i_{1}=1}^{\infty} \sum_{j_{1}=0}^{\infty} \sum_{k_{1}=0}^{\infty} \sum_{l_{1}=0}^{\infty} \sum_{m_{1}=0}^{\infty} m_{1} P_{i_{1} j_{1} k_{1} l_{1} m_{1}} \\
& +\frac{1}{2}(2) k_{t c} \sum_{i=1}^{\infty} \sum_{j=0}^{\infty} \sum_{k=0}^{\infty} \sum_{l=0}^{\infty} \sum_{m=0}^{\infty} i m P_{i j k l m} \sum_{i_{1}=1}^{\infty} \sum_{j_{1}=0}^{\infty} \sum_{k_{1}=0}^{\infty} \sum_{l_{1}=0}^{\infty} \sum_{m_{1}=0}^{\infty} k_{1} P_{i_{1} j_{1} k_{1} l_{1} m_{1}}
\end{aligned}
$$

using Equations (42) and (43). 


$$
\begin{aligned}
& \Rightarrow \frac{d \mu_{10000}}{d t}=k_{i} C_{M} C_{R} \\
& \quad+k_{p} C_{M} \sum_{i=1}^{\infty} \sum_{j=0}^{\infty} \sum_{k=0}^{\infty} \sum_{l=0}^{\infty} \sum_{m=0}^{\infty} k P_{i j k l m} \\
& \quad+k_{p} C_{M} \sum_{i=1}^{\infty} \sum_{j=0}^{\infty} \sum_{k=0}^{\infty} \sum_{l=0}^{\infty} \sum_{m=0}^{\infty} m P_{i j k l m} \\
& \Rightarrow \frac{d \mu_{10000}}{d t}=k_{i} C_{M} C_{R}+k_{p} C_{M} \mu_{00100}+k_{p} C_{M} \mu_{00001}
\end{aligned}
$$




$$
\begin{aligned}
& \frac{d \mu_{01000}}{d t}=\sum_{i=1}^{\infty} \sum_{j=0}^{\infty} \sum_{k=0}^{\infty} \sum_{l=0}^{\infty} \sum_{m=0}^{\infty} j \frac{d P_{i j k l m}}{d t} \\
& =-k_{p} C_{M} \sum_{i=1}^{\infty} \sum_{j=0}^{\infty} \sum_{k=0}^{\infty} \sum_{l=0}^{\infty} \sum_{m=0}^{\infty} j k P_{i j k l m} \\
& +k_{p} C_{M} \sum_{i=2}^{\infty} \sum_{j=0}^{\infty} \sum_{k=0}^{\infty} \sum_{l=0}^{\infty} \sum_{m=0}^{\infty} j k P_{i-1 j k l m} \\
& -k_{p} C_{M} \sum_{i=1}^{\infty} \sum_{j=0}^{\infty} \sum_{k=0}^{\infty} \sum_{l=0}^{\infty} \sum_{m=0}^{\infty} j m P_{i j k l m} \\
& +k_{p} C_{M} \sum_{i=2}^{\infty} \sum_{j=0}^{\infty} \sum_{k=0}^{\infty} \sum_{l=0}^{\infty} \sum_{m=0}^{\infty} j m P_{i-1 j k l m} \\
& -k_{t r t} C_{T} \sum_{i=1}^{\infty} \sum_{j=0}^{\infty} \sum_{k=0}^{\infty} \sum_{l=0}^{\infty} \sum_{m=0}^{\infty} j k P_{i j k l m} \\
& +k_{t r t} C_{T} \sum_{i=1}^{\infty} \sum_{j=0}^{\infty} \sum_{k=0}^{\infty} \sum_{l=1}^{\infty} \sum_{m=0}^{\infty} j(k+1) P_{i j k+1 l-1 m} \\
& -k_{t r t} \sum_{i=1}^{\infty} \sum_{j=0}^{\infty} \sum_{k=0}^{\infty} \sum_{l=0}^{\infty} \sum_{m=0}^{\infty} j k P_{i j k l m} \sum_{i_{1}=1}^{\infty} \sum_{j_{1}=0}^{\infty} \sum_{k_{1}=0}^{\infty} \sum_{l_{1}=0}^{\infty} \sum_{m_{1}=0}^{\infty} l_{1} P_{i_{1} j_{1} k_{1} l_{1} m_{1}} \\
& -k_{t r t} \sum_{i=1}^{\infty} \sum_{j=0}^{\infty} \sum_{k=0}^{\infty} \sum_{l=0}^{\infty} \sum_{m=0}^{\infty} j l P_{i j k l m} \sum_{i_{1}=1}^{\infty} \sum_{j_{1}=0}^{\infty} \sum_{k_{1}=0}^{\infty} \sum_{l_{1}=0}^{\infty} \sum_{m_{1}=0}^{\infty} k_{1} P_{i_{1} j_{1} k_{1} l_{1} m_{1}} \\
& +k_{t r t} \sum_{i=1}^{\infty} \sum_{j=0}^{\infty} \sum_{k=0}^{\infty} \sum_{l=1}^{\infty} \sum_{m=0}^{\infty} j(k+1) P_{i j k+1 l-1 m} \sum_{i_{1}=1}^{\infty} \sum_{j_{1}=0}^{\infty} \sum_{k_{1}=0}^{\infty} \sum_{l_{1}=0}^{\infty} \sum_{m_{1}=0}^{\infty} l_{1} P_{i_{1} j_{1} k_{1} l_{1} m_{1}} \\
& +k_{t r t} \sum_{i=1}^{\infty} \sum_{j=0}^{\infty} \sum_{k=0}^{\infty} \sum_{l=0}^{\infty} \sum_{m=1}^{\infty} j(l+1) P_{i j k l+1 m-1} \sum_{i_{1}=1}^{\infty} \sum_{j_{1}=0}^{\infty} \sum_{k_{1}=0}^{\infty} \sum_{l_{1}=0}^{\infty} \sum_{m_{1}=0}^{\infty} k_{1} P_{i_{1} j_{1} k_{1} l_{1} m_{1}} \\
& -k_{\operatorname{trp}} \sum_{i=1}^{\infty} \sum_{j=0}^{\infty} \sum_{k=0}^{\infty} \sum_{l=0}^{\infty} \sum_{m=0}^{\infty} j k P_{i j k l m} \sum_{i_{1}=1}^{\infty} \sum_{j_{1}=0}^{\infty} \sum_{k_{1}=0}^{\infty} \sum_{l_{1}=0}^{\infty} \sum_{m_{1}=0}^{\infty} i_{1} P_{i_{1} j_{1} k_{1} l_{1} m_{1}} \\
& -k_{\operatorname{trp}} \sum_{i=1}^{\infty} \sum_{j=0}^{\infty} \sum_{k=0}^{\infty} \sum_{l=0}^{\infty} \sum_{m=0}^{\infty} i j P_{i j k l m} \sum_{i_{1}=1}^{\infty} \sum_{j_{1}=0}^{\infty} \sum_{k_{1}=0}^{\infty} \sum_{l_{1}=0}^{\infty} \sum_{m_{1}=0}^{\infty} k_{1} P_{i_{1} j_{1} k_{1} l_{1} m_{1}} \\
& +k_{\operatorname{trp}} \sum_{i=1}^{\infty} \sum_{j=0}^{\infty} \sum_{k=0}^{\infty} \sum_{l=0}^{\infty} \sum_{m=0}^{\infty} j(k+1) P_{i j k+1 l m} \sum_{i_{1}=1}^{\infty} \sum_{j_{1}=0}^{\infty} \sum_{k_{1}=0}^{\infty} \sum_{l_{1}=0}^{\infty} \sum_{m_{1}=0}^{\infty} i_{1} P_{i_{1} j_{1} k_{1} l_{1} m_{1}} \\
& +k_{\operatorname{trp}} \sum_{i=1}^{\infty} \sum_{j=1}^{\infty} \sum_{k=1}^{\infty} \sum_{l=0}^{\infty} \sum_{m=0}^{\infty} i j P_{i j-1 k-1 l m} \sum_{i_{1}=1}^{\infty} \sum_{j_{1}=0}^{\infty} \sum_{k_{1}=0}^{\infty} \sum_{l_{1}=0}^{\infty} \sum_{m_{1}=0}^{\infty} k_{1} P_{i_{1} j_{1} k_{1} l_{1} m_{1}}
\end{aligned}
$$




$$
\begin{aligned}
& -k_{t r p} \sum_{i=1}^{\infty} \sum_{j=0}^{\infty} \sum_{k=0}^{\infty} \sum_{l=0}^{\infty} \sum_{m=0}^{\infty} j m P_{i j k l m} \sum_{i_{1}=1}^{\infty} \sum_{j_{1}=0}^{\infty} \sum_{k_{1}=0}^{\infty} \sum_{l_{1}=0}^{\infty} \sum_{m_{1}=0}^{\infty} i_{1} P_{i_{1} j_{1} k_{1} l_{1} m_{1}} \\
& -k_{t r p} \sum_{i=1}^{\infty} \sum_{j=0}^{\infty} \sum_{k=0}^{\infty} \sum_{l=0}^{\infty} \sum_{m=0}^{\infty} i j P_{i j k l m} \sum_{i_{1}=1}^{\infty} \sum_{j_{1}=0}^{\infty} \sum_{k_{1}=0}^{\infty} \sum_{l_{1}=0}^{\infty} \sum_{m_{1}=0}^{\infty} m_{1} P_{i_{1} j_{1} k_{1} l_{1} m_{1}} \\
& +k_{\operatorname{trp}} \sum_{i=1}^{\infty} \sum_{j=0}^{\infty} \sum_{k=0}^{\infty} \sum_{l=0}^{\infty} \sum_{m=0}^{\infty} j(m+1) P_{i j k l m+1} \sum_{i_{1}=1}^{\infty} \sum_{j_{1}=0}^{\infty} \sum_{k_{1}=0}^{\infty} \sum_{l_{1}=0}^{\infty} \sum_{m_{1}=0}^{\infty} i_{1} P_{i_{1} j_{1} k_{1} l_{1} m_{1}} \\
& +k_{\text {trp }} \sum_{i=1}^{\infty} \sum_{j=1}^{\infty} \sum_{k=0}^{\infty} \sum_{l=0}^{\infty} \sum_{m=1}^{\infty} i j P_{i j-1 k l m-1} \sum_{i_{1}=1}^{\infty} \sum_{j_{1}=0}^{\infty} \sum_{k_{1}=0}^{\infty} \sum_{l_{1}=0}^{\infty} \sum_{m_{1}=0}^{\infty} m_{1} P_{i_{1} j_{1} k_{1} l_{1} m_{1}} \\
& -k_{i n t r} \sum_{i=1}^{\infty} \sum_{j=0}^{\infty} \sum_{k=0}^{\infty} \sum_{l=0}^{\infty} \sum_{m=0}^{\infty} j k P_{i j k l m} \\
& +k_{i n t r} \sum_{i=1}^{\infty} \sum_{j=1}^{\infty} \sum_{k=0}^{\infty} \sum_{l=0}^{\infty} \sum_{m=0}^{\infty} j k P_{i j-1 k l m} \\
& -k_{r} \sum_{i=1}^{\infty} \sum_{j=0}^{\infty} \sum_{k=0}^{\infty} \sum_{l=0}^{\infty} \sum_{m=0}^{\infty} j m P_{i j k l m} \\
& +k_{r} \sum_{i=1}^{\infty} \sum_{j=0}^{\infty} \sum_{k=1}^{\infty} \sum_{l=0}^{\infty} \sum_{m=0}^{\infty} j(m+1) P_{i j k-1 l m+1} \\
& -k_{t c} \sum_{i=1}^{\infty} \sum_{j=0}^{\infty} \sum_{k=0}^{\infty} \sum_{l=0}^{\infty} \sum_{m=0}^{\infty} j k P_{i j k l m} \sum_{i_{1}=1}^{\infty} \sum_{j_{1}=0}^{\infty} \sum_{k_{1}=0}^{\infty} \sum_{l_{1}=0}^{\infty} \sum_{m_{1}=0}^{\infty} k_{1} P_{i_{1} j_{1} k_{1} l_{1} m_{1}} \\
& +\frac{1}{2} k_{t c} \sum_{i=1}^{\infty} \sum_{j=0}^{\infty} \sum_{k=0}^{\infty} \sum_{l=0}^{\infty} \sum_{m=0}^{\infty} \sum_{i_{1}=1}^{i} \sum_{j_{1}=0}^{j} \sum_{k_{1}=0}^{k+2} \sum_{l_{1}=0}^{l} \sum_{m_{1}=0}^{m} j\left[k_{1} P_{i_{1} j_{1} k_{1} l_{1} m_{1}}\left(k+2-k_{1}\right) P_{i-i_{1}, j-j_{1}, k+2-k_{1}, l-l_{1} m-m_{1}}\right] \\
& -k_{t c} \sum_{i=1}^{\infty} \sum_{j=0}^{\infty} \sum_{k=0}^{\infty} \sum_{l=0}^{\infty} \sum_{m=0}^{\infty} j m P_{i j k l m} \sum_{i_{1}=1}^{\infty} \sum_{j_{1}=0}^{\infty} \sum_{k_{1}=0}^{\infty} \sum_{l_{1}=0}^{\infty} \sum_{m_{1}=0}^{\infty} m_{1} P_{i_{1} j_{1} k_{1} l_{1} m_{1}} \\
& +\frac{1}{2} k_{t c} \sum_{i=1}^{\infty} \sum_{j=0}^{\infty} \sum_{k=0}^{\infty} \sum_{l=0}^{\infty} \sum_{m=0}^{\infty} \sum_{i_{1}=1}^{i} \sum_{j_{1}=0}^{j} \sum_{k_{1}=0}^{k} \sum_{l_{1}=0}^{l} \sum_{m_{1}=0}^{m+2}\left[j m_{1} P_{i_{1} j_{1} k_{1} l_{1} m_{1}}\left(m+2-m_{1}\right) P_{i-i_{1}, j-j_{1}, k-k_{1}, l-l_{1} m+2-m_{1}}\right] \\
& -k_{t c} \sum_{i=1}^{\infty} \sum_{j=0}^{\infty} \sum_{k=0}^{\infty} \sum_{l=0}^{\infty} \sum_{m=0}^{\infty} j k P_{i j k l m} \sum_{i_{1}=1}^{\infty} \sum_{j_{1}=0}^{\infty} \sum_{k_{1}=0}^{\infty} \sum_{l_{1}=0}^{\infty} \sum_{m_{1}=0}^{\infty} m_{1} P_{i_{1} j_{1} k_{1} l_{1} m_{1}} \\
& -k_{t c} \sum_{i=1}^{\infty} \sum_{j=0}^{\infty} \sum_{k=0}^{\infty} \sum_{l=0}^{\infty} \sum_{m=0}^{\infty} j m P_{i j k l m} \sum_{i_{1}=1}^{\infty} \sum_{j_{1}=0}^{\infty} \sum_{k_{1}=0}^{\infty} \sum_{l_{1}=0}^{\infty} \sum_{m_{1}=0}^{\infty} k_{1} P_{i_{1} j_{1} k_{1} l_{1} m_{1}} \\
& +\frac{1}{2} k_{t c} \sum_{i=1}^{\infty} \sum_{j=0}^{\infty} \sum_{k=0}^{\infty} \sum_{l=0}^{\infty} \sum_{m=0}^{\infty} \sum_{i_{1}=1}^{i} \sum_{j_{1}=0}^{j} \sum_{k_{1}=0}^{k+1} \sum_{l_{1}=0}^{l} \sum_{m_{1}=0}^{m+1}\left[j k_{1} P_{i_{1} j_{1} k_{1} l_{1} m_{1}}\left(m+1-m_{1}\right) P_{\left.i-i_{1}, j-j_{1}, k+1-k_{1}, l-l_{1} m+1-m_{1}\right]}\right. \\
& +\frac{1}{2} k_{t c} \sum_{i=1}^{\infty} \sum_{j=0}^{\infty} \sum_{k=0}^{\infty} \sum_{l=0}^{\infty} \sum_{m=0}^{\infty} \sum_{i_{1}=1}^{i} \sum_{j_{1}=0}^{j} \sum_{k_{1}=0}^{k+1} \sum_{l_{1}=0}^{l} \sum_{m_{1}=0}^{m+1}\left[j m_{1} P_{i_{1} j_{1} k_{1} l_{1} m_{1}}\left(k+1-k_{1}\right) P_{i-i_{1}, j-j_{1}, k+1-k_{1}, l-l_{1} m+1-m_{1}}\right]
\end{aligned}
$$




$$
\begin{aligned}
& \Rightarrow \frac{d \mu_{01000}}{d t}=-k_{p} C_{M} \sum_{i=1}^{\infty} \sum_{j=0}^{\infty} \sum_{k=0}^{\infty} \sum_{l=0}^{\infty} \sum_{m=0}^{\infty} j k P_{i j k l m} \\
& +k_{p} C_{M} \sum_{i=1}^{\infty} \sum_{j=0}^{\infty} \sum_{k=0}^{\infty} \sum_{l=0}^{\infty} \sum_{m=0}^{\infty} j k P_{i j k l m} \\
& -k_{p} C_{M} \sum_{i=1}^{\infty} \sum_{j=0}^{\infty} \sum_{k=0}^{\infty} \sum_{l=0}^{\infty} \sum_{m=0}^{\infty} j m P_{i j k l m} \\
& +k_{p} C_{M} \sum_{i=1}^{\infty} \sum_{j=0}^{\infty} \sum_{k=0}^{\infty} \sum_{l=0}^{\infty} \sum_{m=0}^{\infty} j m P_{i j k l m} \\
& -k_{t r t} C_{T} \sum_{i=1}^{\infty} \sum_{j=0}^{\infty} \sum_{k=0}^{\infty} \sum_{l=0}^{\infty} \sum_{m=0}^{\infty} j k P_{i j k l m} \\
& +k_{t r t} C_{T} \sum_{i=1}^{\infty} \sum_{j=0}^{\infty} \sum_{k=0}^{\infty} \sum_{l=0}^{\infty} \sum_{m=0}^{\infty} j k P_{i j k l m} \\
& -k_{f i} C_{I} \sum_{i=1}^{\infty} \sum_{j=0}^{\infty} \sum_{k=0}^{\infty} \sum_{l=0}^{\infty} \sum_{m=0}^{\infty} j m P_{i j k l m} \\
& +k_{f i} C_{I} \sum_{i=1}^{\infty} \sum_{j=0}^{\infty} \sum_{k=0}^{\infty} \sum_{l=0}^{\infty} \sum_{m=0}^{\infty} j m P_{i j k l m} \\
& \text { - } k_{t r t} \sum_{i=1}^{\infty} \sum_{j=0}^{\infty} \sum_{k=0}^{\infty} \sum_{l=0}^{\infty} \sum_{m=0}^{\infty} j k P_{i j k l m} \sum_{i_{1}=1}^{\infty} \sum_{j_{1}=0}^{\infty} \sum_{k_{1}=0}^{\infty} \sum_{l_{1}=0}^{\infty} \sum_{m_{1}=0}^{\infty} l_{1} P_{i_{1} j_{1}} k_{1} l_{1} m_{1} \\
& \text { - } k_{\text {trt }} \sum_{i=1}^{\infty} \sum_{j=0}^{\infty} \sum_{k=0}^{\infty} \sum_{l=0}^{\infty} \sum_{m=0}^{\infty} j l P_{i j k l m} \sum_{i_{1}=1}^{\infty} \sum_{j_{1}=0}^{\infty} \sum_{k_{1}=0}^{\infty} \sum_{l_{1}=0}^{\infty} \sum_{m_{1}=0}^{\infty} k_{1} P_{i_{1} j_{1} k_{1} l_{1} m_{1}} \\
& +k_{t r t} \sum_{i=1}^{\infty} \sum_{j=0}^{\infty} \sum_{k=0}^{\infty} \sum_{l=0}^{\infty} \sum_{m=0}^{\infty} j k P_{i j k l m} \sum_{i_{1}=1}^{\infty} \sum_{j_{1}=0}^{\infty} \sum_{k_{1}=0}^{\infty} \sum_{l_{1}=0}^{\infty} \sum_{m_{1}=0}^{\infty} l_{1} P_{i_{1} j_{1} k_{1} l_{1} m_{1}} \\
& +k_{t r t} \sum_{i=1}^{\infty} \sum_{j=0}^{\infty} \sum_{k=0}^{\infty} \sum_{l=0}^{\infty} \sum_{m=0}^{\infty} j l P_{i j k l m} \sum_{i_{1}=1}^{\infty} \sum_{j_{1}=0}^{\infty} \sum_{k_{1}=0}^{\infty} \sum_{l_{1}=0}^{\infty} \sum_{m_{1}=0}^{\infty} k_{1} P_{i_{1} j_{1} k_{1} l_{1} m_{1}} \\
& -k_{t r p} \sum_{i=1}^{\infty} \sum_{j=0}^{\infty} \sum_{k=0}^{\infty} \sum_{l=0}^{\infty} \sum_{m=0}^{\infty} j k P_{i j k l m} \sum_{i_{1}=1}^{\infty} \sum_{j_{1}=0}^{\infty} \sum_{k_{1}=0}^{\infty} \sum_{l_{1}=0}^{\infty} \sum_{m_{1}=0}^{\infty} i_{1} P_{i_{1} j_{1} k_{1} l_{1} m_{1}} \\
& -k_{t r p} \sum_{i=1}^{\infty} \sum_{j=0}^{\infty} \sum_{k=0}^{\infty} \sum_{l=0}^{\infty} \sum_{m=0}^{\infty} i j P_{i j k l m} \sum_{i_{1}=1}^{\infty} \sum_{j_{1}=0}^{\infty} \sum_{k_{1}=0}^{\infty} \sum_{l_{1}=0}^{\infty} \sum_{m_{1}=0}^{\infty} k_{1} P_{i_{1} j_{1} k_{1} l_{1} m_{1}} \\
& +k_{\text {trp }} \sum_{i=1}^{\infty} \sum_{j=0}^{\infty} \sum_{k=0}^{\infty} \sum_{l=0}^{\infty} \sum_{m=0}^{\infty} j k P_{i j k l m} \sum_{i_{1}=1}^{\infty} \sum_{j_{1}=0}^{\infty} \sum_{k_{1}=0}^{\infty} \sum_{l_{1}=0}^{\infty} \sum_{m_{1}=0}^{\infty} i_{1} P_{i_{1} j_{1} k_{1} l_{1} m_{1}} \\
& +k_{t r p} \sum_{i=1}^{\infty} \sum_{j=0}^{\infty} \sum_{k=0}^{\infty} \sum_{l=0}^{\infty} \sum_{m=0}^{\infty} i(j+1) P_{i j k l m} \sum_{i_{1}=1}^{\infty} \sum_{j_{1}=0}^{\infty} \sum_{k_{1}=0}^{\infty} \sum_{l_{1}=0}^{\infty} \sum_{m_{1}=0}^{\infty} k_{1} P_{i_{1} j_{1} k_{1} l_{1} m_{1}}
\end{aligned}
$$




$$
\begin{aligned}
& -k_{\operatorname{trp}} \sum_{i=1}^{\infty} \sum_{j=0}^{\infty} \sum_{k=0}^{\infty} \sum_{l=0}^{\infty} \sum_{m=0}^{\infty} j m P_{i j k l m} \sum_{i_{1}=1}^{\infty} \sum_{j_{1}=0}^{\infty} \sum_{k_{1}=0}^{\infty} \sum_{l_{1}=0}^{\infty} \sum_{m_{1}=0}^{\infty} i_{1} P_{i_{1} j_{1} k_{1} l_{1} m_{1}} \\
& -k_{\operatorname{trp}} \sum_{i=1}^{\infty} \sum_{j=0}^{\infty} \sum_{k=0}^{\infty} \sum_{l=0}^{\infty} \sum_{m=0}^{\infty} i j P_{i j k l m} \sum_{i_{1}=1}^{\infty} \sum_{j_{1}=0}^{\infty} \sum_{k_{1}=0}^{\infty} \sum_{l_{1}=0}^{\infty} \sum_{m_{1}=0}^{\infty} m_{1} P_{i_{1} j_{1} k_{1} l_{1} m_{1}} \\
& +k_{\text {trp }} \sum_{i=1}^{\infty} \sum_{j=0}^{\infty} \sum_{k=0}^{\infty} \sum_{l=0}^{\infty} \sum_{m=0}^{\infty} j m P_{i j k l m} \sum_{i_{1}=1}^{\infty} \sum_{j_{1}=0}^{\infty} \sum_{k_{1}=0}^{\infty} \sum_{l_{1}=0}^{\infty} \sum_{m_{1}=0}^{\infty} i_{1} P_{i_{1} j_{1} k_{1} l_{1} m_{1}} \\
& +k_{t r p} \sum_{i=1}^{\infty} \sum_{j=0}^{\infty} \sum_{k=0}^{\infty} \sum_{l=0}^{\infty} \sum_{m=0}^{\infty} i(j+1) P_{i j k l m} \sum_{i_{1}=1}^{\infty} \sum_{j_{1}=0}^{\infty} \sum_{k_{1}=0}^{\infty} \sum_{l_{1}=0}^{\infty} \sum_{m_{1}=0}^{\infty} m_{1} P_{i_{1} j_{1} k_{1} l_{1} m_{1}} \\
& -k_{i n t r} \sum_{i=1}^{\infty} \sum_{j=0}^{\infty} \sum_{k=0}^{\infty} \sum_{l=0}^{\infty} \sum_{m=0}^{\infty} j k P_{i j k l m} \\
& +k_{i n t r} \sum_{i=1}^{\infty} \sum_{j=0}^{\infty} \sum_{k=0}^{\infty} \sum_{l=0}^{\infty} \sum_{m=0}^{\infty}(j+1) k P_{i j k l m} \\
& -k_{r} \sum_{i=1}^{\infty} \sum_{j=0}^{\infty} \sum_{k=0}^{\infty} \sum_{l=0}^{\infty} \sum_{m=0}^{\infty} j m P_{i j k l m} \\
& +k_{r} \sum_{i=1}^{\infty} \sum_{j=0}^{\infty} \sum_{k=0}^{\infty} \sum_{l=0}^{\infty} \sum_{m=0}^{\infty} j m P_{i j k l m} \\
& -k_{t c} \sum_{i=1}^{\infty} \sum_{j=0}^{\infty} \sum_{k=0}^{\infty} \sum_{l=0}^{\infty} \sum_{m=0}^{\infty} j k P_{i j k l m} \sum_{i_{1}=1}^{\infty} \sum_{j_{1}=0}^{\infty} \sum_{k_{1}=0}^{\infty} \sum_{l_{1}=0}^{\infty} \sum_{m_{1}=0}^{\infty} k_{1} P_{i_{1} j_{1} k_{1} l_{1} m_{1}} \\
& +\frac{1}{2}(2) k_{t c} \sum_{i=1}^{\infty} \sum_{j=0}^{\infty} \sum_{k=0}^{\infty} \sum_{l=0}^{\infty} \sum_{m=0}^{\infty} j k P_{i j k l m} \sum_{i_{1}=1}^{\infty} \sum_{j_{1}=0}^{\infty} \sum_{k_{1}=0}^{\infty} \sum_{l_{1}=0}^{\infty} \sum_{m_{1}=0}^{\infty} k_{1} P_{i_{1} j_{1} k_{1} l_{1} m_{1}} \\
& -k_{t c} \sum_{i=1}^{\infty} \sum_{j=0}^{\infty} \sum_{k=0}^{\infty} \sum_{l=0}^{\infty} \sum_{m=0}^{\infty} j m P_{i j k l m} \sum_{i_{1}=1}^{\infty} \sum_{j_{1}=0}^{\infty} \sum_{k_{1}=0}^{\infty} \sum_{l_{1}=0}^{\infty} \sum_{m_{1}=0}^{\infty} m_{1} P_{i_{1} j_{1} k_{1} l_{1} m_{1}} \\
& +\frac{1}{2}(2) k_{t c} \sum_{i=1}^{\infty} \sum_{j=0}^{\infty} \sum_{k=0}^{\infty} \sum_{l=0}^{\infty} \sum_{m=0}^{\infty} j m P_{i j k l m} \sum_{i_{1}=1}^{\infty} \sum_{j_{1}=0}^{\infty} \sum_{k_{1}=0}^{\infty} \sum_{l_{1}=0}^{\infty} \sum_{m_{1}=0}^{\infty} m_{1} P_{i_{1} j_{1} k_{1} l_{1} m_{1}} \\
& -k_{t c} \sum_{i=1}^{\infty} \sum_{j=0}^{\infty} \sum_{k=0}^{\infty} \sum_{l=0}^{\infty} \sum_{m=0}^{\infty} j k P_{i j k l m} \sum_{i_{1}=1}^{\infty} \sum_{j_{1}=0}^{\infty} \sum_{k_{1}=0}^{\infty} \sum_{l_{1}=0}^{\infty} \sum_{m_{1}=0}^{\infty} m_{1} P_{i_{1} j_{1} k_{1} l_{1} m_{1}} \\
& -k_{t c} \sum_{i=1}^{\infty} \sum_{j=0}^{\infty} \sum_{k=0}^{\infty} \sum_{l=0}^{\infty} \sum_{m=0}^{\infty} j m P_{i j k l m} \sum_{i_{1}=1}^{\infty} \sum_{j_{1}=0}^{\infty} \sum_{k_{1}=0}^{\infty} \sum_{l_{1}=0}^{\infty} \sum_{m_{1}=0}^{\infty} k_{1} P_{i_{1} j_{1} k_{1} l_{1} m_{1}} \\
& +\frac{1}{2}(2) k_{t c} \sum_{i=1}^{\infty} \sum_{j=0}^{\infty} \sum_{k=0}^{\infty} \sum_{l=0}^{\infty} \sum_{m=0}^{\infty} j k P_{i j k l m} \sum_{i_{1}=1}^{\infty} \sum_{j_{1}=0}^{\infty} \sum_{k_{1}=0}^{\infty} \sum_{l_{1}=0}^{\infty} \sum_{m_{1}=0}^{\infty} m_{1} P_{i_{1} j_{1} k_{1} l_{1} m_{1}} \\
& +\frac{1}{2}(2) k_{t c} \sum_{i=1}^{\infty} \sum_{j=0}^{\infty} \sum_{k=0}^{\infty} \sum_{l=0}^{\infty} \sum_{m=0}^{\infty} j m P_{i j k l m} \sum_{i_{1}=1}^{\infty} \sum_{j_{1}=0}^{\infty} \sum_{k_{1}=0}^{\infty} \sum_{l_{1}=0}^{\infty} \sum_{m_{1}=0}^{\infty} k_{1} P_{i_{1} j_{1} k_{1} l_{1} m_{1}}
\end{aligned}
$$

using Equations (42) and (43). 


$$
\begin{aligned}
& \Rightarrow \frac{d \mu_{01000}}{d t}=k_{\text {trp }} \sum_{i=1}^{\infty} \sum_{j=0}^{\infty} \sum_{k=0}^{\infty} \sum_{l=0}^{\infty} \sum_{m=0}^{\infty} i P_{i j k l m} \sum_{i_{1}=1}^{\infty} \sum_{j_{1}=0}^{\infty} \sum_{k_{1}=0}^{\infty} \sum_{l_{1}=0}^{\infty} \sum_{m_{1}=0}^{\infty} k_{1} P_{i_{1} j_{1} k_{1} l_{1} m_{1}} \\
& \quad+k_{\text {trp }} \sum_{i=1}^{\infty} \sum_{j=0}^{\infty} \sum_{k=0}^{\infty} \sum_{l=0}^{\infty} \sum_{m=0}^{\infty} i P_{i j k l m} \sum_{i_{1}=1}^{\infty} \sum_{j_{1}=0}^{\infty} \sum_{k_{1}=0}^{\infty} \sum_{l_{1}=0}^{\infty} \sum_{m_{1}=0}^{\infty} m_{1} P_{i_{1} j_{1} k_{1} l_{1} m_{1}} \\
& \quad+k_{\text {intr }} \sum_{i=1}^{\infty} \sum_{j=0}^{\infty} \sum_{k=0}^{\infty} \sum_{l=0}^{\infty} \sum_{m=0}^{\infty} k P_{i j k l m}
\end{aligned}
$$

$\frac{d \mu_{01000}}{d t}=k_{\text {trp }} \mu_{10000} \mu_{00100}+k_{\text {trp }} \mu_{10000} \mu_{00001}+k_{\text {intr }} \mu_{00100}$ 
3.2.3 First moment w.r.t. the number of active (uncapped) radicals

$$
\begin{aligned}
& \frac{d \mu_{00100}}{d t}=\sum_{i=1}^{\infty} \sum_{j=0}^{\infty} \sum_{k=0}^{\infty} \sum_{l=0}^{\infty} \sum_{m=0}^{\infty} k \frac{d P_{i j k l m}}{d t} \\
& =k_{i} C_{M} C_{R} \\
& -k_{p} C_{M} \sum_{i=1}^{\infty} \sum_{j=0}^{\infty} \sum_{k=0}^{\infty} \sum_{l=0}^{\infty} \sum_{m=0}^{\infty} k^{2} P_{i j k l m} \\
& +k_{p} C_{M} \sum_{i=2}^{\infty} \sum_{j=0}^{\infty} \sum_{k=0}^{\infty} \sum_{l=0}^{\infty} \sum_{m=0}^{\infty} k^{2} P_{i-1 j k l m} \\
& -k_{p} C_{M} \sum_{i=1}^{\infty} \sum_{j=0}^{\infty} \sum_{k=0}^{\infty} \sum_{l=0}^{\infty} \sum_{m=0}^{\infty} k m P_{i j k l m} \\
& +k_{p} C_{M} \sum_{i=2}^{\infty} \sum_{j=0}^{\infty} \sum_{k=0}^{\infty} \sum_{l=0}^{\infty} \sum_{m=0}^{\infty} k m P_{i-1 j k l m} \\
& -k_{t r t} C_{T} \sum_{i=1}^{\infty} \sum_{j=0}^{\infty} \sum_{k=0}^{\infty} \sum_{l=0}^{\infty} \sum_{m=0}^{\infty} k^{2} P_{i j k l m} \\
& +k_{t r t} C_{T} \sum_{i=1}^{\infty} \sum_{j=0}^{\infty} \sum_{k=0}^{\infty} \sum_{l=1}^{\infty} \sum_{m=0}^{\infty} k(k+1) P_{i j k+1 l-1 m} \\
& -k_{t r t} \sum_{i=1}^{\infty} \sum_{j=0}^{\infty} \sum_{k=0}^{\infty} \sum_{l=0}^{\infty} \sum_{m=0}^{\infty} k^{2} P_{i j k l m} \sum_{i_{1}=1}^{\infty} \sum_{j_{1}=0}^{\infty} \sum_{k_{1}=0}^{\infty} \sum_{l_{1}=0}^{\infty} \sum_{m_{1}=0}^{\infty} l_{1} P_{i_{1} j_{1} k_{1} l_{1} m_{1}} \\
& -k_{t r t} \sum_{i=1}^{\infty} \sum_{j=0}^{\infty} \sum_{k=0}^{\infty} \sum_{l=0}^{\infty} \sum_{m=0}^{\infty} k l P_{i j k l m} \sum_{i_{1}=1}^{\infty} \sum_{j_{1}=0}^{\infty} \sum_{k_{1}=0}^{\infty} \sum_{l_{1}=0}^{\infty} \sum_{m_{1}=0}^{\infty} k_{1} P_{i_{1} j_{1} k_{1} l_{1} m_{1}} \\
& +k_{t r t} \sum_{i=1}^{\infty} \sum_{j=0}^{\infty} \sum_{k=0}^{\infty} \sum_{l=1}^{\infty} \sum_{m=0}^{\infty} k(k+1) P_{i j k+1 l-1 m} \sum_{i_{1}=1}^{\infty} \sum_{j_{1}=0}^{\infty} \sum_{k_{1}=0}^{\infty} \sum_{l_{1}=0}^{\infty} \sum_{m_{1}=0}^{\infty} l_{1} P_{i_{1} j_{1} k_{1} l_{1} m_{1}} \\
& +k_{t r t} \sum_{i=1}^{\infty} \sum_{j=0}^{\infty} \sum_{k=0}^{\infty} \sum_{l=0}^{\infty} \sum_{m=1}^{\infty} k(l+1) P_{i j k l+1 m-1} \sum_{i_{1}=1}^{\infty} \sum_{j_{1}=0}^{\infty} \sum_{k_{1}=0}^{\infty} \sum_{l_{1}=0}^{\infty} \sum_{m_{1}=0}^{\infty} k_{1} P_{i_{1} j_{1} k_{1} l_{1} m_{1}} \\
& -k_{\operatorname{trp}} \sum_{i=1}^{\infty} \sum_{j=0}^{\infty} \sum_{k=0}^{\infty} \sum_{l=0}^{\infty} \sum_{m=0}^{\infty} k^{2} P_{i j k l m} \sum_{i_{1}=1}^{\infty} \sum_{j_{1}=0}^{\infty} \sum_{k_{1}=0}^{\infty} \sum_{l_{1}=0}^{\infty} \sum_{m_{1}=0}^{\infty} i_{1} P_{i_{1} j_{1} k_{1} l_{1} m_{1}} \\
& -k_{\operatorname{trp}} \sum_{i=1}^{\infty} \sum_{j=0}^{\infty} \sum_{k=0}^{\infty} \sum_{l=0}^{\infty} \sum_{m=0}^{\infty} i k P_{i j k l m} \sum_{i_{1}=1}^{\infty} \sum_{j_{1}=0}^{\infty} \sum_{k_{1}=0}^{\infty} \sum_{l_{1}=0}^{\infty} \sum_{m_{1}=0}^{\infty} k_{1} P_{i_{1} j_{1} k_{1} l_{1} m_{1}} \\
& +k_{\operatorname{trp}} \sum_{i=1}^{\infty} \sum_{j=0}^{\infty} \sum_{k=0}^{\infty} \sum_{l=0}^{\infty} \sum_{m=0}^{\infty} k(k+1) P_{i j k+1 l m} \sum_{i_{1}=1}^{\infty} \sum_{j_{1}=0}^{\infty} \sum_{k_{1}=0}^{\infty} \sum_{l_{1}=0}^{\infty} \sum_{m_{1}=0}^{\infty} i_{1} P_{i_{1} j_{1} k_{1} l_{1} m_{1}} \\
& +k_{\operatorname{trp}} \sum_{i=1}^{\infty} \sum_{j=1}^{\infty} \sum_{k=1}^{\infty} \sum_{l=0}^{\infty} \sum_{m=0}^{\infty} i k P_{i j-1 k-1 l m} \sum_{i_{1}=1}^{\infty} \sum_{j_{1}=0}^{\infty} \sum_{k_{1}=0}^{\infty} \sum_{l_{1}=0}^{\infty} \sum_{m_{1}=0}^{\infty} k_{1} P_{i_{1} j_{1} k_{1} l_{1} m_{1}}
\end{aligned}
$$




$$
\begin{aligned}
& -k_{\operatorname{trp}} \sum_{i=1}^{\infty} \sum_{j=0}^{\infty} \sum_{k=0}^{\infty} \sum_{l=0}^{\infty} \sum_{m=0}^{\infty} k m P_{i j k l m} \sum_{i_{1}=1}^{\infty} \sum_{j_{1}=0}^{\infty} \sum_{k_{1}=0}^{\infty} \sum_{l_{1}=0}^{\infty} \sum_{m_{1}=0}^{\infty} i_{1} P_{i_{1} j_{1} k_{1} l_{1} m_{1}} \\
& -k_{\operatorname{trp}} \sum_{i=1}^{\infty} \sum_{j=0}^{\infty} \sum_{k=0}^{\infty} \sum_{l=0}^{\infty} \sum_{m=0}^{\infty} i k P_{i j k l m} \sum_{i_{1}=1}^{\infty} \sum_{j_{1}=0}^{\infty} \sum_{k_{1}=0}^{\infty} \sum_{l_{1}=0}^{\infty} \sum_{m_{1}=0}^{\infty} m_{1} P_{i_{1} j_{1} k_{1} l_{1} m_{1}} \\
& +k_{\operatorname{trp}} \sum_{i=1}^{\infty} \sum_{j=0}^{\infty} \sum_{k=0}^{\infty} \sum_{l=0}^{\infty} \sum_{m=0}^{\infty} k(m+1) P_{i j k l m+1} \sum_{i_{1}=1}^{\infty} \sum_{j_{1}=0}^{\infty} \sum_{k_{1}=0}^{\infty} \sum_{l_{1}=0}^{\infty} \sum_{m_{1}=0}^{\infty} i_{1} P_{i_{1} j_{1} k_{1} l_{1} m_{1}} \\
& +k_{\text {trp }} \sum_{i=1}^{\infty} \sum_{j=1}^{\infty} \sum_{k=0}^{\infty} \sum_{l=0}^{\infty} \sum_{m=1}^{\infty} i k P_{i j-1 k l m-1} \sum_{i_{1}=1}^{\infty} \sum_{j_{1}=0}^{\infty} \sum_{k_{1}=0}^{\infty} \sum_{l_{1}=0}^{\infty} \sum_{m_{1}=0}^{\infty} m_{1} P_{i_{1} j_{1} k_{1} l_{1} m_{1}} \\
& -k_{i n t r} \sum_{i=1}^{\infty} \sum_{j=0}^{\infty} \sum_{k=0}^{\infty} \sum_{l=0}^{\infty} \sum_{m=0}^{\infty} k^{2} P_{i j k l m} \\
& +k_{i n t r} \sum_{i=1}^{\infty} \sum_{j=1}^{\infty} \sum_{k=0}^{\infty} \sum_{l=0}^{\infty} \sum_{m=0}^{\infty} k^{2} P_{i j-1 k l m} \\
& -k_{r} \sum_{i=1}^{\infty} \sum_{j=0}^{\infty} \sum_{k=0}^{\infty} \sum_{l=0}^{\infty} \sum_{m=0}^{\infty} k m P_{i j k l m} \\
& +k_{r} \sum_{i=1}^{\infty} \sum_{j=0}^{\infty} \sum_{k=1}^{\infty} \sum_{l=0}^{\infty} \sum_{m=0}^{\infty} k(m+1) P_{i j k-1 l m+1} \\
& -k_{t c} \sum_{i=1}^{\infty} \sum_{j=0}^{\infty} \sum_{k=0}^{\infty} \sum_{l=0}^{\infty} \sum_{m=0}^{\infty} k^{2} P_{i j k l m} \sum_{i_{1}=1}^{\infty} \sum_{j_{1}=0}^{\infty} \sum_{k_{1}=0}^{\infty} \sum_{l_{1}=0}^{\infty} \sum_{m_{1}=0}^{\infty} k_{1} P_{i_{1} j_{1} k_{1} l_{1} m_{1}} \\
& +\frac{1}{2} k_{t c} \sum_{i=1}^{\infty} \sum_{j=0}^{\infty} \sum_{k=0}^{\infty} \sum_{l=0}^{\infty} \sum_{m=0}^{\infty} \sum_{i_{1}=1}^{i} \sum_{j_{1}=0}^{j} \sum_{k_{1}=0}^{k+2} \sum_{l_{1}=0}^{l} \sum_{m_{1}=0}^{m} k\left[k_{1} P_{i_{1} j_{1} k_{1} l_{1} m_{1}}\left(k-k_{1}+2\right) P_{i-i_{1}, j-j_{1}, k-k_{1}+2, l-l_{1} m-m_{1}}\right] \\
& -k_{t c} \sum_{i=1}^{\infty} \sum_{j=0}^{\infty} \sum_{k=0}^{\infty} \sum_{l=0}^{\infty} \sum_{m=0}^{\infty} k m P_{i j k l m} \sum_{i_{1}=1}^{\infty} \sum_{j_{1}=0}^{\infty} \sum_{k_{1}=0}^{\infty} \sum_{l_{1}=0}^{\infty} \sum_{m_{1}=0}^{\infty} m_{1} P_{i_{1} j_{1} k_{1} l_{1} m_{1}} \\
& +\frac{1}{2} k_{t c} \sum_{i=1}^{\infty} \sum_{j=0}^{\infty} \sum_{k=0}^{\infty} \sum_{l=0}^{\infty} \sum_{m=0}^{\infty} \sum_{i_{1}=1}^{i} \sum_{j_{1}=0}^{j} \sum_{k_{1}=0}^{k} \sum_{l_{1}=0}^{l} \sum_{m_{1}=0}^{m+2}\left[k m_{1} P_{i_{1} j_{1} k_{1} l_{1} m_{1}}\left(m+2-m_{1}\right) P_{i-i_{1}, j-j_{1}, k-k_{1}, l-l_{1} m+2-m_{1}}\right] \\
& -k_{t c} \sum_{i=1}^{\infty} \sum_{j=0}^{\infty} \sum_{k=0}^{\infty} \sum_{l=0}^{\infty} \sum_{m=0}^{\infty} k^{2} P_{i j k l m} \sum_{i_{1}=1}^{\infty} \sum_{j_{1}=0}^{\infty} \sum_{k_{1}=0}^{\infty} \sum_{l_{1}=0}^{\infty} \sum_{m_{1}=0}^{\infty} m_{1} P_{i_{1} j_{1} k_{1} l_{1} m_{1}} \\
& -k_{t c} \sum_{i=1}^{\infty} \sum_{j=0}^{\infty} \sum_{k=0}^{\infty} \sum_{l=0}^{\infty} \sum_{m=0}^{\infty} k m P_{i j k l m} \sum_{i_{1}=1}^{\infty} \sum_{j_{1}=0}^{\infty} \sum_{k_{1}=0}^{\infty} \sum_{l_{1}=0}^{\infty} \sum_{m_{1}=0}^{\infty} k_{1} P_{i_{1} j_{1} k_{1} l_{1} m_{1}} \\
& +\frac{1}{2} k_{t c} \sum_{i=1}^{\infty} \sum_{j=0}^{\infty} \sum_{k=0}^{\infty} \sum_{l=0}^{\infty} \sum_{m=0}^{\infty} \sum_{i_{1}=1}^{i} \sum_{j_{1}=0}^{j} \sum_{k_{1}=0}^{k+1} \sum_{l_{1}=0}^{l} \sum_{m_{1}=0}^{m+1}\left[k k_{1} P_{i_{1} j_{1} k_{1} l_{1} m_{1}}\left(m+1-m_{1}\right) P_{\left.i-i_{1}, j-j_{1}, k+1-k_{1}, l-l_{1} m+1-m_{1}\right]}\right. \\
& +\frac{1}{2} k_{t c} \sum_{i=1}^{\infty} \sum_{j=0}^{\infty} \sum_{k=0}^{\infty} \sum_{l=0}^{\infty} \sum_{m=0}^{\infty} \sum_{i_{1}=1}^{i} \sum_{j_{1}=0}^{j} \sum_{k_{1}=0}^{k+1} \sum_{l_{1}=0}^{l} \sum_{m_{1}=0}^{m+1}\left[k m_{1} P_{i_{1} j_{1} k_{1} l_{1} m_{1}}\left(k+1-k_{1}\right) P_{i-i_{1}, j-j_{1}, k+1-k_{1}, l-l_{1} m+1-m_{1}}\right]
\end{aligned}
$$


Now,

$$
\begin{array}{rl}
\sum_{k=0}^{\infty} \sum_{k_{1}=0}^{k+2} & k k_{1} P_{k_{1}}\left(k+2-k_{1}\right) P_{k+2-k_{1}} \\
& =\sum_{k=0}^{\infty} \sum_{k_{1}=0}^{k+2}\left[k_{1}+\left(k-k_{1}\right)\right] k_{1} P_{k_{1}}\left(k+2-k_{1}\right) P_{k+2-k_{1}} \\
& =\sum_{a=0}^{1}\left(\begin{array}{l}
1 \\
a
\end{array}\right) \sum_{k=0}^{\infty} \sum_{k_{1}=0}^{k+2} k_{1}^{1+a} P_{k_{1}}\left(k+2-k_{1}-2\right)^{1-a}\left(k+2-k_{1}\right) P_{k+2-k_{1}} \\
& =\sum_{a=0}^{1}\left(\begin{array}{l}
1 \\
a
\end{array}\right) \sum_{k=0}^{\infty} k^{1+a} P_{k} \sum_{k_{1}=0}^{\infty}\left(k_{1}-2\right)^{1-a} k_{1} P_{k_{1}} \\
& =\sum_{a=0}^{1}\left(\begin{array}{l}
1 \\
a
\end{array}\right) \mu_{1+a} \sum_{k_{1}=0}^{\infty}\left(k_{1}-2\right)^{1-a} k_{1} P_{k_{1}} \\
& =\left(\frac{1 !}{0 ! 1 !} \mu_{1} \sum_{k_{1}=0}^{\infty}\left(k_{1}-2\right) k_{1} P_{k_{1}}\right)+\left(\frac{1 !}{1 ! 0 !} \mu_{2} \sum_{k_{1}=0}^{\infty} k_{1} P_{k_{1}}\right) \\
& =\mu_{1} \mu_{2}-2 \mu_{1}^{2}+\mu_{2} \mu_{1} \\
= & 2 \mu_{2} \mu_{1}-2 \mu_{1}^{2}
\end{array}
$$

Similarly,

$$
\begin{aligned}
\sum_{k=0}^{\infty} \sum_{k_{1}=0}^{k+1} & \sum_{m=0}^{\infty} \sum_{m_{1}=0}^{m+1} k k_{1} P_{k_{1}, m_{1}}\left(m+1-m_{1}\right) P_{k+1-k_{1}, m+1-m_{1}} \\
= & \sum_{k=0}^{\infty} \sum_{k_{1}=0}^{k+1} \sum_{m=0}^{\infty} \sum_{m_{1}=0}^{m+1}\left[k_{1}+\left(k-k_{1}\right)\right] k_{1} P_{k_{1}, m_{1}}\left(m+1-m_{1}\right) P_{k+1-k_{1}, m+1-m_{1}} \\
= & \sum_{a=0}^{1}\left(\begin{array}{l}
1 \\
a
\end{array}\right) \sum_{k=0}^{\infty} \sum_{k_{1}=0}^{k+1} \sum_{m=0}^{\infty} \sum_{m_{1}=0}^{m+1} k_{1}^{1+a} P_{k_{1}, m_{1}}\left(k+1-k_{1}-1\right)^{1-a}\left(m+1-m_{1}\right) P_{k+1-k_{1}, m+1-m_{1}} \\
= & \sum_{a=0}^{1}\left(\begin{array}{l}
1 \\
a
\end{array}\right) \sum_{k=0}^{\infty} \sum_{m=0}^{\infty} k^{1+a} P_{k, m} \sum_{k_{1}=0}^{\infty} \sum_{m_{1}=0}^{\infty}\left(k_{1}-1\right)^{1-a} m_{1} P_{k_{1}, m_{1}} \\
= & \sum_{a=0}^{1}\left(\begin{array}{l}
1 \\
a
\end{array}\right) \mu_{1+a, 0} \sum_{k_{1}=0}^{\infty} \sum_{m_{1}=0}^{\infty}\left(k_{1}-1\right)^{1-a} m_{1} P_{k_{1}, m_{1}} \\
= & \left(\frac{1 !}{0 ! 1 !} \mu_{10} \sum_{k_{1}=0}^{\infty} \sum_{m_{1}=0}^{\infty}\left(k_{1}-1\right) m_{1} P_{k_{1}, m_{1}}\right)+\left(\frac{1 !}{1 ! 0 !} \mu_{20} \sum_{k_{1}=0}^{\infty} \sum_{m_{1}=0}^{\infty} m_{1} P_{k_{1}, m_{1}}\right) \\
= & \mu_{10} \mu_{11}-\mu_{10} \mu_{01}+\mu_{20} \mu_{01}
\end{aligned}
$$


and

$$
\begin{aligned}
\sum_{k=0}^{\infty} \sum_{k_{1}=0}^{k+1} & \sum_{m=0}^{\infty} \sum_{m_{1}=0}^{m+1} k m_{1} P_{k_{1}, m_{1}}\left(k+1-k_{1}\right) P_{k+1-k_{1}, m+1-m_{1}} \\
= & \sum_{k=0}^{\infty} \sum_{k_{1}=0}^{k+1} \sum_{m=0}^{\infty} \sum_{m_{1}=0}^{m+1}\left[k_{1}+\left(k-k_{1}\right)\right] m_{1} P_{k_{1}, m_{1}}\left(k+1-k_{1}\right) P_{k+1-k_{1}, m+1-m_{1}} \\
= & \sum_{a=0}^{1}\left(\begin{array}{l}
1 \\
a
\end{array}\right) \sum_{k=0}^{\infty} \sum_{k_{1}=0}^{k+1} \sum_{m=0}^{\infty} \sum_{m_{1}=0}^{m+1} k_{1}^{a} m_{1} P_{k_{1}, m_{1}}\left(k+1-k_{1}-1\right)^{1-a}\left(k+1-k_{1}\right) P_{k+1-k_{1}, m+1-m_{1}} \\
= & \sum_{a=0}^{1}\left(\begin{array}{l}
1 \\
a
\end{array}\right) \sum_{k=0}^{\infty} \sum_{m=0}^{\infty} k^{a} m_{1} P_{k, m} \sum_{k_{1}=0}^{\infty} \sum_{m_{1}=0}^{\infty}\left(k_{1}-1\right)^{1-a} k_{1} P_{k_{1}, m_{1}} \\
= & \sum_{a=0}^{1}\left(\begin{array}{l}
1 \\
a
\end{array}\right) \mu_{a, 1} \sum_{k_{1}=0}^{\infty} \sum_{m_{1}=0}^{\infty}\left(k_{1}-1\right)^{1-a} k_{1} P_{k_{1}, m_{1}} \\
= & \left(\frac{1 !}{0 ! 1 !} \mu_{01} \sum_{k_{1}=0}^{\infty} \sum_{m_{1}=0}^{\infty}\left(k_{1}-1\right) k_{1} P_{k_{1}, m_{1}}\right)+\left(\frac{1 !}{1 ! 0 !} \mu_{11} \sum_{k_{1}=0}^{\infty} \sum_{m_{1}=0}^{\infty} k_{1} P_{k_{1}, m_{1}}\right) \\
= & \mu_{20} \mu_{01}-\mu_{10} \mu_{01}+\mu_{11} \mu_{10}
\end{aligned}
$$

$$
\begin{aligned}
& \Rightarrow \frac{d \mu_{00100}}{d t}=k_{i} C_{M} C_{R} \\
& -k_{p} C_{M} \sum_{i=1}^{\infty} \sum_{j=0}^{\infty} \sum_{k=0}^{\infty} \sum_{l=0}^{\infty} \sum_{m=0}^{\infty} k^{2} P_{i j k l m} \\
& +k_{p} C_{M} \sum_{i=1}^{\infty} \sum_{j=0}^{\infty} \sum_{k=0}^{\infty} \sum_{l=0}^{\infty} \sum_{m=0}^{\infty} k^{2} P_{i j k l m} \\
& -k_{p} C_{M} \sum_{i=1}^{\infty} \sum_{j=0}^{\infty} \sum_{k=0}^{\infty} \sum_{l=0}^{\infty} \sum_{m=0}^{\infty} k m P_{i j k l m} \\
& +k_{p} C_{M} \sum_{i=1}^{\infty} \sum_{j=0}^{\infty} \sum_{k=0}^{\infty} \sum_{l=0}^{\infty} \sum_{m=0}^{\infty} k m P_{i j k l m} \\
& -k_{t r t} C_{T} \sum_{i=1}^{\infty} \sum_{j=0}^{\infty} \sum_{k=0}^{\infty} \sum_{l=0}^{\infty} \sum_{m=0}^{\infty} k^{2} P_{i j k l m} \\
& +k_{t r t} C_{T} \sum_{i=1}^{\infty} \sum_{j=0}^{\infty} \sum_{k=0}^{\infty} \sum_{l=0}^{\infty} \sum_{m=0}^{\infty}(k-1) k P_{i j k l m}
\end{aligned}
$$




$$
\begin{aligned}
& -k_{t r t} \sum_{i=1}^{\infty} \sum_{j=0}^{\infty} \sum_{k=0}^{\infty} \sum_{l=0}^{\infty} \sum_{m=0}^{\infty} k^{2} P_{i j k l m} \sum_{i_{1}=1}^{\infty} \sum_{j_{1}=0}^{\infty} \sum_{k_{1}=0}^{\infty} \sum_{l_{1}=0}^{\infty} \sum_{m_{1}=0}^{\infty} l_{1} P_{i_{1} j_{1} k_{1} l_{1} m_{1}} \\
& -k_{t r t} \sum_{i=1}^{\infty} \sum_{j=0}^{\infty} \sum_{k=0}^{\infty} \sum_{l=0}^{\infty} \sum_{m=0}^{\infty} k l P_{i j k l m} \sum_{i_{1}=1}^{\infty} \sum_{j_{1}=0}^{\infty} \sum_{k_{1}=0}^{\infty} \sum_{l_{1}=0}^{\infty} \sum_{m_{1}=0}^{\infty} k_{1} P_{i_{1} j_{1} k_{1} l_{1} m_{1}} \\
& +k_{t r t} \sum_{i=1}^{\infty} \sum_{j=0}^{\infty} \sum_{k=0}^{\infty} \sum_{l=0}^{\infty} \sum_{m=0}^{\infty}(k-1) k P_{i j k l m} \sum_{i_{1}=1}^{\infty} \sum_{j_{1}=0}^{\infty} \sum_{k_{1}=0}^{\infty} \sum_{l_{1}=0}^{\infty} \sum_{m_{1}=0}^{\infty} l_{1} P_{i_{1} j_{1} k_{1} l_{1} m_{1}} \\
& +k_{t r t} \sum_{i=1}^{\infty} \sum_{j=0}^{\infty} \sum_{k=0}^{\infty} \sum_{l=0}^{\infty} \sum_{m=0}^{\infty} k l P_{i j k l m} \sum_{i_{1}=1}^{\infty} \sum_{j_{1}=0}^{\infty} \sum_{k_{1}=0}^{\infty} \sum_{l_{1}=0}^{\infty} \sum_{m_{1}=0}^{\infty} k_{1} P_{i_{1} j_{1} k_{1} l_{1} m_{1}} \\
& -k_{t r p} \sum_{i=1}^{\infty} \sum_{j=0}^{\infty} \sum_{k=0}^{\infty} \sum_{l=0}^{\infty} \sum_{m=0}^{\infty} k^{2} P_{i j k l m} \sum_{i_{1}=1}^{\infty} \sum_{j_{1}=0}^{\infty} \sum_{k_{1}=0}^{\infty} \sum_{l_{1}=0}^{\infty} \sum_{m_{1}=0}^{\infty} i_{1} P_{i_{1} j_{1} k_{1} l_{1} m_{1}} \\
& -k_{t r p} \sum_{i=1}^{\infty} \sum_{j=0}^{\infty} \sum_{k=0}^{\infty} \sum_{l=0}^{\infty} \sum_{m=0}^{\infty} i k P_{i j k l m} \sum_{i_{1}=1}^{\infty} \sum_{j_{1}=0}^{\infty} \sum_{k_{1}=0}^{\infty} \sum_{l_{1}=0}^{\infty} \sum_{m_{1}=0}^{\infty} k_{1} P_{i_{1} j_{1} k_{1} l_{1} m_{1}} \\
& +k_{\operatorname{trp}} \sum_{i=1}^{\infty} \sum_{j=0}^{\infty} \sum_{k=0}^{\infty} \sum_{l=0}^{\infty} \sum_{m=0}^{\infty}(k-1) k P_{i j k l m} \sum_{i_{1}=1}^{\infty} \sum_{j_{1}=0}^{\infty} \sum_{k_{1}=0}^{\infty} \sum_{l_{1}=0}^{\infty} \sum_{m_{1}=0}^{\infty} i_{1} P_{i_{1} j_{1} k_{1} l_{1} m_{1}} \\
& +k_{t r p} \sum_{i=1}^{\infty} \sum_{j=0}^{\infty} \sum_{k=0}^{\infty} \sum_{l=0}^{\infty} \sum_{m=0}^{\infty} i(k+1) P_{i j k l m} \sum_{i_{1}=1}^{\infty} \sum_{j_{1}=0}^{\infty} \sum_{k_{1}=0}^{\infty} \sum_{l_{1}=0}^{\infty} \sum_{m_{1}=0}^{\infty} k_{1} P_{i_{1} j_{1} k_{1} l_{1} m_{1}} \\
& -k_{\operatorname{trp}} \sum_{i=1}^{\infty} \sum_{j=0}^{\infty} \sum_{k=0}^{\infty} \sum_{l=0}^{\infty} \sum_{m=0}^{\infty} k m P_{i j k l m} \sum_{i_{1}=1}^{\infty} \sum_{j_{1}=0}^{\infty} \sum_{k_{1}=0}^{\infty} \sum_{l_{1}=0}^{\infty} \sum_{m_{1}=0}^{\infty} i_{1} P_{i_{1} j_{1} k_{1} l_{1} m_{1}} \\
& -k_{\operatorname{trp}} \sum_{i=1}^{\infty} \sum_{j=0}^{\infty} \sum_{k=0}^{\infty} \sum_{l=0}^{\infty} \sum_{m=0}^{\infty} i k P_{i j k l m} \sum_{i_{1}=1}^{\infty} \sum_{j_{1}=0}^{\infty} \sum_{k_{1}=0}^{\infty} \sum_{l_{1}=0}^{\infty} \sum_{m_{1}=0}^{\infty} m_{1} P_{i_{1} j_{1} k_{1} l_{1} m_{1}} \\
& +k_{\operatorname{trp}} \sum_{i=1}^{\infty} \sum_{j=0}^{\infty} \sum_{k=0}^{\infty} \sum_{l=0}^{\infty} \sum_{m=0}^{\infty} k m P_{i j k l m} \sum_{i_{1}=1}^{\infty} \sum_{j_{1}=0}^{\infty} \sum_{k_{1}=0}^{\infty} \sum_{l_{1}=0}^{\infty} \sum_{m_{1}=0}^{\infty} i_{1} P_{i_{1} j_{1} k_{1} l_{1} m_{1}} \\
& +k_{\text {trp }} \sum_{i=1}^{\infty} \sum_{j=0}^{\infty} \sum_{k=0}^{\infty} \sum_{l=0}^{\infty} \sum_{m=0}^{\infty} i k P_{i j k l m} \sum_{i_{1}=1}^{\infty} \sum_{j_{1}=0}^{\infty} \sum_{k_{1}=0}^{\infty} \sum_{l_{1}=0}^{\infty} \sum_{m_{1}=0}^{\infty} m_{1} P_{i_{1} j_{1} k_{1} l_{1} m_{1}} \\
& -k_{i n t r} \sum_{i=1}^{\infty} \sum_{j=0}^{\infty} \sum_{k=0}^{\infty} \sum_{l=0}^{\infty} \sum_{m=0}^{\infty} k^{2} P_{i j k l m} \\
& +k_{i n t r} \sum_{i=1}^{\infty} \sum_{j=0}^{\infty} \sum_{k=0}^{\infty} \sum_{l=0}^{\infty} \sum_{m=0}^{\infty} k^{2} P_{i j k l m} \\
& -k_{r} \sum_{i=1}^{\infty} \sum_{j=0}^{\infty} \sum_{k=0}^{\infty} \sum_{l=0}^{\infty} \sum_{m=0}^{\infty} k m P_{i j k l m} \\
& +k_{r} \sum_{i=1}^{\infty} \sum_{j=0}^{\infty} \sum_{k=0}^{\infty} \sum_{l=0}^{\infty} \sum_{m=0}^{\infty}(k+1) m P_{i j k l m}
\end{aligned}
$$




$$
\begin{aligned}
& -k_{t c} \sum_{i=1}^{\infty} \sum_{j=0}^{\infty} \sum_{k=0}^{\infty} \sum_{l=0}^{\infty} \sum_{m=0}^{\infty} k^{2} P_{i j k l m} \sum_{i_{1}=1}^{\infty} \sum_{j_{1}=0}^{\infty} \sum_{k_{1}=0}^{\infty} \sum_{l_{1}=0}^{\infty} \sum_{m_{1}=0}^{\infty} k_{1} P_{i_{1} j_{1} k_{1} l_{1} m_{1}} \\
& +\frac{1}{2}(2) k_{t c} \sum_{i=1}^{\infty} \sum_{j=0}^{\infty} \sum_{k=0}^{\infty} \sum_{l=0}^{\infty} \sum_{m=0}^{\infty} k^{2} P_{i j k l m} \sum_{i_{1}=1}^{\infty} \sum_{j_{1}=0}^{\infty} \sum_{k_{1}=0}^{\infty} \sum_{l_{1}=0}^{\infty} \sum_{m_{1}=0}^{\infty} k_{1} P_{i_{1} j_{1} k_{1} l_{1} m_{1}} \\
& -\frac{1}{2}(2) k_{t c} \sum_{i=1}^{\infty} \sum_{j=0}^{\infty} \sum_{k=0}^{\infty} \sum_{l=0}^{\infty} \sum_{m=0}^{\infty} k P_{i j k l m} \sum_{i_{1}=1}^{\infty} \sum_{j_{1}=0}^{\infty} \sum_{k_{1}=0}^{\infty} \sum_{l_{1}=0}^{\infty} \sum_{m_{1}=0}^{\infty} k_{1} P_{i_{1} j_{1} k_{1} l_{1} m_{1}} \\
& -k_{t c} \sum_{i=1}^{\infty} \sum_{j=0}^{\infty} \sum_{k=0}^{\infty} \sum_{l=0}^{\infty} \sum_{m=0}^{\infty} k m P_{i j k l m} \sum_{i_{1}=1}^{\infty} \sum_{j_{1}=0}^{\infty} \sum_{k_{1}=0}^{\infty} \sum_{l_{1}=0}^{\infty} \sum_{m_{1}=0}^{\infty} m_{1} P_{i_{1} j_{1} k_{1} l_{1} m_{1}} \\
& +\frac{1}{2}(2) k_{t c} \sum_{i=1}^{\infty} \sum_{j=0}^{\infty} \sum_{k=0}^{\infty} \sum_{l=0}^{\infty} \sum_{m=0}^{\infty} k m P_{i j k l m} \sum_{i_{1}=1}^{\infty} \sum_{j_{1}=0}^{\infty} \sum_{k_{1}=0}^{\infty} \sum_{l_{1}=0}^{\infty} \sum_{m_{1}=0}^{\infty} m_{1} P_{i_{1} j_{1} k_{1} l_{1} m_{1}} \\
& -k_{t c} \sum_{i=1}^{\infty} \sum_{j=0}^{\infty} \sum_{k=0}^{\infty} \sum_{l=0}^{\infty} \sum_{m=0}^{\infty} k^{2} P_{i j k l m} \sum_{i_{1}=1}^{\infty} \sum_{j_{1}=0}^{\infty} \sum_{k_{1}=0}^{\infty} \sum_{l_{1}=0}^{\infty} \sum_{m_{1}=0}^{\infty} m_{1} P_{i_{1} j_{1} k_{1} l_{1} m_{1}} \\
& -k_{t c} \sum_{i=1}^{\infty} \sum_{j=0}^{\infty} \sum_{k=0}^{\infty} \sum_{l=0}^{\infty} \sum_{m=0}^{\infty} k m P_{i j k l m} \sum_{i_{1}=1}^{\infty} \sum_{j_{1}=0}^{\infty} \sum_{k_{1}=0}^{\infty} \sum_{l_{1}=0}^{\infty} \sum_{m_{1}=0}^{\infty} k_{1} P_{i_{1} j_{1} k_{1} l_{1} m_{1}} \\
& +\frac{1}{2}(2) k_{t c} \sum_{i=1}^{\infty} \sum_{j=0}^{\infty} \sum_{k=0}^{\infty} \sum_{l=0}^{\infty} \sum_{m=0}^{\infty} k^{2} P_{i j k l m} \sum_{i_{1}=1}^{\infty} \sum_{j_{1}=0}^{\infty} \sum_{k_{1}=0}^{\infty} \sum_{l_{1}=0}^{\infty} \sum_{m_{1}=0}^{\infty} m_{1} P_{i_{1} j_{1} k_{1} l_{1} m_{1}} \\
& +\frac{1}{2}(2) k_{t c} \sum_{i=1}^{\infty} \sum_{j=0}^{\infty} \sum_{k=0}^{\infty} \sum_{l=0}^{\infty} \sum_{m=0}^{\infty} k m P_{i j k l m} \sum_{i_{1}=1}^{\infty} \sum_{j_{1}=0}^{\infty} \sum_{k_{1}=0}^{\infty} \sum_{l_{1}=0}^{\infty} \sum_{m_{1}=0}^{\infty} k_{1} P_{i_{1} j_{1} k_{1} l_{1} m_{1}} \\
& -\frac{1}{2}(2) k_{t c} \sum_{i=1}^{\infty} \sum_{j=0}^{\infty} \sum_{k=0}^{\infty} \sum_{l=0}^{\infty} \sum_{m=0}^{\infty} k P_{i j k l m} \sum_{i_{1}=1}^{\infty} \sum_{j_{1}=0}^{\infty} \sum_{k_{1}=0}^{\infty} \sum_{l_{1}=0}^{\infty} \sum_{m_{1}=0}^{\infty} m_{1} P_{i_{1} j_{1} k_{1} l_{1} m_{1}}
\end{aligned}
$$

using Equations (42), (52), (53) and (54).

$$
\begin{aligned}
& \Rightarrow \frac{d \mu_{00100}}{d t}=k_{i} C_{M} C_{R} \\
& \quad-k_{t r t} C_{T} \sum_{i=1}^{\infty} \sum_{j=0}^{\infty} \sum_{k=0}^{\infty} \sum_{l=0}^{\infty} \sum_{m=0}^{\infty} k P_{i j k l m} \\
& \quad-k_{t r t} \sum_{i=1}^{\infty} \sum_{j=0}^{\infty} \sum_{k=0}^{\infty} \sum_{l=0}^{\infty} \sum_{m=0}^{\infty} k P_{i j k l m} \sum_{i_{1}=1}^{\infty} \sum_{j_{1}=0}^{\infty} \sum_{k_{1}=0}^{\infty} \sum_{l_{1}=0}^{\infty} \sum_{m_{1}=0}^{\infty} l_{1} P_{i_{1} j_{1} k_{1} l_{1} m_{1}} \\
& \quad-k_{t r p} \sum_{i=1}^{\infty} \sum_{j=0}^{\infty} \sum_{k=0}^{\infty} \sum_{l=0}^{\infty} \sum_{m=0}^{\infty} k P_{i j k l m} \sum_{i_{1}=1}^{\infty} \sum_{j_{1}=0}^{\infty} \sum_{k_{1}=0}^{\infty} \sum_{l_{1}=0}^{\infty} \sum_{m_{1}=0}^{\infty} i_{1} P_{i_{1} j_{1} k_{1} l_{1} m_{1}} \\
& \quad+k_{t r p} \sum_{i=1}^{\infty} \sum_{j=0}^{\infty} \sum_{k=0}^{\infty} \sum_{l=0}^{\infty} \sum_{m=0}^{\infty} i P_{i j k l m} \sum_{i_{1}=1}^{\infty} \sum_{j_{1}=0}^{\infty} \sum_{k_{1}=0}^{\infty} \sum_{l_{1}=0}^{\infty} \sum_{m_{1}=0}^{\infty} k_{1} P_{i_{1} j_{1} k_{1} l_{1} m_{1}} \\
& \quad+k_{r} \sum_{i=1}^{\infty} \sum_{j=0}^{\infty} \sum_{k=0}^{\infty} \sum_{l=0}^{\infty} \sum_{m=0}^{\infty} m P_{i j k l m} \\
& \quad-k_{t c} \sum_{i=1}^{\infty} \sum_{j=0}^{\infty} \sum_{k=0}^{\infty} \sum_{l=0}^{\infty} \sum_{m=0}^{\infty} k P_{i j k l m} \sum_{i_{1}=1}^{\infty} \sum_{j_{1}=0}^{\infty} \sum_{k_{1}=0}^{\infty} \sum_{l_{1}=0}^{\infty} \sum_{m_{1}=0}^{\infty} k_{1} P_{i_{1} j_{1}} k_{1} l_{1} m_{1} \\
& \\
& \quad-k_{t c} \sum_{i=1}^{\infty} \sum_{j=0}^{\infty} \sum_{k=0}^{\infty} \sum_{l=0}^{\infty} \sum_{m=0}^{\infty} k P_{i j k l m} \sum_{i_{1}=1}^{\infty} \sum_{j_{1}=0}^{\infty} \sum_{k_{1}=0}^{\infty} \sum_{l_{1}=0}^{\infty} \sum_{m_{1}=0}^{\infty} m_{1} P_{i_{1} j_{1} k_{1} l_{1} m_{1}}
\end{aligned}
$$




$$
\begin{aligned}
& \Rightarrow \frac{d \mu_{00100}}{d t}=k_{i} C_{M} C_{R}-k_{t r t} C_{T} \mu_{00100}-k_{t r t} \mu_{00100} \mu_{00010}+k_{r} \mu_{00001} \\
& \quad-k_{t c}\left(\mu_{00100}\right)^{2}-k_{t c} \mu_{00100} \mu_{00001}
\end{aligned}
$$


3.2.4 First moment w.r.t. the number of inactive (capped) radicals

$$
\begin{aligned}
& \frac{d \mu_{00010}}{d t}=\sum_{i=1}^{\infty} \sum_{j=0}^{\infty} \sum_{k=0}^{\infty} \sum_{l=0}^{\infty} \sum_{m=0}^{\infty} l \frac{d P_{i j k l m}}{d t} \\
& =-k_{p} C_{M} \sum_{i=1}^{\infty} \sum_{j=0}^{\infty} \sum_{k=0}^{\infty} \sum_{l=0}^{\infty} \sum_{m=0}^{\infty} k l P_{i j k l m} \\
& +k_{p} C_{M} \sum_{i=2}^{\infty} \sum_{j=0}^{\infty} \sum_{k=0}^{\infty} \sum_{l=0}^{\infty} \sum_{m=0}^{\infty} k l P_{i-1 j k l m} \\
& -k_{p} C_{M} \sum_{i=1}^{\infty} \sum_{j=0}^{\infty} \sum_{k=0}^{\infty} \sum_{l=0}^{\infty} \sum_{m=0}^{\infty} l m P_{i j k l m} \\
& +k_{p} C_{M} \sum_{i=2}^{\infty} \sum_{j=0}^{\infty} \sum_{k=0}^{\infty} \sum_{l=0}^{\infty} \sum_{m=0}^{\infty} \operatorname{lm} P_{i-1 j k l m} \\
& -k_{t r t} C_{T} \sum_{i=1}^{\infty} \sum_{j=0}^{\infty} \sum_{k=0}^{\infty} \sum_{l=0}^{\infty} \sum_{m=0}^{\infty} k l P_{i j k l m} \\
& +k_{t r t} C_{T} \sum_{i=1}^{\infty} \sum_{j=0}^{\infty} \sum_{k=0}^{\infty} \sum_{l=1}^{\infty} \sum_{m=0}^{\infty}(k+1) l P_{i j k+1 l-1 m} \\
& -k_{t r t} \sum_{i=1}^{\infty} \sum_{j=0}^{\infty} \sum_{k=0}^{\infty} \sum_{l=0}^{\infty} \sum_{m=0}^{\infty} k l P_{i j k l m} \sum_{i_{1}=1}^{\infty} \sum_{j_{1}=0}^{\infty} \sum_{k_{1}=0}^{\infty} \sum_{l_{1}=0}^{\infty} \sum_{m_{1}=0}^{\infty} l_{1} P_{i_{1} j_{1} k_{1} l_{1} m_{1}} \\
& -k_{t r t} \sum_{i=1}^{\infty} \sum_{j=0}^{\infty} \sum_{k=0}^{\infty} \sum_{l=0}^{\infty} \sum_{m=0}^{\infty} l^{2} P_{i j k l m} \sum_{i_{1}=1}^{\infty} \sum_{j_{1}=0}^{\infty} \sum_{k_{1}=0}^{\infty} \sum_{l_{1}=0}^{\infty} \sum_{m_{1}=0}^{\infty} k_{1} P_{i_{1} j_{1} k_{1} l_{1} m_{1}} \\
& +k_{t r t} \sum_{i=1}^{\infty} \sum_{j=0}^{\infty} \sum_{k=0}^{\infty} \sum_{l=1}^{\infty} \sum_{m=0}^{\infty}(k+1) l P_{i j k+1 l-1 m} \sum_{i_{1}=1}^{\infty} \sum_{j_{1}=0}^{\infty} \sum_{k_{1}=0}^{\infty} \sum_{l_{1}=0}^{\infty} \sum_{m_{1}=0}^{\infty} l_{1} P_{i_{1} j_{1} k_{1} l_{1} m_{1}} \\
& +k_{t r t} \sum_{i=1}^{\infty} \sum_{j=0}^{\infty} \sum_{k=0}^{\infty} \sum_{l=0}^{\infty} \sum_{m=1}^{\infty} l(l+1) P_{i j k l+1 m-1} \sum_{i_{1}=1}^{\infty} \sum_{j_{1}=0}^{\infty} \sum_{k_{1}=0}^{\infty} \sum_{l_{1}=0}^{\infty} \sum_{m_{1}=0}^{\infty} k_{1} P_{i_{1} j_{1} k_{1} l_{1} m_{1}} \\
& -k_{t r p} \sum_{i=1}^{\infty} \sum_{j=0}^{\infty} \sum_{k=0}^{\infty} \sum_{l=0}^{\infty} \sum_{m=0}^{\infty} k l P_{i j k l m} \sum_{i_{1}=1}^{\infty} \sum_{j_{1}=0}^{\infty} \sum_{k_{1}=0}^{\infty} \sum_{l_{1}=0}^{\infty} \sum_{m_{1}=0}^{\infty} i_{1} P_{i_{1} j_{1} k_{1} l_{1} m_{1}} \\
& -k_{t r p} \sum_{i=1}^{\infty} \sum_{j=0}^{\infty} \sum_{k=0}^{\infty} \sum_{l=0}^{\infty} \sum_{m=0}^{\infty} i l P_{i j k l m} \sum_{i_{1}=1}^{\infty} \sum_{j_{1}=0}^{\infty} \sum_{k_{1}=0}^{\infty} \sum_{l_{1}=0}^{\infty} \sum_{m_{1}=0}^{\infty} k_{1} P_{i_{1} j_{1} k_{1} l_{1} m_{1}} \\
& +k_{t r p} \sum_{i=1}^{\infty} \sum_{j=0}^{\infty} \sum_{k=0}^{\infty} \sum_{l=0}^{\infty} \sum_{m=0}^{\infty}(k+1) l P_{i j k+1 l m} \sum_{i_{1}=1}^{\infty} \sum_{j_{1}=0}^{\infty} \sum_{k_{1}=0}^{\infty} \sum_{l_{1}=0}^{\infty} \sum_{m_{1}=0}^{\infty} i_{1} P_{i_{1} j_{1} k_{1} l_{1} m_{1}} \\
& +k_{\operatorname{trp}} \sum_{i=1}^{\infty} \sum_{j=1}^{\infty} \sum_{k=1}^{\infty} \sum_{l=0}^{\infty} \sum_{m=0}^{\infty} i l P_{i j-1 k-1 l m} \sum_{i_{1}=1}^{\infty} \sum_{j_{1}=0}^{\infty} \sum_{k_{1}=0}^{\infty} \sum_{l_{1}=0}^{\infty} \sum_{m_{1}=0}^{\infty} k_{1} P_{i_{1} j_{1} k_{1} l_{1} m_{1}}
\end{aligned}
$$




$$
\begin{aligned}
& -k_{t r p} \sum_{i=1}^{\infty} \sum_{j=0}^{\infty} \sum_{k=0}^{\infty} \sum_{l=0}^{\infty} \sum_{m=0}^{\infty} \operatorname{lm} P_{i j k l m} \sum_{i_{1}=1}^{\infty} \sum_{j_{1}=0}^{\infty} \sum_{k_{1}=0}^{\infty} \sum_{l_{1}=0}^{\infty} \sum_{m_{1}=0}^{\infty} i_{1} P_{i_{1} j_{1} k_{1} l_{1} m_{1}} \\
& -k_{t r p} \sum_{i=1}^{\infty} \sum_{j=0}^{\infty} \sum_{k=0}^{\infty} \sum_{l=0}^{\infty} \sum_{m=0}^{\infty} i l P_{i j k l m} \sum_{i_{1}=1}^{\infty} \sum_{j_{1}=0}^{\infty} \sum_{k_{1}=0}^{\infty} \sum_{l_{1}=0}^{\infty} \sum_{m_{1}=0}^{\infty} m_{1} P_{i_{1} j_{1} k_{1} l_{1} m_{1}} \\
& +k_{\operatorname{trp}} \sum_{i=1}^{\infty} \sum_{j=0}^{\infty} \sum_{k=0}^{\infty} \sum_{l=0}^{\infty} \sum_{m=0}^{\infty} l(m+1) P_{i j k l m+1} \sum_{i_{1}=1}^{\infty} \sum_{j_{1}=0}^{\infty} \sum_{k_{1}=0}^{\infty} \sum_{l_{1}=0}^{\infty} \sum_{m_{1}=0}^{\infty} i_{1} P_{i_{1} j_{1} k_{1} l_{1} m_{1}} \\
& +k_{t r p} \sum_{i=1}^{\infty} \sum_{j=1}^{\infty} \sum_{k=0}^{\infty} \sum_{l=0}^{\infty} \sum_{m=1}^{\infty} i l P_{i j-1 k l m-1} \sum_{i_{1}=1}^{\infty} \sum_{j_{1}=0}^{\infty} \sum_{k_{1}=0}^{\infty} \sum_{l_{1}=0}^{\infty} \sum_{m_{1}=0}^{\infty} m_{1} P_{i_{1} j_{1} k_{1} l_{1} m_{1}} \\
& -k_{i n t r} \sum_{i=1}^{\infty} \sum_{j=0}^{\infty} \sum_{k=0}^{\infty} \sum_{l=0}^{\infty} \sum_{m=0}^{\infty} k l P_{i j k l m} \\
& +k_{i n t r} \sum_{i=1}^{\infty} \sum_{j=1}^{\infty} \sum_{k=0}^{\infty} \sum_{l=0}^{\infty} \sum_{m=0}^{\infty} k l P_{i j-1 k l m} \\
& -k_{r} \sum_{i=1}^{\infty} \sum_{j=0}^{\infty} \sum_{k=0}^{\infty} \sum_{l=0}^{\infty} \sum_{m=0}^{\infty} \operatorname{lm} P_{i j k l m} \\
& +k_{r} \sum_{i=1}^{\infty} \sum_{j=0}^{\infty} \sum_{k=1}^{\infty} \sum_{l=0}^{\infty} \sum_{m=0}^{\infty} l(m+1) P_{i j k-1 l m+1} \\
& -k_{t c} \sum_{i=1}^{\infty} \sum_{j=0}^{\infty} \sum_{k=0}^{\infty} \sum_{l=0}^{\infty} \sum_{m=0}^{\infty} l k P_{i j k l m} \sum_{i_{1}=1}^{\infty} \sum_{j_{1}=0}^{\infty} \sum_{k_{1}=0}^{\infty} \sum_{l_{1}=0}^{\infty} \sum_{m_{1}=0}^{\infty} k_{1} P_{i_{1} j_{1} k_{1} l_{1} m_{1}} \\
& +\frac{1}{2} k_{t c} \sum_{i=1}^{\infty} \sum_{j=0}^{\infty} \sum_{k=0}^{\infty} \sum_{l=0}^{\infty} \sum_{m=0}^{\infty} \sum_{i_{1}=1}^{i} \sum_{j_{1}=0}^{j} \sum_{k_{1}=0}^{k+2} \sum_{l_{1}=0}^{l} \sum_{m_{1}=0}^{m} l\left[k_{1} P_{i_{1} j_{1} k_{1} l_{1} m_{1}}\left(k+2-k_{1}\right) P_{i-i_{1}, j-j_{1}, k+2-k_{1}, l-l_{1} m-m_{1}}\right] \\
& -k_{t c} \sum_{i=1}^{\infty} \sum_{j=0}^{\infty} \sum_{k=0}^{\infty} \sum_{l=0}^{\infty} \sum_{m=0}^{\infty} \operatorname{lm} P_{i j k l m} \sum_{i_{1}=1}^{\infty} \sum_{j_{1}=0}^{\infty} \sum_{k_{1}=0}^{\infty} \sum_{l_{1}=0}^{\infty} \sum_{m_{1}=0}^{\infty} m_{1} P_{i_{1} j_{1} k_{1} l_{1} m_{1}} \\
& +\frac{1}{2} k_{t c} \sum_{i=1}^{\infty} \sum_{j=0}^{\infty} \sum_{k=0}^{\infty} \sum_{l=0}^{\infty} \sum_{m=0}^{\infty} \sum_{i_{1}=1}^{i} \sum_{j_{1}=0}^{j} \sum_{k_{1}=0}^{k} \sum_{l_{1}=0}^{l} \sum_{m_{1}=0}^{m+2}\left[l m_{1} P_{i_{1} j_{1} k_{1} l_{1} m_{1}}\left(m+2-m_{1}\right) P_{i-i_{1}, j-j_{1}, k-k_{1}, l-l_{1} m+2-m_{1}}\right] \\
& -k_{t c} \sum_{i=1}^{\infty} \sum_{j=0}^{\infty} \sum_{k=0}^{\infty} \sum_{l=0}^{\infty} \sum_{m=0}^{\infty} k l P_{i j k l m} \sum_{i_{1}=1}^{\infty} \sum_{j_{1}=0}^{\infty} \sum_{k_{1}=0}^{\infty} \sum_{l_{1}=0}^{\infty} \sum_{m_{1}=0}^{\infty} m_{1} P_{i_{1} j_{1} k_{1} l_{1} m_{1}} \\
& -k_{t c} \sum_{i=1}^{\infty} \sum_{j=0}^{\infty} \sum_{k=0}^{\infty} \sum_{l=0}^{\infty} \sum_{m=0}^{\infty} \operatorname{lm} P_{i j k l m} \sum_{i_{1}=1}^{\infty} \sum_{j_{1}=0}^{\infty} \sum_{k_{1}=0}^{\infty} \sum_{l_{1}=0}^{\infty} \sum_{m_{1}=0}^{\infty} k_{1} P_{i_{1} j_{1} k_{1} l_{1} m_{1}} \\
& +\frac{1}{2} k_{t c} \sum_{i=1}^{\infty} \sum_{j=0}^{\infty} \sum_{k=0}^{\infty} \sum_{l=0}^{\infty} \sum_{m=0}^{\infty} \sum_{i_{1}=1}^{i} \sum_{j_{1}=0}^{j} \sum_{k_{1}=0}^{k+1} \sum_{l_{1}=0}^{l} \sum_{m_{1}=0}^{m+1}\left[l k_{1} P_{i_{1} j_{1} k_{1} l_{1} m_{1}}\left(m+1-m_{1}\right) P_{i-i_{1}, j-j_{1}, k+1-k_{1}, l-l_{1} m+1-m_{1}}\right] \\
& +\frac{1}{2} k_{t c} \sum_{i=1}^{\infty} \sum_{j=0}^{\infty} \sum_{k=0}^{\infty} \sum_{l=0}^{\infty} \sum_{m=0}^{\infty} \sum_{i_{1}=1}^{i} \sum_{j_{1}=0}^{j} \sum_{k_{1}=0}^{k+1} \sum_{l_{1}=0}^{l} \sum_{m_{1}=0}^{m+1}\left[l m_{1} P_{i_{1} j_{1} k_{1} l_{1} m_{1}}\left(k+1-k_{1}\right) P_{i-i_{1}, j-j_{1}, k+1-k_{1}, l-l_{1} m+1-m_{1}}\right]
\end{aligned}
$$




$$
\begin{aligned}
& \Rightarrow \frac{d \mu_{00010}}{d t}=-k_{p} C_{M} \sum_{i=1}^{\infty} \sum_{j=0}^{\infty} \sum_{k=0}^{\infty} \sum_{l=0}^{\infty} \sum_{m=0}^{\infty} k l P_{i j k l m} \\
& +k_{p} C_{M} \sum_{i=1}^{\infty} \sum_{j=0}^{\infty} \sum_{k=0}^{\infty} \sum_{l=0}^{\infty} \sum_{m=0}^{\infty} k l P_{i j k l m} \\
& -k_{p} C_{M} \sum_{i=1}^{\infty} \sum_{j=0}^{\infty} \sum_{k=0}^{\infty} \sum_{l=0}^{\infty} \sum_{m=0}^{\infty} l m P_{i j k l m} \\
& +k_{p} C_{M} \sum_{i=1}^{\infty} \sum_{j=0}^{\infty} \sum_{k=0}^{\infty} \sum_{l=0}^{\infty} \sum_{m=0}^{\infty} l m P_{i j k l m} \\
& -k_{t r t} C_{T} \sum_{i=1}^{\infty} \sum_{j=0}^{\infty} \sum_{k=0}^{\infty} \sum_{l=0}^{\infty} \sum_{m=0}^{\infty} k l P_{i j k l m} \\
& +k_{t r t} C_{T} \sum_{i=1}^{\infty} \sum_{j=0}^{\infty} \sum_{k=0}^{\infty} \sum_{l=0}^{\infty} \sum_{m=0}^{\infty} k(l+1) P_{i j k l m} \\
& -k_{t r t} \sum_{i=1}^{\infty} \sum_{j=0}^{\infty} \sum_{k=0}^{\infty} \sum_{l=0}^{\infty} \sum_{m=0}^{\infty} k l P_{i j k l m} \sum_{i_{1}=1}^{\infty} \sum_{j_{1}=0}^{\infty} \sum_{k_{1}=0}^{\infty} \sum_{l_{1}=0}^{\infty} \sum_{m_{1}=0}^{\infty} l_{1} P_{i_{1} j_{1} k_{1} l_{1} m_{1}} \\
& -k_{t r t} \sum_{i=1}^{\infty} \sum_{j=0}^{\infty} \sum_{k=0}^{\infty} \sum_{l=0}^{\infty} \sum_{m=0}^{\infty} l^{2} P_{i j k l m} \sum_{i_{1}=1}^{\infty} \sum_{j_{1}=0}^{\infty} \sum_{k_{1}=0}^{\infty} \sum_{l_{1}=0}^{\infty} \sum_{m_{1}=0}^{\infty} k_{1} P_{i_{1} j_{1} k_{1} l_{1} m_{1}} \\
& +k_{\text {trt }} \sum_{i=1}^{\infty} \sum_{j=0}^{\infty} \sum_{k=0}^{\infty} \sum_{l=0}^{\infty} \sum_{m=0}^{\infty} k(l+1) P_{i j k l m} \sum_{i_{1}=1}^{\infty} \sum_{j_{1}=0}^{\infty} \sum_{k_{1}=0}^{\infty} \sum_{l_{1}=0}^{\infty} \sum_{m_{1}=0}^{\infty} l_{1} P_{i_{1} j_{1} k_{1} l_{1} m_{1}} \\
& +k_{t r t} \sum_{i=1}^{\infty} \sum_{j=0}^{\infty} \sum_{k=0}^{\infty} \sum_{l=0}^{\infty} \sum_{m=0}^{\infty}(l-1) l P_{i j k l m} \sum_{i_{1}=1}^{\infty} \sum_{j_{1}=0}^{\infty} \sum_{k_{1}=0}^{\infty} \sum_{l_{1}=0}^{\infty} \sum_{m_{1}=0}^{\infty} k_{1} P_{i_{1} j_{1} k_{1} l_{1} m_{1}} \\
& -k_{t r p} \sum_{i=1}^{\infty} \sum_{j=0}^{\infty} \sum_{k=0}^{\infty} \sum_{l=0}^{\infty} \sum_{m=0}^{\infty} k l P_{i j k l m} \sum_{i_{1}=1}^{\infty} \sum_{j_{1}=0}^{\infty} \sum_{k_{1}=0}^{\infty} \sum_{l_{1}=0}^{\infty} \sum_{m_{1}=0}^{\infty} i_{1} P_{i_{1} j_{1} k_{1} l_{1} m_{1}} \\
& -k_{t r p} \sum_{i=1}^{\infty} \sum_{j=0}^{\infty} \sum_{k=0}^{\infty} \sum_{l=0}^{\infty} \sum_{m=0}^{\infty} i l P_{i j k l m} \sum_{i_{1}=1}^{\infty} \sum_{j_{1}=0}^{\infty} \sum_{k_{1}=0}^{\infty} \sum_{l_{1}=0}^{\infty} \sum_{m_{1}=0}^{\infty} k_{1} P_{i_{1} j_{1} k_{1} l_{1} m_{1}} \\
& +k_{t r p} \sum_{i=1}^{\infty} \sum_{j=0}^{\infty} \sum_{k=0}^{\infty} \sum_{l=0}^{\infty} \sum_{m=0}^{\infty} k l P_{i j k l m} \sum_{i_{1}=1}^{\infty} \sum_{j_{1}=0}^{\infty} \sum_{k_{1}=0}^{\infty} \sum_{l_{1}=0}^{\infty} \sum_{m_{1}=0}^{\infty} i_{1} P_{i_{1} j_{1} k_{1} l_{1} m_{1}} \\
& +k_{t r p} \sum_{i=1}^{\infty} \sum_{j=0}^{\infty} \sum_{k=0}^{\infty} \sum_{l=0}^{\infty} \sum_{m=0}^{\infty} i l P_{i j k l m} \sum_{i_{1}=1}^{\infty} \sum_{j_{1}=0}^{\infty} \sum_{k_{1}=0}^{\infty} \sum_{l_{1}=0}^{\infty} \sum_{m_{1}=0}^{\infty} k_{1} P_{i_{1} j_{1} k_{1} l_{1} m_{1}}
\end{aligned}
$$




$$
\begin{aligned}
& -k_{t r p} \sum_{i=1}^{\infty} \sum_{j=0}^{\infty} \sum_{k=0}^{\infty} \sum_{l=0}^{\infty} \sum_{m=0}^{\infty} \operatorname{lm} P_{i j k l m} \sum_{i_{1}=1}^{\infty} \sum_{j_{1}=0}^{\infty} \sum_{k_{1}=0}^{\infty} \sum_{l_{1}=0}^{\infty} \sum_{m_{1}=0}^{\infty} i_{1} P_{i_{1} j_{1} k_{1} l_{1} m_{1}} \\
& -k_{t r p} \sum_{i=1}^{\infty} \sum_{j=0}^{\infty} \sum_{k=0}^{\infty} \sum_{l=0}^{\infty} \sum_{m=0}^{\infty} i l P_{i j k l m} \sum_{i_{1}=1}^{\infty} \sum_{j_{1}=0}^{\infty} \sum_{k_{1}=0}^{\infty} \sum_{l_{1}=0}^{\infty} \sum_{m_{1}=0}^{\infty} m_{1} P_{i_{1} j_{1} k_{1} l_{1} m_{1}} \\
& +k_{\text {trp }} \sum_{i=1}^{\infty} \sum_{j=0}^{\infty} \sum_{k=0}^{\infty} \sum_{l=0}^{\infty} \sum_{m=0}^{\infty} \operatorname{lm} P_{i j k l m} \sum_{i_{1}=1}^{\infty} \sum_{j_{1}=0}^{\infty} \sum_{k_{1}=0}^{\infty} \sum_{l_{1}=0}^{\infty} \sum_{m_{1}=0}^{\infty} i_{1} P_{i_{1} j_{1} k_{1} l_{1} m_{1}} \\
& +k_{t r p} \sum_{i=1}^{\infty} \sum_{j=0}^{\infty} \sum_{k=0}^{\infty} \sum_{l=0}^{\infty} \sum_{m=0}^{\infty} i l P_{i j k l m} \sum_{i_{1}=1}^{\infty} \sum_{j_{1}=0}^{\infty} \sum_{k_{1}=0}^{\infty} \sum_{l_{1}=0}^{\infty} \sum_{m_{1}=0}^{\infty} m_{1} P_{i_{1} j_{1} k_{1} l_{1} m_{1}} \\
& -k_{i n t r} \sum_{i=1}^{\infty} \sum_{j=0}^{\infty} \sum_{k=0}^{\infty} \sum_{l=0}^{\infty} \sum_{m=0}^{\infty} k l P_{i j k l m} \\
& +k_{\text {intr }} \sum_{i=1}^{\infty} \sum_{j=0}^{\infty} \sum_{k=0}^{\infty} \sum_{l=0}^{\infty} \sum_{m=0}^{\infty} k l P_{i j k l m} \\
& -k_{r} \sum_{i=1}^{\infty} \sum_{j=0}^{\infty} \sum_{k=0}^{\infty} \sum_{l=0}^{\infty} \sum_{m=0}^{\infty} m l P_{i j k l m} \\
& +k_{r} \sum_{i=1}^{\infty} \sum_{j=0}^{\infty} \sum_{k=0}^{\infty} \sum_{l=0}^{\infty} \sum_{m=0}^{\infty} m l P_{i j k l m} \\
& -k_{t c} \sum_{i=1}^{\infty} \sum_{j=0}^{\infty} \sum_{k=0}^{\infty} \sum_{l=0}^{\infty} \sum_{m=0}^{\infty} k l P_{i j k l m} \sum_{i_{1}=1}^{\infty} \sum_{j_{1}=0}^{\infty} \sum_{k_{1}=0}^{\infty} \sum_{l_{1}=0}^{\infty} \sum_{m_{1}=0}^{\infty} k_{1} P_{i_{1} j_{1} k_{1} l_{1} m_{1}} \\
& +\frac{1}{2}(2) k_{t c} \sum_{i=1}^{\infty} \sum_{j=0}^{\infty} \sum_{k=0}^{\infty} \sum_{l=0}^{\infty} \sum_{m=0}^{\infty} k l P_{i j k l m} \sum_{i_{1}=1}^{\infty} \sum_{j_{1}=0}^{\infty} \sum_{k_{1}=0}^{\infty} \sum_{l_{1}=0}^{\infty} \sum_{m_{1}=0}^{\infty} k_{1} P_{i_{1} j_{1} k_{1} l_{1} m_{1}} \\
& -k_{t c} \sum_{i=1}^{\infty} \sum_{j=0}^{\infty} \sum_{k=0}^{\infty} \sum_{l=0}^{\infty} \sum_{m=0}^{\infty} \operatorname{lm} P_{i j k l m} \sum_{i_{1}=1}^{\infty} \sum_{j_{1}=0}^{\infty} \sum_{k_{1}=0}^{\infty} \sum_{l_{1}=0}^{\infty} \sum_{m_{1}=0}^{\infty} m_{1} P_{i_{1} j_{1} k_{1} l_{1} m_{1}} \\
& +\frac{1}{2}(2) k_{t c} \sum_{i=1}^{\infty} \sum_{j=0}^{\infty} \sum_{k=0}^{\infty} \sum_{l=0}^{\infty} \sum_{m=0}^{\infty} \operatorname{lm} P_{i j k l m} \sum_{i_{1}=1}^{\infty} \sum_{j_{1}=0}^{\infty} \sum_{k_{1}=0}^{\infty} \sum_{l_{1}=0}^{\infty} \sum_{m_{1}=0}^{\infty} m_{1} P_{i_{1} j_{1} k_{1} l_{1} m_{1}} \\
& -k_{t c} \sum_{i=1}^{\infty} \sum_{j=0}^{\infty} \sum_{k=0}^{\infty} \sum_{l=0}^{\infty} \sum_{m=0}^{\infty} k l P_{i j k l m} \sum_{i_{1}=1}^{\infty} \sum_{j_{1}=0}^{\infty} \sum_{k_{1}=0}^{\infty} \sum_{l_{1}=0}^{\infty} \sum_{m_{1}=0}^{\infty} m_{1} P_{i_{1} j_{1} k_{1} l_{1} m_{1}} \\
& -k_{t c} \sum_{i=1}^{\infty} \sum_{j=0}^{\infty} \sum_{k=0}^{\infty} \sum_{l=0}^{\infty} \sum_{m=0}^{\infty} \operatorname{lm} P_{i j k l m} \sum_{i_{1}=1}^{\infty} \sum_{j_{1}=0}^{\infty} \sum_{k_{1}=0}^{\infty} \sum_{l_{1}=0}^{\infty} \sum_{m_{1}=0}^{\infty} k_{1} P_{i_{1} j_{1} k_{1}} l_{1} m_{1} \\
& +\frac{1}{2}(2) k_{t c} \sum_{i=1}^{\infty} \sum_{j=0}^{\infty} \sum_{k=0}^{\infty} \sum_{l=0}^{\infty} \sum_{m=0}^{\infty} k l P_{i j k l m} \sum_{i_{1}=1}^{\infty} \sum_{j_{1}=0}^{\infty} \sum_{k_{1}=0}^{\infty} \sum_{l_{1}=0}^{\infty} \sum_{m_{1}=0}^{\infty} m_{1} P_{i_{1} j_{1} k_{1} l_{1} m_{1}} \\
& +\frac{1}{2}(2) k_{t c} \sum_{i=1}^{\infty} \sum_{j=0}^{\infty} \sum_{k=0}^{\infty} \sum_{l=0}^{\infty} \sum_{m=0}^{\infty} \operatorname{lm} P_{i j k l m} \sum_{i_{1}=1}^{\infty} \sum_{j_{1}=0}^{\infty} \sum_{k_{1}=0}^{\infty} \sum_{l_{1}=0}^{\infty} \sum_{m_{1}=0}^{\infty} k_{1} P_{i_{1} j_{1} k_{1} l_{1} m_{1}}
\end{aligned}
$$

using Equations (42) and (43).

$$
\Rightarrow \frac{d \mu_{00010}}{d t}=k_{t r t} C_{T} \sum_{i=1}^{\infty} \sum_{j=0}^{\infty} \sum_{k=0}^{\infty} \sum_{l=0}^{\infty} \sum_{m=0}^{\infty} k P_{i j k l m}
$$


$\Rightarrow \frac{d \mu_{000010}}{d t}=k_{t r t} C_{T} \mu_{00100}$ 
3.2.5 First moment w.r.t. the number of unrelaxed (just uncapped) radicals

$$
\begin{aligned}
& \frac{d \mu_{00001}}{d t}=\sum_{i=1}^{\infty} \sum_{j=0}^{\infty} \sum_{k=0}^{\infty} \sum_{l=0}^{\infty} \sum_{m=0}^{\infty} m \frac{d P_{i j k l m}}{d t} \\
& =-k_{p} C_{M} \sum_{i=1}^{\infty} \sum_{j=0}^{\infty} \sum_{k=0}^{\infty} \sum_{l=0}^{\infty} \sum_{m=0}^{\infty} k m P_{i j k l m} \\
& +k_{p} C_{M} \sum_{i=2}^{\infty} \sum_{j=0}^{\infty} \sum_{k=0}^{\infty} \sum_{l=0}^{\infty} \sum_{m=0}^{\infty} k m P_{i-1 j k l m} \\
& -k_{p} C_{M} \sum_{i=1}^{\infty} \sum_{j=0}^{\infty} \sum_{k=0}^{\infty} \sum_{l=0}^{\infty} \sum_{m=0}^{\infty} m^{2} P_{i j k l m} \\
& +k_{p} C_{M} \sum_{i=2}^{\infty} \sum_{j=0}^{\infty} \sum_{k=0}^{\infty} \sum_{l=0}^{\infty} \sum_{m=0}^{\infty} m^{2} P_{i-1 j k l m} \\
& -k_{t r t} C_{T} \sum_{i=1}^{\infty} \sum_{j=0}^{\infty} \sum_{k=0}^{\infty} \sum_{l=0}^{\infty} \sum_{m=0}^{\infty} k m P_{i j k l m} \\
& +k_{t r t} C_{T} \sum_{i=1}^{\infty} \sum_{j=0}^{\infty} \sum_{k=0}^{\infty} \sum_{l=1}^{\infty} \sum_{m=0}^{\infty}(k+1) m P_{i j k+1 l-1 m} \\
& -k_{t r t} \sum_{i=1}^{\infty} \sum_{j=0}^{\infty} \sum_{k=0}^{\infty} \sum_{l=0}^{\infty} \sum_{m=0}^{\infty} k m P_{i j k l m} \sum_{i_{1}=1}^{\infty} \sum_{j_{1}=0}^{\infty} \sum_{k_{1}=0}^{\infty} \sum_{l_{1}=0}^{\infty} \sum_{m_{1}=0}^{\infty} l_{1} P_{i_{1} j_{1} k_{1} l_{1} m_{1}} \\
& -k_{\text {trt }} \sum_{i=1}^{\infty} \sum_{j=0}^{\infty} \sum_{k=0}^{\infty} \sum_{l=0}^{\infty} \sum_{m=0}^{\infty} \operatorname{lm} P_{i j k l m} \sum_{i_{1}=1}^{\infty} \sum_{j_{1}=0}^{\infty} \sum_{k_{1}=0}^{\infty} \sum_{l_{1}=0}^{\infty} \sum_{m_{1}=0}^{\infty} k_{1} P_{i_{1} j_{1} k_{1} l_{1} m_{1}} \\
& +k_{t r t} \sum_{i=1}^{\infty} \sum_{j=0}^{\infty} \sum_{k=0}^{\infty} \sum_{l=1}^{\infty} \sum_{m=0}^{\infty}(k+1) m P_{i j k+1 l-1 m} \sum_{i_{1}=1}^{\infty} \sum_{j_{1}=0}^{\infty} \sum_{k_{1}=0}^{\infty} \sum_{l_{1}=0}^{\infty} \sum_{m_{1}=0}^{\infty} l_{1} P_{i_{1} j_{1} k_{1} l_{1} m_{1}} \\
& +k_{\text {trt }} \sum_{i=1}^{\infty} \sum_{j=0}^{\infty} \sum_{k=0}^{\infty} \sum_{l=0}^{\infty} \sum_{m=1}^{\infty}(l+1) m P_{i j k l+1 m-1} \sum_{i_{1}=1}^{\infty} \sum_{j_{1}=0}^{\infty} \sum_{k_{1}=0}^{\infty} \sum_{l_{1}=0}^{\infty} \sum_{m_{1}=0}^{\infty} k_{1} P_{i_{1} j_{1} k_{1} l_{1} m_{1}} \\
& -k_{\operatorname{trp}} \sum_{i=1}^{\infty} \sum_{j=0}^{\infty} \sum_{k=0}^{\infty} \sum_{l=0}^{\infty} \sum_{m=0}^{\infty} k m P_{i j k l m} \sum_{i_{1}=1}^{\infty} \sum_{j_{1}=0}^{\infty} \sum_{k_{1}=0}^{\infty} \sum_{l_{1}=0}^{\infty} \sum_{m_{1}=0}^{\infty} i_{1} P_{i_{1} j_{1} k_{1} l_{1} m_{1}} \\
& -k_{\operatorname{trp}} \sum_{i=1}^{\infty} \sum_{j=0}^{\infty} \sum_{k=0}^{\infty} \sum_{l=0}^{\infty} \sum_{m=0}^{\infty} i m P_{i j k l m} \sum_{i_{1}=1}^{\infty} \sum_{j_{1}=0}^{\infty} \sum_{k_{1}=0}^{\infty} \sum_{l_{1}=0}^{\infty} \sum_{m_{1}=0}^{\infty} k_{1} P_{i_{1} j_{1} k_{1} l_{1} m_{1}} \\
& +k_{\operatorname{trp}} \sum_{i=1}^{\infty} \sum_{j=0}^{\infty} \sum_{k=0}^{\infty} \sum_{l=0}^{\infty} \sum_{m=0}^{\infty}(k+1) m P_{i j k+1 l m} \sum_{i_{1}=1}^{\infty} \sum_{j_{1}=0}^{\infty} \sum_{k_{1}=0}^{\infty} \sum_{l_{1}=0}^{\infty} \sum_{m_{1}=0}^{\infty} i_{1} P_{i_{1} j_{1} k_{1} l_{1} m_{1}} \\
& +k_{\operatorname{trp}} \sum_{i=1}^{\infty} \sum_{j=1}^{\infty} \sum_{k=1}^{\infty} \sum_{l=0}^{\infty} \sum_{m=0}^{\infty} i m P_{i j-1 k-1 l m} \sum_{i_{1}=1}^{\infty} \sum_{j_{1}=0}^{\infty} \sum_{k_{1}=0}^{\infty} \sum_{l_{1}=0}^{\infty} \sum_{m_{1}=0}^{\infty} k_{1} P_{i_{1} j_{1} k_{1} l_{1} m_{1}}
\end{aligned}
$$




$$
\begin{aligned}
& -k_{\operatorname{trp}} \sum_{i=1}^{\infty} \sum_{j=0}^{\infty} \sum_{k=0}^{\infty} \sum_{l=0}^{\infty} \sum_{m=0}^{\infty} m^{2} P_{i j k l m} \sum_{i_{1}=1}^{\infty} \sum_{j_{1}=0}^{\infty} \sum_{k_{1}=0}^{\infty} \sum_{l_{1}=0}^{\infty} \sum_{m_{1}=0}^{\infty} i_{1} P_{i_{1} j_{1} k_{1} l_{1} m_{1}} \\
& -k_{t r p} \sum_{i=1}^{\infty} \sum_{j=0}^{\infty} \sum_{k=0}^{\infty} \sum_{l=0}^{\infty} \sum_{m=0}^{\infty} i m P_{i j k l m} \sum_{i_{1}=1}^{\infty} \sum_{j_{1}=0}^{\infty} \sum_{k_{1}=0}^{\infty} \sum_{l_{1}=0}^{\infty} \sum_{m_{1}=0}^{\infty} m_{1} P_{i_{1} j_{1} k_{1} l_{1} m_{1}} \\
& +k_{t r p} \sum_{i=1}^{\infty} \sum_{j=0}^{\infty} \sum_{k=0}^{\infty} \sum_{l=0}^{\infty} \sum_{m=0}^{\infty} m(m+1) P_{i j k l m+1} \sum_{i_{1}=1}^{\infty} \sum_{j_{1}=0}^{\infty} \sum_{k_{1}=0}^{\infty} \sum_{l_{1}=0}^{\infty} \sum_{m_{1}=0}^{\infty} i_{1} P_{i_{1} j_{1} k_{1} l_{1} m_{1}} \\
& +k_{t r p} \sum_{i=1}^{\infty} \sum_{j=1}^{\infty} \sum_{k=0}^{\infty} \sum_{l=0}^{\infty} \sum_{m=1}^{\infty} i m P_{i j-1 k l m-1} \sum_{i_{1}=1}^{\infty} \sum_{j_{1}=0}^{\infty} \sum_{k_{1}=0}^{\infty} \sum_{l_{1}=0}^{\infty} \sum_{m_{1}=0}^{\infty} m_{1} P_{i_{1} j_{1} k_{1} l_{1} m_{1}} \\
& -k_{i n t r} \sum_{i=1}^{\infty} \sum_{j=0}^{\infty} \sum_{k=0}^{\infty} \sum_{l=0}^{\infty} \sum_{m=0}^{\infty} k m P_{i j k l m} \\
& +k_{i n t r} \sum_{i=1}^{\infty} \sum_{j=1}^{\infty} \sum_{k=0}^{\infty} \sum_{l=0}^{\infty} \sum_{m=0}^{\infty} k m P_{i j-1 k l m} \\
& -k_{r} \sum_{i=1}^{\infty} \sum_{j=0}^{\infty} \sum_{k=0}^{\infty} \sum_{l=0}^{\infty} \sum_{m=0}^{\infty} m^{2} P_{i j k l m} \\
& +k_{r} \sum_{i=1}^{\infty} \sum_{j=0}^{\infty} \sum_{k=1}^{\infty} \sum_{l=0}^{\infty} \sum_{m=0}^{\infty} m(m+1) P_{i j k-1 l m+1} \\
& -k_{t c} \sum_{i=1}^{\infty} \sum_{j=0}^{\infty} \sum_{k=0}^{\infty} \sum_{l=0}^{\infty} \sum_{m=0}^{\infty} k m P_{i j k l m} \sum_{i_{1}=1}^{\infty} \sum_{j_{1}=0}^{\infty} \sum_{k_{1}=0}^{\infty} \sum_{l_{1}=0}^{\infty} \sum_{m_{1}=0}^{\infty} k_{1} P_{i_{1} j_{1} k_{1} l_{1} m_{1}} \\
& +\frac{1}{2} k_{t c} \sum_{i=1}^{\infty} \sum_{j=0}^{\infty} \sum_{k=0}^{\infty} \sum_{l=0}^{\infty} \sum_{m=0}^{\infty} \sum_{i_{1}=1}^{i} \sum_{j_{1}=0}^{j} \sum_{k_{1}=0}^{k+2} \sum_{l_{1}=0}^{l} \sum_{m_{1}=0}^{m} m\left[k_{1} P_{i_{1} j_{1} k_{1} l_{1} m_{1}}\left(k+2-k_{1}\right) P_{i-i_{1}, j-j_{1}, k+2-k_{1}, l-l_{1} m-m_{1}}\right] \\
& -k_{t c} \sum_{i=1}^{\infty} \sum_{j=0}^{\infty} \sum_{k=0}^{\infty} \sum_{l=0}^{\infty} \sum_{m=0}^{\infty} m^{2} P_{i j k l m} \sum_{i_{1}=1}^{\infty} \sum_{j_{1}=0}^{\infty} \sum_{k_{1}=0}^{\infty} \sum_{l_{1}=0}^{\infty} \sum_{m_{1}=0}^{\infty} m_{1} P_{i_{1} j_{1} k_{1} l_{1} m_{1}} \\
& +\frac{1}{2} k_{t c} \sum_{i=1}^{\infty} \sum_{j=0}^{\infty} \sum_{k=0}^{\infty} \sum_{l=0}^{\infty} \sum_{m=0}^{\infty} \sum_{i_{1}=1}^{i} \sum_{j_{1}=0}^{j} \sum_{k_{1}=0}^{k} \sum_{l_{1}=0}^{l} \sum_{m_{1}=0}^{m+2}\left[m m_{1} P_{i_{1} j_{1} k_{1} l_{1} m_{1}}\left(m+2-m_{1}\right) P_{i-i_{1}, j-j_{1}, k-k_{1}, l-l_{1} m+2-m_{1}}\right] \\
& -k_{t c} \sum_{i=1}^{\infty} \sum_{j=0}^{\infty} \sum_{k=0}^{\infty} \sum_{l=0}^{\infty} \sum_{m=0}^{\infty} k m P_{i j k l m} \sum_{i_{1}=1}^{\infty} \sum_{j_{1}=0}^{\infty} \sum_{k_{1}=0}^{\infty} \sum_{l_{1}=0}^{\infty} \sum_{m_{1}=0}^{\infty} m_{1} P_{i_{1} j_{1} k_{1} l_{1} m_{1}} \\
& -k_{t c} \sum_{i=1}^{\infty} \sum_{j=0}^{\infty} \sum_{k=0}^{\infty} \sum_{l=0}^{\infty} \sum_{m=0}^{\infty} m^{2} P_{i j k l m} \sum_{i_{1}=1}^{\infty} \sum_{j_{1}=0}^{\infty} \sum_{k_{1}=0}^{\infty} \sum_{l_{1}=0}^{\infty} \sum_{m_{1}=0}^{\infty} k_{1} P_{i_{1} j_{1} k_{1} l_{1} m_{1}} \\
& +\frac{1}{2} k_{t c} \sum_{i=1}^{\infty} \sum_{j=0}^{\infty} \sum_{k=0}^{\infty} \sum_{l=0}^{\infty} \sum_{m=0}^{\infty} \sum_{i_{1}=1}^{i} \sum_{j_{1}=0}^{j} \sum_{k_{1}=0}^{k+1} \sum_{l_{1}=0}^{l} \sum_{m_{1}=0}^{m+1}\left[m k_{1} P_{i_{1} j_{1} k_{1} l_{1} m_{1}}\left(m+1-m_{1}\right) P_{\left.i-i_{1}, j-j_{1}, k+1-k_{1}, l-l_{1} m+1-m_{1}\right]}\right. \\
& +\frac{1}{2} k_{t c} \sum_{i=1}^{\infty} \sum_{j=0}^{\infty} \sum_{k=0}^{\infty} \sum_{l=0}^{\infty} \sum_{m=0}^{\infty} \sum_{i_{1}=1}^{i} \sum_{j_{1}=0}^{j} \sum_{k_{1}=0}^{k+1} \sum_{l_{1}=0}^{l} \sum_{m_{1}=0}^{m+1}\left[m m_{1} P_{i_{1} j_{1} k_{1} l_{1} m_{1}}\left(k+1-k_{1}\right) P_{\left.i-i_{1}, j-j_{1}, k+1-k_{1}, l-l_{1} m+1-m_{1}\right]}\right.
\end{aligned}
$$




$$
\begin{aligned}
& \Rightarrow \frac{d \mu_{00001}}{d t}=-k_{p} C_{M} \sum_{i=1}^{\infty} \sum_{j=0}^{\infty} \sum_{k=0}^{\infty} \sum_{l=0}^{\infty} \sum_{m=0}^{\infty} k m P_{i j k l m} \\
& +k_{p} C_{M} \sum_{i=1}^{\infty} \sum_{j=0}^{\infty} \sum_{k=0}^{\infty} \sum_{l=0}^{\infty} \sum_{m=0}^{\infty} k m P_{i j k l m} \\
& -k_{p} C_{M} \sum_{i=1}^{\infty} \sum_{j=0}^{\infty} \sum_{k=0}^{\infty} \sum_{l=0}^{\infty} \sum_{m=0}^{\infty} m^{2} P_{i j k l m} \\
& +k_{p} C_{M} \sum_{i=1}^{\infty} \sum_{j=0}^{\infty} \sum_{k=0}^{\infty} \sum_{l=0}^{\infty} \sum_{m=0}^{\infty} m^{2} P_{i j k l m} \\
& -k_{t r t} C_{T} \sum_{i=1}^{\infty} \sum_{j=0}^{\infty} \sum_{k=0}^{\infty} \sum_{l=0}^{\infty} \sum_{m=0}^{\infty} k m P_{i j k l m} \\
& +k_{t r t} C_{T} \sum_{i=1}^{\infty} \sum_{j=0}^{\infty} \sum_{k=0}^{\infty} \sum_{l=0}^{\infty} \sum_{m=0}^{\infty} k m P_{i j k l m} \\
& -k_{t r t} \sum_{i=1}^{\infty} \sum_{j=0}^{\infty} \sum_{k=0}^{\infty} \sum_{l=0}^{\infty} \sum_{m=0}^{\infty} k m P_{i j k l m} \sum_{i_{1}=1}^{\infty} \sum_{j_{1}=0}^{\infty} \sum_{k_{1}=0}^{\infty} \sum_{l_{1}=0}^{\infty} \sum_{m_{1}=0}^{\infty} l_{1} P_{i_{1} j_{1} k_{1} l_{1} m_{1}} \\
& -k_{t r t} \sum_{i=1}^{\infty} \sum_{j=0}^{\infty} \sum_{k=0}^{\infty} \sum_{l=0}^{\infty} \sum_{m=0}^{\infty} l m P_{i j k l m} \sum_{i_{1}=1}^{\infty} \sum_{j_{1}=0}^{\infty} \sum_{k_{1}=0}^{\infty} \sum_{l_{1}=0}^{\infty} \sum_{m_{1}=0}^{\infty} k_{1} P_{i_{1} j_{1} k_{1} l_{1} m_{1}} \\
& +k_{t r t} \sum_{i=1}^{\infty} \sum_{j=0}^{\infty} \sum_{k=0}^{\infty} \sum_{l=0}^{\infty} \sum_{m=0}^{\infty} k m P_{i j k l m} \sum_{i_{1}=1}^{\infty} \sum_{j_{1}=0}^{\infty} \sum_{k_{1}=0}^{\infty} \sum_{l_{1}=0}^{\infty} \sum_{m_{1}=0}^{\infty} l_{1} P_{i_{1} j_{1} k_{1} l_{1} m_{1}} \\
& +k_{t r t} \sum_{i=1}^{\infty} \sum_{j=0}^{\infty} \sum_{k=0}^{\infty} \sum_{l=0}^{\infty} \sum_{m=0}^{\infty} l(m+1) P_{i j k l m} \sum_{i_{1}=1}^{\infty} \sum_{j_{1}=0}^{\infty} \sum_{k_{1}=0}^{\infty} \sum_{l_{1}=0}^{\infty} \sum_{m_{1}=0}^{\infty} k_{1} P_{i_{1} j_{1} k_{1} l_{1} m_{1}} \\
& -k_{\text {trp }} \sum_{i=1}^{\infty} \sum_{j=0}^{\infty} \sum_{k=0}^{\infty} \sum_{l=0}^{\infty} \sum_{m=0}^{\infty} k m P_{i j k l m} \sum_{i_{1}=1}^{\infty} \sum_{j_{1}=0}^{\infty} \sum_{k_{1}=0}^{\infty} \sum_{l_{1}=0}^{\infty} \sum_{m_{1}=0}^{\infty} i_{1} P_{i_{1} j_{1} k_{1} l_{1} m_{1}} \\
& -k_{\text {trp }} \sum_{i=1}^{\infty} \sum_{j=0}^{\infty} \sum_{k=0}^{\infty} \sum_{l=0}^{\infty} \sum_{m=0}^{\infty} i m P_{i j k l m} \sum_{i_{1}=1}^{\infty} \sum_{j_{1}=0}^{\infty} \sum_{k_{1}=0}^{\infty} \sum_{l_{1}=0}^{\infty} \sum_{m_{1}=0}^{\infty} k_{1} P_{i_{1} j_{1} k_{1} l_{1} m_{1}} \\
& +k_{t r p} \sum_{i=1}^{\infty} \sum_{j=0}^{\infty} \sum_{k=0}^{\infty} \sum_{l=0}^{\infty} \sum_{m=0}^{\infty} k m P_{i j k l m} \sum_{i_{1}=1}^{\infty} \sum_{j_{1}=0}^{\infty} \sum_{k_{1}=0}^{\infty} \sum_{l_{1}=0}^{\infty} \sum_{m_{1}=0}^{\infty} i_{1} P_{i_{1} j_{1} k_{1} l_{1} m_{1}} \\
& +k_{\text {trp }} \sum_{i=1}^{\infty} \sum_{j=0}^{\infty} \sum_{k=0}^{\infty} \sum_{l=0}^{\infty} \sum_{m=0}^{\infty} i m P_{i j k l m} \sum_{i_{1}=1}^{\infty} \sum_{j_{1}=0}^{\infty} \sum_{k_{1}=0}^{\infty} \sum_{l_{1}=0}^{\infty} \sum_{m_{1}=0}^{\infty} k_{1} P_{i_{1} j_{1} k_{1} l_{1} m_{1}}
\end{aligned}
$$




$$
\begin{aligned}
& -k_{t r p} \sum_{i=1}^{\infty} \sum_{j=0}^{\infty} \sum_{k=0}^{\infty} \sum_{l=0}^{\infty} \sum_{m=0}^{\infty} m^{2} P_{i j k l m} \sum_{i_{1}=1}^{\infty} \sum_{j_{1}=0}^{\infty} \sum_{k_{1}=0}^{\infty} \sum_{l_{1}=0}^{\infty} \sum_{m_{1}=0}^{\infty} i_{1} P_{i_{1} j_{1} k_{1} l_{1} m_{1}} \\
& -k_{t r p} \sum_{i=1}^{\infty} \sum_{j=0}^{\infty} \sum_{k=0}^{\infty} \sum_{l=0}^{\infty} \sum_{m=0}^{\infty} i m P_{i j k l m} \sum_{i_{1}=1}^{\infty} \sum_{j_{1}=0}^{\infty} \sum_{k_{1}=0}^{\infty} \sum_{l_{1}=0}^{\infty} \sum_{m_{1}=0}^{\infty} m_{1} P_{i_{1} j_{1} k_{1} l_{1} m_{1}} \\
& +k_{t r p} \sum_{i=1}^{\infty} \sum_{j=0}^{\infty} \sum_{k=0}^{\infty} \sum_{l=0}^{\infty} \sum_{m=0}^{\infty}(m-1) m P_{i j k l m} \sum_{i_{1}=1}^{\infty} \sum_{j_{1}=0}^{\infty} \sum_{k_{1}=0}^{\infty} \sum_{l_{1}=0}^{\infty} \sum_{m_{1}=0}^{\infty} i_{1} P_{i_{1} j_{1} k_{1} l_{1} m_{1}} \\
& +k_{t r p} \sum_{i=1}^{\infty} \sum_{j=0}^{\infty} \sum_{k=0}^{\infty} \sum_{l=0}^{\infty} \sum_{m=0}^{\infty} i(m+1) P_{i j k l m} \sum_{i_{1}=1}^{\infty} \sum_{j_{1}=0}^{\infty} \sum_{k_{1}=0}^{\infty} \sum_{l_{1}=0}^{\infty} \sum_{m_{1}=0}^{\infty} m_{1} P_{i_{1} j_{1} k_{1} l_{1} m_{1}} \\
& -k_{i n t r} \sum_{i=1}^{\infty} \sum_{j=0}^{\infty} \sum_{k=0}^{\infty} \sum_{l=0}^{\infty} \sum_{m=0}^{\infty} k m P_{i j k l m} \\
& +k_{i n t r} \sum_{i=1}^{\infty} \sum_{j=0}^{\infty} \sum_{k=0}^{\infty} \sum_{l=0}^{\infty} \sum_{m=0}^{\infty} k m P_{i j k l m} \\
& -k_{r} \sum_{i=1}^{\infty} \sum_{j=0}^{\infty} \sum_{k=0}^{\infty} \sum_{l=0}^{\infty} \sum_{m=0}^{\infty} m^{2} P_{i j k l m} \\
& +k_{r} \sum_{i=1}^{\infty} \sum_{j=0}^{\infty} \sum_{k=0}^{\infty} \sum_{l=0}^{\infty} \sum_{m=0}^{\infty}(m-1) m P_{i j k l m} \\
& -k_{t c} \sum_{i=1}^{\infty} \sum_{j=0}^{\infty} \sum_{k=0}^{\infty} \sum_{l=0}^{\infty} \sum_{m=0}^{\infty} k m P_{i j k l m} \sum_{i_{1}=1}^{\infty} \sum_{j_{1}=0}^{\infty} \sum_{k_{1}=0}^{\infty} \sum_{l_{1}=0}^{\infty} \sum_{m_{1}=0}^{\infty} k_{1} P_{i_{1} j_{1} k_{1} l_{1} m_{1}} \\
& +\frac{1}{2}(2) k_{t c} \sum_{i=1}^{\infty} \sum_{j=0}^{\infty} \sum_{k=0}^{\infty} \sum_{l=0}^{\infty} \sum_{m=0}^{\infty} k m P_{i j k l m} \sum_{i_{1}=1}^{\infty} \sum_{j_{1}=0}^{\infty} \sum_{k_{1}=0}^{\infty} \sum_{l_{1}=0}^{\infty} \sum_{m_{1}=0}^{\infty} k_{1} P_{i_{1} j_{1} k_{1} l_{1} m_{1}} \\
& -k_{t c} \sum_{i=1}^{\infty} \sum_{j=0}^{\infty} \sum_{k=0}^{\infty} \sum_{l=0}^{\infty} \sum_{m=0}^{\infty} m^{2} P_{i j k l m} \sum_{i_{1}=1}^{\infty} \sum_{j_{1}=0}^{\infty} \sum_{k_{1}=0}^{\infty} \sum_{l_{1}=0}^{\infty} \sum_{m_{1}=0}^{\infty} m_{1} P_{i_{1} j_{1} k_{1} l_{1} m_{1}} \\
& +\frac{1}{2}(2) k_{t c} \sum_{i=1}^{\infty} \sum_{j=0}^{\infty} \sum_{k=0}^{\infty} \sum_{l=0}^{\infty} \sum_{m=0}^{\infty} m^{2} P_{i j k l m} \sum_{i_{1}=1}^{\infty} \sum_{j_{1}=0}^{\infty} \sum_{k_{1}=0}^{\infty} \sum_{l_{1}=0}^{\infty} \sum_{m_{1}=0}^{\infty} m_{1} P_{i_{1} j_{1} k_{1} l_{1} m_{1}} \\
& -\frac{1}{2}(2) k_{t c} \sum_{i=1}^{\infty} \sum_{j=0}^{\infty} \sum_{k=0}^{\infty} \sum_{l=0}^{\infty} \sum_{m=0}^{\infty} m P_{i j k l m} \sum_{i_{1}=1}^{\infty} \sum_{j_{1}=0}^{\infty} \sum_{k_{1}=0}^{\infty} \sum_{l_{1}=0}^{\infty} \sum_{m_{1}=0}^{\infty} m_{1} P_{i_{1} j_{1} k_{1} l_{1} m_{1}} \\
& -k_{t c} \sum_{i=1}^{\infty} \sum_{j=0}^{\infty} \sum_{k=0}^{\infty} \sum_{l=0}^{\infty} \sum_{m=0}^{\infty} k m P_{i j k l m} \sum_{i_{1}=1}^{\infty} \sum_{j_{1}=0}^{\infty} \sum_{k_{1}=0}^{\infty} \sum_{l_{1}=0}^{\infty} \sum_{m_{1}=0}^{\infty} m_{1} P_{i_{1} j_{1} k_{1} l_{1} m_{1}} \\
& -k_{t c} \sum_{i=1}^{\infty} \sum_{j=0}^{\infty} \sum_{k=0}^{\infty} \sum_{l=0}^{\infty} \sum_{m=0}^{\infty} m^{2} P_{i j k l m} \sum_{i_{1}=1}^{\infty} \sum_{j_{1}=0}^{\infty} \sum_{k_{1}=0}^{\infty} \sum_{l_{1}=0}^{\infty} \sum_{m_{1}=0}^{\infty} k_{1} P_{i_{1} j_{1} k_{1} l_{1} m_{1}} \\
& +\frac{1}{2}(2) k_{t c} \sum_{i=1}^{\infty} \sum_{j=0}^{\infty} \sum_{k=0}^{\infty} \sum_{l=0}^{\infty} \sum_{m=0}^{\infty} k m P_{i j k l m} \sum_{i_{1}=1}^{\infty} \sum_{j_{1}=0}^{\infty} \sum_{k_{1}=0}^{\infty} \sum_{l_{1}=0}^{\infty} \sum_{m_{1}=0}^{\infty} m_{1} P_{i_{1} j_{1} k_{1} l_{1} m_{1}} \\
& +\frac{1}{2}(2) k_{t c} \sum_{i=1}^{\infty} \sum_{j=0}^{\infty} \sum_{k=0}^{\infty} \sum_{l=0}^{\infty} \sum_{m=0}^{\infty} m^{2} P_{i j k l m} \sum_{i_{1}=1}^{\infty} \sum_{j_{1}=0}^{\infty} \sum_{k_{1}=0}^{\infty} \sum_{l_{1}=0}^{\infty} \sum_{m_{1}=0}^{\infty} k_{1} P_{i_{1} j_{1} k_{1} l_{1} m_{1}} \\
& -\frac{1}{2}(2) k_{t c} \sum_{i=1}^{\infty} \sum_{j=0}^{\infty} \sum_{k=0}^{\infty} \sum_{l=0}^{\infty} \sum_{m=0}^{\infty} m P_{i j k l m} \sum_{i_{1}=1}^{\infty} \sum_{j_{1}=0}^{\infty} \sum_{k_{1}=0}^{\infty} \sum_{l_{1}=0}^{\infty} \sum_{m_{1}=0}^{\infty} k_{1} P_{i_{1} j_{1} k_{1} l_{1} m_{1}}
\end{aligned}
$$

using Equations (42), (52), (53) and (54). 


$$
\begin{aligned}
& \Rightarrow \frac{d \mu_{00001}}{d t}=k_{t r t} \sum_{i=1}^{\infty} \sum_{j=0}^{\infty} \sum_{k=0}^{\infty} \sum_{l=0}^{\infty} \sum_{m=0}^{\infty} l P_{i j k l m} \sum_{i_{1}=1}^{\infty} \sum_{j_{1}=0}^{\infty} \sum_{k_{1}=0}^{\infty} \sum_{l_{1}=0}^{\infty} \sum_{m_{1}=0}^{\infty} k_{1} P_{i_{1} j_{1} k_{1} l_{1} m_{1}} \\
& \quad-k_{\text {trp }} \sum_{i=1}^{\infty} \sum_{j=0}^{\infty} \sum_{k=0}^{\infty} \sum_{l=0}^{\infty} \sum_{m=0}^{\infty} m P_{i j k l m} \sum_{i_{1}=1}^{\infty} \sum_{j_{1}=0}^{\infty} \sum_{k_{1}=0}^{\infty} \sum_{l_{1}=0}^{\infty} \sum_{m_{1}=0}^{\infty} i_{1} P_{i_{1} j_{1} k_{1} l_{1} m_{1}} \\
& \quad+k_{t r p} \sum_{i=1}^{\infty} \sum_{j=0}^{\infty} \sum_{k=0}^{\infty} \sum_{l=0}^{\infty} \sum_{m=0}^{\infty} i P_{i j k l m} \sum_{i_{1}=1}^{\infty} \sum_{j_{1}=0}^{\infty} \sum_{k_{1}=0}^{\infty} \sum_{l_{1}=0}^{\infty} \sum_{m_{1}=0}^{\infty} m_{1} P_{i_{1} j_{1} k_{1} l_{1} m_{1}} \\
& \quad-k_{r} \sum_{i=1}^{\infty} \sum_{j=0}^{\infty} \sum_{k=0}^{\infty} \sum_{l=0}^{\infty} \sum_{m=0}^{\infty} m P_{i j k l m} \\
& -k_{t c} \sum_{i=1}^{\infty} \sum_{j=0}^{\infty} \sum_{k=0}^{\infty} \sum_{l=0}^{\infty} \sum_{m=0}^{\infty} m P_{i j k l m} \sum_{i_{1}=1}^{\infty} \sum_{j_{1}=0}^{\infty} \sum_{k_{1}=0}^{\infty} \sum_{l_{1}=0}^{\infty} \sum_{m_{1}=0}^{\infty} m_{1} P_{i_{1} j_{1} k_{1} l_{1} m_{1}} \\
& -k_{t c} \sum_{i=1}^{\infty} \sum_{j=0}^{\infty} \sum_{k=0}^{\infty} \sum_{l=0}^{\infty} \sum_{m=0}^{\infty} k P_{i j k l m} \sum_{i_{1}=1}^{\infty} \sum_{j_{1}=0}^{\infty} \sum_{k_{1}=0}^{\infty} \sum_{l_{1}=0}^{\infty} \sum_{m_{1}=0}^{\infty} m_{1} P_{i_{1} j_{1} k_{1} l_{1} m_{1}}
\end{aligned}
$$




\subsection{Second moments}

\subsubsection{Second moment w.r.t. the number of repeat units}

$$
\begin{aligned}
& \frac{d \mu_{20000}}{d t}=\sum_{i=1}^{\infty} \sum_{j=0}^{\infty} \sum_{k=0}^{\infty} \sum_{l=0}^{\infty} \sum_{m=0}^{\infty} i^{2} \frac{d P_{i j k l m}}{d t} \\
& =k_{i} C_{M} C_{R} \\
& -k_{p} C_{M} \sum_{i=1}^{\infty} \sum_{j=0}^{\infty} \sum_{k=0}^{\infty} \sum_{l=0}^{\infty} \sum_{m=0}^{\infty} i^{2} k P_{i j k l m} \\
& +k_{p} C_{M} \sum_{i=2}^{\infty} \sum_{j=0}^{\infty} \sum_{k=0}^{\infty} \sum_{l=0}^{\infty} \sum_{m=0}^{\infty} i^{2} k P_{i-1 j k l m} \\
& -k_{p} C_{M} \sum_{i=1}^{\infty} \sum_{j=0}^{\infty} \sum_{k=0}^{\infty} \sum_{l=0}^{\infty} \sum_{m=0}^{\infty} i^{2} m P_{i j k l m} \\
& +k_{p} C_{M} \sum_{i=2}^{\infty} \sum_{j=0}^{\infty} \sum_{k=0}^{\infty} \sum_{l=0}^{\infty} \sum_{m=0}^{\infty} i^{2} m P_{i-1 j k l m} \\
& -k_{t r t} C_{T} \sum_{i=1}^{\infty} \sum_{j=0}^{\infty} \sum_{k=0}^{\infty} \sum_{l=0}^{\infty} \sum_{m=0}^{\infty} i^{2} k P_{i j k l m} \\
& +k_{t r t} C_{T} \sum_{i=1}^{\infty} \sum_{j=0}^{\infty} \sum_{k=0}^{\infty} \sum_{l=1}^{\infty} \sum_{m=0}^{\infty} i^{2}(k+1) P_{i j k+1 l-1 m} \\
& -k_{\text {trt }} \sum_{i=1}^{\infty} \sum_{j=0}^{\infty} \sum_{k=0}^{\infty} \sum_{l=0}^{\infty} \sum_{m=0}^{\infty} i^{2} k P_{i j k l m} \sum_{i_{1}=1}^{\infty} \sum_{j_{1}=0}^{\infty} \sum_{k_{1}=0}^{\infty} \sum_{l_{1}=0}^{\infty} \sum_{m_{1}=0}^{\infty} l_{1} P_{i_{1} j_{1} k_{1} l_{1} m_{1}} \\
& -k_{\text {trt }} \sum_{i=1}^{\infty} \sum_{j=0}^{\infty} \sum_{k=0}^{\infty} \sum_{l=0}^{\infty} \sum_{m=0}^{\infty} i^{2} l P_{i j k l m} \sum_{i_{1}=1}^{\infty} \sum_{j_{1}=0}^{\infty} \sum_{k_{1}=0}^{\infty} \sum_{l_{1}=0}^{\infty} \sum_{m_{1}=0}^{\infty} k_{1} P_{i_{1} j_{1} k_{1} l_{1} m_{1}} \\
& +k_{t r t} \sum_{i=1}^{\infty} \sum_{j=0}^{\infty} \sum_{k=0}^{\infty} \sum_{l=1}^{\infty} \sum_{m=0}^{\infty} i^{2}(k+1) P_{i j k+1 l-1 m} \sum_{i_{1}=1}^{\infty} \sum_{j_{1}=0}^{\infty} \sum_{k_{1}=0}^{\infty} \sum_{l_{1}=0}^{\infty} \sum_{m_{1}=0}^{\infty} l_{1} P_{i_{1} j_{1} k_{1} l_{1} m_{1}} \\
& +k_{t r t} \sum_{i=1}^{\infty} \sum_{j=0}^{\infty} \sum_{k=0}^{\infty} \sum_{l=0}^{\infty} \sum_{m=1}^{\infty} i^{2}(l+1) P_{i j k l+1 m-1} \sum_{i_{1}=1}^{\infty} \sum_{j_{1}=0}^{\infty} \sum_{k_{1}=0}^{\infty} \sum_{l_{1}=0}^{\infty} \sum_{m_{1}=0}^{\infty} k_{1} P_{i_{1} j_{1} k_{1} l_{1} m_{1}} \\
& -k_{t r p} \sum_{i=1}^{\infty} \sum_{j=0}^{\infty} \sum_{k=0}^{\infty} \sum_{l=0}^{\infty} \sum_{m=0}^{\infty} i^{2} k P_{i j k l m} \sum_{i_{1}=1}^{\infty} \sum_{j_{1}=0}^{\infty} \sum_{k_{1}=0}^{\infty} \sum_{l_{1}=0}^{\infty} \sum_{m_{1}=0}^{\infty} i_{1} P_{i_{1} j_{1} k_{1} l_{1} m_{1}} \\
& -k_{\operatorname{trp}} \sum_{i=1}^{\infty} \sum_{j=0}^{\infty} \sum_{k=0}^{\infty} \sum_{l=0}^{\infty} \sum_{m=0}^{\infty} i^{3} P_{i j k l m} \sum_{i_{1}=1}^{\infty} \sum_{j_{1}=0}^{\infty} \sum_{k_{1}=0}^{\infty} \sum_{l_{1}=0}^{\infty} \sum_{m_{1}=0}^{\infty} k_{1} P_{i_{1} j_{1} k_{1} l_{1} m_{1}} \\
& +k_{\operatorname{trp}} \sum_{i=1}^{\infty} \sum_{j=0}^{\infty} \sum_{k=0}^{\infty} \sum_{l=0}^{\infty} \sum_{m=0}^{\infty} i^{2}(k+1) P_{i j k+1 l m} \sum_{i_{1}=1}^{\infty} \sum_{j_{1}=0}^{\infty} \sum_{k_{1}=0}^{\infty} \sum_{l_{1}=0}^{\infty} \sum_{m_{1}=0}^{\infty} i_{1} P_{i_{1} j_{1} k_{1} l_{1} m_{1}} \\
& +k_{t r p} \sum_{i=1}^{\infty} \sum_{j=1}^{\infty} \sum_{k=1}^{\infty} \sum_{l=0}^{\infty} \sum_{m=0}^{\infty} i^{3} P_{i j-1 k-1 l m} \sum_{i_{1}=1}^{\infty} \sum_{j_{1}=0}^{\infty} \sum_{k_{1}=0}^{\infty} \sum_{l_{1}=0}^{\infty} \sum_{m_{1}=0}^{\infty} k_{1} P_{i_{1} j_{1} k_{1} l_{1} m_{1}}
\end{aligned}
$$




$$
\begin{aligned}
& -k_{t r p} \sum_{i=1}^{\infty} \sum_{j=0}^{\infty} \sum_{k=0}^{\infty} \sum_{l=0}^{\infty} \sum_{m=0}^{\infty} i^{2} m P_{i j k l m} \sum_{i_{1}=1}^{\infty} \sum_{j_{1}=0}^{\infty} \sum_{k_{1}=0}^{\infty} \sum_{l_{1}=0}^{\infty} \sum_{m_{1}=0}^{\infty} i_{1} P_{i_{1} j_{1} k_{1} l_{1} m_{1}} \\
& -k_{\text {trp }} \sum_{i=1}^{\infty} \sum_{j=0}^{\infty} \sum_{k=0}^{\infty} \sum_{l=0}^{\infty} \sum_{m=0}^{\infty} i^{3} P_{i j k l m} \sum_{i_{1}=1}^{\infty} \sum_{j_{1}=0}^{\infty} \sum_{k_{1}=0}^{\infty} \sum_{l_{1}=0}^{\infty} \sum_{m_{1}=0}^{\infty} m_{1} P_{i_{1} j_{1} k_{1} l_{1} m_{1}} \\
& +k_{\operatorname{trp}} \sum_{i=1}^{\infty} \sum_{j=0}^{\infty} \sum_{k=0}^{\infty} \sum_{l=0}^{\infty} \sum_{m=0}^{\infty} i^{2}(m+1) P_{i j k l m+1} \sum_{i_{1}=1}^{\infty} \sum_{j_{1}=0}^{\infty} \sum_{k_{1}=0}^{\infty} \sum_{l_{1}=0}^{\infty} \sum_{m_{1}=0}^{\infty} i_{1} P_{i_{1} j_{1} k_{1} l_{1} m_{1}} \\
& +k_{\operatorname{trp}} \sum_{i=1}^{\infty} \sum_{j=1}^{\infty} \sum_{k=0}^{\infty} \sum_{l=0}^{\infty} \sum_{m=1}^{\infty} i^{3} P_{i j-1 k l m-1} \sum_{i_{1}=1}^{\infty} \sum_{j_{1}=0}^{\infty} \sum_{k_{1}=0}^{\infty} \sum_{l_{1}=0}^{\infty} \sum_{m_{1}=0}^{\infty} m_{1} P_{i_{1} j_{1} k_{1} l_{1} m_{1}} \\
& -k_{\text {intr }} \sum_{i=1}^{\infty} \sum_{j=0}^{\infty} \sum_{k=0}^{\infty} \sum_{l=0}^{\infty} \sum_{m=0}^{\infty} i^{2} k P_{i j k l m} \\
& +k_{i n t r} \sum_{i=1}^{\infty} \sum_{j=1}^{\infty} \sum_{k=0}^{\infty} \sum_{l=0}^{\infty} \sum_{m=0}^{\infty} i^{2} k P_{i j-1 k l m} \\
& -k_{r} \sum_{i=1}^{\infty} \sum_{j=0}^{\infty} \sum_{k=0}^{\infty} \sum_{l=0}^{\infty} \sum_{m=0}^{\infty} i^{2} m P_{i j k l m} \\
& +k_{r} \sum_{i=1}^{\infty} \sum_{j=0}^{\infty} \sum_{k=1}^{\infty} \sum_{l=0}^{\infty} \sum_{m=0}^{\infty} i^{2}(m+1) P_{i j k-1 l m+1} \\
& -k_{t c} \sum_{i=1}^{\infty} \sum_{j=0}^{\infty} \sum_{k=0}^{\infty} \sum_{l=0}^{\infty} \sum_{m=0}^{\infty} i^{2} k P_{i j k l m} \sum_{i_{1}=1}^{\infty} \sum_{j_{1}=0}^{\infty} \sum_{k_{1}=0}^{\infty} \sum_{l_{1}=0}^{\infty} \sum_{m_{1}=0}^{\infty} k_{1} P_{i_{1} j_{1} k_{1} l_{1} m_{1}} \\
& +\frac{1}{2} k_{t c} \sum_{i=1}^{\infty} \sum_{j=0}^{\infty} \sum_{k=0}^{\infty} \sum_{l=0}^{\infty} \sum_{m=0}^{\infty} \sum_{i_{1}=1}^{i} \sum_{j_{1}=0}^{j} \sum_{k_{1}=0}^{k+2} \sum_{l_{1}=0}^{l} \sum_{m_{1}=0}^{m}\left[i^{2} k_{1} P_{i_{1} j_{1} k_{1} l_{1} m_{1}}\left(k+2-k_{1}\right) P_{i-i_{1}, j-j_{1}, k+2-k_{1}, l-l_{1} m-m_{1}}\right] \\
& -k_{t c} \sum_{i=1}^{\infty} \sum_{j=0}^{\infty} \sum_{k=0}^{\infty} \sum_{l=0}^{\infty} \sum_{m=0}^{\infty} i^{2} m P_{i j k l m} \sum_{i_{1}=1}^{\infty} \sum_{j_{1}=0}^{\infty} \sum_{k_{1}=0}^{\infty} \sum_{l_{1}=0}^{\infty} \sum_{m_{1}=0}^{\infty} m_{1} P_{i_{1} j_{1} k_{1} l_{1} m_{1}} \\
& +\frac{1}{2} k_{t c} \sum_{i=1}^{\infty} \sum_{j=0}^{\infty} \sum_{k=0}^{\infty} \sum_{l=0}^{\infty} \sum_{m=0}^{\infty} \sum_{i_{1}=1}^{i} \sum_{j_{1}=0}^{j} \sum_{k_{1}=0}^{k} \sum_{l_{1}=0}^{l} \sum_{m_{1}=0}^{m+2}\left[i^{2} m_{1} P_{i_{1} j_{1} k_{1} l_{1} m_{1}}\left(m+2-m_{1}\right) P_{i-i_{1}, j-j_{1}, k-k_{1}, l-l_{1} m+2-m_{1}}\right] \\
& -k_{t c} \sum_{i=1}^{\infty} \sum_{j=0}^{\infty} \sum_{k=0}^{\infty} \sum_{l=0}^{\infty} \sum_{m=0}^{\infty} i^{2} k P_{i j k l m} \sum_{i_{1}=1}^{\infty} \sum_{j_{1}=0}^{\infty} \sum_{k_{1}=0}^{\infty} \sum_{l_{1}=0}^{\infty} \sum_{m_{1}=0}^{\infty} m_{1} P_{i_{1} j_{1} k_{1} l_{1} m_{1}} \\
& -k_{t c} \sum_{i=1}^{\infty} \sum_{j=0}^{\infty} \sum_{k=0}^{\infty} \sum_{l=0}^{\infty} \sum_{m=0}^{\infty} i^{2} m P_{i j k l m} \sum_{i_{1}=1}^{\infty} \sum_{j_{1}=0}^{\infty} \sum_{k_{1}=0}^{\infty} \sum_{l_{1}=0}^{\infty} \sum_{m_{1}=0}^{\infty} k_{1} P_{i_{1} j_{1} k_{1} l_{1} m_{1}} \\
& +\frac{1}{2} k_{t c} \sum_{i=1}^{\infty} \sum_{j=0}^{\infty} \sum_{k=0}^{\infty} \sum_{l=0}^{\infty} \sum_{m=0}^{\infty} \sum_{i_{1}=1}^{i} \sum_{j_{1}=0}^{j} \sum_{k_{1}=0}^{k+1} \sum_{l_{1}=0}^{l} \sum_{m_{1}=0}^{m+1}\left[i^{2} k_{1} P_{i_{1} j_{1} k_{1} l_{1} m_{1}}\left(m+1-m_{1}\right) P_{\left.i-i_{1}, j-j_{1}, k+1-k_{1}, l-l_{1} m+1-m_{1}\right]}\right. \\
& +\frac{1}{2} k_{t c} \sum_{i=1}^{\infty} \sum_{j=0}^{\infty} \sum_{k=0}^{\infty} \sum_{l=0}^{\infty} \sum_{m=0}^{\infty} \sum_{i_{1}=1}^{i} \sum_{j_{1}=0}^{j} \sum_{k_{1}=0}^{k+1} \sum_{l_{1}=0}^{l} \sum_{m_{1}=0}^{m+1}\left[i^{2} m_{1} P_{i_{1} j_{1} k_{1} l_{1} m_{1}}\left(k+1-k_{1}\right) P_{i-i_{1}, j-j_{1}, k+1-k_{1}, l-l_{1} m+1-m_{1}}\right]
\end{aligned}
$$


Now

$$
\begin{aligned}
\sum_{i=1}^{\infty} \sum_{i_{1}=1}^{i} & \sum_{k=0}^{\infty} \sum_{k_{1}=0}^{k+2} i^{2} k_{1} P_{i_{1}, k_{1}}\left(k+2-k_{1}\right) P_{i-i_{1}, k+2-k_{1}} \\
= & \sum_{i=1}^{\infty} \sum_{i_{1}=1}^{i} \sum_{k=0}^{\infty} \sum_{k_{1}=0}^{k+2}\left[i_{1}+\left(i-i_{1}\right)\right]^{2} k_{1} P_{i_{1}, k_{1}}\left(k+2-k_{1}\right) P_{i-i_{1}, k+2-k_{1}} \\
= & \sum_{a=0}^{2}\left(\begin{array}{l}
2 \\
a
\end{array}\right) \sum_{i=1}^{\infty} \sum_{i_{1}=1}^{i} \sum_{k=0}^{\infty} \sum_{k_{1}=0}^{k+2} i_{1}^{a} k_{1} P_{i_{1}, k_{1}}\left(i-i_{1}\right)^{2-a}\left(k+2-k_{1}\right) P_{i-i_{1}, k+2-k_{1}} \\
= & \sum_{a=0}^{2}\left(\begin{array}{l}
2 \\
a
\end{array}\right) \sum_{i=1}^{\infty} \sum_{k=0}^{\infty} i^{a} k P_{i, k} \sum_{i_{1}=1}^{\infty} \sum_{k_{1}=0}^{\infty} i_{1}^{2-a} k_{1} P_{i_{1}, k_{1}} \\
= & \sum_{a=0}^{2}\left(\begin{array}{l}
2 \\
a
\end{array}\right) \mu_{a, 1} \mu_{2-a, 1} \\
= & \left(\frac{2 !}{0 ! 2 !} \mu_{01} \mu_{21}\right)+\left(\frac{2 !}{1 ! 1 !} \mu_{11} \mu_{11}\right)+\left(\frac{2 !}{2 ! 0 !} \mu_{21} \mu_{01}\right) \\
= & 2\left(\mu_{01} \mu_{21}+\mu_{11}^{2}\right)
\end{aligned}
$$

Similarly,

$$
\begin{aligned}
\sum_{i=1}^{\infty} \sum_{i_{1}=1}^{i} & \sum_{k=0}^{\infty} \sum_{k_{1}=0}^{k+1} \sum_{m=0}^{\infty} \sum_{m_{1}=0}^{m+1} i^{2} k_{1} P_{i_{1}, k_{1}, m_{1}}\left(m+1-m_{1}\right) P_{i-i_{1}, k+1-k_{1}, m+1-m_{1}} \\
& =\sum_{i=1}^{\infty} \sum_{i_{1}=1}^{i} \sum_{k=0}^{\infty} \sum_{k_{1}=0}^{k+1} \sum_{m=0}^{\infty} \sum_{m_{1}=0}^{m+1}\left[i_{1}+\left(i-i_{1}\right)\right]^{2} k_{1} P_{i_{1}, k_{1}, m_{1}}\left(m+1-m_{1}\right) P_{i-i_{1}, k+1-k_{1}, m+1-m_{1}} \\
& =\sum_{a=0}^{2}\left(\begin{array}{l}
2 \\
a
\end{array}\right) \sum_{i=1}^{\infty} \sum_{i_{1}=1}^{i} \sum_{k=0}^{\infty} \sum_{k_{1}=0}^{k+1} \sum_{m=0}^{\infty} \sum_{m_{1}=0}^{m+1} i_{1}^{a} k_{1} P_{i_{1}, k_{1}, m_{1}}\left(i-i_{1}\right)^{2-a}\left(m+1-m_{1}\right) P_{i-i_{1}, k+1-k_{1}, m+1-m_{1}} \\
& =\sum_{a=0}^{2}\left(\begin{array}{l}
2 \\
a
\end{array}\right) \sum_{i=1}^{\infty} \sum_{k=0}^{\infty} \sum_{m=0}^{\infty} i^{a} k P_{i, k, m} \sum_{i_{1}=1}^{\infty} \sum_{k_{1}=0}^{\infty} \sum_{m_{1}=0}^{\infty} i_{1}^{2-a} m_{1} P_{i_{1}, k_{1}, m_{1}} \\
& =\sum_{a=0}^{2}\left(\begin{array}{l}
2 \\
a
\end{array}\right) \mu_{a, 1,0} \mu_{2-a, 0,1} \\
= & \left(\frac{2 !}{0 ! 2 !} \mu_{010} \mu_{201}\right)+\left(\frac{2 !}{1 ! 1 !} \mu_{110} \mu_{101}\right)+\left(\frac{2 !}{2 ! 0 !} \mu_{210} \mu_{001}\right) \\
= & \mu_{010} \mu_{201}+\mu_{001} \mu_{210}+2 \mu_{110} \mu_{101}
\end{aligned}
$$




$$
\begin{aligned}
& \Rightarrow \frac{d \mu_{20000}}{d t}=k_{i} C_{M} C_{R} \\
& -k_{p} C_{M} \sum_{i=1}^{\infty} \sum_{j=0}^{\infty} \sum_{k=0}^{\infty} \sum_{l=0}^{\infty} \sum_{m=0}^{\infty} i^{2} k P_{i j k l m} \\
& +k_{p} C_{M} \sum_{i=1}^{\infty} \sum_{j=0}^{\infty} \sum_{k=0}^{\infty} \sum_{l=0}^{\infty} \sum_{m=0}^{\infty}(i+1)^{2} k P_{i j k l m} \\
& -k_{p} C_{M} \sum_{i=1}^{\infty} \sum_{j=0}^{\infty} \sum_{k=0}^{\infty} \sum_{l=0}^{\infty} \sum_{m=0}^{\infty} i^{2} m P_{i j k l m} \\
& +k_{p} C_{M} \sum_{i=1}^{\infty} \sum_{j=0}^{\infty} \sum_{k=0}^{\infty} \sum_{l=0}^{\infty} \sum_{m=0}^{\infty}(i+1)^{2} m P_{i j k l m} \\
& -k_{t r t} C_{T} \sum_{i=1}^{\infty} \sum_{j=0}^{\infty} \sum_{k=0}^{\infty} \sum_{l=0}^{\infty} \sum_{m=0}^{\infty} i^{2} k P_{i j k l m} \\
& +k_{t r t} C_{T} \sum_{i=1}^{\infty} \sum_{j=0}^{\infty} \sum_{k=0}^{\infty} \sum_{l=0}^{\infty} \sum_{m=0}^{\infty} i^{2}(k) P_{i j k l m} \\
& -k_{t r t} \sum_{i=1}^{\infty} \sum_{j=0}^{\infty} \sum_{k=0}^{\infty} \sum_{l=0}^{\infty} \sum_{m=0}^{\infty} i^{2} k P_{i j k l m} \sum_{i_{1}=1}^{\infty} \sum_{j_{1}=0}^{\infty} \sum_{k_{1}=0}^{\infty} \sum_{l_{1}=0}^{\infty} \sum_{m_{1}=0}^{\infty} l_{1} P_{i_{1} j_{1} k_{1} l_{1} m_{1}} \\
& -k_{t r t} \sum_{i=1}^{\infty} \sum_{j=0}^{\infty} \sum_{k=0}^{\infty} \sum_{l=0}^{\infty} \sum_{m=0}^{\infty} i^{2} l P_{i j k l m} \sum_{i_{1}=1}^{\infty} \sum_{j_{1}=0}^{\infty} \sum_{k_{1}=0}^{\infty} \sum_{l_{1}=0}^{\infty} \sum_{m_{1}=0}^{\infty} k_{1} P_{i_{1} j_{1} k_{1} l_{1} m_{1}} \\
& +k_{t r t} \sum_{i=1}^{\infty} \sum_{j=0}^{\infty} \sum_{k=0}^{\infty} \sum_{l=0}^{\infty} \sum_{m=0}^{\infty} i^{2} k P_{i j k l m} \sum_{i_{1}=1}^{\infty} \sum_{j_{1}=0}^{\infty} \sum_{k_{1}=0}^{\infty} \sum_{l_{1}=0}^{\infty} \sum_{m_{1}=0}^{\infty} l_{1} P_{i_{1} j_{1} k_{1} l_{1} m_{1}} \\
& +k_{t r t} \sum_{i=1}^{\infty} \sum_{j=0}^{\infty} \sum_{k=0}^{\infty} \sum_{l=0}^{\infty} \sum_{m=0}^{\infty} i^{2} l P_{i j k l m} \sum_{i_{1}=1}^{\infty} \sum_{j_{1}=0}^{\infty} \sum_{k_{1}=0}^{\infty} \sum_{l_{1}=0}^{\infty} \sum_{m_{1}=0}^{\infty} k_{1} P_{i_{1} j_{1} k_{1} l_{1} m_{1}} \\
& -k_{\text {trp }} \sum_{i=1}^{\infty} \sum_{j=0}^{\infty} \sum_{k=0}^{\infty} \sum_{l=0}^{\infty} \sum_{m=0}^{\infty} i^{2} k P_{i j k l m} \sum_{i_{1}=1}^{\infty} \sum_{j_{1}=0}^{\infty} \sum_{k_{1}=0}^{\infty} \sum_{l_{1}=0}^{\infty} \sum_{m_{1}=0}^{\infty} i_{1} P_{i_{1} j_{1} k_{1} l_{1} m_{1}} \\
& -k_{\text {trp }} \sum_{i=1}^{\infty} \sum_{j=0}^{\infty} \sum_{k=0}^{\infty} \sum_{l=0}^{\infty} \sum_{m=0}^{\infty} i^{3} P_{i j k l m} \sum_{i_{1}=1}^{\infty} \sum_{j_{1}=0}^{\infty} \sum_{k_{1}=0}^{\infty} \sum_{l_{1}=0}^{\infty} \sum_{m_{1}=0}^{\infty} k_{1} P_{i_{1} j_{1} k_{1} l_{1} m_{1}} \\
& +k_{t r p} \sum_{i=1}^{\infty} \sum_{j=0}^{\infty} \sum_{k=0}^{\infty} \sum_{l=0}^{\infty} \sum_{m=0}^{\infty} i^{2} k P_{i j k l m} \sum_{i_{1}=1}^{\infty} \sum_{j_{1}=0}^{\infty} \sum_{k_{1}=0}^{\infty} \sum_{l_{1}=0}^{\infty} \sum_{m_{1}=0}^{\infty} i_{1} P_{i_{1} j_{1} k_{1} l_{1} m_{1}} \\
& +k_{t r p} \sum_{i=1}^{\infty} \sum_{j=0}^{\infty} \sum_{k=0}^{\infty} \sum_{l=0}^{\infty} \sum_{m=0}^{\infty} i^{3} P_{i j k l m} \sum_{i_{1}=1}^{\infty} \sum_{j_{1}=0}^{\infty} \sum_{k_{1}=0}^{\infty} \sum_{l_{1}=0}^{\infty} \sum_{m_{1}=0}^{\infty} k_{1} P_{i_{1} j_{1} k_{1} l_{1} m_{1}}
\end{aligned}
$$




$$
\begin{aligned}
& -k_{\operatorname{trp}} \sum_{i=1}^{\infty} \sum_{j=0}^{\infty} \sum_{k=0}^{\infty} \sum_{l=0}^{\infty} \sum_{m=0}^{\infty} i^{2} m P_{i j k l m} \sum_{i_{1}=1}^{\infty} \sum_{j_{1}=0}^{\infty} \sum_{k_{1}=0}^{\infty} \sum_{l_{1}=0}^{\infty} \sum_{m_{1}=0}^{\infty} i_{1} P_{i_{1} j_{1} k_{1} l_{1} m_{1}} \\
& -k_{\operatorname{trp}} \sum_{i=1}^{\infty} \sum_{j=0}^{\infty} \sum_{k=0}^{\infty} \sum_{l=0}^{\infty} \sum_{m=0}^{\infty} i^{3} P_{i j k l m} \sum_{i_{1}=1}^{\infty} \sum_{j_{1}=0}^{\infty} \sum_{k_{1}=0}^{\infty} \sum_{l_{1}=0}^{\infty} \sum_{m_{1}=0}^{\infty} m_{1} P_{i_{1} j_{1} k_{1} l_{1} m_{1}} \\
& +k_{\text {trp }} \sum_{i=1}^{\infty} \sum_{j=0}^{\infty} \sum_{k=0}^{\infty} \sum_{l=0}^{\infty} \sum_{m=0}^{\infty} i^{2} m P_{i j k l m} \sum_{i_{1}=1}^{\infty} \sum_{j_{1}=0}^{\infty} \sum_{k_{1}=0}^{\infty} \sum_{l_{1}=0}^{\infty} \sum_{m_{1}=0}^{\infty} i_{1} P_{i_{1} j_{1} k_{1} l_{1} m_{1}} \\
& +k_{\text {trp }} \sum_{i=1}^{\infty} \sum_{j=0}^{\infty} \sum_{k=0}^{\infty} \sum_{l=0}^{\infty} \sum_{m=0}^{\infty} i^{3} P_{i j k l m} \sum_{i_{1}=1}^{\infty} \sum_{j_{1}=0}^{\infty} \sum_{k_{1}=0}^{\infty} \sum_{l_{1}=0}^{\infty} \sum_{m_{1}=0}^{\infty} m_{1} P_{i_{1} j_{1} k_{1} l_{1} m_{1}} \\
& -k_{\text {intr }} \sum_{i=1}^{\infty} \sum_{j=0}^{\infty} \sum_{k=0}^{\infty} \sum_{l=0}^{\infty} \sum_{m=0}^{\infty} i^{2} k P_{i j k l m} \\
& +k_{\text {intr }} \sum_{i=1}^{\infty} \sum_{j=0}^{\infty} \sum_{k=0}^{\infty} \sum_{l=0}^{\infty} \sum_{m=0}^{\infty} i^{2} k P_{i j k l m} \\
& -k_{r} \sum_{i=1}^{\infty} \sum_{j=0}^{\infty} \sum_{k=0}^{\infty} \sum_{l=0}^{\infty} \sum_{m=0}^{\infty} i^{2} m P_{i j k l m} \\
& +k_{r} \sum_{i=1}^{\infty} \sum_{j=0}^{\infty} \sum_{k=0}^{\infty} \sum_{l=0}^{\infty} \sum_{m=0}^{\infty} i^{2} m P_{i j k l m} \\
& -k_{t c} \sum_{i=1}^{\infty} \sum_{j=0}^{\infty} \sum_{k=0}^{\infty} \sum_{l=0}^{\infty} \sum_{m=0}^{\infty} i^{2} k P_{i j k l m} \sum_{i_{1}=1}^{\infty} \sum_{j_{1}=0}^{\infty} \sum_{k_{1}=0}^{\infty} \sum_{l_{1}=0}^{\infty} \sum_{m_{1}=0}^{\infty} k_{1} P_{i_{1} j_{1} k_{1} l_{1} m_{1}} \\
& +\frac{1}{2}(2) k_{t c} \sum_{i=1}^{\infty} \sum_{j=0}^{\infty} \sum_{k=0}^{\infty} \sum_{l=0}^{\infty} \sum_{m=0}^{\infty} i^{2} k P_{i j k l m} \sum_{i_{1}=1}^{\infty} \sum_{j_{1}=0}^{\infty} \sum_{k_{1}=0}^{\infty} \sum_{l_{1}=0}^{\infty} \sum_{m_{1}=0}^{\infty} k_{1} P_{i_{1} j_{1} k_{1} l_{1} m_{1}} \\
& +\frac{1}{2}(2) k_{t c} \sum_{i=1}^{\infty} \sum_{j=0}^{\infty} \sum_{k=0}^{\infty} \sum_{l=0}^{\infty} \sum_{m=0}^{\infty} i k P_{i j k l m} \sum_{i_{1}=1}^{\infty} \sum_{j_{1}=0}^{\infty} \sum_{k_{1}=0}^{\infty} \sum_{l_{1}=0}^{\infty} \sum_{m_{1}=0}^{\infty} i_{1} k_{1} P_{i_{1} j_{1} k_{1} l_{1} m_{1}} \\
& -k_{t c} \sum_{i=1}^{\infty} \sum_{j=0}^{\infty} \sum_{k=0}^{\infty} \sum_{l=0}^{\infty} \sum_{m=0}^{\infty} i^{2} m P_{i j k l m} \sum_{i_{1}=1}^{\infty} \sum_{j_{1}=0}^{\infty} \sum_{k_{1}=0}^{\infty} \sum_{l_{1}=0}^{\infty} \sum_{m_{1}=0}^{\infty} m_{1} P_{i_{1} j_{1} k_{1} l_{1} m_{1}} \\
& +\frac{1}{2}(2) k_{t c} \sum_{i=1}^{\infty} \sum_{j=0}^{\infty} \sum_{k=0}^{\infty} \sum_{l=0}^{\infty} \sum_{m=0}^{\infty} i^{2} m P_{i j k l m} \sum_{i_{1}=1}^{\infty} \sum_{j_{1}=0}^{\infty} \sum_{k_{1}=0}^{\infty} \sum_{l_{1}=0}^{\infty} \sum_{m_{1}=0}^{\infty} m_{1} P_{i_{1} j_{1} k_{1} l_{1} m_{1}} \\
& +\frac{1}{2}(2) k_{t c} \sum_{i=1}^{\infty} \sum_{j=0}^{\infty} \sum_{k=0}^{\infty} \sum_{l=0}^{\infty} \sum_{m=0}^{\infty} i m P_{i j k l m} \sum_{i_{1}=1}^{\infty} \sum_{j_{1}=0}^{\infty} \sum_{k_{1}=0}^{\infty} \sum_{l_{1}=0}^{\infty} \sum_{m_{1}=0}^{\infty} i_{1} m_{1} P_{i_{1} j_{1} k_{1} l_{1} m_{1}} \\
& -k_{t c} \sum_{i=1}^{\infty} \sum_{j=0}^{\infty} \sum_{k=0}^{\infty} \sum_{l=0}^{\infty} \sum_{m=0}^{\infty} i^{2} k P_{i j k l m} \sum_{i_{1}=1}^{\infty} \sum_{j_{1}=0}^{\infty} \sum_{k_{1}=0}^{\infty} \sum_{l_{1}=0}^{\infty} \sum_{m_{1}=0}^{\infty} m_{1} P_{i_{1} j_{1} k_{1} l_{1} m_{1}} \\
& -k_{t c} \sum_{i=1}^{\infty} \sum_{j=0}^{\infty} \sum_{k=0}^{\infty} \sum_{l=0}^{\infty} \sum_{m=0}^{\infty} i^{2} m P_{i j k l m} \sum_{i_{1}=1}^{\infty} \sum_{j_{1}=0}^{\infty} \sum_{k_{1}=0}^{\infty} \sum_{l_{1}=0}^{\infty} \sum_{m_{1}=0}^{\infty} k_{1} P_{i_{1} j_{1} k_{1} l_{1} m_{1}}
\end{aligned}
$$




$$
\begin{aligned}
& +\frac{1}{2}(2) k_{t c} \sum_{i=1}^{\infty} \sum_{j=0}^{\infty} \sum_{k=0}^{\infty} \sum_{l=0}^{\infty} \sum_{m=0}^{\infty} i^{2} k P_{i j k l m} \sum_{i_{1}=1}^{\infty} \sum_{j_{1}=0}^{\infty} \sum_{k_{1}=0}^{\infty} \sum_{l_{1}=0}^{\infty} \sum_{m_{1}=0}^{\infty} m_{1} P_{i_{1} j_{1} k_{1} l_{1} m_{1}} \\
& +\frac{1}{2}(2) k_{t c} \sum_{i=1}^{\infty} \sum_{j=0}^{\infty} \sum_{k=0}^{\infty} \sum_{l=0}^{\infty} \sum_{m=0}^{\infty} i^{2} m P_{i j k l m} \sum_{i_{1}=1}^{\infty} \sum_{j_{1}=0}^{\infty} \sum_{k_{1}=0}^{\infty} \sum_{l_{1}=0}^{\infty} \sum_{m_{1}=0}^{\infty} k_{1} P_{i_{1} j_{1} k_{1} l_{1} m_{1}} \\
& +\frac{1}{2}(4) k_{t c} \sum_{i=1}^{\infty} \sum_{j=0}^{\infty} \sum_{k=0}^{\infty} \sum_{l=0}^{\infty} \sum_{m=0}^{\infty} i k P_{i j k l m} \sum_{i_{1}=1}^{\infty} \sum_{j_{1}=0}^{\infty} \sum_{k_{1}=0}^{\infty} \sum_{l_{1}=0}^{\infty} \sum_{m_{1}=0}^{\infty} i_{1} m_{1} P_{i_{1} j_{1} k_{1} l_{1} m_{1}}
\end{aligned}
$$

using Equations (67) and (68)

$$
\begin{aligned}
& \Rightarrow \frac{d \mu_{20000}}{d t}=k_{i} C_{M} C_{R} \\
& \quad+k_{p} C_{M} \sum_{i=1}^{\infty} \sum_{j=0}^{\infty} \sum_{k=0}^{\infty} \sum_{l=0}^{\infty} \sum_{m=0}^{\infty}(2 i+1) k P_{i j k l m} \\
& \quad+k_{p} C_{M} \sum_{i=1}^{\infty} \sum_{j=0}^{\infty} \sum_{k=0}^{\infty} \sum_{l=0}^{\infty} \sum_{m=0}^{\infty}(2 i+1) m P_{i j k l m} \\
& \quad+k_{t c} \sum_{i=1}^{\infty} \sum_{j=0}^{\infty} \sum_{k=0}^{\infty} \sum_{l=0}^{\infty} \sum_{m=0}^{\infty} i k P_{i j k l m} \sum_{i_{1}=1}^{\infty} \sum_{j_{1}=0}^{\infty} \sum_{k_{1}=0}^{\infty} \sum_{l_{1}=0}^{\infty} \sum_{m_{1}=0}^{\infty} i_{1} k_{1} P_{i_{1} j_{1} k_{1} l_{1} m_{1}} \\
& \quad+k_{t c} \sum_{i=1}^{\infty} \sum_{j=0}^{\infty} \sum_{k=0}^{\infty} \sum_{l=0}^{\infty} \sum_{m=0}^{\infty} i m P_{i j k l m} \sum_{i_{1}=1}^{\infty} \sum_{j_{1}=0}^{\infty} \sum_{k_{1}=0}^{\infty} \sum_{l_{1}=0}^{\infty} \sum_{m_{1}=0}^{\infty} i_{1} m_{1} P_{i_{1} j_{1} k_{1} l_{1} m_{1}} \\
& \quad+2 k_{t c} \sum_{i=1}^{\infty} \sum_{j=0}^{\infty} \sum_{k=0}^{\infty} \sum_{l=0}^{\infty} \sum_{m=0}^{\infty} i k P_{i j k l m} \sum_{i_{1}=1}^{\infty} \sum_{j_{1}=0}^{\infty} \sum_{k_{1}=0}^{\infty} \sum_{l_{1}=0}^{\infty} \sum_{m_{1}=0}^{\infty} i_{1} m_{1} P_{i_{1} j_{1} k_{1} l_{1} m_{1}} \\
& \Rightarrow \frac{d \mu_{20000}}{d t}=k_{i} C_{M} C_{R}+k_{p} C_{M}\left(2 \mu_{10100}+\mu_{00100}\right)+k_{p} C_{M}\left(2 \mu_{10001}+\mu_{00001}\right)+k_{t c}\left(\mu_{10100}\right)^{2} \\
& +k_{t c}\left(\mu_{10001}\right)^{2}+2 k_{t c} \mu_{10100} \mu_{10001}
\end{aligned}
$$


3.3.2 Second moment w.r.t. the number of repeat units and active (uncapped) radicals

$$
\begin{aligned}
& \frac{d \mu_{10100}}{d t}=\sum_{i=1}^{\infty} \sum_{j=0}^{\infty} \sum_{k=0}^{\infty} \sum_{l=0}^{\infty} \sum_{m=0}^{\infty} i k \frac{d P_{i j k l m}}{d t} \\
& =k_{i} C_{M} C_{R} \\
& -k_{p} C_{M} \sum_{i=1}^{\infty} \sum_{j=0}^{\infty} \sum_{k=0}^{\infty} \sum_{l=0}^{\infty} \sum_{m=0}^{\infty} i k^{2} P_{i j k l m} \\
& +k_{p} C_{M} \sum_{i=2}^{\infty} \sum_{j=0}^{\infty} \sum_{k=0}^{\infty} \sum_{l=0}^{\infty} \sum_{m=0}^{\infty} i k^{2} P_{i-1 j k l m} \\
& -k_{p} C_{M} \sum_{i=1}^{\infty} \sum_{j=0}^{\infty} \sum_{k=0}^{\infty} \sum_{l=0}^{\infty} \sum_{m=0}^{\infty} i k m P_{i j k l m} \\
& +k_{p} C_{M} \sum_{i=2}^{\infty} \sum_{j=0}^{\infty} \sum_{k=0}^{\infty} \sum_{l=0}^{\infty} \sum_{m=0}^{\infty} i k m P_{i-1 j k l m} \\
& -k_{t r t} C_{T} \sum_{i=1}^{\infty} \sum_{j=0}^{\infty} \sum_{k=0}^{\infty} \sum_{l=0}^{\infty} \sum_{m=0}^{\infty} i k^{2} P_{i j k l m} \\
& +k_{t r t} C_{T} \sum_{i=1}^{\infty} \sum_{j=0}^{\infty} \sum_{k=0}^{\infty} \sum_{l=1}^{\infty} \sum_{m=0}^{\infty} i k(k+1) P_{i j k+1 l-1 m} \\
& -k_{t r t} \sum_{i=1}^{\infty} \sum_{j=0}^{\infty} \sum_{k=0}^{\infty} \sum_{l=0}^{\infty} \sum_{m=0}^{\infty} i k^{2} P_{i j k l m} \sum_{i_{1}=1}^{\infty} \sum_{j_{1}=0}^{\infty} \sum_{k_{1}=0}^{\infty} \sum_{l_{1}=0}^{\infty} \sum_{m_{1}=0}^{\infty} l_{1} P_{i_{1} j_{1} k_{1} l_{1} m_{1}} \\
& -k_{t r t} \sum_{i=1}^{\infty} \sum_{j=0}^{\infty} \sum_{k=0}^{\infty} \sum_{l=0}^{\infty} \sum_{m=0}^{\infty} i k l P_{i j k l m} \sum_{i_{1}=1}^{\infty} \sum_{j_{1}=0}^{\infty} \sum_{k_{1}=0}^{\infty} \sum_{l_{1}=0}^{\infty} \sum_{m_{1}=0}^{\infty} k_{1} P_{i_{1} j_{1} k_{1} l_{1} m_{1}} \\
& +k_{t r t} \sum_{i=1}^{\infty} \sum_{j=0}^{\infty} \sum_{k=0}^{\infty} \sum_{l=1}^{\infty} \sum_{m=0}^{\infty} i k(k+1) P_{i j k+1 l-1 m} \sum_{i_{1}=1}^{\infty} \sum_{j_{1}=0}^{\infty} \sum_{k_{1}=0}^{\infty} \sum_{l_{1}=0}^{\infty} \sum_{m_{1}=0}^{\infty} l_{1} P_{i_{1} j_{1} k_{1} l_{1} m_{1}} \\
& +k_{t r t} \sum_{i=1}^{\infty} \sum_{j=0}^{\infty} \sum_{k=0}^{\infty} \sum_{l=0}^{\infty} \sum_{m=1}^{\infty} i k(l+1) P_{i j k l+1 m-1} \sum_{i_{1}=1}^{\infty} \sum_{j_{1}=0}^{\infty} \sum_{k_{1}=0}^{\infty} \sum_{l_{1}=0}^{\infty} \sum_{m_{1}=0}^{\infty} k_{1} P_{i_{1} j_{1} k_{1} l_{1} m_{1}} \\
& -k_{\operatorname{trp}} \sum_{i=1}^{\infty} \sum_{j=0}^{\infty} \sum_{k=0}^{\infty} \sum_{l=0}^{\infty} \sum_{m=0}^{\infty} i k^{2} P_{i j k l m} \sum_{i_{1}=1}^{\infty} \sum_{j_{1}=0}^{\infty} \sum_{k_{1}=0}^{\infty} \sum_{l_{1}=0}^{\infty} \sum_{m_{1}=0}^{\infty} i_{1} P_{i_{1} j_{1} k_{1} l_{1} m_{1}} \\
& -k_{\operatorname{trp}} \sum_{i=1}^{\infty} \sum_{j=0}^{\infty} \sum_{k=0}^{\infty} \sum_{l=0}^{\infty} \sum_{m=0}^{\infty} i^{2} k P_{i j k l m} \sum_{i_{1}=1}^{\infty} \sum_{j_{1}=0}^{\infty} \sum_{k_{1}=0}^{\infty} \sum_{l_{1}=0}^{\infty} \sum_{m_{1}=0}^{\infty} k_{1} P_{i_{1} j_{1} k_{1} l_{1} m_{1}} \\
& +k_{\operatorname{trp}} \sum_{i=1}^{\infty} \sum_{j=0}^{\infty} \sum_{k=0}^{\infty} \sum_{l=0}^{\infty} \sum_{m=0}^{\infty} i k(k+1) P_{i j k+1 l m} \sum_{i_{1}=1}^{\infty} \sum_{j_{1}=0}^{\infty} \sum_{k_{1}=0}^{\infty} \sum_{l_{1}=0}^{\infty} \sum_{m_{1}=0}^{\infty} i_{1} P_{i_{1} j_{1} k_{1} l_{1} m_{1}} \\
& +k_{t r p} \sum_{i=1}^{\infty} \sum_{j=1}^{\infty} \sum_{k=1}^{\infty} \sum_{l=0}^{\infty} \sum_{m=0}^{\infty} i^{2} k P_{i j-1 k-1 l m} \sum_{i_{1}=1}^{\infty} \sum_{j_{1}=0}^{\infty} \sum_{k_{1}=0}^{\infty} \sum_{l_{1}=0}^{\infty} \sum_{m_{1}=0}^{\infty} k_{1} P_{i_{1} j_{1} k_{1} l_{1} m_{1}}
\end{aligned}
$$


$-k_{t r p} \sum_{i=1}^{\infty} \sum_{j=0}^{\infty} \sum_{k=0}^{\infty} \sum_{l=0}^{\infty} \sum_{m=0}^{\infty} i k m P_{i j k l m} \sum_{i_{1}=1}^{\infty} \sum_{j_{1}=0}^{\infty} \sum_{k_{1}=0}^{\infty} \sum_{l_{1}=0}^{\infty} \sum_{m_{1}=0}^{\infty} i_{1} P_{i_{1} j_{1} k_{1} l_{1} m_{1}}$

$-k_{\text {trp }} \sum_{i=1}^{\infty} \sum_{j=0}^{\infty} \sum_{k=0}^{\infty} \sum_{l=0}^{\infty} \sum_{m=0}^{\infty} i^{2} k P_{i j k l m} \sum_{i_{1}=1}^{\infty} \sum_{j_{1}=0}^{\infty} \sum_{k_{1}=0}^{\infty} \sum_{l_{1}=0}^{\infty} \sum_{m_{1}=0}^{\infty} m_{1} P_{i_{1} j_{1} k_{1} l_{1} m_{1}}$

$+k_{t r p} \sum_{i=1}^{\infty} \sum_{j=0}^{\infty} \sum_{k=0}^{\infty} \sum_{l=0}^{\infty} \sum_{m=0}^{\infty} i k(m+1) P_{i j k l m+1} \sum_{i_{1}=1}^{\infty} \sum_{j_{1}=0}^{\infty} \sum_{k_{1}=0}^{\infty} \sum_{l_{1}=0}^{\infty} \sum_{m_{1}=0}^{\infty} i_{1} P_{i_{1} j_{1} k_{1} l_{1} m_{1}}$

$+k_{t r p} \sum_{i=1}^{\infty} \sum_{j=1}^{\infty} \sum_{k=0}^{\infty} \sum_{l=0}^{\infty} \sum_{m=1}^{\infty} i^{2} k P_{i j-1 k l m-1} \sum_{i_{1}=1}^{\infty} \sum_{j_{1}=0}^{\infty} \sum_{k_{1}=0}^{\infty} \sum_{l_{1}=0}^{\infty} \sum_{m_{1}=0}^{\infty} m_{1} P_{i_{1} j_{1} k_{1} l_{1} m_{1}}$

$-k_{i n t r} \sum_{i=1}^{\infty} \sum_{j=0}^{\infty} \sum_{k=0}^{\infty} \sum_{l=0}^{\infty} \sum_{m=0}^{\infty} i k^{2} P_{i j k l m}$

$+k_{\text {intr }} \sum_{i=1}^{\infty} \sum_{j=1}^{\infty} \sum_{k=0}^{\infty} \sum_{l=0}^{\infty} \sum_{m=0}^{\infty} i k^{2} P_{i j-1 k l m}$

$-k_{r} \sum_{i=1}^{\infty} \sum_{j=0}^{\infty} \sum_{k=0}^{\infty} \sum_{l=0}^{\infty} \sum_{m=0}^{\infty} i k m P_{i j k l m}$

$+k_{r} \sum_{i=1}^{\infty} \sum_{j=0}^{\infty} \sum_{k=1}^{\infty} \sum_{l=0}^{\infty} \sum_{m=0}^{\infty} i k(m+1) P_{i j k-1 l m+1}$

$-k_{t c} \sum_{i=1}^{\infty} \sum_{j=0}^{\infty} \sum_{k=0}^{\infty} \sum_{l=0}^{\infty} \sum_{m=0}^{\infty} i k^{2} P_{i j k l m} \sum_{i_{1}=1}^{\infty} \sum_{j_{1}=0}^{\infty} \sum_{k_{1}=0}^{\infty} \sum_{l_{1}=0}^{\infty} \sum_{m_{1}=0}^{\infty} k_{1} P_{i_{1} j_{1} k_{1} l_{1} m_{1}}$

$+\frac{1}{2} k_{t c} \sum_{i=1}^{\infty} \sum_{j=0}^{\infty} \sum_{k=0}^{\infty} \sum_{l=0}^{\infty} \sum_{m=0}^{\infty} \sum_{i_{1}=1}^{i} \sum_{j_{1}=0}^{j} \sum_{k_{1}=0}^{k+2} \sum_{l_{1}=0}^{l} \sum_{m_{1}=0}^{m}\left[i k k_{1} P_{i_{1} j_{1} k_{1} l_{1} m_{1}}\left(k+2-k_{1}\right) P_{i-i_{1}, j-j_{1}, k+2-k_{1}, l-l_{1} m-m_{1}}\right]$

$-k_{t c} \sum_{i=1}^{\infty} \sum_{j=0}^{\infty} \sum_{k=0}^{\infty} \sum_{l=0}^{\infty} \sum_{m=0}^{\infty} i k m P_{i j k l m} \sum_{i_{1}=1}^{\infty} \sum_{j_{1}=0}^{\infty} \sum_{k_{1}=0}^{\infty} \sum_{l_{1}=0}^{\infty} \sum_{m_{1}=0}^{\infty} m_{1} P_{i_{1} j_{1} k_{1} l_{1} m_{1}}$

$+\frac{1}{2} k_{t c} \sum_{i=1}^{\infty} \sum_{j=0}^{\infty} \sum_{k=0}^{\infty} \sum_{l=0}^{\infty} \sum_{m=0}^{\infty} \sum_{i_{1}=1}^{i} \sum_{j_{1}=0}^{j} \sum_{k_{1}=0}^{k} \sum_{l_{1}=0}^{l} \sum_{m_{1}=0}^{m+2}\left[i k m_{1} P_{i_{1} j_{1} k_{1} l_{1} m_{1}}\left(m+2-m_{1}\right) P_{i-i_{1}, j-j_{1}, k-k_{1}, l-l_{1} m+2-m_{1}}\right]$

$-k_{t c} \sum_{i=1}^{\infty} \sum_{j=0}^{\infty} \sum_{k=0}^{\infty} \sum_{l=0}^{\infty} \sum_{m=0}^{\infty} i k^{2} P_{i j k l m} \sum_{i_{1}=1}^{\infty} \sum_{j_{1}=0}^{\infty} \sum_{k_{1}=0}^{\infty} \sum_{l_{1}=0}^{\infty} \sum_{m_{1}=0}^{\infty} m_{1} P_{i_{1} j_{1} k_{1} l_{1} m_{1}}$

$-k_{t c} \sum_{i=1}^{\infty} \sum_{j=0}^{\infty} \sum_{k=0}^{\infty} \sum_{l=0}^{\infty} \sum_{m=0}^{\infty} i k m P_{i j k l m} \sum_{i_{1}=1}^{\infty} \sum_{j_{1}=0}^{\infty} \sum_{k_{1}=0}^{\infty} \sum_{l_{1}=0}^{\infty} \sum_{m_{1}=0}^{\infty} k_{1} P_{i_{1} j_{1} k_{1} l_{1} m_{1}}$

$+\frac{1}{2} k_{t c} \sum_{i=1}^{\infty} \sum_{j=0}^{\infty} \sum_{k=0}^{\infty} \sum_{l=0}^{\infty} \sum_{m=0}^{\infty} \sum_{i_{1}=1}^{i} \sum_{j_{1}=0}^{j} \sum_{k_{1}=0}^{k+1} \sum_{l_{1}=0}^{l} \sum_{m_{1}=0}^{m+1}\left[i k k_{1} P_{i_{1} j_{1} k_{1} l_{1} m_{1}}\left(m+1-m_{1}\right) P_{i-i_{1}, j-j_{1}, k+1-k_{1}, l-l_{1} m+1-m_{1}}\right]$

$+\frac{1}{2} k_{t c} \sum_{i=1}^{\infty} \sum_{j=0}^{\infty} \sum_{k=0}^{\infty} \sum_{l=0}^{\infty} \sum_{m=0}^{\infty} \sum_{i_{1}=1}^{i} \sum_{j_{1}=0}^{j} \sum_{k_{1}=0}^{k+1} \sum_{l_{1}=0}^{l} \sum_{m_{1}=0}^{m+1}\left[i k m_{1} P_{i_{1} j_{1} k_{1} l_{1} m_{1}}\left(k+1-k_{1}\right) P_{i-i_{1}, j-j_{1}, k+1-k_{1}, l-l_{1} m+1-m_{1}}\right]$ 
Now

$$
\begin{aligned}
\sum_{i=1}^{\infty} \sum_{i_{1}=1}^{i} & \sum_{k=0}^{\infty} \sum_{k_{1}=0}^{k+2} i k k_{1} P_{i_{1}, k_{1}}\left(k+2-k_{1}\right) P_{i-i_{1}, k+2-k_{1}} \\
= & \sum_{i=1}^{\infty} \sum_{i_{1}=1}^{i} \sum_{k=0}^{\infty} \sum_{k_{1}=0}^{k+2}\left[i_{1}+\left(i-i_{1}\right)\right]\left[k_{1}+\left(k-k_{1}\right)\right] k_{1} P_{i_{1}, k_{1}}\left(k+2-k_{1}\right) P_{i-i_{1}, k+2-k_{1}} \\
= & \sum_{a=0}^{1}\left(\begin{array}{l}
1 \\
a
\end{array}\right) \sum_{i=1}^{\infty} \sum_{i_{1}=1}^{i} \sum_{k=0}^{\infty} \sum_{k_{1}=0}^{k+2} i_{1}^{a} k_{1}^{1+a} P_{i_{1}, k_{1}}\left(i-i_{1}\right)^{1-a}\left(k+2-k_{1}-2\right)^{1-a}\left(k+2-k_{1}\right) P_{i-i_{1}, k+2-k_{1}} \\
= & \sum_{a=0}^{1}\left(\begin{array}{l}
1 \\
a
\end{array}\right) \sum_{i=1}^{\infty} \sum_{k=0}^{\infty} i^{a} k^{1+a} P_{i, k} \sum_{i_{1}=1}^{\infty} \sum_{k_{1}=0}^{\infty} i_{1}^{1-a}\left(k_{1}-2\right)^{1-a} k_{1} P_{i_{1}, k_{1}} \\
= & \sum_{a=0}^{1}\left(\begin{array}{l}
1 \\
a
\end{array}\right) \mu_{a, 1+a} \sum_{i_{1}=1}^{\infty} \sum_{k_{1}=0}^{\infty} i_{1}^{1-a}\left(k_{1}-2\right)^{1-a} k_{1} P_{i_{1}, k_{1}} \\
= & \left(\frac{1 !}{0 ! 1 !} \mu_{01} \sum_{i_{1}=1}^{\infty} \sum_{k_{1}=0}^{\infty} i_{1}\left(k_{1}-2\right) k_{1} P_{i_{1}, k_{1}}\right)+\left(\frac{1 !}{1 ! 0 !} \mu_{12} \sum_{i_{1}=1}^{\infty} \sum_{k_{1}=0}^{\infty} k_{1} P_{i_{1}, k_{1}}\right) \\
= & \mu_{01} \mu_{12}-2 \mu_{01} \mu_{11}+\mu_{12} \mu_{01}=2\left(\mu_{12} \mu_{01}-\mu_{01} \mu_{11}\right)
\end{aligned}
$$

$$
\begin{aligned}
\sum_{i=1}^{\infty} \sum_{i_{1}=1}^{i} & \sum_{k=0}^{\infty} \sum_{k_{1}=0}^{k} \sum_{m=0}^{\infty} \sum_{m_{1}=0}^{m+2} i k m_{1} P_{i_{1}, k_{1}, m_{1}}\left(m+2-m_{1}\right) P_{i-i_{1}, k-k_{1}, m+2-m_{1}} \\
& =\sum_{i=1}^{\infty} \sum_{i_{1}=1}^{i} \sum_{k=0}^{\infty} \sum_{k_{1}=0}^{k} \sum_{m=0}^{\infty} \sum_{m_{1}=0}^{m+2}\left[i_{1}+\left(i-i_{1}\right)\right]\left[k_{1}+\left(k-k_{1}\right)\right] m_{1} P_{i_{1}, k_{1}, m_{1}}\left(m+2-m_{1}\right) P_{i-i_{1}, k-k_{1}, m+2-m_{1}} \\
& =\sum_{a=0}^{1}\left(\begin{array}{l}
1 \\
a
\end{array}\right) \sum_{i=1}^{\infty} \sum_{i_{1}=1}^{i} \sum_{k=0}^{\infty} \sum_{k_{1}=0}^{k} \sum_{m=0}^{\infty} \sum_{m_{1}=0}^{m+2} i_{1}^{a} k_{1}^{a} m_{1} P_{i_{1}, k_{1}, m_{1}}\left(i-i_{1}\right)^{1-a}\left(k-k_{1}\right)^{1-a}\left(m+2-m_{1}\right) P_{i-i_{1}, k-k_{1}, m+2-m_{1}} \\
& =\sum_{a=0}^{1}\left(\begin{array}{l}
1 \\
a
\end{array}\right) \sum_{i=1}^{\infty} \sum_{k=0}^{\infty} \sum_{m=0}^{\infty} i^{a} k^{a} m P_{i, k, m} \sum_{i_{1}=1}^{\infty} \sum_{k_{1}=0}^{\infty} \sum_{m_{1}=0}^{\infty} i_{1}^{1-a} k_{1}^{1-a} m_{1} P_{i_{1}, k_{1}, m_{1}} \\
& =\sum_{a=0}^{1}\left(\begin{array}{l}
1 \\
a
\end{array}\right) \mu_{a, a, 1} \mu_{1-a .1-a, 1} \\
= & \left(\frac{1 !}{0 ! 1 !} \mu_{001} \mu_{111}\right)+\left(\frac{1 !}{1 ! 0 !} \mu_{111} \mu_{001}\right) \\
= & 2 \mu_{111} \mu_{001}
\end{aligned}
$$




$$
\begin{aligned}
& \sum_{i=1}^{\infty} \sum_{i_{1}=1}^{i} \sum_{k=0}^{\infty} \sum_{k_{1}=0}^{k+1} \sum_{m=0}^{\infty} \sum_{m_{1}=0}^{m+1} i k k_{1} P_{i_{1}, k_{1}, m_{1}}\left(m+1-m_{1}\right) P_{i-i_{1}, k+1-k_{1}, m+1-m_{1}} \\
& =\sum_{i=1}^{\infty} \sum_{i_{1}=1}^{i} \sum_{k=0}^{\infty} \sum_{k_{1}=0}^{k+1} \sum_{m=0}^{\infty} \sum_{m_{1}=0}^{m+1}\left[i_{1}+\left(i-i_{1}\right)\right]\left[k_{1}+\left(k-k_{1}\right)\right] k_{1} P_{i_{1}, k_{1}, m_{1}}\left(m+1-m_{1}\right) P_{i-i_{1}, k+1-k_{1}, m+1-m_{1}} \\
& =\sum_{a=0}^{1}\left(\begin{array}{l}
1 \\
a
\end{array}\right) \sum_{i=1}^{\infty} \sum_{i_{1}=1}^{i} \sum_{k=0}^{\infty} \sum_{k_{1}=0}^{k+1} \sum_{m=0}^{\infty} \sum_{m_{1}=0}^{m+1} i_{1}^{a} k_{1}^{1+a} P_{i_{1}, k_{1}, m_{1}} \\
& \times\left(i-i_{1}\right)^{1-a}\left(k+1-k_{1}-1\right)^{1-a}\left(m+1-m_{1}\right) P_{i-i_{1}, k+1-k_{1}, m+1-m_{1}} \\
& =\sum_{a=0}^{1}\left(\begin{array}{l}
1 \\
a
\end{array}\right) \sum_{i=1}^{\infty} \sum_{k=0}^{\infty} \sum_{m=0}^{\infty} i^{a} k^{1+a} P_{i, k, m} \sum_{i_{1}=1}^{\infty} \sum_{k_{1}=0}^{\infty} \sum_{m_{1}=0}^{\infty} i_{1}^{1-a}\left(k_{1}-1\right)^{1-a} m_{1} P_{i_{1}, k_{1}, m_{1}} \\
& =\sum_{a=0}^{1}\left(\begin{array}{l}
1 \\
a
\end{array}\right) \mu_{a, 1+a, 0} \sum_{i_{1}=1}^{\infty} \sum_{k_{1}=0}^{\infty} \sum_{m_{1}=0}^{\infty} i_{1}^{1-a}\left(k_{1}-1\right)^{1-a} m_{1} P_{i_{1}, k_{1}, m_{1}} \\
& =\left(\frac{1 !}{0 ! 1 !} \mu_{010} \sum_{i_{1}=1}^{\infty} \sum_{k_{1}=0}^{\infty} \sum_{m_{1}=0}^{\infty} i_{1}\left(k_{1}-1\right) m_{1} P_{i_{1}, k_{1}, m_{1}}\right)+\left(\frac{1 !}{1 ! 0 !} \mu_{120} \sum_{i_{1}=1}^{\infty} \sum_{k_{1}=0}^{\infty} \sum_{m_{1}=0}^{\infty} m_{1} P_{i_{1}, k_{1}, m_{1}}\right) \\
& =\mu_{010} \mu_{111}-\mu_{010} \mu_{101}+\mu_{120} \mu_{001} \\
& \sum_{i=1}^{\infty} \sum_{i_{1}=1}^{i} \sum_{k=0}^{\infty} \sum_{k_{1}=0}^{k+1} \sum_{m=0}^{\infty} \sum_{m_{1}=0}^{m+1} i k m_{1} P_{i_{1}, k_{1}, m_{1}}\left(k+1-k_{1}\right) P_{i-i_{1}, k+1-k_{1}, m+1-m_{1}} \\
& =\sum_{i=1}^{\infty} \sum_{i_{1}=1}^{i} \sum_{k=0}^{\infty} \sum_{k_{1}=0}^{k+1} \sum_{m=0}^{\infty} \sum_{m_{1}=0}^{m+1}\left[i_{1}+\left(i-i_{1}\right)\right]\left[k_{1}+\left(k-k_{1}\right)\right] m_{1} P_{i_{1}, k_{1}, m_{1}}\left(k+1-k_{1}\right) P_{i-i_{1}, k+1-k_{1}, m+1-m_{1}} \\
& =\sum_{a=0}^{1}\left(\begin{array}{l}
1 \\
a
\end{array}\right) \sum_{i=1}^{\infty} \sum_{i_{1}=1}^{i} \sum_{k=0}^{\infty} \sum_{k_{1}=0}^{k+1} \sum_{m=0}^{\infty} \sum_{m_{1}=0}^{m+1} i_{1}^{a} k_{1}^{a} m_{1} P_{i_{1}, k_{1}, m_{1}} \\
& \times\left(i-i_{1}\right)^{1-a}\left(k+1-k_{1}-1\right)^{1-a}\left(k+1-k_{1}\right) P_{i-i_{1}, k+1-k_{1}, m+1-m_{1}} \\
& =\sum_{a=0}^{1}\left(\begin{array}{l}
1 \\
a
\end{array}\right) \sum_{i=1}^{\infty} \sum_{k=0}^{\infty} \sum_{m=0}^{\infty} i^{a} k^{a} m_{1} P_{i, k, m} \sum_{i_{1}=1}^{\infty} \sum_{k_{1}=0}^{\infty} \sum_{m_{1}=0}^{\infty} i_{1}^{1-a}\left(k_{1}-1\right)^{1-a} k_{1} P_{i_{1}, k_{1}, m_{1}} \\
& =\sum_{a=0}^{1}\left(\begin{array}{l}
1 \\
a
\end{array}\right) \mu_{a, a, 1} \sum_{i_{1}=1}^{\infty} \sum_{k_{1}=0}^{\infty} \sum_{m_{1}=0}^{\infty} i_{1}^{1-a}\left(k_{1}-1\right)^{1-a} k_{1} P_{i_{1}, k_{1}, m_{1}} \\
& =\left(\frac{1 !}{0 ! 1 !} \mu_{001} \sum_{i_{1}=1}^{\infty} \sum_{k_{1}=0}^{\infty} \sum_{m_{1}=0}^{\infty} i_{1}\left(k_{1}-1\right) k_{1} P_{i_{1}, k_{1}, m_{1}}\right)+\left(\frac{1 !}{1 ! 0 !} \mu_{111} \sum_{i_{1}=1}^{\infty} \sum_{k_{1}=0}^{\infty} \sum_{m_{1}=0}^{\infty} k_{1} P_{i_{1}, k_{1}, m_{1}}\right) \\
& =\mu_{001} \mu_{120}-\mu_{001} \mu_{110}+\mu_{111} \mu_{010}
\end{aligned}
$$




$$
\begin{aligned}
& \Rightarrow \frac{d \mu_{10100}}{d t}=k_{i} C_{M} C_{R} \\
& -k_{p} C_{M} \sum_{i=1}^{\infty} \sum_{j=0}^{\infty} \sum_{k=0}^{\infty} \sum_{l=0}^{\infty} \sum_{m=0}^{\infty} i k^{2} P_{i j k l m} \\
& +k_{p} C_{M} \sum_{i=1}^{\infty} \sum_{j=0}^{\infty} \sum_{k=0}^{\infty} \sum_{l=0}^{\infty} \sum_{m=0}^{\infty}(i+1) k^{2} P_{i j k l m} \\
& -k_{p} C_{M} \sum_{i=1}^{\infty} \sum_{j=0}^{\infty} \sum_{k=0}^{\infty} \sum_{l=0}^{\infty} \sum_{m=0}^{\infty} i k m P_{i j k l m} \\
& +k_{p} C_{M} \sum_{i=1}^{\infty} \sum_{j=0}^{\infty} \sum_{k=0}^{\infty} \sum_{l=0}^{\infty} \sum_{m=0}^{\infty}(i+1) k m P_{i j k l m} \\
& -k_{t r t} C_{T} \sum_{i=1}^{\infty} \sum_{j=0}^{\infty} \sum_{k=0}^{\infty} \sum_{l=0}^{\infty} \sum_{m=0}^{\infty} i k^{2} P_{i j k l m} \\
& +k_{t r t} C_{T} \sum_{i=1}^{\infty} \sum_{j=0}^{\infty} \sum_{k=0}^{\infty} \sum_{l=0}^{\infty} \sum_{m=0}^{\infty} i(k-1) k P_{i j k l m} \\
& -k_{t r t} \sum_{i=1}^{\infty} \sum_{j=0}^{\infty} \sum_{k=0}^{\infty} \sum_{l=0}^{\infty} \sum_{m=0}^{\infty} i k^{2} P_{i j k l m} \sum_{i_{1}=1}^{\infty} \sum_{j_{1}=0}^{\infty} \sum_{k_{1}=0}^{\infty} \sum_{l_{1}=0}^{\infty} \sum_{m_{1}=0}^{\infty} l_{1} P_{i_{1} j_{1} k_{1} l_{1} m_{1}} \\
& -k_{t r t} \sum_{i=1}^{\infty} \sum_{j=0}^{\infty} \sum_{k=0}^{\infty} \sum_{l=0}^{\infty} \sum_{m=0}^{\infty} i k l P_{i j k l m} \sum_{i_{1}=1}^{\infty} \sum_{j_{1}=0}^{\infty} \sum_{k_{1}=0}^{\infty} \sum_{l_{1}=0}^{\infty} \sum_{m_{1}=0}^{\infty} k_{1} P_{i_{1} j_{1} k_{1} l_{1} m_{1}} \\
& +k_{t r t} \sum_{i=0}^{\infty} \sum_{j=0}^{\infty} \sum_{k=0}^{\infty} \sum_{l=1}^{\infty} \sum_{m=0}^{\infty} i(k-1) k P_{i j k l m} \sum_{i_{1}=1}^{\infty} \sum_{j_{1}=0}^{\infty} \sum_{k_{1}=0}^{\infty} \sum_{l_{1}=0}^{\infty} \sum_{m_{1}=0}^{\infty} l_{1} P_{i_{1} j_{1} k_{1} l_{1} m_{1}} \\
& +k_{t r t} \sum_{i=1}^{\infty} \sum_{j=0}^{\infty} \sum_{k=0}^{\infty} \sum_{l=0}^{\infty} \sum_{m=0}^{\infty} i k l P_{i j k l m} \sum_{i_{1}=1}^{\infty} \sum_{j_{1}=0}^{\infty} \sum_{k_{1}=0}^{\infty} \sum_{l_{1}=0}^{\infty} \sum_{m_{1}=0}^{\infty} k_{1} P_{i_{1} j_{1} k_{1} l_{1} m_{1}} \\
& -k_{\text {trp }} \sum_{i=1}^{\infty} \sum_{j=0}^{\infty} \sum_{k=0}^{\infty} \sum_{l=0}^{\infty} \sum_{m=0}^{\infty} i k^{2} P_{i j k l m} \sum_{i_{1}=1}^{\infty} \sum_{j_{1}=0}^{\infty} \sum_{k_{1}=0}^{\infty} \sum_{l_{1}=0}^{\infty} \sum_{m_{1}=0}^{\infty} i_{1} P_{i_{1} j_{1} k_{1} l_{1} m_{1}} \\
& -k_{t r p} \sum_{i=1}^{\infty} \sum_{j=0}^{\infty} \sum_{k=0}^{\infty} \sum_{l=0}^{\infty} \sum_{m=0}^{\infty} i^{2} k P_{i j k l m} \sum_{i_{1}=1}^{\infty} \sum_{j_{1}=0}^{\infty} \sum_{k_{1}=0}^{\infty} \sum_{l_{1}=0}^{\infty} \sum_{m_{1}=0}^{\infty} k_{1} P_{i_{1} j_{1} k_{1} l_{1} m_{1}} \\
& +k_{t r p} \sum_{i=1}^{\infty} \sum_{j=0}^{\infty} \sum_{k=0}^{\infty} \sum_{l=0}^{\infty} \sum_{m=0}^{\infty} i(k-1) k P_{i j k l m} \sum_{i_{1}=1}^{\infty} \sum_{j_{1}=0}^{\infty} \sum_{k_{1}=0}^{\infty} \sum_{l_{1}=0}^{\infty} \sum_{m_{1}=0}^{\infty} i_{1} P_{i_{1} j_{1} k_{1} l_{1} m_{1}} \\
& +k_{t r p} \sum_{i=1}^{\infty} \sum_{j=0}^{\infty} \sum_{k=0}^{\infty} \sum_{l=0}^{\infty} \sum_{m=0}^{\infty} i^{2}(k+1) P_{i j k l m} \sum_{i_{1}=1}^{\infty} \sum_{j_{1}=0}^{\infty} \sum_{k_{1}=0}^{\infty} \sum_{l_{1}=0}^{\infty} \sum_{m_{1}=0}^{\infty} k_{1} P_{i_{1} j_{1} k_{1} l_{1} m_{1}}
\end{aligned}
$$




$$
\begin{aligned}
& -k_{t r p} \sum_{i=1}^{\infty} \sum_{j=0}^{\infty} \sum_{k=0}^{\infty} \sum_{l=0}^{\infty} \sum_{m=0}^{\infty} i k m P_{i j k l m} \sum_{i_{1}=1}^{\infty} \sum_{j_{1}=0}^{\infty} \sum_{k_{1}=0}^{\infty} \sum_{l_{1}=0}^{\infty} \sum_{m_{1}=0}^{\infty} i_{1} P_{i_{1} j_{1} k_{1} l_{1} m_{1}} \\
& -k_{t r p} \sum_{i=1}^{\infty} \sum_{j=0}^{\infty} \sum_{k=0}^{\infty} \sum_{l=0}^{\infty} \sum_{m=0}^{\infty} i^{2} k P_{i j k l m} \sum_{i_{1}=1}^{\infty} \sum_{j_{1}=0}^{\infty} \sum_{k_{1}=0}^{\infty} \sum_{l_{1}=0}^{\infty} \sum_{m_{1}=0}^{\infty} m_{1} P_{i_{1} j_{1} k_{1} l_{1} m_{1}} \\
& +k_{t r p} \sum_{i=1}^{\infty} \sum_{j=0}^{\infty} \sum_{k=0}^{\infty} \sum_{l=0}^{\infty} \sum_{m=0}^{\infty} i k m P_{i j k l m} \sum_{i_{1}=1}^{\infty} \sum_{j_{1}=0}^{\infty} \sum_{k_{1}=0}^{\infty} \sum_{l_{1}=0}^{\infty} \sum_{m_{1}=0}^{\infty} i_{1} P_{i_{1} j_{1} k_{1} l_{1} m_{1}} \\
& +k_{t r p} \sum_{i=1}^{\infty} \sum_{j=0}^{\infty} \sum_{k=0}^{\infty} \sum_{l=0}^{\infty} \sum_{m=0}^{\infty} i^{2} k P_{i j k l m} \sum_{i_{1}=1}^{\infty} \sum_{j_{1}=0}^{\infty} \sum_{k_{1}=0}^{\infty} \sum_{l_{1}=0}^{\infty} \sum_{m_{1}=0}^{\infty} m_{1} P_{i_{1} j_{1} k_{1} l_{1} m_{1}} \\
& -k_{i n t r} \sum_{i=1}^{\infty} \sum_{j=0}^{\infty} \sum_{k=0}^{\infty} \sum_{l=0}^{\infty} \sum_{m=0}^{\infty} i k^{2} P_{i j k l m} \\
& +k_{\text {intr }} \sum_{i=1}^{\infty} \sum_{j=0}^{\infty} \sum_{k=0}^{\infty} \sum_{l=0}^{\infty} \sum_{m=0}^{\infty} i k^{2} P_{i j k l m} \\
& -k_{r} \sum_{i=1}^{\infty} \sum_{j=0}^{\infty} \sum_{k=0}^{\infty} \sum_{l=0}^{\infty} \sum_{m=0}^{\infty} i k m P_{i j k l m} \\
& +k_{r} \sum_{i=1}^{\infty} \sum_{j=0}^{\infty} \sum_{k=0}^{\infty} \sum_{l=0}^{\infty} \sum_{m=0}^{\infty} i(k+1) m P_{i j k l m} \\
& -k_{t c} \sum_{i=1}^{\infty} \sum_{j=0}^{\infty} \sum_{k=0}^{\infty} \sum_{l=0}^{\infty} \sum_{m=0}^{\infty} i k^{2} P_{i j k l m} \sum_{i_{1}=1}^{\infty} \sum_{j_{1}=0}^{\infty} \sum_{k_{1}=0}^{\infty} \sum_{l_{1}=0}^{\infty} \sum_{m_{1}=0}^{\infty} k_{1} P_{i_{1} j_{1} k_{1} l_{1} m_{1}} \\
& +\frac{1}{2}(2) k_{t c} \sum_{i=1}^{\infty} \sum_{j=0}^{\infty} \sum_{k=0}^{\infty} \sum_{l=0}^{\infty} \sum_{m=0}^{\infty} i k^{2} P_{i j k l m} \sum_{i_{1}=1}^{\infty} \sum_{j_{1}=0}^{\infty} \sum_{k_{1}=0}^{\infty} \sum_{l_{1}=0}^{\infty} \sum_{m_{1}=0}^{\infty} k_{1} P_{i_{1} j_{1} k_{1} l_{1} m_{1}} \\
& -\frac{1}{2}(2) k_{t c} \sum_{i=1}^{\infty} \sum_{j=0}^{\infty} \sum_{k=0}^{\infty} \sum_{l=0}^{\infty} \sum_{m=0}^{\infty} i k P_{i j k l m} \sum_{i_{1}=1}^{\infty} \sum_{j_{1}=0}^{\infty} \sum_{k_{1}=0}^{\infty} \sum_{l_{1}=0}^{\infty} \sum_{m_{1}=0}^{\infty} k_{1} P_{i_{1} j_{1} k_{1} l_{1} m_{1}} \\
& -k_{t c} \sum_{i=1}^{\infty} \sum_{j=0}^{\infty} \sum_{k=0}^{\infty} \sum_{l=0}^{\infty} \sum_{m=0}^{\infty} i k m P_{i j k l m} \sum_{i_{1}=1}^{\infty} \sum_{j_{1}=0}^{\infty} \sum_{k_{1}=0}^{\infty} \sum_{l_{1}=0}^{\infty} \sum_{m_{1}=0}^{\infty} m_{1} P_{i_{1} j_{1} k_{1} l_{1} m_{1}} \\
& +\frac{1}{2}(2) k_{t c} \sum_{i=1}^{\infty} \sum_{j=0}^{\infty} \sum_{k=0}^{\infty} \sum_{l=0}^{\infty} \sum_{m=0}^{\infty} i k m P_{i j k l m} \sum_{i_{1}=1}^{\infty} \sum_{j_{1}=0}^{\infty} \sum_{k_{1}=0}^{\infty} \sum_{l_{1}=0}^{\infty} \sum_{m_{1}=0}^{\infty} m_{1} P_{i_{1} j_{1} k_{1} l_{1} m_{1}} \\
& -k_{t c} \sum_{i=1}^{\infty} \sum_{j=0}^{\infty} \sum_{k=0}^{\infty} \sum_{l=0}^{\infty} \sum_{m=0}^{\infty} i k^{2} P_{i j k l m} \sum_{i_{1}=1}^{\infty} \sum_{j_{1}=0}^{\infty} \sum_{k_{1}=0}^{\infty} \sum_{l_{1}=0}^{\infty} \sum_{m_{1}=0}^{\infty} m_{1} P_{i_{1} j_{1} k_{1} l_{1} m_{1}} \\
& -k_{t c} \sum_{i=1}^{\infty} \sum_{j=0}^{\infty} \sum_{k=0}^{\infty} \sum_{l=0}^{\infty} \sum_{m=0}^{\infty} i k m P_{i j k l m} \sum_{i_{1}=1}^{\infty} \sum_{j_{1}=0}^{\infty} \sum_{k_{1}=0}^{\infty} \sum_{l_{1}=0}^{\infty} \sum_{m_{1}=0}^{\infty} k_{1} P_{i_{1} j_{1} k_{1} l_{1} m_{1}} \\
& +\frac{1}{2}(2) k_{t c} \sum_{i=1}^{\infty} \sum_{j=0}^{\infty} \sum_{k=0}^{\infty} \sum_{l=0}^{\infty} \sum_{m=0}^{\infty} i k^{2} P_{i j k l m} \sum_{i_{1}=1}^{\infty} \sum_{j_{1}=0}^{\infty} \sum_{k_{1}=0}^{\infty} \sum_{l_{1}=0}^{\infty} \sum_{m_{1}=0}^{\infty} m_{1} P_{i_{1} j_{1} k_{1} l_{1} m_{1}} \\
& +\frac{1}{2}(2) k_{t c} \sum_{i=1}^{\infty} \sum_{j=0}^{\infty} \sum_{k=0}^{\infty} \sum_{l=0}^{\infty} \sum_{m=0}^{\infty} i k m P_{i j k l m} \sum_{i_{1}=1}^{\infty} \sum_{j_{1}=0}^{\infty} \sum_{k_{1}=0}^{\infty} \sum_{l_{1}=0}^{\infty} \sum_{m_{1}=0}^{\infty} k_{1} P_{i_{1} j_{1} k_{1} l_{1} m_{1}} \\
& -\frac{1}{2} k_{t c} \sum_{i=1}^{\infty} \sum_{j=0}^{\infty} \sum_{k=0}^{\infty} \sum_{l=0}^{\infty} \sum_{m=0}^{\infty} i k P_{i j k l m} \sum_{i_{1}=1}^{\infty} \sum_{j_{1}=0}^{\infty} \sum_{k_{1}=0}^{\infty} \sum_{l_{1}=0}^{\infty} \sum_{m_{1}=0}^{\infty} m_{1} P_{i_{1} j_{1} k_{1} l_{1} m_{1}} \\
& -\frac{1}{2} k_{t c} \sum_{i=1}^{\infty} \sum_{j=0}^{\infty} \sum_{k=0}^{\infty} \sum_{l=0}^{\infty} \sum_{m=0}^{\infty} i m P_{i j k l m} \sum_{i_{1}=1}^{\infty} \sum_{j_{1}=0}^{\infty} \sum_{k_{1}=0}^{\infty} \sum_{l_{1}=0}^{\infty} \sum_{m_{1}=0}^{\infty} k_{1} P_{i_{1} j_{1} k_{1} l_{1} m_{1}}
\end{aligned}
$$


using Equations (73), (74), (75) and (76).

$$
\begin{aligned}
& \Rightarrow \frac{d \mu_{10100}}{d t}=k_{i} C_{M} C_{R} \\
& +k_{p} C_{M} \sum_{i=1}^{\infty} \sum_{j=0}^{\infty} \sum_{k=0}^{\infty} \sum_{l=0}^{\infty} \sum_{m=0}^{\infty} k^{2} P_{i j k l m} \\
& +k_{p} C_{M} \sum_{i=1}^{\infty} \sum_{j=0}^{\infty} \sum_{k=0}^{\infty} \sum_{l=0}^{\infty} \sum_{m=0}^{\infty} k m P_{i j k l m} \\
& -k_{f i} C_{I} \sum_{i=1}^{\infty} \sum_{j=0}^{\infty} \sum_{k=0}^{\infty} \sum_{l=0}^{\infty} \sum_{m=0}^{\infty} i k P_{i j k l m} \\
& -k_{t r t} C_{T} \sum_{i=1}^{\infty} \sum_{j=0}^{\infty} \sum_{k=0}^{\infty} \sum_{l=0}^{\infty} \sum_{m=0}^{\infty} i k P_{i j k l m} \\
& -k_{\text {trt }} \sum_{i=0}^{\infty} \sum_{j=0}^{\infty} \sum_{k=0}^{\infty} \sum_{l=1}^{\infty} \sum_{m=0}^{\infty} i k P_{i j k l m} \sum_{i_{1}=1}^{\infty} \sum_{j_{1}=0}^{\infty} \sum_{k_{1}=0}^{\infty} \sum_{l_{1}=0}^{\infty} \sum_{m_{1}=0}^{\infty} l_{1} P_{i_{1} j_{1} k_{1} l_{1} m_{1}} \\
& -k_{\text {trp }} \sum_{i=1}^{\infty} \sum_{j=0}^{\infty} \sum_{k=0}^{\infty} \sum_{l=0}^{\infty} \sum_{m=0}^{\infty} i k P_{i j k l m} \sum_{i_{1}=1}^{\infty} \sum_{j_{1}=0}^{\infty} \sum_{k_{1}=0}^{\infty} \sum_{l_{1}=0}^{\infty} \sum_{m_{1}=0}^{\infty} i_{1} P_{i_{1} j_{1} k_{1} l_{1} m_{1}} \\
& +k_{t r p} \sum_{i=1}^{\infty} \sum_{j=0}^{\infty} \sum_{k=0}^{\infty} \sum_{l=0}^{\infty} \sum_{m=0}^{\infty} i^{2} P_{i j k l m} \sum_{i_{1}=1}^{\infty} \sum_{j_{1}=0}^{\infty} \sum_{k_{1}=0}^{\infty} \sum_{l_{1}=0}^{\infty} \sum_{m_{1}=0}^{\infty} k_{1} P_{i_{1} j_{1} k_{1} l_{1} m_{1}} \\
& +k_{r} \sum_{i=1}^{\infty} \sum_{j=0}^{\infty} \sum_{k=0}^{\infty} \sum_{l=0}^{\infty} \sum_{m=0}^{\infty} i m P_{i j k l m} \\
& -k_{t c} \sum_{i=1}^{\infty} \sum_{j=0}^{\infty} \sum_{k=0}^{\infty} \sum_{l=0}^{\infty} \sum_{m=0}^{\infty} i k P_{i j k l m} \sum_{i_{1}=1}^{\infty} \sum_{j_{1}=0}^{\infty} \sum_{k_{1}=0}^{\infty} \sum_{l_{1}=0}^{\infty} \sum_{m_{1}=0}^{\infty} k_{1} P_{i_{1} j_{1} k_{1} l_{1} m_{1}} \\
& -\frac{1}{2} k_{t c} \sum_{i=1}^{\infty} \sum_{j=0}^{\infty} \sum_{k=0}^{\infty} \sum_{l=0}^{\infty} \sum_{m=0}^{\infty} i k P_{i j k l m} \sum_{i_{1}=1}^{\infty} \sum_{j_{1}=0}^{\infty} \sum_{k_{1}=0}^{\infty} \sum_{l_{1}=0}^{\infty} \sum_{m_{1}=0}^{\infty} m_{1} P_{i_{1} j_{1} k_{1} l_{1} m_{1}} \\
& -\frac{1}{2} k_{t c} \sum_{i=1}^{\infty} \sum_{j=0}^{\infty} \sum_{k=0}^{\infty} \sum_{l=0}^{\infty} \sum_{m=0}^{\infty} i m P_{i j k l m} \sum_{i_{1}=1}^{\infty} \sum_{j_{1}=0}^{\infty} \sum_{k_{1}=0}^{\infty} \sum_{l_{1}=0}^{\infty} \sum_{m_{1}=0}^{\infty} k_{1} P_{i_{1} j_{1} k_{1} l_{1} m_{1}} \\
& \Rightarrow \frac{d \mu_{10100}}{d t}=k_{i} C_{M} C_{R}+k_{p} C_{M} \mu_{00200}+k_{p} C_{M} \mu_{00101}-k_{f i} C_{I} \mu_{10100}-k_{t r t} C_{T} \mu_{10100} \\
& -k_{t r t} \mu_{10100} \mu_{00010}-k_{t r p} \mu_{10100} \mu_{10000}+k_{t r p} \mu_{20000} \mu_{00100}+k_{r} \mu_{10001} \\
& -k_{t c} \mu_{10100} \mu_{00100}-\frac{1}{2} k_{t c} \mu_{10100} \mu_{00001}-\frac{1}{2} k_{t c} \mu_{00100} \mu_{10001}
\end{aligned}
$$


3.3.3 Second moment w.r.t. the number of active (uncapped) radicals

$$
\begin{aligned}
& \frac{d \mu_{00200}}{d t}=\sum_{i=1}^{\infty} \sum_{j=0}^{\infty} \sum_{k=0}^{\infty} \sum_{l=0}^{\infty} \sum_{m=0}^{\infty} k^{2} \frac{d P_{i j k l m}}{d t} \\
& =k_{i} C_{M} C_{R} \\
& -k_{p} C_{M} \sum_{i=1}^{\infty} \sum_{j=0}^{\infty} \sum_{k=0}^{\infty} \sum_{l=0}^{\infty} \sum_{m=0}^{\infty} k^{3} P_{i j k l m} \\
& +k_{p} C_{M} \sum_{i=2}^{\infty} \sum_{j=0}^{\infty} \sum_{k=0}^{\infty} \sum_{l=0}^{\infty} \sum_{m=0}^{\infty} k^{3} P_{i-1 j k l m} \\
& -k_{p} C_{M} \sum_{i=1}^{\infty} \sum_{j=0}^{\infty} \sum_{k=0}^{\infty} \sum_{l=0}^{\infty} \sum_{m=0}^{\infty} k^{2} m P_{i j k l m} \\
& +k_{p} C_{M} \sum_{i=2}^{\infty} \sum_{j=0}^{\infty} \sum_{k=0}^{\infty} \sum_{l=0}^{\infty} \sum_{m=0}^{\infty} k^{2} m P_{i-1 j k l m} \\
& -k_{t r t} C_{T} \sum_{i=1}^{\infty} \sum_{j=0}^{\infty} \sum_{k=0}^{\infty} \sum_{l=0}^{\infty} \sum_{m=0}^{\infty} k^{3} P_{i j k l m} \\
& +k_{t r t} C_{T} \sum_{i=1}^{\infty} \sum_{j=0}^{\infty} \sum_{k=0}^{\infty} \sum_{l=1}^{\infty} \sum_{m=0}^{\infty} k^{2}(k+1) P_{i j k+1 l-1 m} \\
& -k_{t r t} \sum_{i=1}^{\infty} \sum_{j=0}^{\infty} \sum_{k=0}^{\infty} \sum_{l=0}^{\infty} \sum_{m=0}^{\infty} k^{3} P_{i j k l m} \sum_{i_{1}=1}^{\infty} \sum_{j_{1}=0}^{\infty} \sum_{k_{1}=0}^{\infty} \sum_{l_{1}=0}^{\infty} \sum_{m_{1}=0}^{\infty} l_{1} P_{i_{1} j_{1} k_{1} l_{1} m_{1}} \\
& -k_{t r t} \sum_{i=1}^{\infty} \sum_{j=0}^{\infty} \sum_{k=0}^{\infty} \sum_{l=0}^{\infty} \sum_{m=0}^{\infty} k^{2} l P_{i j k l m} \sum_{i_{1}=1}^{\infty} \sum_{j_{1}=0}^{\infty} \sum_{k_{1}=0}^{\infty} \sum_{l_{1}=0}^{\infty} \sum_{m_{1}=0}^{\infty} k_{1} P_{i_{1} j_{1} k_{1} l_{1} m_{1}} \\
& +k_{t r t} \sum_{i=1}^{\infty} \sum_{j=0}^{\infty} \sum_{k=0}^{\infty} \sum_{l=1}^{\infty} \sum_{m=0}^{\infty} k^{2}(k+1) P_{i j k+1 l-1 m} \sum_{i_{1}=1}^{\infty} \sum_{j_{1}=0}^{\infty} \sum_{k_{1}=0}^{\infty} \sum_{l_{1}=0}^{\infty} \sum_{m_{1}=0}^{\infty} l_{1} P_{i_{1} j_{1} k_{1} l_{1} m_{1}} \\
& +k_{t r t} \sum_{i=1}^{\infty} \sum_{j=0}^{\infty} \sum_{k=0}^{\infty} \sum_{l=0}^{\infty} \sum_{m=1}^{\infty} k^{2}(l+1) P_{i j k l+1 m-1} \sum_{i_{1}=1}^{\infty} \sum_{j_{1}=0}^{\infty} \sum_{k_{1}=0}^{\infty} \sum_{l_{1}=0}^{\infty} \sum_{m_{1}=0}^{\infty} k_{1} P_{i_{1} j_{1} k_{1} l_{1} m_{1}} \\
& -k_{\operatorname{trp}} \sum_{i=1}^{\infty} \sum_{j=0}^{\infty} \sum_{k=0}^{\infty} \sum_{l=0}^{\infty} \sum_{m=0}^{\infty} k^{3} P_{i j k l m} \sum_{i_{1}=1}^{\infty} \sum_{j_{1}=0}^{\infty} \sum_{k_{1}=0}^{\infty} \sum_{l_{1}=0}^{\infty} \sum_{m_{1}=0}^{\infty} i_{1} P_{i_{1} j_{1} k_{1} l_{1} m_{1}} \\
& -k_{\operatorname{trp}} \sum_{i=1}^{\infty} \sum_{j=0}^{\infty} \sum_{k=0}^{\infty} \sum_{l=0}^{\infty} \sum_{m=0}^{\infty} i k^{2} P_{i j k l m} \sum_{i_{1}=1}^{\infty} \sum_{j_{1}=0}^{\infty} \sum_{k_{1}=0}^{\infty} \sum_{l_{1}=0}^{\infty} \sum_{m_{1}=0}^{\infty} k_{1} P_{i_{1} j_{1} k_{1} l_{1} m_{1}} \\
& +k_{\operatorname{trp}} \sum_{i=1}^{\infty} \sum_{j=0}^{\infty} \sum_{k=0}^{\infty} \sum_{l=0}^{\infty} \sum_{m=0}^{\infty} k^{2}(k+1) P_{i j k+1 l m} \sum_{i_{1}=1}^{\infty} \sum_{j_{1}=0}^{\infty} \sum_{k_{1}=0}^{\infty} \sum_{l_{1}=0}^{\infty} \sum_{m_{1}=0}^{\infty} i_{1} P_{i_{1} j_{1} k_{1} l_{1} m_{1}} \\
& +k_{\operatorname{trp}} \sum_{i=1}^{\infty} \sum_{j=1}^{\infty} \sum_{k=1}^{\infty} \sum_{l=0}^{\infty} \sum_{m=0}^{\infty} i k^{2} P_{i j-1 k-1 l m} \sum_{i_{1}=1}^{\infty} \sum_{j_{1}=0}^{\infty} \sum_{k_{1}=0}^{\infty} \sum_{l_{1}=0}^{\infty} \sum_{m_{1}=0}^{\infty} k_{1} P_{i_{1} j_{1} k_{1} l_{1} m_{1}}
\end{aligned}
$$


$-k_{t r p} \sum_{i=1}^{\infty} \sum_{j=0}^{\infty} \sum_{k=0}^{\infty} \sum_{l=0}^{\infty} \sum_{m=0}^{\infty} k^{2} m P_{i j k l m} \sum_{i_{1}=1}^{\infty} \sum_{j_{1}=0}^{\infty} \sum_{k_{1}=0}^{\infty} \sum_{l_{1}=0}^{\infty} \sum_{m_{1}=0}^{\infty} i_{1} P_{i_{1} j_{1} k_{1} l_{1} m_{1}}$

$-k_{t r p} \sum_{i=1}^{\infty} \sum_{j=0}^{\infty} \sum_{k=0}^{\infty} \sum_{l=0}^{\infty} \sum_{m=0}^{\infty} i k^{2} P_{i j k l m} \sum_{i_{1}=1}^{\infty} \sum_{j_{1}=0}^{\infty} \sum_{k_{1}=0}^{\infty} \sum_{l_{1}=0}^{\infty} \sum_{m_{1}=0}^{\infty} m_{1} P_{i_{1} j_{1} k_{1} l_{1} m_{1}}$

$+k_{t r p} \sum_{i=1}^{\infty} \sum_{j=0}^{\infty} \sum_{k=0}^{\infty} \sum_{l=0}^{\infty} \sum_{m=0}^{\infty} k^{2}(m+1) P_{i j k l m+1} \sum_{i_{1}=1}^{\infty} \sum_{j_{1}=0}^{\infty} \sum_{k_{1}=0}^{\infty} \sum_{l_{1}=0}^{\infty} \sum_{m_{1}=0}^{\infty} i_{1} P_{i_{1} j_{1} k_{1} l_{1} m_{1}}$

$+k_{t r p} \sum_{i=1}^{\infty} \sum_{j=1}^{\infty} \sum_{k=0}^{\infty} \sum_{l=0}^{\infty} \sum_{m=1}^{\infty} i k^{2} P_{i j-1 k l m-1} \sum_{i_{1}=1}^{\infty} \sum_{j_{1}=0}^{\infty} \sum_{k_{1}=0}^{\infty} \sum_{l_{1}=0}^{\infty} \sum_{m_{1}=0}^{\infty} m_{1} P_{i_{1} j_{1} k_{1} l_{1} m_{1}}$

$-k_{i n t r} \sum_{i=1}^{\infty} \sum_{j=0}^{\infty} \sum_{k=0}^{\infty} \sum_{l=0}^{\infty} \sum_{m=0}^{\infty} k^{3} P_{i j k l m}$

$+k_{i n t r} \sum_{i=1}^{\infty} \sum_{j=1}^{\infty} \sum_{k=0}^{\infty} \sum_{l=0}^{\infty} \sum_{m=0}^{\infty} k^{3} P_{i j-1 k l m}$

$-k_{r} \sum_{i=1}^{\infty} \sum_{j=0}^{\infty} \sum_{k=0}^{\infty} \sum_{l=0}^{\infty} \sum_{m=0}^{\infty} k^{2} m P_{i j k l m}$

$+k_{r} \sum_{i=1}^{\infty} \sum_{j=0}^{\infty} \sum_{k=1}^{\infty} \sum_{l=0}^{\infty} \sum_{m=0}^{\infty} k^{2}(m+1) P_{i j k-1 l m+1}$

$-k_{t c} \sum_{i=1}^{\infty} \sum_{j=0}^{\infty} \sum_{k=0}^{\infty} \sum_{l=0}^{\infty} \sum_{m=0}^{\infty} k^{3} P_{i j k l m} \sum_{i_{1}=1}^{\infty} \sum_{j_{1}=0}^{\infty} \sum_{k_{1}=0}^{\infty} \sum_{l_{1}=0}^{\infty} \sum_{m_{1}=0}^{\infty} k_{1} P_{i_{1} j_{1} k_{1} l_{1} m_{1}}$

$+\frac{1}{2} k_{t c} \sum_{i=1}^{\infty} \sum_{j=0}^{\infty} \sum_{k=0}^{\infty} \sum_{l=0}^{\infty} \sum_{m=0}^{\infty} \sum_{i_{1}=1}^{i} \sum_{j_{1}=0}^{j} \sum_{k_{1}=0}^{k+2} \sum_{l_{1}=0}^{l} \sum_{m_{1}=0}^{m} k^{2}\left[k_{1} P_{i_{1} j_{1} k_{1} l_{1} m_{1}}\left(k-k_{1}+2\right) P_{i-i_{1}, j-j_{1}, k-k_{1}+2, l-l_{1} m-m_{1}}\right]$

$-k_{t c} \sum_{i=1}^{\infty} \sum_{j=0}^{\infty} \sum_{k=0}^{\infty} \sum_{l=0}^{\infty} \sum_{m=0}^{\infty} k^{2} m P_{i j k l m} \sum_{i_{1}=1}^{\infty} \sum_{j_{1}=0}^{\infty} \sum_{k_{1}=0}^{\infty} \sum_{l_{1}=0}^{\infty} \sum_{m_{1}=0}^{\infty} m_{1} P_{i_{1} j_{1} k_{1} l_{1} m_{1}}$

$+\frac{1}{2} k_{t c} \sum_{i=1}^{\infty} \sum_{j=0}^{\infty} \sum_{k=0}^{\infty} \sum_{l=0}^{\infty} \sum_{m=0}^{\infty} \sum_{i_{1}=1}^{i} \sum_{j_{1}=0}^{j} \sum_{k_{1}=0}^{k} \sum_{l_{1}=0}^{l} \sum_{m_{1}=0}^{m+2}\left[k^{2} m_{1} P_{i_{1} j_{1} k_{1} l_{1} m_{1}}\left(m+2-m_{1}\right) P_{i-i_{1}, j-j_{1}, k-k_{1}, l-l_{1} m+2-m_{1}}\right]$

$-k_{t c} \sum_{i=1}^{\infty} \sum_{j=0}^{\infty} \sum_{k=0}^{\infty} \sum_{l=0}^{\infty} \sum_{m=0}^{\infty} k^{3} P_{i j k l m} \sum_{i_{1}=1}^{\infty} \sum_{j_{1}=0}^{\infty} \sum_{k_{1}=0}^{\infty} \sum_{l_{1}=0}^{\infty} \sum_{m_{1}=0}^{\infty} m_{1} P_{i_{1} j_{1} k_{1} l_{1} m_{1}}$

$-k_{t c} \sum_{i=1}^{\infty} \sum_{j=0}^{\infty} \sum_{k=0}^{\infty} \sum_{l=0}^{\infty} \sum_{m=0}^{\infty} k^{2} m P_{i j k l m} \sum_{i_{1}=1}^{\infty} \sum_{j_{1}=0}^{\infty} \sum_{k_{1}=0}^{\infty} \sum_{l_{1}=0}^{\infty} \sum_{m_{1}=0}^{\infty} k_{1} P_{i_{1} j_{1} k_{1} l_{1} m_{1}}$

$+\frac{1}{2} k_{t c} \sum_{i=1}^{\infty} \sum_{j=0}^{\infty} \sum_{k=0}^{\infty} \sum_{l=0}^{\infty} \sum_{m=0}^{\infty} \sum_{i_{1}=1}^{i} \sum_{j_{1}=0}^{j} \sum_{k_{1}=0}^{k+1} \sum_{l_{1}=0}^{l} \sum_{m_{1}=0}^{m+1}\left[k^{2} k_{1} P_{i_{1} j_{1} k_{1} l_{1} m_{1}}\left(m+1-m_{1}\right) P_{i-i_{1}, j-j_{1}, k+1-k_{1}, l-l_{1} m+1-m_{1}}\right]$

$+\frac{1}{2} k_{t c} \sum_{i=1}^{\infty} \sum_{j=0}^{\infty} \sum_{k=0}^{\infty} \sum_{l=0}^{\infty} \sum_{m=0}^{\infty} \sum_{i_{1}=1}^{i} \sum_{j_{1}=0}^{j} \sum_{k_{1}=0}^{k+1} \sum_{l_{1}=0}^{l} \sum_{m_{1}=0}^{m+1}\left[k^{2} m_{1} P_{i_{1} j_{1} k_{1} l_{1} m_{1}}\left(k+1-k_{1}\right) P_{i-i_{1}, j-j_{1}, k+1-k_{1}, l-l_{1} m+1-m_{1}}\right]$ 
Now,

$$
\begin{aligned}
\sum_{k=0}^{\infty} \sum_{k_{1}=0}^{k+2} & k^{2} k_{1} P_{k_{1}}\left(k+2-k_{1}\right) P_{k+2-k_{1}} \\
& =\sum_{k=0}^{\infty} \sum_{k_{1}=0}^{k+2}\left[k_{1}+\left(k-k_{1}\right)\right]^{2} k_{1} P_{k_{1}}\left(k+2-k_{1}\right) P_{k+2-k_{1}} \\
& =\sum_{a=0}^{2}\left(\begin{array}{l}
2 \\
a
\end{array}\right) \sum_{k=0}^{\infty} \sum_{k_{1}=0}^{k+2} k_{1}^{1+a} P_{k_{1}}\left(k+2-k_{1}-2\right)^{2-a}\left(k+2-k_{1}\right) P_{k+2-k_{1}} \\
& =\sum_{a=0}^{2}\left(\begin{array}{l}
2 \\
a
\end{array}\right) \sum_{k=0}^{\infty} k^{1+a} P_{k} \sum_{k_{1}=0}^{\infty}\left(k_{1}-2\right)^{2-a} k_{1} P_{k_{1}} \\
& =\sum_{a=0}^{2}\left(\begin{array}{l}
2 \\
a
\end{array}\right) \mu_{1+a} \sum_{k_{1}=0}^{\infty}\left(k_{1}-2\right)^{2-a} k_{1} P_{k_{1}} \\
& =\left(\frac{2 !}{0 ! 2 !} \mu_{1} \sum_{k_{1}=0}^{\infty}\left(k_{1}-2\right)^{2} k_{1} P_{k_{1}}\right)+\left(\frac{2 !}{1 ! 1 !} \mu_{2} \sum_{k_{1}=0}^{\infty}\left(k_{1}-2\right) k_{1} P_{k_{1}}\right)+\left(\frac{2 !}{2 ! 0 !} \mu_{3} \sum_{k_{1}=0}^{\infty} k_{1} P_{k_{1}}\right) \\
= & \mu_{1}\left(\mu_{3}-4 \mu_{2}+4 \mu_{1}\right)+2 \mu_{2}\left(\mu_{2}-2 \mu_{1}\right)+\mu_{3} \mu_{1} \\
= & 2 \mu_{3} \mu_{1}+2 \mu_{2}^{2}-8 \mu_{2} \mu_{1}+4 \mu_{1}^{2}
\end{aligned}
$$

Similarly,

$$
\begin{aligned}
& \sum_{k=0}^{\infty} \sum_{k_{1}=0}^{k+1} \sum_{m=0}^{\infty} \sum_{m_{1}=0}^{m+1} k^{2} k_{1} P_{k_{1}, m_{1}}\left(m+1-m_{1}\right) P_{k+1-k_{1}, m+1-m_{1}} \\
&= \sum_{k=0}^{\infty} \sum_{k_{1}=0}^{k+1} \sum_{m=0}^{\infty} \sum_{m_{1}=0}^{m+1}\left[k_{1}+\left(k-k_{1}\right)\right]^{2} k_{1} P_{k_{1}, m_{1}}\left(m+1-m_{1}\right) P_{k+1-k_{1}, m+1-m_{1}} \\
&= \sum_{a=0}^{2}\left(\begin{array}{l}
2 \\
a
\end{array}\right) \sum_{k=0}^{\infty} \sum_{k_{1}=0}^{k+1} \sum_{m=0}^{\infty} \sum_{m_{1}=0}^{m+1} k_{1}^{1+a} P_{k_{1}, m_{1}}\left(k+1-k_{1}-1\right)^{2-a}\left(m+1-m_{1}\right) P_{k+1-k_{1}, m+1-m_{1}} \\
&= \sum_{a=0}^{2}\left(\begin{array}{l}
2 \\
a
\end{array}\right) \sum_{k=0}^{\infty} \sum_{m=0}^{\infty} k^{1+a} P_{k, m} \sum_{k_{1}=0}^{\infty} \sum_{m_{1}=0}^{\infty}\left(k_{1}-1\right)^{2-a} m_{1} P_{k_{1}, m_{1}} \\
&= \sum_{a=0}^{2}\left(\begin{array}{l}
2 \\
a
\end{array}\right) \mu_{1+a, 0} \sum_{k_{1}=0}^{\infty} \sum_{m_{1}=0}^{\infty}\left(k_{1}-1\right)^{2-a} m_{1} P_{k_{1}, m_{1}} \\
&=\left(\frac{2 !}{0 ! 2 !} \mu_{10} \sum_{k_{1}=0}^{\infty} \sum_{m_{1}=0}^{\infty}\left(k_{1}-1\right)^{2} m_{1} P_{k_{1}, m_{1}}\right)+\left(\frac{2 !}{1 ! 1 !} \mu_{20} \sum_{k_{1}=0}^{\infty} \sum_{m_{1}=0}^{\infty}\left(k_{1}-1\right) m_{1} P_{k_{1}, m_{1}}\right) \\
& \quad+\left(\frac{2 !}{2 ! 0 !} \mu_{30} \sum_{k_{1}=0}^{\infty} \sum_{m_{1}=0}^{\infty} m_{1} P_{k_{1}, m_{1}}\right) \\
&=\mu_{10}\left(\mu_{21}-2 \mu_{11}+\mu_{01}\right)+2 \mu_{20}\left(\mu_{11}-\mu_{01}\right)+\mu_{30} \mu_{01} \\
&=\mu_{30} \mu_{01}+\mu_{21} \mu_{10}+2 \mu_{20} \mu_{11}-2 \mu_{20} \mu_{01}-2 \mu_{11} \mu_{10}+\mu_{10} \mu_{01}
\end{aligned}
$$


and

$$
\begin{aligned}
\sum_{k=0}^{\infty} \sum_{k_{1}=0}^{k+1} & \sum_{m=0}^{\infty} \sum_{m_{1}=0}^{m+1} k^{2} m_{1} P_{k_{1}, m_{1}}\left(k+1-k_{1}\right) P_{k+1-k_{1}, m+1-m_{1}} \\
= & \sum_{k=0}^{\infty} \sum_{k_{1}=0}^{k+1} \sum_{m=0}^{\infty} \sum_{m_{1}=0}^{m+1}\left[k_{1}+\left(k-k_{1}\right)\right]^{2} m_{1} P_{k_{1}, m_{1}}\left(k+1-k_{1}\right) P_{k+1-k_{1}, m+1-m_{1}} \\
= & \sum_{a=0}^{2}\left(\begin{array}{l}
2 \\
a
\end{array}\right) \sum_{k=0}^{\infty} \sum_{k_{1}=0}^{k+1} \sum_{m=0}^{\infty} \sum_{m_{1}=0}^{m+1} k_{1}^{a} m_{1} P_{k_{1}, m_{1}}\left(k+1-k_{1}-1\right)^{2-a}\left(k+1-k_{1}\right) P_{k+1-k_{1}, m_{+1}-m_{1}} \\
= & \sum_{a=0}^{2}\left(\begin{array}{l}
2 \\
a
\end{array}\right) \sum_{k=0}^{\infty} \sum_{m=0}^{\infty} k^{a} m P_{k, m} \sum_{k_{1}=0}^{\infty} \sum_{m_{1}=0}^{\infty}\left(k_{1}-1\right)^{2-a} k_{1} P_{k_{1}, m_{1}} \\
= & \sum_{a=0}^{2}\left(\begin{array}{l}
2 \\
a
\end{array}\right) \mu_{a, 1} \sum_{k_{1}=0}^{\infty} \sum_{m_{1}=0}^{\infty}\left(k_{1}-1\right)^{2-a} k_{1} P_{k_{1}, m_{1}} \\
= & \left(\frac{2 !}{0 ! 2 !} \mu_{01} \sum_{k_{1}=0}^{\infty} \sum_{m_{1}=0}^{\infty}\left(k_{1}-1\right)^{2} k_{1} P_{k_{1}, m_{1}}\right)+\left(\frac{2 !}{1 ! 1 !} \mu_{11} \sum_{k_{1}=0}^{\infty} \sum_{m_{1}=0}^{\infty}\left(k_{1}-1\right) k_{1} P_{k_{1}, m_{1}}\right) \\
& \quad+\left(\frac{2 !}{2 ! 0 !} \mu_{21} \sum_{k_{1}=0}^{\infty} \sum_{m_{1}=0}^{\infty} k_{1} P_{k_{1}, m_{1}}\right) \\
= & \mu_{01}\left(\mu_{30}-2 \mu_{20}+\mu_{10}\right)+2 \mu_{11}\left(\mu_{20}-\mu_{10}\right)+\mu_{21} \mu_{10} \\
= & \mu_{30} \mu_{01}+\mu_{21} \mu_{10}+2 \mu_{20} \mu_{11}-2 \mu_{20} \mu_{01}-2 \mu_{11} \mu_{10}+\mu_{10} \mu_{01}
\end{aligned}
$$

$$
\begin{aligned}
& \Rightarrow \frac{d \mu_{00200}}{d t}=k_{i} C_{M} C_{R} \\
& \quad-k_{p} C_{M} \sum_{i=1}^{\infty} \sum_{j=0}^{\infty} \sum_{k=0}^{\infty} \sum_{l=0}^{\infty} \sum_{m=0}^{\infty} k^{3} P_{i j k l m} \\
& +k_{p} C_{M} \sum_{i=1}^{\infty} \sum_{j=0}^{\infty} \sum_{k=0}^{\infty} \sum_{l=0}^{\infty} \sum_{m=0}^{\infty} k^{3} P_{i j k l m} \\
& \quad-k_{p} C_{M} \sum_{i=1}^{\infty} \sum_{j=0}^{\infty} \sum_{k=0}^{\infty} \sum_{l=0}^{\infty} \sum_{m=0}^{\infty} k^{2} m P_{i j k l m} \\
& +k_{p} C_{M} \sum_{i=1}^{\infty} \sum_{j=0}^{\infty} \sum_{k=0}^{\infty} \sum_{l=0}^{\infty} \sum_{m=0}^{\infty} k^{2} m P_{i j k l m} \\
& \quad-k_{t r t} C_{T} \sum_{i=1}^{\infty} \sum_{j=0}^{\infty} \sum_{k=0}^{\infty} \sum_{l=0}^{\infty} \sum_{m=0}^{\infty} k^{3} P_{i j k l m} \\
& +k_{t r t} C_{T} \sum_{i=1}^{\infty} \sum_{j=0}^{\infty} \sum_{k=0}^{\infty} \sum_{l=0}^{\infty} \sum_{m=0}^{\infty}(k-1)^{2} k P_{i j k l m}
\end{aligned}
$$




$$
\begin{aligned}
& -k_{t r t} \sum_{i=1}^{\infty} \sum_{j=0}^{\infty} \sum_{k=0}^{\infty} \sum_{l=0}^{\infty} \sum_{m=0}^{\infty} k^{3} P_{i j k l m} \sum_{i_{1}=1}^{\infty} \sum_{j_{1}=0}^{\infty} \sum_{k_{1}=0}^{\infty} \sum_{l_{1}=0}^{\infty} \sum_{m_{1}=0}^{\infty} l_{1} P_{i_{1} j_{1} k_{1} l_{1} m_{1}} \\
& -k_{t r t} \sum_{i=1}^{\infty} \sum_{j=0}^{\infty} \sum_{k=0}^{\infty} \sum_{l=0}^{\infty} \sum_{m=0}^{\infty} k^{2} l P_{i j k l m} \sum_{i_{1}=1}^{\infty} \sum_{j_{1}=0}^{\infty} \sum_{k_{1}=0}^{\infty} \sum_{l_{1}=0}^{\infty} \sum_{m_{1}=0}^{\infty} k_{1} P_{i_{1} j_{1} k_{1} l_{1} m_{1}} \\
& +k_{\text {trt }} \sum_{i=1}^{\infty} \sum_{j=0}^{\infty} \sum_{k=0}^{\infty} \sum_{l=0}^{\infty} \sum_{m=0}^{\infty}(k-1)^{2} k P_{i j k l m} \sum_{i_{1}=1}^{\infty} \sum_{j_{1}=0}^{\infty} \sum_{k_{1}=0}^{\infty} \sum_{l_{1}=0}^{\infty} \sum_{m_{1}=0}^{\infty} l_{1} P_{i_{1} j_{1} k_{1} l_{1} m_{1}} \\
& +k_{\text {trt }} \sum_{i=1}^{\infty} \sum_{j=0}^{\infty} \sum_{k=0}^{\infty} \sum_{l=0}^{\infty} \sum_{m=0}^{\infty} k^{2} l P_{i j k l m} \sum_{i_{1}=1}^{\infty} \sum_{j_{1}=0}^{\infty} \sum_{k_{1}=0}^{\infty} \sum_{l_{1}=0}^{\infty} \sum_{m_{1}=0}^{\infty} k_{1} P_{i_{1} j_{1} k_{1} l_{1} m_{1}} \\
& -k_{\operatorname{trp}} \sum_{i=1}^{\infty} \sum_{j=0}^{\infty} \sum_{k=0}^{\infty} \sum_{l=0}^{\infty} \sum_{m=0}^{\infty} k^{3} P_{i j k l m} \sum_{i_{1}=1}^{\infty} \sum_{j_{1}=0}^{\infty} \sum_{k_{1}=0}^{\infty} \sum_{l_{1}=0}^{\infty} \sum_{m_{1}=0}^{\infty} i_{1} P_{i_{1} j_{1} k_{1} l_{1} m_{1}} \\
& -k_{\operatorname{trp}} \sum_{i=1}^{\infty} \sum_{j=0}^{\infty} \sum_{k=0}^{\infty} \sum_{l=0}^{\infty} \sum_{m=0}^{\infty} i k^{2} P_{i j k l m} \sum_{i_{1}=1}^{\infty} \sum_{j_{1}=0}^{\infty} \sum_{k_{1}=0}^{\infty} \sum_{l_{1}=0}^{\infty} \sum_{m_{1}=0}^{\infty} k_{1} P_{i_{1} j_{1} k_{1} l_{1} m_{1}} \\
& +k_{\text {trp }} \sum_{i=1}^{\infty} \sum_{j=0}^{\infty} \sum_{k=0}^{\infty} \sum_{l=0}^{\infty} \sum_{m=0}^{\infty}(k-1)^{2} k P_{i j k l m} \sum_{i_{1}=1}^{\infty} \sum_{j_{1}=0}^{\infty} \sum_{k_{1}=0}^{\infty} \sum_{l_{1}=0}^{\infty} \sum_{m_{1}=0}^{\infty} i_{1} P_{i_{1} j_{1} k_{1} l_{1} m_{1}} \\
& +k_{\operatorname{trp}} \sum_{i=1}^{\infty} \sum_{j=0}^{\infty} \sum_{k=0}^{\infty} \sum_{l=0}^{\infty} \sum_{m=0}^{\infty} i(k+1)^{2} P_{i j k l m} \sum_{i_{1}=1}^{\infty} \sum_{j_{1}=0}^{\infty} \sum_{k_{1}=0}^{\infty} \sum_{l_{1}=0}^{\infty} \sum_{m_{1}=0}^{\infty} k_{1} P_{i_{1} j_{1} k_{1} l_{1} m_{1}} \\
& -k_{\operatorname{trp}} \sum_{i=1}^{\infty} \sum_{j=0}^{\infty} \sum_{k=0}^{\infty} \sum_{l=0}^{\infty} \sum_{m=0}^{\infty} k^{2} m P_{i j k l m} \sum_{i_{1}=1}^{\infty} \sum_{j_{1}=0}^{\infty} \sum_{k_{1}=0}^{\infty} \sum_{l_{1}=0}^{\infty} \sum_{m_{1}=0}^{\infty} i_{1} P_{i_{1} j_{1} k_{1} l_{1} m_{1}} \\
& -k_{\operatorname{trp}} \sum_{i=1}^{\infty} \sum_{j=0}^{\infty} \sum_{k=0}^{\infty} \sum_{l=0}^{\infty} \sum_{m=0}^{\infty} i k^{2} P_{i j k l m} \sum_{i_{1}=1}^{\infty} \sum_{j_{1}=0}^{\infty} \sum_{k_{1}=0}^{\infty} \sum_{l_{1}=0}^{\infty} \sum_{m_{1}=0}^{\infty} m_{1} P_{i_{1} j_{1} k_{1} l_{1} m_{1}} \\
& +k_{t r p} \sum_{i=1}^{\infty} \sum_{j=0}^{\infty} \sum_{k=0}^{\infty} \sum_{l=0}^{\infty} \sum_{m=0}^{\infty} k^{2} m P_{i j k l m} \sum_{i_{1}=1}^{\infty} \sum_{j_{1}=0}^{\infty} \sum_{k_{1}=0}^{\infty} \sum_{l_{1}=0}^{\infty} \sum_{m_{1}=0}^{\infty} i_{1} P_{i_{1} j_{1} k_{1} l_{1} m_{1}} \\
& +k_{\text {trp }} \sum_{i=1}^{\infty} \sum_{j=0}^{\infty} \sum_{k=0}^{\infty} \sum_{l=0}^{\infty} \sum_{m=0}^{\infty} i k^{2} P_{i j k l m} \sum_{i_{1}=1}^{\infty} \sum_{j_{1}=0}^{\infty} \sum_{k_{1}=0}^{\infty} \sum_{l_{1}=0}^{\infty} \sum_{m_{1}=0}^{\infty} m_{1} P_{i_{1} j_{1} k_{1} l_{1} m_{1}} \\
& -k_{\text {intr }} \sum_{i=1}^{\infty} \sum_{j=0}^{\infty} \sum_{k=0}^{\infty} \sum_{l=0}^{\infty} \sum_{m=0}^{\infty} k^{3} P_{i j k l m} \\
& +k_{i n t r} \sum_{i=1}^{\infty} \sum_{j=0}^{\infty} \sum_{k=0}^{\infty} \sum_{l=0}^{\infty} \sum_{m=0}^{\infty} k^{3} P_{i j k l m} \\
& -k_{r} \sum_{i=1}^{\infty} \sum_{j=0}^{\infty} \sum_{k=0}^{\infty} \sum_{l=0}^{\infty} \sum_{m=0}^{\infty} k^{2} m P_{i j k l m} \\
& +k_{r} \sum_{i=1}^{\infty} \sum_{j=0}^{\infty} \sum_{k=0}^{\infty} \sum_{l=0}^{\infty} \sum_{m=0}^{\infty}(k+1)^{2} m P_{i j k l m}
\end{aligned}
$$




$$
\begin{aligned}
& -k_{t c} \sum_{i=1}^{\infty} \sum_{j=0}^{\infty} \sum_{k=0}^{\infty} \sum_{l=0}^{\infty} \sum_{m=0}^{\infty} k^{3} P_{i j k l m} \sum_{i_{1}=1}^{\infty} \sum_{j_{1}=0}^{\infty} \sum_{k_{1}=0}^{\infty} \sum_{l_{1}=0}^{\infty} \sum_{m_{1}=0}^{\infty} k_{1} P_{i_{1} j_{1} k_{1} l_{1} m_{1}} \\
& +\frac{1}{2}(2) k_{t c} \sum_{i=1}^{\infty} \sum_{j=0}^{\infty} \sum_{k=0}^{\infty} \sum_{l=0}^{\infty} \sum_{m=0}^{\infty} k^{3} P_{i j k l m} \sum_{i_{1}=1}^{\infty} \sum_{j_{1}=0}^{\infty} \sum_{k_{1}=0}^{\infty} \sum_{l_{1}=0}^{\infty} \sum_{m_{1}=0}^{\infty} k_{1} P_{i_{1} j_{1} k_{1} l_{1} m_{1}} \\
& +\frac{1}{2}(2) k_{t c} \sum_{i=1}^{\infty} \sum_{j=0}^{\infty} \sum_{k=0}^{\infty} \sum_{l=0}^{\infty} \sum_{m=0}^{\infty} k^{2} P_{i j k l m} \sum_{i_{1}=1}^{\infty} \sum_{j_{1}=0}^{\infty} \sum_{k_{1}=0}^{\infty} \sum_{l_{1}=0}^{\infty} \sum_{m_{1}=0}^{\infty} k_{1}^{2} P_{i_{1} j_{1} k_{1} l_{1} m_{1}} \\
& -\frac{1}{2}(8) k_{t c} \sum_{i=1}^{\infty} \sum_{j=0}^{\infty} \sum_{k=0}^{\infty} \sum_{l=0}^{\infty} \sum_{m=0}^{\infty} k P_{i j k l m} \sum_{i_{1}=1}^{\infty} \sum_{j_{1}=0}^{\infty} \sum_{k_{1}=0}^{\infty} \sum_{l_{1}=0}^{\infty} \sum_{m_{1}=0}^{\infty} k_{1}^{2} P_{i_{1} j_{1} k_{1} l_{1} m_{1}} \\
& +\frac{1}{2}(4) k_{t c} \sum_{i=1}^{\infty} \sum_{j=0}^{\infty} \sum_{k=0}^{\infty} \sum_{l=0}^{\infty} \sum_{m=0}^{\infty} k P_{i j k l m} \sum_{i_{1}=1}^{\infty} \sum_{j_{1}=0}^{\infty} \sum_{k_{1}=0}^{\infty} \sum_{l_{1}=0}^{\infty} \sum_{m_{1}=0}^{\infty} k_{1} P_{i_{1} j_{1} k_{1} l_{1} m_{1}} \\
& -k_{t c} \sum_{i=1}^{\infty} \sum_{j=0}^{\infty} \sum_{k=0}^{\infty} \sum_{l=0}^{\infty} \sum_{m=0}^{\infty} k^{2} m P_{i j k l m} \sum_{i_{1}=1}^{\infty} \sum_{j_{1}=0}^{\infty} \sum_{k_{1}=0}^{\infty} \sum_{l_{1}=0}^{\infty} \sum_{m_{1}=0}^{\infty} m_{1} P_{i_{1} j_{1} k_{1} l_{1} m_{1}} \\
& +\frac{1}{2}(2) k_{t c} \sum_{i=1}^{\infty} \sum_{j=0}^{\infty} \sum_{k=0}^{\infty} \sum_{l=0}^{\infty} \sum_{m=0}^{\infty} k^{2} m P_{i j k l m} \sum_{i_{1}=1}^{\infty} \sum_{j_{1}=0}^{\infty} \sum_{k_{1}=0}^{\infty} \sum_{l_{1}=0}^{\infty} \sum_{m_{1}=0}^{\infty} m_{1} P_{i_{1} j_{1} k_{1} l_{1} m_{1}} \\
& -k_{t c} \sum_{i=1}^{\infty} \sum_{j=0}^{\infty} \sum_{k=0}^{\infty} \sum_{l=0}^{\infty} \sum_{m=0}^{\infty} k^{3} P_{i j k l m} \sum_{i_{1}=1}^{\infty} \sum_{j_{1}=0}^{\infty} \sum_{k_{1}=0}^{\infty} \sum_{l_{1}=0}^{\infty} \sum_{m_{1}=0}^{\infty} m_{1} P_{i_{1} j_{1} k_{1} l_{1} m_{1}} \\
& -k_{t c} \sum_{i=1}^{\infty} \sum_{j=0}^{\infty} \sum_{k=0}^{\infty} \sum_{l=0}^{\infty} \sum_{m=0}^{\infty} k^{2} m P_{i j k l m} \sum_{i_{1}=1}^{\infty} \sum_{j_{1}=0}^{\infty} \sum_{k_{1}=0}^{\infty} \sum_{l_{1}=0}^{\infty} \sum_{m_{1}=0}^{\infty} k_{1} P_{i_{1} j_{1} k_{1} l_{1} m_{1}} \\
& +\frac{1}{2}(2) k_{t c} \sum_{i=1}^{\infty} \sum_{j=0}^{\infty} \sum_{k=0}^{\infty} \sum_{l=0}^{\infty} \sum_{m=0}^{\infty} k^{3} P_{i j k l m} \sum_{i_{1}=1}^{\infty} \sum_{j_{1}=0}^{\infty} \sum_{k_{1}=0}^{\infty} \sum_{l_{1}=0}^{\infty} \sum_{m_{1}=0}^{\infty} m_{1} P_{i_{1} j_{1} k_{1} l_{1} m_{1}} \\
& +\frac{1}{2}(2) k_{t c} \sum_{i=1}^{\infty} \sum_{j=0}^{\infty} \sum_{k=0}^{\infty} \sum_{l=0}^{\infty} \sum_{m=0}^{\infty} k^{2} m P_{i j k l m} \sum_{i_{1}=1}^{\infty} \sum_{j_{1}=0}^{\infty} \sum_{k_{1}=0}^{\infty} \sum_{l_{1}=0}^{\infty} \sum_{m_{1}=0}^{\infty} k_{1} P_{i_{1} j_{1} k_{1} l_{1} m_{1}} \\
& +\frac{1}{2}(4) k_{t c} \sum_{i=1}^{\infty} \sum_{j=0}^{\infty} \sum_{k=0}^{\infty} \sum_{l=0}^{\infty} \sum_{m=0}^{\infty} k^{2} P_{i j k l m} \sum_{i_{1}=1}^{\infty} \sum_{j_{1}=0}^{\infty} \sum_{k_{1}=0}^{\infty} \sum_{l_{1}=0}^{\infty} \sum_{m_{1}=0}^{\infty} k_{1} m_{1} P_{i_{1} j_{1} k_{1} l_{1} m_{1}} \\
& -\frac{1}{2}(4) k_{t c} \sum_{i=1}^{\infty} \sum_{j=0}^{\infty} \sum_{k=0}^{\infty} \sum_{l=0}^{\infty} \sum_{m=0}^{\infty} k^{2} P_{i j k l m} \sum_{i_{1}=1}^{\infty} \sum_{j_{1}=0}^{\infty} \sum_{k_{1}=0}^{\infty} \sum_{l_{1}=0}^{\infty} \sum_{m_{1}=0}^{\infty} m_{1} P_{i_{1} j_{1} k_{1} l_{1} m_{1}} \\
& -\frac{1}{2}(4) k_{t c} \sum_{i=1}^{\infty} \sum_{j=0}^{\infty} \sum_{k=0}^{\infty} \sum_{l=0}^{\infty} \sum_{m=0}^{\infty} k m P_{i j k l m} \sum_{i_{1}=1}^{\infty} \sum_{j_{1}=0}^{\infty} \sum_{k_{1}=0}^{\infty} \sum_{l_{1}=0}^{\infty} \sum_{m_{1}=0}^{\infty} k_{1} P_{i_{1} j_{1} k_{1} l_{1} m_{1}} \\
& +\frac{1}{2}(2) k_{t c} \sum_{i=1}^{\infty} \sum_{j=0}^{\infty} \sum_{k=0}^{\infty} \sum_{l=0}^{\infty} \sum_{m=0}^{\infty} k P_{i j k l m} \sum_{i_{1}=1}^{\infty} \sum_{j_{1}=0}^{\infty} \sum_{k_{1}=0}^{\infty} \sum_{l_{1}=0}^{\infty} \sum_{m_{1}=0}^{\infty} m_{1} P_{i_{1} j_{1} k_{1} l_{1} m_{1}}
\end{aligned}
$$

using Equations (67), (81), (82) and (83). 


$$
\begin{aligned}
& \Rightarrow \frac{d \mu_{00200}}{d t}=k_{i} C_{M} C_{R} \\
& +k_{t r t} C_{T} \sum_{i=1}^{\infty} \sum_{j=0}^{\infty} \sum_{k=0}^{\infty} \sum_{l=0}^{\infty} \sum_{m=0}^{\infty}\left(k-2 k^{2}\right) P_{i j k l m} \\
& +k_{t r t} \sum_{i=1}^{\infty} \sum_{j=0}^{\infty} \sum_{k=0}^{\infty} \sum_{l=0}^{\infty} \sum_{m=0}^{\infty}\left(k-2 k^{2}\right) P_{i j k l m} \sum_{i_{1}=1}^{\infty} \sum_{j_{1}=0}^{\infty} \sum_{k_{1}=0}^{\infty} \sum_{l_{1}=0}^{\infty} \sum_{m_{1}=0}^{\infty} l_{1} P_{i_{1} j_{1} k_{1} l_{1} m_{1}} \\
& +k_{\text {trp }} \sum_{i=1}^{\infty} \sum_{j=0}^{\infty} \sum_{k=0}^{\infty} \sum_{l=0}^{\infty} \sum_{m=0}^{\infty}\left(k-2 k^{2}\right) P_{i j k l m} \sum_{i_{1}=1}^{\infty} \sum_{j_{1}=0}^{\infty} \sum_{k_{1}=0}^{\infty} \sum_{l_{1}=0}^{\infty} \sum_{m_{1}=0}^{\infty} i_{1} P_{i_{1} j_{1} k_{1} l_{1} m_{1}} \\
& +k_{t r p} \sum_{i=1}^{\infty} \sum_{j=0}^{\infty} \sum_{k=0}^{\infty} \sum_{l=0}^{\infty} \sum_{m=0}^{\infty}(2 i k+i) P_{i j k l m} \sum_{i_{1}=1}^{\infty} \sum_{j_{1}=0}^{\infty} \sum_{k_{1}=0}^{\infty} \sum_{l_{1}=0}^{\infty} \sum_{m_{1}=0}^{\infty} k_{1} P_{i_{1} j_{1} k_{1} l_{1} m_{1}} \\
& +k_{r} \sum_{i=1}^{\infty} \sum_{j=0}^{\infty} \sum_{k=0}^{\infty} \sum_{l=0}^{\infty} \sum_{m=0}^{\infty}(2 k m+m) P_{i j k l m} \\
& +\frac{1}{2}(2) k_{t c} \sum_{i=1}^{\infty} \sum_{j=0}^{\infty} \sum_{k=0}^{\infty} \sum_{l=0}^{\infty} \sum_{m=0}^{\infty} k^{2} P_{i j k l m} \sum_{i_{1}=1}^{\infty} \sum_{j_{1}=0}^{\infty} \sum_{k_{1}=0}^{\infty} \sum_{l_{1}=0}^{\infty} \sum_{m_{1}=0}^{\infty} k_{1}^{2} P_{i_{1} j_{1} k_{1} l_{1} m_{1}} \\
& -\frac{1}{2}(8) k_{t c} \sum_{i=1}^{\infty} \sum_{j=0}^{\infty} \sum_{k=0}^{\infty} \sum_{l=0}^{\infty} \sum_{m=0}^{\infty} k P_{i j k l m} \sum_{i_{1}=1}^{\infty} \sum_{j_{1}=0}^{\infty} \sum_{k_{1}=0}^{\infty} \sum_{l_{1}=0}^{\infty} \sum_{m_{1}=0}^{\infty} k_{1}^{2} P_{i_{1} j_{1} k_{1} l_{1} m_{1}} \\
& +\frac{1}{2}(4) k_{t c} \sum_{i=1}^{\infty} \sum_{j=0}^{\infty} \sum_{k=0}^{\infty} \sum_{l=0}^{\infty} \sum_{m=0}^{\infty} k P_{i j k l m} \sum_{i_{1}=1}^{\infty} \sum_{j_{1}=0}^{\infty} \sum_{k_{1}=0}^{\infty} \sum_{l_{1}=0}^{\infty} \sum_{m_{1}=0}^{\infty} k_{1} P_{i_{1} j_{1} k_{1} l_{1} m_{1}} \\
& +\frac{1}{2}(4) k_{t c} \sum_{i=1}^{\infty} \sum_{j=0}^{\infty} \sum_{k=0}^{\infty} \sum_{l=0}^{\infty} \sum_{m=0}^{\infty} k^{2} P_{i j k l m} \sum_{i_{1}=1}^{\infty} \sum_{j_{1}=0}^{\infty} \sum_{k_{1}=0}^{\infty} \sum_{l_{1}=0}^{\infty} \sum_{m_{1}=0}^{\infty} k_{1} m_{1} P_{i_{1} j_{1} k_{1} l_{1} m_{1}} \\
& -\frac{1}{2}(4) k_{t c} \sum_{i=1}^{\infty} \sum_{j=0}^{\infty} \sum_{k=0}^{\infty} \sum_{l=0}^{\infty} \sum_{m=0}^{\infty} k^{2} P_{i j k l m} \sum_{i_{1}=1}^{\infty} \sum_{j_{1}=0}^{\infty} \sum_{k_{1}=0}^{\infty} \sum_{l_{1}=0}^{\infty} \sum_{m_{1}=0}^{\infty} m_{1} P_{i_{1} j_{1} k_{1} l_{1} m_{1}} \\
& -\frac{1}{2}(4) k_{t c} \sum_{i=1}^{\infty} \sum_{j=0}^{\infty} \sum_{k=0}^{\infty} \sum_{l=0}^{\infty} \sum_{m=0}^{\infty} k m P_{i j k l m} \sum_{i_{1}=1}^{\infty} \sum_{j_{1}=0}^{\infty} \sum_{k_{1}=0}^{\infty} \sum_{l_{1}=0}^{\infty} \sum_{m_{1}=0}^{\infty} k_{1} P_{i_{1} j_{1} k_{1} l_{1} m_{1}} \\
& +\frac{1}{2}(2) k_{t c} \sum_{i=1}^{\infty} \sum_{j=0}^{\infty} \sum_{k=0}^{\infty} \sum_{l=0}^{\infty} \sum_{m=0}^{\infty} k P_{i j k l m} \sum_{i_{1}=1}^{\infty} \sum_{j_{1}=0}^{\infty} \sum_{k_{1}=0}^{\infty} \sum_{l_{1}=0}^{\infty} \sum_{m_{1}=0}^{\infty} m_{1} P_{i_{1} j_{1} k_{1} l_{1} m_{1}} \\
& \Rightarrow \frac{d \mu_{00200}}{d t}=k_{i} C_{M} C_{R}+k_{t r t} C_{T}\left(\mu_{00100}-2 \mu_{00200}\right) \\
& +k_{t r t} \mu_{00010}\left(\mu_{00100}-2 \mu_{00200}\right)+k_{t r p} \mu_{10000}\left(\mu_{00100}-2 \mu_{00200}\right)+k_{t r p} \mu_{00100}\left(2 \mu_{10100}+\mu_{10000}\right) \\
& +k_{r}\left(2 \mu_{00101}+\mu_{00001}\right)+k_{t c}\left[\left(\mu_{00200}\right)^{2}-4 \mu_{00200} \mu_{00100}+2\left(\mu_{00100}\right)^{2}\right] \\
& +2 k_{t c} \mu_{00200}\left(\mu_{00101}-\mu_{00001}\right)+k_{t c} \mu_{00100}\left(\mu_{00001}-2 \mu_{00101}\right)
\end{aligned}
$$


3.3.4 Second moment w.r.t. the number of repeat units and unrelaxed (just uncapped) radicals

$$
\begin{aligned}
& \frac{d \mu_{10001}}{d t}=\sum_{i=1}^{\infty} \sum_{j=0}^{\infty} \sum_{k=0}^{\infty} \sum_{l=0}^{\infty} \sum_{m=0}^{\infty} i m \frac{d P_{i j k l m}}{d t} \\
& =-k_{p} C_{M} \sum_{i=1}^{\infty} \sum_{j=0}^{\infty} \sum_{k=0}^{\infty} \sum_{l=0}^{\infty} \sum_{m=0}^{\infty} i k m P_{i j k l m} \\
& +k_{p} C_{M} \sum_{i=2}^{\infty} \sum_{j=0}^{\infty} \sum_{k=0}^{\infty} \sum_{l=0}^{\infty} \sum_{m=0}^{\infty} i k m P_{i-1 j k l m} \\
& -k_{p} C_{M} \sum_{i=1}^{\infty} \sum_{j=0}^{\infty} \sum_{k=0}^{\infty} \sum_{l=0}^{\infty} \sum_{m=0}^{\infty} i m^{2} P_{i j k l m} \\
& +k_{p} C_{M} \sum_{i=2}^{\infty} \sum_{j=0}^{\infty} \sum_{k=0}^{\infty} \sum_{l=0}^{\infty} \sum_{m=0}^{\infty} i m^{2} P_{i-1 j k l m} \\
& -k_{t r t} C_{T} \sum_{i=1}^{\infty} \sum_{j=0}^{\infty} \sum_{k=0}^{\infty} \sum_{l=0}^{\infty} \sum_{m=0}^{\infty} i k m P_{i j k l m} \\
& +k_{t r t} C_{T} \sum_{i=1}^{\infty} \sum_{j=0}^{\infty} \sum_{k=0}^{\infty} \sum_{l=1}^{\infty} \sum_{m=0}^{\infty} i(k+1) m P_{i j k+1 l-1 m} \\
& -k_{\text {trt }} \sum_{i=1}^{\infty} \sum_{j=0}^{\infty} \sum_{k=0}^{\infty} \sum_{l=0}^{\infty} \sum_{m=0}^{\infty} i k m P_{i j k l m} \sum_{i_{1}=1}^{\infty} \sum_{j_{1}=0}^{\infty} \sum_{k_{1}=0}^{\infty} \sum_{l_{1}=0}^{\infty} \sum_{m_{1}=0}^{\infty} l_{1} P_{i_{1} j_{1} k_{1} l_{1} m_{1}} \\
& -k_{\text {trt }} \sum_{i=1}^{\infty} \sum_{j=0}^{\infty} \sum_{k=0}^{\infty} \sum_{l=0}^{\infty} \sum_{m=0}^{\infty} i \operatorname{lm} P_{i j k l m} \sum_{i_{1}=1}^{\infty} \sum_{j_{1}=0}^{\infty} \sum_{k_{1}=0}^{\infty} \sum_{l_{1}=0}^{\infty} \sum_{m_{1}=0}^{\infty} k_{1} P_{i_{1} j_{1} k_{1} l_{1} m_{1}} \\
& +k_{t r t} \sum_{i=1}^{\infty} \sum_{j=0}^{\infty} \sum_{k=0}^{\infty} \sum_{l=1}^{\infty} \sum_{m=0}^{\infty} i(k+1) m P_{i j k+1 l-1 m} \sum_{i_{1}=1}^{\infty} \sum_{j_{1}=0}^{\infty} \sum_{k_{1}=0}^{\infty} \sum_{l_{1}=0}^{\infty} \sum_{m_{1}=0}^{\infty} l_{1} P_{i_{1} j_{1} k_{1} l_{1} m_{1}} \\
& +k_{t r t} \sum_{i=1}^{\infty} \sum_{j=0}^{\infty} \sum_{k=0}^{\infty} \sum_{l=0}^{\infty} \sum_{m=1}^{\infty} i(l+1) m P_{i j k l+1 m-1} \sum_{i_{1}=1}^{\infty} \sum_{j_{1}=0}^{\infty} \sum_{k_{1}=0}^{\infty} \sum_{l_{1}=0}^{\infty} \sum_{m_{1}=0}^{\infty} k_{1} P_{i_{1} j_{1} k_{1} l_{1} m_{1}} \\
& -k_{t r p} \sum_{i=1}^{\infty} \sum_{j=0}^{\infty} \sum_{k=0}^{\infty} \sum_{l=0}^{\infty} \sum_{m=0}^{\infty} i k m P_{i j k l m} \sum_{i_{1}=1}^{\infty} \sum_{j_{1}=0}^{\infty} \sum_{k_{1}=0}^{\infty} \sum_{l_{1}=0}^{\infty} \sum_{m_{1}=0}^{\infty} i_{1} P_{i_{1} j_{1} k_{1} l_{1} m_{1}} \\
& -k_{\operatorname{trp}} \sum_{i=1}^{\infty} \sum_{j=0}^{\infty} \sum_{k=0}^{\infty} \sum_{l=0}^{\infty} \sum_{m=0}^{\infty} i^{2} m P_{i j k l m} \sum_{i_{1}=1}^{\infty} \sum_{j_{1}=0}^{\infty} \sum_{k_{1}=0}^{\infty} \sum_{l_{1}=0}^{\infty} \sum_{m_{1}=0}^{\infty} k_{1} P_{i_{1} j_{1} k_{1} l_{1} m_{1}} \\
& +k_{t r p} \sum_{i=1}^{\infty} \sum_{j=0}^{\infty} \sum_{k=0}^{\infty} \sum_{l=0}^{\infty} \sum_{m=0}^{\infty} i(k+1) m P_{i j k+1 l m} \sum_{i_{1}=1}^{\infty} \sum_{j_{1}=0}^{\infty} \sum_{k_{1}=0}^{\infty} \sum_{l_{1}=0}^{\infty} \sum_{m_{1}=0}^{\infty} i_{1} P_{i_{1} j_{1} k_{1} l_{1} m_{1}} \\
& +k_{t r p} \sum_{i=1}^{\infty} \sum_{j=1}^{\infty} \sum_{k=1}^{\infty} \sum_{l=0}^{\infty} \sum_{m=0}^{\infty} i^{2} m P_{i j-1 k-1 l m} \sum_{i_{1}=1}^{\infty} \sum_{j_{1}=0}^{\infty} \sum_{k_{1}=0}^{\infty} \sum_{l_{1}=0}^{\infty} \sum_{m_{1}=0}^{\infty} k_{1} P_{i_{1} j_{1} k_{1} l_{1} m_{1}}
\end{aligned}
$$


$-k_{t r p} \sum_{i=1}^{\infty} \sum_{j=0}^{\infty} \sum_{k=0}^{\infty} \sum_{l=0}^{\infty} \sum_{m=0}^{\infty} i m^{2} P_{i j k l m} \sum_{i_{1}=1}^{\infty} \sum_{j_{1}=0}^{\infty} \sum_{k_{1}=0}^{\infty} \sum_{l_{1}=0}^{\infty} \sum_{m_{1}=0}^{\infty} i_{1} P_{i_{1} j_{1} k_{1} l_{1} m_{1}}$

$-k_{t r p} \sum_{i=1}^{\infty} \sum_{j=0}^{\infty} \sum_{k=0}^{\infty} \sum_{l=0}^{\infty} \sum_{m=0}^{\infty} i^{2} m P_{i j k l m} \sum_{i_{1}=1}^{\infty} \sum_{j_{1}=0}^{\infty} \sum_{k_{1}=0}^{\infty} \sum_{l_{1}=0}^{\infty} \sum_{m_{1}=0}^{\infty} m_{1} P_{i_{1} j_{1} k_{1} l_{1} m_{1}}$

$+k_{t r p} \sum_{i=1}^{\infty} \sum_{j=0}^{\infty} \sum_{k=0}^{\infty} \sum_{l=0}^{\infty} \sum_{m=0}^{\infty} i m(m+1) P_{i j k l m+1} \sum_{i_{1}=1}^{\infty} \sum_{j_{1}=0}^{\infty} \sum_{k_{1}=0}^{\infty} \sum_{l_{1}=0}^{\infty} \sum_{m_{1}=0}^{\infty} i_{1} P_{i_{1} j_{1} k_{1} l_{1} m_{1}}$

$+k_{\text {trp }} \sum_{i=1}^{\infty} \sum_{j=1}^{\infty} \sum_{k=0}^{\infty} \sum_{l=0}^{\infty} \sum_{m=1}^{\infty} i^{2} m P_{i j-1 k l m-1} \sum_{i_{1}=1}^{\infty} \sum_{j_{1}=0}^{\infty} \sum_{k_{1}=0}^{\infty} \sum_{l_{1}=0}^{\infty} \sum_{m_{1}=0}^{\infty} m_{1} P_{i_{1} j_{1} k_{1} l_{1} m_{1}}$

$-k_{\text {intr }} \sum_{i=1}^{\infty} \sum_{j=0}^{\infty} \sum_{k=0}^{\infty} \sum_{l=0}^{\infty} \sum_{m=0}^{\infty} i k m P_{i j k l m}$

$+k_{i n t r} \sum_{i=1}^{\infty} \sum_{j=1}^{\infty} \sum_{k=0}^{\infty} \sum_{l=0}^{\infty} \sum_{m=0}^{\infty} i k m P_{i j-1 k l m}$

$-k_{r} \sum_{i=1}^{\infty} \sum_{j=0}^{\infty} \sum_{k=0}^{\infty} \sum_{l=0}^{\infty} \sum_{m=0}^{\infty} i m^{2} P_{i j k l m}$

$+k_{r} \sum_{i=1}^{\infty} \sum_{j=0}^{\infty} \sum_{k=1}^{\infty} \sum_{l=0}^{\infty} \sum_{m=0}^{\infty} i m(m+1) P_{i j k-1 l m+1}$

$-k_{t c} \sum_{i=1}^{\infty} \sum_{j=0}^{\infty} \sum_{k=0}^{\infty} \sum_{l=0}^{\infty} \sum_{m=0}^{\infty} i k m P_{i j k l m} \sum_{i_{1}=1}^{\infty} \sum_{j_{1}=0}^{\infty} \sum_{k_{1}=0}^{\infty} \sum_{l_{1}=0}^{\infty} \sum_{m_{1}=0}^{\infty} k_{1} P_{i_{1} j_{1} k_{1} l_{1} m_{1}}$

$+\frac{1}{2} k_{t c} \sum_{i=1}^{\infty} \sum_{j=0}^{\infty} \sum_{k=0}^{\infty} \sum_{l=0}^{\infty} \sum_{m=0}^{\infty} \sum_{i_{1}=1}^{i} \sum_{j_{1}=0}^{j} \sum_{k_{1}=0}^{k+2} \sum_{l_{1}=0}^{l} \sum_{m_{1}=0}^{m}\left[i m k_{1} P_{i_{1} j_{1} k_{1} l_{1} m_{1}}\left(k+2-k_{1}\right) P_{i-i_{1}, j-j_{1}, k+2-k_{1}, l-l_{1} m-m_{1}}\right]$

$-k_{t c} \sum_{i=1}^{\infty} \sum_{j=0}^{\infty} \sum_{k=0}^{\infty} \sum_{l=0}^{\infty} \sum_{m=0}^{\infty} i m^{2} P_{i j k l m} \sum_{i_{1}=1}^{\infty} \sum_{j_{1}=0}^{\infty} \sum_{k_{1}=0}^{\infty} \sum_{l_{1}=0}^{\infty} \sum_{m_{1}=0}^{\infty} m_{1} P_{i_{1} j_{1} k_{1} l_{1} m_{1}}$

$+\frac{1}{2} k_{t c} \sum_{i=1}^{\infty} \sum_{j=0}^{\infty} \sum_{k=0}^{\infty} \sum_{l=0}^{\infty} \sum_{m=0}^{\infty} \sum_{i_{1}=1}^{i} \sum_{j_{1}=0}^{j} \sum_{k_{1}=0}^{k} \sum_{l_{1}=0}^{l} \sum_{m_{1}=0}^{m+2}\left[i m m_{1} P_{i_{1} j_{1} k_{1} l_{1} m_{1}}\left(m+2-m_{1}\right) P_{i-i_{1}, j-j_{1}, k-k_{1}, l-l_{1} m+2-m_{1}}\right]$

$-k_{t c} \sum_{i=1}^{\infty} \sum_{j=0}^{\infty} \sum_{k=0}^{\infty} \sum_{l=0}^{\infty} \sum_{m=0}^{\infty} i k m P_{i j k l m} \sum_{i_{1}=1}^{\infty} \sum_{j_{1}=0}^{\infty} \sum_{k_{1}=0}^{\infty} \sum_{l_{1}=0}^{\infty} \sum_{m_{1}=0}^{\infty} m_{1} P_{i_{1} j_{1} k_{1} l_{1} m_{1}}$

$-k_{t c} \sum_{i=1}^{\infty} \sum_{j=0}^{\infty} \sum_{k=0}^{\infty} \sum_{l=0}^{\infty} \sum_{m=0}^{\infty} i m^{2} P_{i j k l m} \sum_{i_{1}=1}^{\infty} \sum_{j_{1}=0}^{\infty} \sum_{k_{1}=0}^{\infty} \sum_{l_{1}=0}^{\infty} \sum_{m_{1}=0}^{\infty} k_{1} P_{i_{1} j_{1} k_{1} l_{1} m_{1}}$

$+\frac{1}{2} k_{t c} \sum_{i=1}^{\infty} \sum_{j=0}^{\infty} \sum_{k=0}^{\infty} \sum_{l=0}^{\infty} \sum_{m=0}^{\infty} \sum_{i_{1}=1}^{i} \sum_{j_{1}=0}^{j} \sum_{k_{1}=0}^{k+1} \sum_{l_{1}=0}^{l} \sum_{m_{1}=0}^{m+1}\left[i m k_{1} P_{i_{1} j_{1} k_{1} l_{1} m_{1}}\left(m+1-m_{1}\right) P_{i-i_{1}, j-j_{1}, k+1-k_{1}, l-l_{1} m+1-m_{1}}\right]$

$+\frac{1}{2} k_{t c} \sum_{i=1}^{\infty} \sum_{j=0}^{\infty} \sum_{k=0}^{\infty} \sum_{l=0}^{\infty} \sum_{m=0}^{\infty} \sum_{i_{1}=1}^{i} \sum_{j_{1}=0}^{j} \sum_{k_{1}=0}^{k+1} \sum_{l_{1}=0}^{l} \sum_{m_{1}=0}^{m+1}\left[i m m_{1} P_{i_{1} j_{1} k_{1} l_{1} m_{1}}\left(k+1-k_{1}\right) P_{i-i_{1}, j-j_{1}, k+1-k_{1}, l-l_{1} m+1-m_{1}}\right]$ 


$$
\begin{aligned}
& \Rightarrow \frac{d \mu_{10001}}{d t}=-k_{p} C_{M} \sum_{i=1}^{\infty} \sum_{j=0}^{\infty} \sum_{k=0}^{\infty} \sum_{l=0}^{\infty} \sum_{m=0}^{\infty} i k m P_{i j k l m} \\
& +k_{p} C_{M} \sum_{i=1}^{\infty} \sum_{j=0}^{\infty} \sum_{k=0}^{\infty} \sum_{l=0}^{\infty} \sum_{m=0}^{\infty}(i+1) k m P_{i j k l m} \\
& -k_{p} C_{M} \sum_{i=1}^{\infty} \sum_{j=0}^{\infty} \sum_{k=0}^{\infty} \sum_{l=0}^{\infty} \sum_{m=0}^{\infty} i m^{2} P_{i j k l m} \\
& +k_{p} C_{M} \sum_{i=1}^{\infty} \sum_{j=0}^{\infty} \sum_{k=0}^{\infty} \sum_{l=0}^{\infty} \sum_{m=0}^{\infty}(i+1) m^{2} P_{i j k l m} \\
& -k_{t r t} C_{T} \sum_{i=1}^{\infty} \sum_{j=0}^{\infty} \sum_{k=0}^{\infty} \sum_{l=0}^{\infty} \sum_{m=0}^{\infty} i k m P_{i j k l m} \\
& +k_{t r t} C_{T} \sum_{i=1}^{\infty} \sum_{j=0}^{\infty} \sum_{k=0}^{\infty} \sum_{l=0}^{\infty} \sum_{m=0}^{\infty} i k m P_{i j k l m} \\
& -k_{t r t} \sum_{i=1}^{\infty} \sum_{j=0}^{\infty} \sum_{k=0}^{\infty} \sum_{l=0}^{\infty} \sum_{m=0}^{\infty} i k m P_{i j k l m} \sum_{i_{1}=1}^{\infty} \sum_{j_{1}=0}^{\infty} \sum_{k_{1}=0}^{\infty} \sum_{l_{1}=0}^{\infty} \sum_{m_{1}=0}^{\infty} l_{1} P_{i_{1} j_{1} k_{1} l_{1} m_{1}} \\
& -k_{t r t} \sum_{i=1}^{\infty} \sum_{j=0}^{\infty} \sum_{k=0}^{\infty} \sum_{l=0}^{\infty} \sum_{m=0}^{\infty} i l m P_{i j k l m} \sum_{i_{1}=1}^{\infty} \sum_{j_{1}=0}^{\infty} \sum_{k_{1}=0}^{\infty} \sum_{l_{1}=0}^{\infty} \sum_{m_{1}=0}^{\infty} k_{1} P_{i_{1} j_{1} k_{1} l_{1} m_{1}} \\
& +k_{t r t} \sum_{i=1}^{\infty} \sum_{j=0}^{\infty} \sum_{k=0}^{\infty} \sum_{l=0}^{\infty} \sum_{m=0}^{\infty} i k m P_{i j k l m} \sum_{i_{1}=1}^{\infty} \sum_{j_{1}=0}^{\infty} \sum_{k_{1}=0}^{\infty} \sum_{l_{1}=0}^{\infty} \sum_{m_{1}=0}^{\infty} l_{1} P_{i_{1} j_{1} k_{1} l_{1} m_{1}} \\
& +k_{t r t} \sum_{i=1}^{\infty} \sum_{j=0}^{\infty} \sum_{k=0}^{\infty} \sum_{l=0}^{\infty} \sum_{m=0}^{\infty} i l(m+1) P_{i j k l m} \sum_{i_{1}=1}^{\infty} \sum_{j_{1}=0}^{\infty} \sum_{k_{1}=0}^{\infty} \sum_{l_{1}=0}^{\infty} \sum_{m_{1}=0}^{\infty} k_{1} P_{i_{1} j_{1} k_{1} l_{1} m_{1}} \\
& -k_{\text {trp }} \sum_{i=1}^{\infty} \sum_{j=0}^{\infty} \sum_{k=0}^{\infty} \sum_{l=0}^{\infty} \sum_{m=0}^{\infty} i k m P_{i j k l m} \sum_{i_{1}=1}^{\infty} \sum_{j_{1}=0}^{\infty} \sum_{k_{1}=0}^{\infty} \sum_{l_{1}=0}^{\infty} \sum_{m_{1}=0}^{\infty} i_{1} P_{i_{1} j_{1} k_{1} l_{1} m_{1}} \\
& -k_{\text {trp }} \sum_{i=1}^{\infty} \sum_{j=0}^{\infty} \sum_{k=0}^{\infty} \sum_{l=0}^{\infty} \sum_{m=0}^{\infty} i^{2} m P_{i j k l m} \sum_{i_{1}=1}^{\infty} \sum_{j_{1}=0}^{\infty} \sum_{k_{1}=0}^{\infty} \sum_{l_{1}=0}^{\infty} \sum_{m_{1}=0}^{\infty} k_{1} P_{i_{1} j_{1} k_{1} l_{1} m_{1}} \\
& +k_{t r p} \sum_{i=1}^{\infty} \sum_{j=0}^{\infty} \sum_{k=0}^{\infty} \sum_{l=0}^{\infty} \sum_{m=0}^{\infty} i k m P_{i j k l m} \sum_{i_{1}=1}^{\infty} \sum_{j_{1}=0}^{\infty} \sum_{k_{1}=0}^{\infty} \sum_{l_{1}=0}^{\infty} \sum_{m_{1}=0}^{\infty} i_{1} P_{i_{1} j_{1} k_{1} l_{1} m_{1}} \\
& +k_{\text {trp }} \sum_{i=1}^{\infty} \sum_{j=1}^{\infty} \sum_{k=1}^{\infty} \sum_{l=0}^{\infty} \sum_{m=0}^{\infty} i^{2} m P_{i j k l m} \sum_{i_{1}=1}^{\infty} \sum_{j_{1}=0}^{\infty} \sum_{k_{1}=0}^{\infty} \sum_{l_{1}=0}^{\infty} \sum_{m_{1}=0}^{\infty} k_{1} P_{i_{1} j_{1} k_{1} l_{1} m_{1}}
\end{aligned}
$$




$$
\begin{aligned}
& -k_{t r p} \sum_{i=1}^{\infty} \sum_{j=0}^{\infty} \sum_{k=0}^{\infty} \sum_{l=0}^{\infty} \sum_{m=0}^{\infty} i m^{2} P_{i j k l m} \sum_{i_{1}=1}^{\infty} \sum_{j_{1}=0}^{\infty} \sum_{k_{1}=0}^{\infty} \sum_{l_{1}=0}^{\infty} \sum_{m_{1}=0}^{\infty} i_{1} P_{i_{1} j_{1} k_{1} l_{1} m_{1}} \\
& -k_{t r p} \sum_{i=1}^{\infty} \sum_{j=0}^{\infty} \sum_{k=0}^{\infty} \sum_{l=0}^{\infty} \sum_{m=0}^{\infty} i^{2} m P_{i j k l m} \sum_{i_{1}=1}^{\infty} \sum_{j_{1}=0}^{\infty} \sum_{k_{1}=0}^{\infty} \sum_{l_{1}=0}^{\infty} \sum_{m_{1}=0}^{\infty} m_{1} P_{i_{1} j_{1} k_{1} l_{1} m_{1}} \\
& +k_{\text {trp }} \sum_{i=1}^{\infty} \sum_{j=0}^{\infty} \sum_{k=0}^{\infty} \sum_{l=0}^{\infty} \sum_{m=0}^{\infty} i(m-1) m P_{i j k l m} \sum_{i_{1}=1}^{\infty} \sum_{j_{1}=0}^{\infty} \sum_{k_{1}=0}^{\infty} \sum_{l_{1}=0}^{\infty} \sum_{m_{1}=0}^{\infty} i_{1} P_{i_{1} j_{1} k_{1} l_{1} m_{1}} \\
& +k_{\text {trp }} \sum_{i=1}^{\infty} \sum_{j=0}^{\infty} \sum_{k=0}^{\infty} \sum_{l=0}^{\infty} \sum_{m=0}^{\infty} i^{2}(m+1) P_{i j k l m} \sum_{i_{1}=1}^{\infty} \sum_{j_{1}=0}^{\infty} \sum_{k_{1}=0}^{\infty} \sum_{l_{1}=0}^{\infty} \sum_{m_{1}=0}^{\infty} m_{1} P_{i_{1} j_{1} k_{1} l_{1} m_{1}} \\
& -k_{i n t r} \sum_{i=1}^{\infty} \sum_{j=0}^{\infty} \sum_{k=0}^{\infty} \sum_{l=0}^{\infty} \sum_{m=0}^{\infty} i k m P_{i j k l m} \\
& +k_{i n t r} \sum_{i=1}^{\infty} \sum_{j=0}^{\infty} \sum_{k=0}^{\infty} \sum_{l=0}^{\infty} \sum_{m=0}^{\infty} i k m P_{i j k l m} \\
& -k_{r} \sum_{i=1}^{\infty} \sum_{j=0}^{\infty} \sum_{k=0}^{\infty} \sum_{l=0}^{\infty} \sum_{m=0}^{\infty} i m^{2} P_{i j k l m} \\
& +k_{r} \sum_{i=1}^{\infty} \sum_{j=0}^{\infty} \sum_{k=0}^{\infty} \sum_{l=0}^{\infty} \sum_{m=0}^{\infty} i(m-1) m P_{i j k l m} \\
& -k_{t c} \sum_{i=1}^{\infty} \sum_{j=0}^{\infty} \sum_{k=0}^{\infty} \sum_{l=0}^{\infty} \sum_{m=0}^{\infty} i k m P_{i j k l m} \sum_{i_{1}=1}^{\infty} \sum_{j_{1}=0}^{\infty} \sum_{k_{1}=0}^{\infty} \sum_{l_{1}=0}^{\infty} \sum_{m_{1}=0}^{\infty} k_{1} P_{i_{1} j_{1} k_{1} l_{1} m_{1}} \\
& +\frac{1}{2}(2) k_{t c} \sum_{i=1}^{\infty} \sum_{j=0}^{\infty} \sum_{k=0}^{\infty} \sum_{l=0}^{\infty} \sum_{m=0}^{\infty} i k m P_{i j k l m} \sum_{i_{1}=1}^{\infty} \sum_{j_{1}=0}^{\infty} \sum_{k_{1}=0}^{\infty} \sum_{l_{1}=0}^{\infty} \sum_{m_{1}=0}^{\infty} k_{1} P_{i_{1} j_{1} k_{1} l_{1} m_{1}} \\
& -k_{t c} \sum_{i=1}^{\infty} \sum_{j=0}^{\infty} \sum_{k=0}^{\infty} \sum_{l=0}^{\infty} \sum_{m=0}^{\infty} i m^{2} P_{i j k l m} \sum_{i_{1}=1}^{\infty} \sum_{j_{1}=0}^{\infty} \sum_{k_{1}=0}^{\infty} \sum_{l_{1}=0}^{\infty} \sum_{m_{1}=0}^{\infty} m_{1} P_{i_{1} j_{1} k_{1} l_{1} m_{1}} \\
& +\frac{1}{2}(2) k_{t c} \sum_{i=1}^{\infty} \sum_{j=0}^{\infty} \sum_{k=0}^{\infty} \sum_{l=0}^{\infty} \sum_{m=0}^{\infty} i m^{2} P_{i j k l m} \sum_{i_{1}=1}^{\infty} \sum_{j_{1}=0}^{\infty} \sum_{k_{1}=0}^{\infty} \sum_{l_{1}=0}^{\infty} \sum_{m_{1}=0}^{\infty} m_{1} P_{i_{1} j_{1} k_{1} l_{1} m_{1}} \\
& -\frac{1}{2}(2) k_{t c} \sum_{i=1}^{\infty} \sum_{j=0}^{\infty} \sum_{k=0}^{\infty} \sum_{l=0}^{\infty} \sum_{m=0}^{\infty} i m P_{i j k l m} \sum_{i_{1}=1}^{\infty} \sum_{j_{1}=0}^{\infty} \sum_{k_{1}=0}^{\infty} \sum_{l_{1}=0}^{\infty} \sum_{m_{1}=0}^{\infty} m_{1} P_{i_{1} j_{1} k_{1} l_{1} m_{1}} \\
& -k_{t c} \sum_{i=1}^{\infty} \sum_{j=0}^{\infty} \sum_{k=0}^{\infty} \sum_{l=0}^{\infty} \sum_{m=0}^{\infty} i k m P_{i j k l m} \sum_{i_{1}=1}^{\infty} \sum_{j_{1}=0}^{\infty} \sum_{k_{1}=0}^{\infty} \sum_{l_{1}=0}^{\infty} \sum_{m_{1}=0}^{\infty} m_{1} P_{i_{1} j_{1} k_{1} l_{1} m_{1}} \\
& -k_{t c} \sum_{i=1}^{\infty} \sum_{j=0}^{\infty} \sum_{k=0}^{\infty} \sum_{l=0}^{\infty} \sum_{m=0}^{\infty} i m^{2} P_{i j k l m} \sum_{i_{1}=1}^{\infty} \sum_{j_{1}=0}^{\infty} \sum_{k_{1}=0}^{\infty} \sum_{l_{1}=0}^{\infty} \sum_{m_{1}=0}^{\infty} k_{1} P_{i_{1} j_{1} k_{1} l_{1} m_{1}}
\end{aligned}
$$




$$
\begin{aligned}
& +\frac{1}{2}(2) k_{t c} \sum_{i=1}^{\infty} \sum_{j=0}^{\infty} \sum_{k=0}^{\infty} \sum_{l=0}^{\infty} \sum_{m=0}^{\infty} i k m P_{i j k l m} \sum_{i_{1}=1}^{\infty} \sum_{j_{1}=0}^{\infty} \sum_{k_{1}=0}^{\infty} \sum_{l_{1}=0}^{\infty} \sum_{m_{1}=0}^{\infty} m_{1} P_{i_{1} j_{1} k_{1} l_{1} m_{1}} \\
& +\frac{1}{2}(2) k_{t c} \sum_{i=1}^{\infty} \sum_{j=0}^{\infty} \sum_{k=0}^{\infty} \sum_{l=0}^{\infty} \sum_{m=0}^{\infty} i m^{2} P_{i j k l m} \sum_{i_{1}=1}^{\infty} \sum_{j_{1}=0}^{\infty} \sum_{k_{1}=0}^{\infty} \sum_{l_{1}=0}^{\infty} \sum_{m_{1}=0}^{\infty} k_{1} P_{i_{1} j_{1} k_{1} l_{1} m_{1}} \\
& -\frac{1}{2} k_{t c} \sum_{i=1}^{\infty} \sum_{j=0}^{\infty} \sum_{k=0}^{\infty} \sum_{l=0}^{\infty} \sum_{m=0}^{\infty} i k P_{i j k l m} \sum_{i_{1}=1}^{\infty} \sum_{j_{1}=0}^{\infty} \sum_{k_{1}=0}^{\infty} \sum_{l_{1}=0}^{\infty} \sum_{m_{1}=0}^{\infty} m_{1} P_{i_{1} j_{1} k_{1} l_{1} m_{1}} \\
& -\frac{1}{2} k_{t c} \sum_{i=1}^{\infty} \sum_{j=0}^{\infty} \sum_{k=0}^{\infty} \sum_{l=0}^{\infty} \sum_{m=0}^{\infty} i m P_{i j k l m} \sum_{i_{1}=1}^{\infty} \sum_{j_{1}=0}^{\infty} \sum_{k_{1}=0}^{\infty} \sum_{l_{1}=0}^{\infty} \sum_{m_{1}=0}^{\infty} k_{1} P_{i_{1} j_{1} k_{1} l_{1} m_{1}}
\end{aligned}
$$

using Equation (73), (74), (75) and (76).

$$
\begin{aligned}
& \Rightarrow \frac{d \mu_{10001}}{d t}=k_{p} C_{M} \sum_{i=1}^{\infty} \sum_{j=0}^{\infty} \sum_{k=0}^{\infty} \sum_{l=0}^{\infty} \sum_{m=0}^{\infty} k m P_{i j k l m} \\
& +k_{p} C_{M} \sum_{i=1}^{\infty} \sum_{j=0}^{\infty} \sum_{k=0}^{\infty} \sum_{l=0}^{\infty} \sum_{m=0}^{\infty} m^{2} P_{i j k l m} \\
& +k_{t r t} \sum_{i=1}^{\infty} \sum_{j=0}^{\infty} \sum_{k=0}^{\infty} \sum_{l=0}^{\infty} \sum_{m=0}^{\infty} i l P_{i j k l m} \sum_{i_{1}=1}^{\infty} \sum_{j_{1}=0}^{\infty} \sum_{k_{1}=0}^{\infty} \sum_{l_{1}=0}^{\infty} \sum_{m_{1}=0}^{\infty} k_{1} P_{i_{1} j_{1} k_{1} l_{1} m_{1}} \\
& -k_{\text {trp }} \sum_{i=1}^{\infty} \sum_{j=0}^{\infty} \sum_{k=0}^{\infty} \sum_{l=0}^{\infty} \sum_{m=0}^{\infty} i m P_{i j k l m} \sum_{i_{1}=1}^{\infty} \sum_{j_{1}=0}^{\infty} \sum_{k_{1}=0}^{\infty} \sum_{l_{1}=0}^{\infty} \sum_{m_{1}=0}^{\infty} i_{1} P_{i_{1} j_{1} k_{1} l_{1} m_{1}} \\
& +k_{t r p} \sum_{i=1}^{\infty} \sum_{j=0}^{\infty} \sum_{k=0}^{\infty} \sum_{l=0}^{\infty} \sum_{m=0}^{\infty} i^{2} P_{i j k l m} \sum_{i_{1}=1}^{\infty} \sum_{j_{1}=0}^{\infty} \sum_{k_{1}=0}^{\infty} \sum_{l_{1}=0}^{\infty} \sum_{m_{1}=0}^{\infty} m_{1} P_{i_{1} j_{1} k_{1} l_{1} m_{1}} \\
& -k_{r} \sum_{i=1}^{\infty} \sum_{j=0}^{\infty} \sum_{k=0}^{\infty} \sum_{l=0}^{\infty} \sum_{m=0}^{\infty} i m P_{i j k l m} \\
& -\frac{1}{2}(2) k_{t c} \sum_{i=1}^{\infty} \sum_{j=0}^{\infty} \sum_{k=0}^{\infty} \sum_{l=0}^{\infty} \sum_{m=0}^{\infty} i m P_{i j k l m} \sum_{i_{1}=1}^{\infty} \sum_{j_{1}=0}^{\infty} \sum_{k_{1}=0}^{\infty} \sum_{l_{1}=0}^{\infty} \sum_{m_{1}=0}^{\infty} m_{1} P_{i_{1} j_{1} k_{1} l_{1} m_{1}} \\
& -\frac{1}{2} k_{t c} \sum_{i=1}^{\infty} \sum_{j=0}^{\infty} \sum_{k=0}^{\infty} \sum_{l=0}^{\infty} \sum_{m=0}^{\infty} i k P_{i j k l m} \sum_{i_{1}=1}^{\infty} \sum_{j_{1}=0}^{\infty} \sum_{k_{1}=0}^{\infty} \sum_{l_{1}=0}^{\infty} \sum_{m_{1}=0}^{\infty} m_{1} P_{i_{1} j_{1} k_{1} l_{1} m_{1}} \\
& -\frac{1}{2} k_{t c} \sum_{i=1}^{\infty} \sum_{j=0}^{\infty} \sum_{k=0}^{\infty} \sum_{l=0}^{\infty} \sum_{m=0}^{\infty} i m P_{i j k l m} \sum_{i_{1}=1}^{\infty} \sum_{j_{1}=0}^{\infty} \sum_{k_{1}=0}^{\infty} \sum_{l_{1}=0}^{\infty} \sum_{m_{1}=0}^{\infty} k_{1} P_{i_{1} j_{1} k_{1} l_{1} m_{1}}
\end{aligned}
$$

$\Rightarrow \frac{d \mu_{10001}}{d t}=k_{p} C_{M} \mu_{00101}+k_{p} C_{M} \mu_{00002}+k_{t r t} \mu_{10010} \mu_{00100}-k_{t r p} \mu_{10001} \mu_{10000}+k_{t r p} \mu_{20000} \mu_{00001}$

$-k_{r} \mu_{10001}-k_{t c} \mu_{10001} \mu_{00001}-\frac{1}{2} k_{t c} \mu_{10100} \mu_{00001}-\frac{1}{2} k_{t c} \mu_{10001} \mu_{00100}$ 
3.3.5 Second moment w.r.t. the number of active (uncapped) radicals and unrelaxed (just uncapped) radicals

$$
\begin{aligned}
& \frac{d \mu_{00101}}{d t}=\sum_{i=1}^{\infty} \sum_{j=0}^{\infty} \sum_{k=0}^{\infty} \sum_{l=0}^{\infty} \sum_{m=0}^{\infty} k m \frac{d P_{i j k l m}}{d t} \\
& =-k_{p} C_{M} \sum_{i=1}^{\infty} \sum_{j=0}^{\infty} \sum_{k=0}^{\infty} \sum_{l=0}^{\infty} \sum_{m=0}^{\infty} k^{2} m P_{i j k l m} \\
& +k_{p} C_{M} \sum_{i=2}^{\infty} \sum_{j=0}^{\infty} \sum_{k=0}^{\infty} \sum_{l=0}^{\infty} \sum_{m=0}^{\infty} k^{2} m P_{i-1 j k l m} \\
& -k_{p} C_{M} \sum_{i=1}^{\infty} \sum_{j=0}^{\infty} \sum_{k=0}^{\infty} \sum_{l=0}^{\infty} \sum_{m=0}^{\infty} k m^{2} P_{i j k l m} \\
& +k_{p} C_{M} \sum_{i=2}^{\infty} \sum_{j=0}^{\infty} \sum_{k=0}^{\infty} \sum_{l=0}^{\infty} \sum_{m=0}^{\infty} k m^{2} P_{i-1 j k l m} \\
& -k_{t r t} C_{T} \sum_{i=1}^{\infty} \sum_{j=0}^{\infty} \sum_{k=0}^{\infty} \sum_{l=0}^{\infty} \sum_{m=0}^{\infty} k^{2} m P_{i j k l m} \\
& +k_{t r t} C_{T} \sum_{i=1}^{\infty} \sum_{j=0}^{\infty} \sum_{k=0}^{\infty} \sum_{l=1}^{\infty} \sum_{m=0}^{\infty} k(k+1) m P_{i j k+1 l-1 m} \\
& -k_{t r t} \sum_{i=1}^{\infty} \sum_{j=0}^{\infty} \sum_{k=0}^{\infty} \sum_{l=0}^{\infty} \sum_{m=0}^{\infty} k^{2} m P_{i j k l m} \sum_{i_{1}=1}^{\infty} \sum_{j_{1}=0}^{\infty} \sum_{k_{1}=0}^{\infty} \sum_{l_{1}=0}^{\infty} \sum_{m_{1}=0}^{\infty} l_{1} P_{i_{1} j_{1} k_{1} l_{1} m_{1}} \\
& -k_{t r t} \sum_{i=1}^{\infty} \sum_{j=0}^{\infty} \sum_{k=0}^{\infty} \sum_{l=0}^{\infty} \sum_{m=0}^{\infty} k l m P_{i j k l m} \sum_{i_{1}=1}^{\infty} \sum_{j_{1}=0}^{\infty} \sum_{k_{1}=0}^{\infty} \sum_{l_{1}=0}^{\infty} \sum_{m_{1}=0}^{\infty} k_{1} P_{i_{1} j_{1} k_{1} l_{1} m_{1}} \\
& +k_{t r t} \sum_{i=1}^{\infty} \sum_{j=0}^{\infty} \sum_{k=0}^{\infty} \sum_{l=1}^{\infty} \sum_{m=0}^{\infty} k(k+1) m P_{i j k+1 l-1 m} \sum_{i_{1}=1}^{\infty} \sum_{j_{1}=0}^{\infty} \sum_{k_{1}=0}^{\infty} \sum_{l_{1}=0}^{\infty} \sum_{m_{1}=0}^{\infty} l_{1} P_{i_{1} j_{1} k_{1} l_{1} m_{1}} \\
& +k_{t r t} \sum_{i=1}^{\infty} \sum_{j=0}^{\infty} \sum_{k=0}^{\infty} \sum_{l=0}^{\infty} \sum_{m=1}^{\infty} k(l+1) m P_{i j k l+1 m-1} \sum_{i_{1}=1}^{\infty} \sum_{j_{1}=0}^{\infty} \sum_{k_{1}=0}^{\infty} \sum_{l_{1}=0}^{\infty} \sum_{m_{1}=0}^{\infty} k_{1} P_{i_{1} j_{1} k_{1} l_{1} m_{1}} \\
& -k_{t r p} \sum_{i=1}^{\infty} \sum_{j=0}^{\infty} \sum_{k=0}^{\infty} \sum_{l=0}^{\infty} \sum_{m=0}^{\infty} k^{2} m P_{i j k l m} \sum_{i_{1}=1}^{\infty} \sum_{j_{1}=0}^{\infty} \sum_{k_{1}=0}^{\infty} \sum_{l_{1}=0}^{\infty} \sum_{m_{1}=0}^{\infty} i_{1} P_{i_{1} j_{1} k_{1} l_{1} m_{1}} \\
& -k_{t r p} \sum_{i=1}^{\infty} \sum_{j=0}^{\infty} \sum_{k=0}^{\infty} \sum_{l=0}^{\infty} \sum_{m=0}^{\infty} i k m P_{i j k l m} \sum_{i_{1}=1}^{\infty} \sum_{j_{1}=0}^{\infty} \sum_{k_{1}=0}^{\infty} \sum_{l_{1}=0}^{\infty} \sum_{m_{1}=0}^{\infty} k_{1} P_{i_{1} j_{1} k_{1} l_{1} m_{1}} \\
& +k_{t r p} \sum_{i=1}^{\infty} \sum_{j=0}^{\infty} \sum_{k=0}^{\infty} \sum_{l=0}^{\infty} \sum_{m=0}^{\infty} k(k+1) m P_{i j k+1 l m} \sum_{i_{1}=1}^{\infty} \sum_{j_{1}=0}^{\infty} \sum_{k_{1}=0}^{\infty} \sum_{l_{1}=0}^{\infty} \sum_{m_{1}=0}^{\infty} i_{1} P_{i_{1} j_{1} k_{1} l_{1} m_{1}} \\
& +k_{t r p} \sum_{i=1}^{\infty} \sum_{j=1}^{\infty} \sum_{k=1}^{\infty} \sum_{l=0}^{\infty} \sum_{m=0}^{\infty} i k m P_{i j-1 k-1 l m} \sum_{i_{1}=1}^{\infty} \sum_{j_{1}=0}^{\infty} \sum_{k_{1}=0}^{\infty} \sum_{l_{1}=0}^{\infty} \sum_{m_{1}=0}^{\infty} k_{1} P_{i_{1} j_{1} k_{1} l_{1} m_{1}}
\end{aligned}
$$


$-k_{\operatorname{trp}} \sum_{i=1}^{\infty} \sum_{j=0}^{\infty} \sum_{k=0}^{\infty} \sum_{l=0}^{\infty} \sum_{m=0}^{\infty} k m^{2} P_{i j k l m} \sum_{i_{1}=1}^{\infty} \sum_{j_{1}=0}^{\infty} \sum_{k_{1}=0}^{\infty} \sum_{l_{1}=0}^{\infty} \sum_{m_{1}=0}^{\infty} i_{1} P_{i_{1} j_{1} k_{1} l_{1} m_{1}}$

$-k_{t r p} \sum_{i=1}^{\infty} \sum_{j=0}^{\infty} \sum_{k=0}^{\infty} \sum_{l=0}^{\infty} \sum_{m=0}^{\infty} i k m P_{i j k l m} \sum_{i_{1}=1}^{\infty} \sum_{j_{1}=0}^{\infty} \sum_{k_{1}=0}^{\infty} \sum_{l_{1}=0}^{\infty} \sum_{m_{1}=0}^{\infty} m_{1} P_{i_{1} j_{1}} k_{1} l_{1} m_{1}$

$+k_{t r p} \sum_{i=1}^{\infty} \sum_{j=0}^{\infty} \sum_{k=0}^{\infty} \sum_{l=0}^{\infty} \sum_{m=0}^{\infty} k m(m+1) P_{i j k l m+1} \sum_{i_{1}=1}^{\infty} \sum_{j_{1}=0}^{\infty} \sum_{k_{1}=0}^{\infty} \sum_{l_{1}=0}^{\infty} \sum_{m_{1}=0}^{\infty} i_{1} P_{i_{1} j_{1}} k_{1} l_{1} m_{1}$

$+k_{t r p} \sum_{i=1}^{\infty} \sum_{j=1}^{\infty} \sum_{k=0}^{\infty} \sum_{l=0}^{\infty} \sum_{m=1}^{\infty} i k m P_{i j-1 k l m-1} \sum_{i_{1}=1}^{\infty} \sum_{j_{1}=0}^{\infty} \sum_{k_{1}=0}^{\infty} \sum_{l_{1}=0}^{\infty} \sum_{m_{1}=0}^{\infty} m_{1} P_{i_{1} j_{1} k_{1} l_{1} m_{1}}$

$-k_{\text {intr }} \sum_{i=1}^{\infty} \sum_{j=0}^{\infty} \sum_{k=0}^{\infty} \sum_{l=0}^{\infty} \sum_{m=0}^{\infty} k^{2} m P_{i j k l m}$

$+k_{i n t r} \sum_{i=1}^{\infty} \sum_{j=1}^{\infty} \sum_{k=0}^{\infty} \sum_{l=0}^{\infty} \sum_{m=0}^{\infty} k^{2} m P_{i j-1 k l m}$

$-k_{r} \sum_{i=1}^{\infty} \sum_{j=0}^{\infty} \sum_{k=0}^{\infty} \sum_{l=0}^{\infty} \sum_{m=0}^{\infty} k m^{2} P_{i j k l m}$

$+k_{r} \sum_{i=1}^{\infty} \sum_{j=0}^{\infty} \sum_{k=1}^{\infty} \sum_{l=0}^{\infty} \sum_{m=0}^{\infty} k m(m+1) P_{i j k-1 l m+1}$

$-k_{t c} \sum_{i=1}^{\infty} \sum_{j=0}^{\infty} \sum_{k=0}^{\infty} \sum_{l=0}^{\infty} \sum_{m=0}^{\infty} k^{2} m P_{i j k l m} \sum_{i_{1}=1}^{\infty} \sum_{j_{1}=0}^{\infty} \sum_{k_{1}=0}^{\infty} \sum_{l_{1}=0}^{\infty} \sum_{m_{1}=0}^{\infty} k_{1} P_{i_{1} j_{1} k_{1} l_{1} m_{1}}$

$+\frac{1}{2} k_{t c} \sum_{i=1}^{\infty} \sum_{j=0}^{\infty} \sum_{k=0}^{\infty} \sum_{l=0}^{\infty} \sum_{m=0}^{\infty} \sum_{i_{1}=1}^{i} \sum_{j_{1}=0}^{j} \sum_{k_{1}=0}^{k+2} \sum_{l_{1}=0}^{l} \sum_{m_{1}=0}^{m} k m\left[k_{1} P_{i_{1} j_{1} k_{1} l_{1} m_{1}}\left(k-k_{1}+2\right) P_{i-i_{1}, j-j_{1}, k-k_{1}+2, l-l_{1} m-m_{1}}\right]$

$-k_{t c} \sum_{i=1}^{\infty} \sum_{j=0}^{\infty} \sum_{k=0}^{\infty} \sum_{l=0}^{\infty} \sum_{m=0}^{\infty} k m^{2} P_{i j k l m} \sum_{i_{1}=1}^{\infty} \sum_{j_{1}=0}^{\infty} \sum_{k_{1}=0}^{\infty} \sum_{l_{1}=0}^{\infty} \sum_{m_{1}=0}^{\infty} m_{1} P_{i_{1} j_{1} k_{1} l_{1} m_{1}}$

$+\frac{1}{2} k_{t c} \sum_{i=1}^{\infty} \sum_{j=0}^{\infty} \sum_{k=0}^{\infty} \sum_{l=0}^{\infty} \sum_{m=0}^{\infty} \sum_{i_{1}=1}^{i} \sum_{j_{1}=0}^{j} \sum_{k_{1}=0}^{k} \sum_{l_{1}=0}^{l} \sum_{m_{1}=0}^{m+2}\left[k m m_{1} P_{i_{1} j_{1} k_{1} l_{1} m_{1}}\left(m+2-m_{1}\right) P_{i-i_{1}, j-j_{1}, k-k_{1}, l-l_{1} m+2-m_{1}}\right]$

$-k_{t c} \sum_{i=1}^{\infty} \sum_{j=0}^{\infty} \sum_{k=0}^{\infty} \sum_{l=0}^{\infty} \sum_{m=0}^{\infty} k^{2} m P_{i j k l m} \sum_{i_{1}=1}^{\infty} \sum_{j_{1}=0}^{\infty} \sum_{k_{1}=0}^{\infty} \sum_{l_{1}=0}^{\infty} \sum_{m_{1}=0}^{\infty} m_{1} P_{i_{1} j_{1} k_{1} l_{1} m_{1}}$

$-k_{t c} \sum_{i=1}^{\infty} \sum_{j=0}^{\infty} \sum_{k=0}^{\infty} \sum_{l=0}^{\infty} \sum_{m=0}^{\infty} k m^{2} P_{i j k l m} \sum_{i_{1}=1}^{\infty} \sum_{j_{1}=0}^{\infty} \sum_{k_{1}=0}^{\infty} \sum_{l_{1}=0}^{\infty} \sum_{m_{1}=0}^{\infty} k_{1} P_{i_{1} j_{1} k_{1} l_{1} m_{1}}$

$+\frac{1}{2} k_{t c} \sum_{i=1}^{\infty} \sum_{j=0}^{\infty} \sum_{k=0}^{\infty} \sum_{l=0}^{\infty} \sum_{m=0}^{\infty} \sum_{i_{1}=1}^{i} \sum_{j_{1}=0}^{j} \sum_{k_{1}=0}^{k+1} \sum_{l_{1}=0}^{l} \sum_{m_{1}=0}^{m+1}\left[k m k_{1} P_{i_{1} j_{1} k_{1} l_{1} m_{1}}\left(m+1-m_{1}\right) P_{i-i_{1}, j-j_{1}, k+1-k_{1}, l-l_{1} m+1-m_{1}}\right]$

$+\frac{1}{2} k_{t c} \sum_{i=1}^{\infty} \sum_{j=0}^{\infty} \sum_{k=0}^{\infty} \sum_{l=0}^{\infty} \sum_{m=0}^{\infty} \sum_{i_{1}=1}^{i} \sum_{j_{1}=0}^{j} \sum_{k_{1}=0}^{k+1} \sum_{l_{1}=0}^{l} \sum_{m_{1}=0}^{m+1}\left[k m m_{1} P_{i_{1} j_{1} k_{1} l_{1} m_{1}}\left(k+1-k_{1}\right) P_{i-i_{1}, j-j_{1}, k+1-k_{1}, l-l_{1} m+1-m_{1}}\right]$ 
Now

$$
\begin{aligned}
\sum_{k=0}^{\infty} \sum_{k_{1}=0}^{k+1} & \sum_{m=0}^{\infty} \sum_{m_{1}=0}^{m+1} k m k_{1} P_{k_{1}, m_{1}}\left(m+1-m_{1}\right) P_{k+1-k_{1}, m+1-m_{1}} \\
= & \sum_{k=0}^{\infty} \sum_{k_{1}=0}^{k+1} \sum_{m=0}^{\infty} \sum_{m_{1}=0}^{m+1}\left[k_{1}+\left(k-k_{1}\right)\right]\left[m_{1}+\left(m-m_{1}\right)\right] k_{1} P_{k_{1}, m_{1}}\left(m+1-m_{1}\right) P_{k+1-k_{1}, m+1-m_{1}} \\
= & \sum_{a=0}^{1}\left(\begin{array}{l}
1 \\
a
\end{array}\right) \sum_{k=0}^{\infty} \sum_{k_{1}=0}^{k+1} \sum_{m=0}^{\infty} \sum_{m_{1}=0}^{m+1} k_{1}^{1+a} m_{1}^{a} P_{k_{1}, m_{1}}\left(k+1-k_{1}-1\right)^{1-a} \\
& \times\left(m+1-m_{1}-1\right)^{1-a}\left(m+1-m_{1}\right) P_{k+1-k_{1}, m+1-m_{1}} \\
= & \sum_{a=0}^{1}\left(\begin{array}{l}
1 \\
a
\end{array}\right) \sum_{k=0}^{\infty} \sum_{m=0}^{\infty} k^{1+a} m^{a} P_{k, m} \sum_{k_{1}=0}^{\infty} \sum_{m_{1}=0}^{\infty}\left(k_{1}-1\right)^{1-a}\left(m_{1}-1\right)^{1-a} m_{1} P_{k_{1}, m_{1}} \\
= & \sum_{a=0}^{1}\left(\begin{array}{l}
1 \\
a
\end{array}\right) \mu_{1+a, a} \sum_{k_{1}=0}^{\infty} \sum_{m_{1}=0}^{\infty}\left(k_{1}-1\right)^{1-a}\left(m_{1}-1\right)^{1-a} m_{1} P_{k_{1}, m_{1}} \\
= & \left(\frac{1 !}{0 ! 1 !} \mu_{10} \sum_{k_{1}=0}^{\infty} \sum_{m_{1}=0}^{\infty}\left(k_{1}-1\right)\left(m_{1}-1\right) m_{1} P_{k_{1}, m_{1}}\right)+\left(\frac{1 !}{1 ! 0 !} \mu_{21} \sum_{k_{1}=0}^{\infty} \sum_{m_{1}=0}^{\infty} m_{1} P_{k_{1}, m_{1}}\right) \\
= & \mu_{10}\left(\mu_{12}-\mu_{11}-\mu_{02}+\mu_{01}\right)+\mu_{21} \mu_{01} \\
= & \mu_{21} \mu_{01}+\mu_{12} \mu_{10}+\mu_{10}\left(\mu_{01}-\mu_{11}-\mu_{02}\right)
\end{aligned}
$$

Similarly,

$$
\begin{aligned}
\sum_{k=0}^{\infty} \sum_{k_{1}=0}^{k+1} & \sum_{m=0}^{\infty} \sum_{m_{1}=0}^{m+1} k m m_{1} P_{k_{1}, m_{1}}\left(k+1-k_{1}\right) P_{k+1-k_{1}, m+1-m_{1}} \\
= & \sum_{k=0}^{\infty} \sum_{k_{1}=0}^{k+1} \sum_{m=0}^{\infty} \sum_{m_{1}=0}^{m+1}\left[k_{1}+\left(k-k_{1}\right)\right]\left[m_{1}+\left(m-m_{1}\right)\right] m_{1} P_{k_{1}, m_{1}}\left(k+1-k_{1}\right) P_{k+1-k_{1}, m+1-m_{1}} \\
= & \sum_{a=0}^{1}\left(\begin{array}{l}
1 \\
a
\end{array}\right) \sum_{k=0}^{\infty} \sum_{k_{1}=0}^{k+1} \sum_{m=0}^{\infty} \sum_{m_{1}=0}^{m+1} k_{1}^{a} m_{1}^{1+a} P_{k_{1}, m_{1}}\left(k+1-k_{1}-1\right)^{1-a} \\
& \times\left(m+1-m_{1}-1\right)^{1-a}\left(k+1-k_{1}\right) P_{k+1-k_{1}, m+1-m_{1}} \\
= & \sum_{a=0}^{1}\left(\begin{array}{l}
1 \\
a
\end{array}\right) \sum_{k=0}^{\infty} \sum_{m=0}^{\infty} k^{a} m^{1+a} P_{k, m} \sum_{k_{1}=0}^{\infty} \sum_{m_{1}=0}^{\infty}\left(k_{1}-1\right)^{1-a}\left(m_{1}-1\right)^{1-a} k_{1} P_{k_{1}, m_{1}} \\
= & \sum_{a=0}^{1}\left(\begin{array}{l}
1 \\
a
\end{array}\right) \mu_{a, 1+a} \sum_{k_{1}=0}^{\infty} \sum_{m_{1}=0}^{\infty}\left(k_{1}-1\right)^{1-a}\left(m_{1}-1\right)^{1-a} k_{1} P_{k_{1}, m_{1}} \\
= & \left(\frac{1 !}{0 ! 1 !} \mu_{01} \sum_{k_{1}=0}^{\infty} \sum_{m_{1}=0}^{\infty}\left(k_{1}-1\right)\left(m_{1}-1\right) k_{1} P_{k_{1}, m_{1}}\right)+\left(\frac{1 !}{1 ! 0 !} \mu_{12} \sum_{k_{1}=0}^{\infty} \sum_{m_{1}=0}^{\infty} k_{1} P_{k_{1}, m_{1}}\right) \\
= & \mu_{01}\left(\mu_{21}-\mu_{20}-\mu_{11}+\mu_{10}\right)+\mu_{12} \mu_{10} \\
= & \mu_{21} \mu_{01}+\mu_{12} \mu_{10}+\mu_{01}\left(\mu_{10}-\mu_{11}-\mu_{20}\right)
\end{aligned}
$$




$$
\begin{aligned}
& \Rightarrow \frac{d \mu_{00101}}{d t}=-k_{p} C_{M} \sum_{i=1}^{\infty} \sum_{j=0}^{\infty} \sum_{k=0}^{\infty} \sum_{l=0}^{\infty} \sum_{m=0}^{\infty} k^{2} m P_{i j k l m} \\
& +k_{p} C_{M} \sum_{i=1}^{\infty} \sum_{j=0}^{\infty} \sum_{k=0}^{\infty} \sum_{l=0}^{\infty} \sum_{m=0}^{\infty} k^{2} m P_{i j k l m} \\
& -k_{p} C_{M} \sum_{i=1}^{\infty} \sum_{j=0}^{\infty} \sum_{k=0}^{\infty} \sum_{l=0}^{\infty} \sum_{m=0}^{\infty} k m^{2} P_{i j k l m} \\
& +k_{p} C_{M} \sum_{i=1}^{\infty} \sum_{j=0}^{\infty} \sum_{k=0}^{\infty} \sum_{l=0}^{\infty} \sum_{m=0}^{\infty} k m^{2} P_{i j k l m} \\
& -k_{t r t} C_{T} \sum_{i=1}^{\infty} \sum_{j=0}^{\infty} \sum_{k=0}^{\infty} \sum_{l=0}^{\infty} \sum_{m=0}^{\infty} k^{2} m P_{i j k l m} \\
& +k_{t r t} C_{T} \sum_{i=1}^{\infty} \sum_{j=0}^{\infty} \sum_{k=0}^{\infty} \sum_{l=0}^{\infty} \sum_{m=0}^{\infty}(k-1) k m P_{i j k l m} \\
& -k_{t r t} \sum_{i=1}^{\infty} \sum_{j=0}^{\infty} \sum_{k=0}^{\infty} \sum_{l=0}^{\infty} \sum_{m=0}^{\infty} k^{2} m P_{i j k l m} \sum_{i_{1}=1}^{\infty} \sum_{j_{1}=0}^{\infty} \sum_{k_{1}=0}^{\infty} \sum_{l_{1}=0}^{\infty} \sum_{m_{1}=0}^{\infty} l_{1} P_{i_{1} j_{1} k_{1} l_{1} m_{1}} \\
& -k_{t r t} \sum_{i=1}^{\infty} \sum_{j=0}^{\infty} \sum_{k=0}^{\infty} \sum_{l=0}^{\infty} \sum_{m=0}^{\infty} k l m P_{i j k l m} \sum_{i_{1}=1}^{\infty} \sum_{j_{1}=0}^{\infty} \sum_{k_{1}=0}^{\infty} \sum_{l_{1}=0}^{\infty} \sum_{m_{1}=0}^{\infty} k_{1} P_{i_{1} j_{1} k_{1} l_{1} m_{1}} \\
& +k_{t r t} \sum_{i=1}^{\infty} \sum_{j=0}^{\infty} \sum_{k=0}^{\infty} \sum_{l=0}^{\infty} \sum_{m=0}^{\infty}(k-1) k m P_{i j k l m} \sum_{i_{1}=1}^{\infty} \sum_{j_{1}=0}^{\infty} \sum_{k_{1}=0}^{\infty} \sum_{l_{1}=0}^{\infty} \sum_{m_{1}=0}^{\infty} l_{1} P_{i_{1} j_{1}} k_{1} l_{1} m_{1} \\
& +k_{\text {trt }} \sum_{i=1}^{\infty} \sum_{j=0}^{\infty} \sum_{k=0}^{\infty} \sum_{l=0}^{\infty} \sum_{m=0}^{\infty} k l(m+1) P_{i j k l m} \sum_{i_{1}=1}^{\infty} \sum_{j_{1}=0}^{\infty} \sum_{k_{1}=0}^{\infty} \sum_{l_{1}=0}^{\infty} \sum_{m_{1}=0}^{\infty} k_{1} P_{i_{1} j_{1} k_{1} l_{1} m_{1}} \\
& -k_{t r p} \sum_{i=1}^{\infty} \sum_{j=0}^{\infty} \sum_{k=0}^{\infty} \sum_{l=0}^{\infty} \sum_{m=0}^{\infty} k^{2} m P_{i j k l m} \sum_{i_{1}=1}^{\infty} \sum_{j_{1}=0}^{\infty} \sum_{k_{1}=0}^{\infty} \sum_{l_{1}=0}^{\infty} \sum_{m_{1}=0}^{\infty} i_{1} P_{i_{1} j_{1} k_{1} l_{1} m_{1}} \\
& -k_{t r p} \sum_{i=1}^{\infty} \sum_{j=0}^{\infty} \sum_{k=0}^{\infty} \sum_{l=0}^{\infty} \sum_{m=0}^{\infty} i k m P_{i j k l m} \sum_{i_{1}=1}^{\infty} \sum_{j_{1}=0}^{\infty} \sum_{k_{1}=0}^{\infty} \sum_{l_{1}=0}^{\infty} \sum_{m_{1}=0}^{\infty} k_{1} P_{i_{1} j_{1} k_{1} l_{1} m_{1}} \\
& +k_{\text {trp }} \sum_{i=1}^{\infty} \sum_{j=0}^{\infty} \sum_{k=0}^{\infty} \sum_{l=0}^{\infty} \sum_{m=0}^{\infty}(k-1) k m P_{i j k l m} \sum_{i_{1}=1}^{\infty} \sum_{j_{1}=0}^{\infty} \sum_{k_{1}=0}^{\infty} \sum_{l_{1}=0}^{\infty} \sum_{m_{1}=0}^{\infty} i_{1} P_{i_{1} j_{1} k_{1} l_{1} m_{1}} \\
& +k_{t r p} \sum_{i=1}^{\infty} \sum_{j=0}^{\infty} \sum_{k=0}^{\infty} \sum_{l=0}^{\infty} \sum_{m=0}^{\infty} i(k+1) m P_{i j k l m} \sum_{i_{1}=1}^{\infty} \sum_{j_{1}=0}^{\infty} \sum_{k_{1}=0}^{\infty} \sum_{l_{1}=0}^{\infty} \sum_{m_{1}=0}^{\infty} k_{1} P_{i_{1} j_{1} k_{1} l_{1} m_{1}}
\end{aligned}
$$




$$
\begin{aligned}
& -k_{t r p} \sum_{i=1}^{\infty} \sum_{j=0}^{\infty} \sum_{k=0}^{\infty} \sum_{l=0}^{\infty} \sum_{m=0}^{\infty} k m^{2} P_{i j k l m} \sum_{i_{1}=1}^{\infty} \sum_{j_{1}=0}^{\infty} \sum_{k_{1}=0}^{\infty} \sum_{l_{1}=0}^{\infty} \sum_{m_{1}=0}^{\infty} i_{1} P_{i_{1} j_{1} k_{1} l_{1} m_{1}} \\
& -k_{t r p} \sum_{i=1}^{\infty} \sum_{j=0}^{\infty} \sum_{k=0}^{\infty} \sum_{l=0}^{\infty} \sum_{m=0}^{\infty} i k m P_{i j k l m} \sum_{i_{1}=1}^{\infty} \sum_{j_{1}=0}^{\infty} \sum_{k_{1}=0}^{\infty} \sum_{l_{1}=0}^{\infty} \sum_{m_{1}=0}^{\infty} m_{1} P_{i_{1} j_{1} k_{1} l_{1} m_{1}} \\
& +k_{\text {trp }} \sum_{i=1}^{\infty} \sum_{j=0}^{\infty} \sum_{k=0}^{\infty} \sum_{l=0}^{\infty} \sum_{m=0}^{\infty} k(m-1) m P_{i j k l m} \sum_{i_{1}=1}^{\infty} \sum_{j_{1}=0}^{\infty} \sum_{k_{1}=0}^{\infty} \sum_{l_{1}=0}^{\infty} \sum_{m_{1}=0}^{\infty} i_{1} P_{i_{1} j_{1} k_{1} l_{1} m_{1}} \\
& +k_{\operatorname{trp}} \sum_{i=1}^{\infty} \sum_{j=0}^{\infty} \sum_{k=0}^{\infty} \sum_{l=0}^{\infty} \sum_{m=0}^{\infty} i k(m+1) P_{i j k l m} \sum_{i_{1}=1}^{\infty} \sum_{j_{1}=0}^{\infty} \sum_{k_{1}=0}^{\infty} \sum_{l_{1}=0}^{\infty} \sum_{m_{1}=0}^{\infty} m_{1} P_{i_{1} j_{1} k_{1} l_{1} m_{1}} \\
& -k_{i n t r} \sum_{i=1}^{\infty} \sum_{j=0}^{\infty} \sum_{k=0}^{\infty} \sum_{l=0}^{\infty} \sum_{m=0}^{\infty} k^{2} m P_{i j k l m} \\
& +k_{i n t r} \sum_{i=1}^{\infty} \sum_{j=0}^{\infty} \sum_{k=0}^{\infty} \sum_{l=0}^{\infty} \sum_{m=0}^{\infty} k^{2} m P_{i j k l m} \\
& -k_{r} \sum_{i=1}^{\infty} \sum_{j=0}^{\infty} \sum_{k=0}^{\infty} \sum_{l=0}^{\infty} \sum_{m=0}^{\infty} k m^{2} P_{i j k l m} \\
& +k_{r} \sum_{i=1}^{\infty} \sum_{j=0}^{\infty} \sum_{k=0}^{\infty} \sum_{l=0}^{\infty} \sum_{m=0}^{\infty}(k+1)(m-1) m P_{i j k l m} \\
& -k_{t c} \sum_{i=1}^{\infty} \sum_{j=0}^{\infty} \sum_{k=0}^{\infty} \sum_{l=0}^{\infty} \sum_{m=0}^{\infty} k^{2} m P_{i j k l m} \sum_{i_{1}=1}^{\infty} \sum_{j_{1}=0}^{\infty} \sum_{k_{1}=0}^{\infty} \sum_{l_{1}=0}^{\infty} \sum_{m_{1}=0}^{\infty} k_{1} P_{i_{1} j_{1} k_{1} l_{1} m_{1}} \\
& +\frac{1}{2}(2) k_{t c} \sum_{i=1}^{\infty} \sum_{j=0}^{\infty} \sum_{k=0}^{\infty} \sum_{l=0}^{\infty} \sum_{m=0}^{\infty} k^{2} m P_{i j k l m} \sum_{i_{1}=1}^{\infty} \sum_{j_{1}=0}^{\infty} \sum_{k_{1}=0}^{\infty} \sum_{l_{1}=0}^{\infty} \sum_{m_{1}=0}^{\infty} k_{1} P_{i_{1} j_{1} k_{1} l_{1} m_{1}} \\
& -\frac{1}{2}(2) k_{t c} \sum_{i=1}^{\infty} \sum_{j=0}^{\infty} \sum_{k=0}^{\infty} \sum_{l=0}^{\infty} \sum_{m=0}^{\infty} k m P_{i j k l m} \sum_{i_{1}=1}^{\infty} \sum_{j_{1}=0}^{\infty} \sum_{k_{1}=0}^{\infty} \sum_{l_{1}=0}^{\infty} \sum_{m_{1}=0}^{\infty} k_{1} P_{i_{1} j_{1} k_{1} l_{1} m_{1}} \\
& -k_{t c} \sum_{i=1}^{\infty} \sum_{j=0}^{\infty} \sum_{k=0}^{\infty} \sum_{l=0}^{\infty} \sum_{m=0}^{\infty} k m^{2} P_{i j k l m} \sum_{i_{1}=1}^{\infty} \sum_{j_{1}=0}^{\infty} \sum_{k_{1}=0}^{\infty} \sum_{l_{1}=0}^{\infty} \sum_{m_{1}=0}^{\infty} m_{1} P_{i_{1} j_{1} k_{1} l_{1} m_{1}} \\
& +\frac{1}{2}(2) k_{t c} \sum_{i=1}^{\infty} \sum_{j=0}^{\infty} \sum_{k=0}^{\infty} \sum_{l=0}^{\infty} \sum_{m=0}^{\infty} k m^{2} P_{i j k l m} \sum_{i_{1}=1}^{\infty} \sum_{j_{1}=0}^{\infty} \sum_{k_{1}=0}^{\infty} \sum_{l_{1}=0}^{\infty} \sum_{m_{1}=0}^{\infty} m_{1} P_{i_{1} j_{1} k_{1} l_{1} m_{1}} \\
& -\frac{1}{2}(2) k_{t c} \sum_{i=1}^{\infty} \sum_{j=0}^{\infty} \sum_{k=0}^{\infty} \sum_{l=0}^{\infty} \sum_{m=0}^{\infty} k m P_{i j k l m} \sum_{i_{1}=1}^{\infty} \sum_{j_{1}=0}^{\infty} \sum_{k_{1}=0}^{\infty} \sum_{l_{1}=0}^{\infty} \sum_{m_{1}=0}^{\infty} m_{1} P_{i_{1} j_{1} k_{1} l_{1} m_{1}} \\
& -k_{t c} \sum_{i=1}^{\infty} \sum_{j=0}^{\infty} \sum_{k=0}^{\infty} \sum_{l=0}^{\infty} \sum_{m=0}^{\infty} k^{2} m P_{i j k l m} \sum_{i_{1}=1}^{\infty} \sum_{j_{1}=0}^{\infty} \sum_{k_{1}=0}^{\infty} \sum_{l_{1}=0}^{\infty} \sum_{m_{1}=0}^{\infty} m_{1} P_{i_{1} j_{1} k_{1} l_{1} m_{1}} \\
& -k_{t c} \sum_{i=1}^{\infty} \sum_{j=0}^{\infty} \sum_{k=0}^{\infty} \sum_{l=0}^{\infty} \sum_{m=0}^{\infty} k m^{2} P_{i j k l m} \sum_{i_{1}=1}^{\infty} \sum_{j_{1}=0}^{\infty} \sum_{k_{1}=0}^{\infty} \sum_{l_{1}=0}^{\infty} \sum_{m_{1}=0}^{\infty} k_{1} P_{i_{1} j_{1} k_{1} l_{1} m_{1}}
\end{aligned}
$$




$$
\begin{aligned}
& +\frac{1}{2}(2) k_{t c} \sum_{i=1}^{\infty} \sum_{j=0}^{\infty} \sum_{k=0}^{\infty} \sum_{l=0}^{\infty} \sum_{m=0}^{\infty} k^{2} m P_{i j k l m} \sum_{i_{1}=1}^{\infty} \sum_{j_{1}=0}^{\infty} \sum_{k_{1}=0}^{\infty} \sum_{l_{1}=0}^{\infty} \sum_{m_{1}=0}^{\infty} m_{1} P_{i_{1} j_{1} k_{1} l_{1} m_{1}} \\
& +\frac{1}{2}(2) k_{t c} \sum_{i=1}^{\infty} \sum_{j=0}^{\infty} \sum_{k=0}^{\infty} \sum_{l=0}^{\infty} \sum_{m=0}^{\infty} k m^{2} P_{i j k l m} \sum_{i_{1}=1}^{\infty} \sum_{j_{1}=0}^{\infty} \sum_{k_{1}=0}^{\infty} \sum_{l_{1}=0}^{\infty} \sum_{m_{1}=0}^{\infty} k_{1} P_{i_{1} j_{1} k_{1} l_{1} m_{1}} \\
& +\frac{1}{2}(2) k_{t c} \sum_{i=1}^{\infty} \sum_{j=0}^{\infty} \sum_{k=0}^{\infty} \sum_{l=0}^{\infty} \sum_{m=0}^{\infty} k P_{i j k l m} \sum_{i_{1}=1}^{\infty} \sum_{j_{1}=0}^{\infty} \sum_{k_{1}=0}^{\infty} \sum_{l_{1}=0}^{\infty} \sum_{m_{1}=0}^{\infty} m_{1} P_{i_{1} j_{1} k_{1} l_{1} m_{1}} \\
& -\frac{1}{2} k_{t c} \sum_{i=1}^{\infty} \sum_{j=0}^{\infty} \sum_{k=0}^{\infty} \sum_{l=0}^{\infty} \sum_{m=0}^{\infty} k m P_{i j k l m} \sum_{i_{1}=1}^{\infty} \sum_{j_{1}=0}^{\infty} \sum_{k_{1}=0}^{\infty} \sum_{l_{1}=0}^{\infty} \sum_{m_{1}=0}^{\infty} k_{1} P_{i_{1} j_{1} k_{1} l_{1} m_{1}} \\
& -\frac{1}{2} k_{t c} \sum_{i=1}^{\infty} \sum_{j=0}^{\infty} \sum_{k=0}^{\infty} \sum_{l=0}^{\infty} \sum_{m=0}^{\infty} k m P_{i j k l m} \sum_{i_{1}=1}^{\infty} \sum_{j_{1}=0}^{\infty} \sum_{k_{1}=0}^{\infty} \sum_{l_{1}=0}^{\infty} \sum_{m_{1}=0}^{\infty} m_{1} P_{i_{1} j_{1} k_{1} l_{1} m_{1}} \\
& -\frac{1}{2} k_{t c} \sum_{i=1}^{\infty} \sum_{j=0}^{\infty} \sum_{k=0}^{\infty} \sum_{l=0}^{\infty} \sum_{m=0}^{\infty} k^{2} P_{i j k l m} \sum_{i_{1}=1}^{\infty} \sum_{j_{1}=0}^{\infty} \sum_{k_{1}=0}^{\infty} \sum_{l_{1}=0}^{\infty} \sum_{m_{1}=0}^{\infty} m_{1} P_{i_{1} j_{1} k_{1} l_{1} m_{1}} \\
& -\frac{1}{2} k_{t c} \sum_{i=1}^{\infty} \sum_{j=0}^{\infty} \sum_{k=0}^{\infty} \sum_{l=0}^{\infty} \sum_{m=0}^{\infty} m^{2} P_{i j k l m} \sum_{i_{1}=1}^{\infty} \sum_{j_{1}=0}^{\infty} \sum_{k_{1}=0}^{\infty} \sum_{l_{1}=0}^{\infty} \sum_{m_{1}=0}^{\infty} k_{1} P_{i_{1} j_{1} k_{1} l_{1} m_{1}}
\end{aligned}
$$

using Equations (73), (92) and (93).

$$
\begin{aligned}
& \Rightarrow \frac{d \mu_{00101}}{d t}=-k_{t r t} C_{T} \sum_{i=1}^{\infty} \sum_{j=0}^{\infty} \sum_{k=0}^{\infty} \sum_{l=0}^{\infty} \sum_{m=0}^{\infty} k m P_{i j k l m} \\
& \quad-k_{t r t} \sum_{i=1}^{\infty} \sum_{j=0}^{\infty} \sum_{k=0}^{\infty} \sum_{l=0}^{\infty} \sum_{m=0}^{\infty} k m P_{i j k l m} \sum_{i_{1}=1}^{\infty} \sum_{j_{1}=0}^{\infty} \sum_{k_{1}=0}^{\infty} \sum_{l_{1}=0}^{\infty} \sum_{m_{1}=0}^{\infty} l_{1} P_{i_{1} j_{1} k_{1} l_{1} m_{1}} \\
& \quad+k_{\text {trt }} \sum_{i=1}^{\infty} \sum_{j=0}^{\infty} \sum_{k=0}^{\infty} \sum_{l=0}^{\infty} \sum_{m=0}^{\infty} k l P_{i j k l m} \sum_{i_{1}=1}^{\infty} \sum_{j_{1}=0}^{\infty} \sum_{k_{1}=0}^{\infty} \sum_{l_{1}=0}^{\infty} \sum_{m_{1}=0}^{\infty} k_{1} P_{i_{1} j_{1} k_{1} l_{1} m_{1}} \\
& \quad-k_{\text {trp }} \sum_{i=1}^{\infty} \sum_{j=0}^{\infty} \sum_{k=0}^{\infty} \sum_{l=0}^{\infty} \sum_{m=0}^{\infty} k m P_{i j k l m} \sum_{i_{1}=1}^{\infty} \sum_{j_{1}=0}^{\infty} \sum_{k_{1}=0}^{\infty} \sum_{l_{1}=0}^{\infty} \sum_{m_{1}=0}^{\infty} i_{1} P_{i_{1} j_{1} k_{1} l_{1} m_{1}} \\
& +k_{\text {trp }} \sum_{i=1}^{\infty} \sum_{j=0}^{\infty} \sum_{k=0}^{\infty} \sum_{l=0}^{\infty} \sum_{m=0}^{\infty} i m P_{i j k l m} \sum_{i_{1}=1}^{\infty} \sum_{j_{1}=0}^{\infty} \sum_{k_{1}=0}^{\infty} \sum_{l_{1}=0}^{\infty} \sum_{m_{1}=0}^{\infty} k_{1} P_{i_{1} j_{1} k_{1} l_{1} m_{1}} \\
& \quad-k_{t r p} \sum_{i=1}^{\infty} \sum_{j=0}^{\infty} \sum_{k=0}^{\infty} \sum_{l=0}^{\infty} \sum_{m=0}^{\infty} k m P_{i j k l m} \sum_{i_{1}=1}^{\infty} \sum_{j_{1}=0}^{\infty} \sum_{k_{1}=0}^{\infty} \sum_{l_{1}=0}^{\infty} \sum_{m_{1}=0}^{\infty} i_{1} P_{i_{1} j_{1} k_{1} l_{1} m_{1}} \\
& \quad+k_{\text {trp }} \sum_{i=1}^{\infty} \sum_{j=0}^{\infty} \sum_{k=0}^{\infty} \sum_{l=0}^{\infty} \sum_{m=0}^{\infty} i k P_{i j k l m} \sum_{i_{1}=1}^{\infty} \sum_{j_{1}=0}^{\infty} \sum_{k_{1}=0}^{\infty} \sum_{l_{1}=0}^{\infty} \sum_{m_{1}=0}^{\infty} m_{1} P_{i_{1} j_{1} k_{1} l_{1} m_{1}} \\
& +k_{r} \sum_{i=1}^{\infty} \sum_{j=0}^{\infty} \sum_{k=0}^{\infty} \sum_{l=0}^{\infty} \sum_{m=0}^{\infty}\left(m^{2}-k m-m\right) P_{i j k l m}
\end{aligned}
$$




$$
\begin{aligned}
& -k_{t c} \sum_{i=1}^{\infty} \sum_{j=0}^{\infty} \sum_{k=0}^{\infty} \sum_{l=0}^{\infty} \sum_{m=0}^{\infty} k m P_{i j k l m} \sum_{i_{1}=1}^{\infty} \sum_{j_{1}=0}^{\infty} \sum_{k_{1}=0}^{\infty} \sum_{l_{1}=0}^{\infty} \sum_{m_{1}=0}^{\infty} k_{1} P_{i_{1} j_{1} k_{1} l_{1} m_{1}} \\
& -k_{t c} \sum_{i=1}^{\infty} \sum_{j=0}^{\infty} \sum_{k=0}^{\infty} \sum_{l=0}^{\infty} \sum_{m=0}^{\infty} k m P_{i j k l m} \sum_{i_{1}=1}^{\infty} \sum_{j_{1}=0}^{\infty} \sum_{k_{1}=0}^{\infty} \sum_{l_{1}=0}^{\infty} \sum_{m_{1}=0}^{\infty} m_{1} P_{i_{1} j_{1} k_{1} l_{1} m_{1}} \\
& +k_{t c} \sum_{i=1}^{\infty} \sum_{j=0}^{\infty} \sum_{k=0}^{\infty} \sum_{l=0}^{\infty} \sum_{m=0}^{\infty} k P_{i j k l m} \sum_{i_{1}=1}^{\infty} \sum_{j_{1}=0}^{\infty} \sum_{k_{1}=0}^{\infty} \sum_{l_{1}=0}^{\infty} \sum_{m_{1}=0}^{\infty} m_{1} P_{i_{1} j_{1} k_{1} l_{1} m_{1}} \\
& -\frac{1}{2} k_{t c} \sum_{i=1}^{\infty} \sum_{j=0}^{\infty} \sum_{k=0}^{\infty} \sum_{l=0}^{\infty} \sum_{m=0}^{\infty} k m P_{i j k l m} \sum_{i_{1}=1}^{\infty} \sum_{j_{1}=0}^{\infty} \sum_{k_{1}=0}^{\infty} \sum_{l_{1}=0}^{\infty} \sum_{m_{1}=0}^{\infty} k_{1} P_{i_{1} j_{1} k_{1} l_{1} m_{1}} \\
& -\frac{1}{2} k_{t c} \sum_{i=1}^{\infty} \sum_{j=0}^{\infty} \sum_{k=0}^{\infty} \sum_{l=0}^{\infty} \sum_{m=0}^{\infty} k m P_{i j k l m} \sum_{i_{1}=1}^{\infty} \sum_{j_{1}=0}^{\infty} \sum_{k_{1}=0}^{\infty} \sum_{l_{1}=0}^{\infty} \sum_{m_{1}=0}^{\infty} m_{1} P_{i_{1} j_{1} k_{1} l_{1} m_{1}} \\
& -\frac{1}{2} k_{t c} \sum_{i=1}^{\infty} \sum_{j=0}^{\infty} \sum_{k=0}^{\infty} \sum_{l=0}^{\infty} \sum_{m=0}^{\infty} k^{2} P_{i j k l m} \sum_{i_{1}=1}^{\infty} \sum_{j_{1}=0}^{\infty} \sum_{k_{1}=0}^{\infty} \sum_{l_{1}=0}^{\infty} \sum_{m_{1}=0}^{\infty} m_{1} P_{i_{1} j_{1} k_{1} l_{1} m_{1}} \\
& -\frac{1}{2} k_{t c} \sum_{i=1}^{\infty} \sum_{j=0}^{\infty} \sum_{k=0}^{\infty} \sum_{l=0}^{\infty} \sum_{m=0}^{\infty} m^{2} P_{i j k l m} \sum_{i_{1}=1}^{\infty} \sum_{j_{1}=0}^{\infty} \sum_{k_{1}=0}^{\infty} \sum_{l_{1}=0}^{\infty} \sum_{m_{1}=0}^{\infty} k_{1} P_{i_{1} j_{1} k_{1} l_{1} m_{1}} \\
& +\frac{d \mu_{00101}}{d t}=-k_{t r t} C_{T} \mu_{00101}-k_{t r t} \mu_{00101} \mu_{00010}+k_{t r t} \mu_{00110} \mu_{00100} \\
& -2 k_{t r p} \mu_{00101} \mu_{10000}+k_{t r p} \mu_{10001} \mu_{00100}+k_{t r p} \mu_{10100} \mu_{00001}+k_{r}\left(\mu_{00002}-\mu_{00101}-\mu_{00001}\right) \\
& +\frac{3}{2} k_{t c} \mu_{00101}\left(\mu_{000100}+\mu_{00001}\right)-\frac{1}{2} k_{t c} \mu_{00200} \mu_{00001}-\frac{1}{2} k_{t c} \mu_{00002} \mu_{00100}
\end{aligned}
$$


3.3.6 Second moment w.r.t. the number of repeat units and inactive (capped) radicals

$$
\begin{aligned}
& \frac{d \mu_{10010}}{d t}=\sum_{i=1}^{\infty} \sum_{j=0}^{\infty} \sum_{k=0}^{\infty} \sum_{l=0}^{\infty} \sum_{m=0}^{\infty} i l \frac{d P_{i j k l m}}{d t} \\
& =-k_{p} C_{M} \sum_{i=1}^{\infty} \sum_{j=0}^{\infty} \sum_{k=0}^{\infty} \sum_{l=0}^{\infty} \sum_{m=0}^{\infty} i k l P_{i j k l m} \\
& +k_{p} C_{M} \sum_{i=2}^{\infty} \sum_{j=0}^{\infty} \sum_{k=0}^{\infty} \sum_{l=0}^{\infty} \sum_{m=0}^{\infty} i k l P_{i-1 j k l m} \\
& -k_{p} C_{M} \sum_{i=1}^{\infty} \sum_{j=0}^{\infty} \sum_{k=0}^{\infty} \sum_{l=0}^{\infty} \sum_{m=0}^{\infty} i l m P_{i j k l m} \\
& +k_{p} C_{M} \sum_{i=2}^{\infty} \sum_{j=0}^{\infty} \sum_{k=0}^{\infty} \sum_{l=0}^{\infty} \sum_{m=0}^{\infty} i l m P_{i-1 j k l m} \\
& -k_{t r t} C_{T} \sum_{i=1}^{\infty} \sum_{j=0}^{\infty} \sum_{k=0}^{\infty} \sum_{l=0}^{\infty} \sum_{m=0}^{\infty} i k l P_{i j k l m} \\
& +k_{t r t} C_{T} \sum_{i=1}^{\infty} \sum_{j=0}^{\infty} \sum_{k=0}^{\infty} \sum_{l=1}^{\infty} \sum_{m=0}^{\infty} i(k+1) l P_{i j k+1 l-1 m} \\
& -k_{t r t} \sum_{i=1}^{\infty} \sum_{j=0}^{\infty} \sum_{k=0}^{\infty} \sum_{l=0}^{\infty} \sum_{m=0}^{\infty} i k l P_{i j k l m} \sum_{i_{1}=1}^{\infty} \sum_{j_{1}=0}^{\infty} \sum_{k_{1}=0}^{\infty} \sum_{l_{1}=0}^{\infty} \sum_{m_{1}=0}^{\infty} l_{1} P_{i_{1} j_{1} k_{1} l_{1} m_{1}} \\
& -k_{t r t} \sum_{i=1}^{\infty} \sum_{j=0}^{\infty} \sum_{k=0}^{\infty} \sum_{l=0}^{\infty} \sum_{m=0}^{\infty} i l^{2} P_{i j k l m} \sum_{i_{1}=1}^{\infty} \sum_{j_{1}=0}^{\infty} \sum_{k_{1}=0}^{\infty} \sum_{l_{1}=0}^{\infty} \sum_{m_{1}=0}^{\infty} k_{1} P_{i_{1} j_{1} k_{1} l_{1} m_{1}} \\
& +k_{t r t} \sum_{i=1}^{\infty} \sum_{j=0}^{\infty} \sum_{k=0}^{\infty} \sum_{l=1}^{\infty} \sum_{m=0}^{\infty} i(k+1) l P_{i j k+1 l-1 m} \sum_{i_{1}=1}^{\infty} \sum_{j_{1}=0}^{\infty} \sum_{k_{1}=0}^{\infty} \sum_{l_{1}=0}^{\infty} \sum_{m_{1}=0}^{\infty} l_{1} P_{i_{1} j_{1} k_{1} l_{1} m_{1}} \\
& +k_{t r t} \sum_{i=1}^{\infty} \sum_{j=0}^{\infty} \sum_{k=0}^{\infty} \sum_{l=0}^{\infty} \sum_{m=1}^{\infty} i l(l+1) P_{i j k l+1 m-1} \sum_{i_{1}=1}^{\infty} \sum_{j_{1}=0}^{\infty} \sum_{k_{1}=0}^{\infty} \sum_{l_{1}=0}^{\infty} \sum_{m_{1}=0}^{\infty} k_{1} P_{i_{1} j_{1} k_{1} l_{1} m_{1}} \\
& -k_{t r p} \sum_{i=1}^{\infty} \sum_{j=0}^{\infty} \sum_{k=0}^{\infty} \sum_{l=0}^{\infty} \sum_{m=0}^{\infty} i k l P_{i j k l m} \sum_{i_{1}=1}^{\infty} \sum_{j_{1}=0}^{\infty} \sum_{k_{1}=0}^{\infty} \sum_{l_{1}=0}^{\infty} \sum_{m_{1}=0}^{\infty} i_{1} P_{i_{1} j_{1} k_{1} l_{1} m_{1}} \\
& -k_{t r p} \sum_{i=1}^{\infty} \sum_{j=0}^{\infty} \sum_{k=0}^{\infty} \sum_{l=0}^{\infty} \sum_{m=0}^{\infty} i^{2} l P_{i j k l m} \sum_{i_{1}=1}^{\infty} \sum_{j_{1}=0}^{\infty} \sum_{k_{1}=0}^{\infty} \sum_{l_{1}=0}^{\infty} \sum_{m_{1}=0}^{\infty} k_{1} P_{i_{1} j_{1}} k_{1} l_{1} m_{1} \\
& +k_{\operatorname{trp}} \sum_{i=1}^{\infty} \sum_{j=0}^{\infty} \sum_{k=0}^{\infty} \sum_{l=0}^{\infty} \sum_{m=0}^{\infty} i(k+1) l P_{i j k+1 l m} \sum_{i_{1}=1}^{\infty} \sum_{j_{1}=0}^{\infty} \sum_{k_{1}=0}^{\infty} \sum_{l_{1}=0}^{\infty} \sum_{m_{1}=0}^{\infty} i_{1} P_{i_{1} j_{1} k_{1} l_{1} m_{1}} \\
& +k_{t r p} \sum_{i=1}^{\infty} \sum_{j=1}^{\infty} \sum_{k=1}^{\infty} \sum_{l=0}^{\infty} \sum_{m=0}^{\infty} i^{2} l P_{i j-1 k-1 l m} \sum_{i_{1}=1}^{\infty} \sum_{j_{1}=0}^{\infty} \sum_{k_{1}=0}^{\infty} \sum_{l_{1}=0}^{\infty} \sum_{m_{1}=0}^{\infty} k_{1} P_{i_{1} j_{1} k_{1} l_{1} m_{1}}
\end{aligned}
$$




$$
\begin{aligned}
& -k_{\operatorname{trp}} \sum_{i=1}^{\infty} \sum_{j=0}^{\infty} \sum_{k=0}^{\infty} \sum_{l=0}^{\infty} \sum_{m=0}^{\infty} i \operatorname{lm} P_{i j k l m} \sum_{i_{1}=1}^{\infty} \sum_{j_{1}=0}^{\infty} \sum_{k_{1}=0}^{\infty} \sum_{l_{1}=0}^{\infty} \sum_{m_{1}=0}^{\infty} i_{1} P_{i_{1} j_{1} k_{1} l_{1} m_{1}} \\
& -k_{\operatorname{trp}} \sum_{i=1}^{\infty} \sum_{j=0}^{\infty} \sum_{k=0}^{\infty} \sum_{l=0}^{\infty} \sum_{m=0}^{\infty} i^{2} l P_{i j k l m} \sum_{i_{1}=1}^{\infty} \sum_{j_{1}=0}^{\infty} \sum_{k_{1}=0}^{\infty} \sum_{l_{1}=0}^{\infty} \sum_{m_{1}=0}^{\infty} m_{1} P_{i_{1} j_{1} k_{1} l_{1} m_{1}} \\
& +k_{\text {trp }} \sum_{i=1}^{\infty} \sum_{j=0}^{\infty} \sum_{k=0}^{\infty} \sum_{l=0}^{\infty} \sum_{m=0}^{\infty} i l(m+1) P_{i j k l m+1} \sum_{i_{1}=1}^{\infty} \sum_{j_{1}=0}^{\infty} \sum_{k_{1}=0}^{\infty} \sum_{l_{1}=0}^{\infty} \sum_{m_{1}=0}^{\infty} i_{1} P_{i_{1} j_{1} k_{1} l_{1} m_{1}} \\
& +k_{t r p} \sum_{i=1}^{\infty} \sum_{j=1}^{\infty} \sum_{k=0}^{\infty} \sum_{l=0}^{\infty} \sum_{m=1}^{\infty} i^{2} l P_{i j-1 k l m-1} \sum_{i_{1}=1}^{\infty} \sum_{j_{1}=0}^{\infty} \sum_{k_{1}=0}^{\infty} \sum_{l_{1}=0}^{\infty} \sum_{m_{1}=0}^{\infty} m_{1} P_{i_{1} j_{1} k_{1} l_{1} m_{1}} \\
& -k_{i n t r} \sum_{i=1}^{\infty} \sum_{j=0}^{\infty} \sum_{k=0}^{\infty} \sum_{l=0}^{\infty} \sum_{m=0}^{\infty} i k l P_{i j k l m} \\
& +k_{i n t r} \sum_{i=1}^{\infty} \sum_{j=1}^{\infty} \sum_{k=0}^{\infty} \sum_{l=0}^{\infty} \sum_{m=0}^{\infty} i k l P_{i j-1 k l m} \\
& -k_{r} \sum_{i=1}^{\infty} \sum_{j=0}^{\infty} \sum_{k=0}^{\infty} \sum_{l=0}^{\infty} \sum_{m=0}^{\infty} i l m P_{i j k l m} \\
& +k_{r} \sum_{i=1}^{\infty} \sum_{j=0}^{\infty} \sum_{k=1}^{\infty} \sum_{l=0}^{\infty} \sum_{m=0}^{\infty} i l(m+1) P_{i j k-1 l m+1} \\
& -k_{t c} \sum_{i=1}^{\infty} \sum_{j=0}^{\infty} \sum_{k=0}^{\infty} \sum_{l=0}^{\infty} \sum_{m=0}^{\infty} i k l P_{i j k l m} \sum_{i_{1}=1}^{\infty} \sum_{j_{1}=0}^{\infty} \sum_{k_{1}=0}^{\infty} \sum_{l_{1}=0}^{\infty} \sum_{m_{1}=0}^{\infty} k_{1} P_{i_{1} j_{1} k_{1} l_{1} m_{1}} \\
& +\frac{1}{2} k_{t c} \sum_{i=1}^{\infty} \sum_{j=0}^{\infty} \sum_{k=0}^{\infty} \sum_{l=0}^{\infty} \sum_{m=0}^{\infty} \sum_{i_{1}=1}^{i} \sum_{j_{1}=0}^{j} \sum_{k_{1}=0}^{k+2} \sum_{l_{1}=0}^{l} \sum_{m_{1}=0}^{m}\left[i l k_{1} P_{i_{1} j_{1} k_{1} l_{1} m_{1}}\left(k+2-k_{1}\right) P_{i-i_{1}, j-j_{1}, k+2-k_{1}, l-l_{1} m-m_{1}}\right] \\
& -k_{t c} \sum_{i=1}^{\infty} \sum_{j=0}^{\infty} \sum_{k=0}^{\infty} \sum_{l=0}^{\infty} \sum_{m=0}^{\infty} i \operatorname{lm} P_{i j k l m} \sum_{i_{1}=1}^{\infty} \sum_{j_{1}=0}^{\infty} \sum_{k_{1}=0}^{\infty} \sum_{l_{1}=0}^{\infty} \sum_{m_{1}=0}^{\infty} m_{1} P_{i_{1} j_{1} k_{1} l_{1} m_{1}} \\
& +\frac{1}{2} k_{t c} \sum_{i=1}^{\infty} \sum_{j=0}^{\infty} \sum_{k=0}^{\infty} \sum_{l=0}^{\infty} \sum_{m=0}^{\infty} \sum_{i_{1}=1}^{i} \sum_{j_{1}=0}^{j} \sum_{k_{1}=0}^{k} \sum_{l_{1}=0}^{l} \sum_{m_{1}=0}^{m+2}\left[i l m_{1} P_{i_{1} j_{1} k_{1} l_{1} m_{1}}\left(m+2-m_{1}\right) P_{i-i_{1}, j-j_{1}, k-k_{1}, l-l_{1} m+2-m_{1}}\right] \\
& -k_{t c} \sum_{i=1}^{\infty} \sum_{j=0}^{\infty} \sum_{k=0}^{\infty} \sum_{l=0}^{\infty} \sum_{m=0}^{\infty} i k l P_{i j k l m} \sum_{i_{1}=1}^{\infty} \sum_{j_{1}=0}^{\infty} \sum_{k_{1}=0}^{\infty} \sum_{l_{1}=0}^{\infty} \sum_{m_{1}=0}^{\infty} m_{1} P_{i_{1} j_{1} k_{1} l_{1} m_{1}} \\
& -k_{t c} \sum_{i=1}^{\infty} \sum_{j=0}^{\infty} \sum_{k=0}^{\infty} \sum_{l=0}^{\infty} \sum_{m=0}^{\infty} i \operatorname{lm} P_{i j k l m} \sum_{i_{1}=1}^{\infty} \sum_{j_{1}=0}^{\infty} \sum_{k_{1}=0}^{\infty} \sum_{l_{1}=0}^{\infty} \sum_{m_{1}=0}^{\infty} k_{1} P_{i_{1} j_{1} k_{1} l_{1} m_{1}} \\
& +\frac{1}{2} k_{t c} \sum_{i=1}^{\infty} \sum_{j=0}^{\infty} \sum_{k=0}^{\infty} \sum_{l=0}^{\infty} \sum_{m=0}^{\infty} \sum_{i_{1}=1}^{i} \sum_{j_{1}=0}^{j} \sum_{k_{1}=0}^{k+1} \sum_{l_{1}=0}^{l} \sum_{m_{1}=0}^{m+1}\left[i l k_{1} P_{i_{1} j_{1} k_{1} l_{1} m_{1}}\left(m+1-m_{1}\right) P_{\left.i-i_{1}, j-j_{1}, k+1-k_{1}, l-l_{1} m+1-m_{1}\right]}\right] \\
& +\frac{1}{2} k_{t c} \sum_{i=1}^{\infty} \sum_{j=0}^{\infty} \sum_{k=0}^{\infty} \sum_{l=0}^{\infty} \sum_{m=0}^{\infty} \sum_{i_{1}=1}^{i} \sum_{j_{1}=0}^{j} \sum_{k_{1}=0}^{k+1} \sum_{l_{1}=0}^{l} \sum_{m_{1}=0}^{m+1}\left[i l m_{1} P_{i_{1} j_{1} k_{1} l_{1} m_{1}}\left(k+1-k_{1}\right) P_{i-i_{1}, j-j_{1}, k+1-k_{1}, l-l_{1} m+1-m_{1}}\right]
\end{aligned}
$$


Now

$$
\begin{aligned}
\sum_{i=1}^{\infty} \sum_{i_{1}=1}^{i} \sum_{k=0}^{\infty} \sum_{k_{1}=0}^{k+1} \sum_{l=0}^{\infty} \sum_{l_{1}=0}^{l} \sum_{m=0}^{\infty} \sum_{m_{1}=0}^{m+1} i l k_{1} P_{i_{1}, k_{1}, l_{1}, m_{1}}\left(m+1-m_{1}\right) P_{i-i_{1}, k+1-k_{1}, l-l_{1}, m+1-m_{1}} \\
=\sum_{i=1}^{\infty} \sum_{i_{1}=1}^{i} \sum_{k=0}^{\infty} \sum_{k_{1}=0}^{k+1} \sum_{l=0}^{\infty} \sum_{l_{1}=0}^{l} \sum_{m=0}^{\infty} \sum_{m_{1}=0}^{m+1}\left[i_{1}+\left(i-i_{1}\right)\right]\left[l_{1}+\left(l-l_{1}\right)\right] k_{1} P_{i_{1}, k_{1}, l_{1}, m_{1}} \\
\quad \times\left(m+1-m_{1}\right) P_{i-i_{1}, k+1-k_{1}, l-l_{1}, m+1-m_{1}} \\
=\sum_{a=0}^{1}\left(\begin{array}{l}
1 \\
a
\end{array}\right) \sum_{i=1}^{\infty} \sum_{i_{1}=1}^{i} \sum_{k=0}^{\infty} \sum_{k_{1}=0}^{k+1} \sum_{l=0}^{\infty} \sum_{l_{1}=0}^{l} \sum_{m=0}^{\infty} \sum_{m_{1}=0}^{m+1} i_{1}^{a} k_{1} l_{1}^{a} P_{i_{1}, k_{1}, l_{1}, m_{1}}\left(i-i_{1}\right)^{1-a}\left(l-l_{1}\right)^{1-a} \\
\quad \times\left(m+1-m_{1}\right) P_{i-i_{1}, k+1-k_{1}, l-l_{1}, m+1-m_{1}} \\
=\sum_{a=0}^{1}\left(\begin{array}{l}
1 \\
a
\end{array}\right) \sum_{i=1}^{\infty} \sum_{k=0}^{\infty} \sum_{l=0}^{\infty} \sum_{m=0}^{\infty} i^{a} k l^{a} P_{i, k, l, m} \sum_{i_{1}=1}^{\infty} \sum_{k_{1}=0}^{\infty} \sum_{l_{1}=0}^{\infty} i_{m_{1}=0}^{\infty-a} l_{1}^{1-a} m_{1} P_{i_{1} k_{1}, l_{1}, m_{1}} \\
=\sum_{a=0}^{1}\left(\begin{array}{l}
1 \\
a
\end{array}\right) \mu_{a, 1 a, 0} \mu_{1-a, 0,1-a, 1} \\
=\left(\frac{1 !}{0 ! 1 !} \mu_{0100} \mu_{1011}\right)+\left(\frac{1 !}{1 ! 0 !} \mu_{1110} \mu_{0001}\right) \\
=\mu_{1011} \mu_{0100}+\mu_{1110} \mu_{0001}
\end{aligned}
$$

Similarly,

$$
\begin{aligned}
\sum_{i=1}^{\infty} \sum_{i_{1}=1}^{i} \sum_{k=0}^{\infty} \sum_{k_{1}=0}^{k+1} \sum_{l=0}^{\infty} \sum_{l_{1}=0}^{l} \sum_{m=0}^{\infty} \sum_{m_{1}=0}^{m+1} i m_{1} P_{i_{1}, k_{1}, l_{1}, m_{1}}\left(k+1-k_{1}\right) P_{i-i_{1}, k+1-k_{1}, l-l_{1}, m+1-m_{1}} \\
=\sum_{i=1}^{\infty} \sum_{i_{1}=1}^{i} \sum_{k=0}^{\infty} \sum_{k_{1}=0}^{k+1} \sum_{l=0}^{\infty} \sum_{l_{1}=0}^{l} \sum_{m=0}^{\infty} \sum_{m_{1}=0}^{m+1}\left[i_{1}+\left(i-i_{1}\right)\right]\left[l_{1}+\left(l-l_{1}\right)\right] m_{1} P_{i_{1}, k_{1}, l_{1}, m_{1}} \\
\quad \times\left(k+1-k_{1}\right) P_{i-i_{1}, k+1-k_{1}, l-l_{1}, m+1-m_{1}} \\
=\sum_{a=0}^{1}\left(\begin{array}{l}
1 \\
a
\end{array}\right) \sum_{i=1}^{\infty} \sum_{i_{1}=1}^{i} \sum_{k=0}^{\infty} \sum_{k_{1}=0}^{k+1} \sum_{l=0}^{\infty} \sum_{l_{1}=0}^{l} \sum_{m=0}^{\infty} \sum_{m_{1}=0}^{m+1} i_{1}^{a} l_{1}^{a} m_{1} P_{i_{1}, k_{1}, l_{1}, m_{1}}\left(i-i_{1}\right)^{1-a}\left(l-l_{1}\right)^{1-a} \\
\quad \times\left(k+1-k_{1}\right) P_{i-i_{1}, k+1-k_{1}, l-l_{1}, m+1-m_{1}} \\
=\sum_{a=0}^{1}\left(\begin{array}{l}
1 \\
a
\end{array}\right) \sum_{i=1}^{\infty} \sum_{k=0}^{\infty} \sum_{l=0}^{\infty} \sum_{m=0}^{\infty} i^{a} l^{a} m P_{k, m} \sum_{i_{1}=1}^{\infty} \sum_{k_{1}=0}^{\infty} \sum_{l_{1}=0}^{\infty} \sum_{m_{1}=0}^{\infty} i_{1}^{1-a} k_{1} l_{1}^{1-a} P_{k_{1}, m_{1}} \\
=\sum_{a=0}^{1}\left(\begin{array}{l}
1 \\
a
\end{array}\right) \mu_{a, 0, a 1} \mu_{1-a, 1,1-a, 0} \\
=\left(\frac{1 !}{0 ! 1 !} \mu_{0001} \mu_{1110}\right)+\left(\frac{1 !}{1 ! 0 !} \mu_{1011} \mu_{0100}\right) \\
=\mu_{1011} \mu_{0100}+\mu_{1110} \mu_{0001}
\end{aligned}
$$




$$
\begin{aligned}
& \Rightarrow \frac{d \mu_{10010}}{d t}=-k_{p} C_{M} \sum_{i=1}^{\infty} \sum_{j=0}^{\infty} \sum_{k=0}^{\infty} \sum_{l=0}^{\infty} \sum_{m=0}^{\infty} i k l P_{i j k l m} \\
& +k_{p} C_{M} \sum_{i=1}^{\infty} \sum_{j=0}^{\infty} \sum_{k=0}^{\infty} \sum_{l=0}^{\infty} \sum_{m=0}^{\infty}(i+1) k l P_{i j k l m} \\
& -k_{p} C_{M} \sum_{i=1}^{\infty} \sum_{j=0}^{\infty} \sum_{k=0}^{\infty} \sum_{l=0}^{\infty} \sum_{m=0}^{\infty} i l m P_{i j k l m} \\
& +k_{p} C_{M} \sum_{i=1}^{\infty} \sum_{j=0}^{\infty} \sum_{k=0}^{\infty} \sum_{l=0}^{\infty} \sum_{m=0}^{\infty}(i+1) l m P_{i j k l m} \\
& -k_{t r t} C_{T} \sum_{i=1}^{\infty} \sum_{j=0}^{\infty} \sum_{k=0}^{\infty} \sum_{l=0}^{\infty} \sum_{m=0}^{\infty} i k l P_{i j k l m} \\
& +k_{t r t} C_{T} \sum_{i=1}^{\infty} \sum_{j=0}^{\infty} \sum_{k=0}^{\infty} \sum_{l=0}^{\infty} \sum_{m=0}^{\infty} i k(l+1) P_{i j k l m} \\
& -k_{t r t} \sum_{i=1}^{\infty} \sum_{j=0}^{\infty} \sum_{k=0}^{\infty} \sum_{l=0}^{\infty} \sum_{m=0}^{\infty} i k l P_{i j k l m} \sum_{i_{1}=1}^{\infty} \sum_{j_{1}=0}^{\infty} \sum_{k_{1}=0}^{\infty} \sum_{l_{1}=0}^{\infty} \sum_{m_{1}=0}^{\infty} l_{1} P_{i_{1} j_{1} k_{1} l_{1} m_{1}} \\
& -k_{t r t} \sum_{i=1}^{\infty} \sum_{j=0}^{\infty} \sum_{k=0}^{\infty} \sum_{l=0}^{\infty} \sum_{m=0}^{\infty} i l^{2} P_{i j k l m} \sum_{i_{1}=1}^{\infty} \sum_{j_{1}=0}^{\infty} \sum_{k_{1}=0}^{\infty} \sum_{l_{1}=0}^{\infty} \sum_{m_{1}=0}^{\infty} k_{1} P_{i_{1} j_{1} k_{1} l_{1} m_{1}} \\
& +k_{t r t} \sum_{i=1}^{\infty} \sum_{j=0}^{\infty} \sum_{k=0}^{\infty} \sum_{l=0}^{\infty} \sum_{m=0}^{\infty} i k(l+1) P_{i j k l m} \sum_{i_{1}=1}^{\infty} \sum_{j_{1}=0}^{\infty} \sum_{k_{1}=0}^{\infty} \sum_{l_{1}=0}^{\infty} \sum_{m_{1}=0}^{\infty} l_{1} P_{i_{1} j_{1} k_{1} l_{1} m_{1}} \\
& +k_{t r t} \sum_{i=1}^{\infty} \sum_{j=0}^{\infty} \sum_{k=0}^{\infty} \sum_{l=0}^{\infty} \sum_{m=}^{\infty} i(l-1) l P_{i j k l m} \sum_{i_{1}=1}^{\infty} \sum_{j_{1}=0}^{\infty} \sum_{k_{1}=0}^{\infty} \sum_{l_{1}=0}^{\infty} \sum_{m_{1}=0}^{\infty} k_{1} P_{i_{1} j_{1} k_{1} l_{1} m_{1}} \\
& -k_{t r p} \sum_{i=1}^{\infty} \sum_{j=0}^{\infty} \sum_{k=0}^{\infty} \sum_{l=0}^{\infty} \sum_{m=0}^{\infty} i k l P_{i j k l m} \sum_{i_{1}=1}^{\infty} \sum_{j_{1}=0}^{\infty} \sum_{k_{1}=0}^{\infty} \sum_{l_{1}=0}^{\infty} \sum_{m_{1}=0}^{\infty} i_{1} P_{i_{1} j_{1} k_{1} l_{1} m_{1}} \\
& -k_{\text {trp }} \sum_{i=1}^{\infty} \sum_{j=0}^{\infty} \sum_{k=0}^{\infty} \sum_{l=0}^{\infty} \sum_{m=0}^{\infty} i^{2} l P_{i j k l m} \sum_{i_{1}=1}^{\infty} \sum_{j_{1}=0}^{\infty} \sum_{k_{1}=0}^{\infty} \sum_{l_{1}=0}^{\infty} \sum_{m_{1}=0}^{\infty} k_{1} P_{i_{1} j_{1} k_{1} l_{1} m_{1}} \\
& +k_{t r p} \sum_{i=1}^{\infty} \sum_{j=0}^{\infty} \sum_{k=0}^{\infty} \sum_{l=0}^{\infty} \sum_{m=0}^{\infty} i k l P_{i j k l m} \sum_{i_{1}=1}^{\infty} \sum_{j_{1}=0}^{\infty} \sum_{k_{1}=0}^{\infty} \sum_{l_{1}=0}^{\infty} \sum_{m_{1}=0}^{\infty} i_{1} P_{i_{1} j_{1} k_{1} l_{1} m_{1}} \\
& +k_{t r p} \sum_{i=1}^{\infty} \sum_{j=0}^{\infty} \sum_{k=0}^{\infty} \sum_{l=0}^{\infty} \sum_{m=0}^{\infty} i^{2} l P_{i j k l m} \sum_{i_{1}=1}^{\infty} \sum_{j_{1}=0}^{\infty} \sum_{k_{1}=0}^{\infty} \sum_{l_{1}=0}^{\infty} \sum_{m_{1}=0}^{\infty} k_{1} P_{i_{1} j_{1} k_{1} l_{1} m_{1}}
\end{aligned}
$$




$$
\begin{aligned}
& -k_{\operatorname{trp}} \sum_{i=1}^{\infty} \sum_{j=0}^{\infty} \sum_{k=0}^{\infty} \sum_{l=0}^{\infty} \sum_{m=0}^{\infty} i \operatorname{lm} P_{i j k l m} \sum_{i_{1}=1}^{\infty} \sum_{j_{1}=0}^{\infty} \sum_{k_{1}=0}^{\infty} \sum_{l_{1}=0}^{\infty} \sum_{m_{1}=0}^{\infty} i_{1} P_{i_{1} j_{1} k_{1} l_{1} m_{1}} \\
& -k_{\operatorname{trp}} \sum_{i=1}^{\infty} \sum_{j=0}^{\infty} \sum_{k=0}^{\infty} \sum_{l=0}^{\infty} \sum_{m=0}^{\infty} i^{2} l P_{i j k l m} \sum_{i_{1}=1}^{\infty} \sum_{j_{1}=0}^{\infty} \sum_{k_{1}=0}^{\infty} \sum_{l_{1}=0}^{\infty} \sum_{m_{1}=0}^{\infty} m_{1} P_{i_{1} j_{1} k_{1} l_{1} m_{1}} \\
& +k_{\operatorname{trp}} \sum_{i=1}^{\infty} \sum_{j=0}^{\infty} \sum_{k=0}^{\infty} \sum_{l=0}^{\infty} \sum_{m=0}^{\infty} i l m P_{i j k l m} \sum_{i_{1}=1}^{\infty} \sum_{j_{1}=0}^{\infty} \sum_{k_{1}=0}^{\infty} \sum_{l_{1}=0}^{\infty} \sum_{m_{1}=0}^{\infty} i_{1} P_{i_{1} j_{1} k_{1} l_{1} m_{1}} \\
& +k_{\operatorname{trp}} \sum_{i=1}^{\infty} \sum_{j=0}^{\infty} \sum_{k=0}^{\infty} \sum_{l=0}^{\infty} \sum_{m=0}^{\infty} i^{2} l P_{i j k l m} \sum_{i_{1}=1}^{\infty} \sum_{j_{1}=0}^{\infty} \sum_{k_{1}=0}^{\infty} \sum_{l_{1}=0}^{\infty} \sum_{m_{1}=0}^{\infty} m_{1} P_{i_{1} j_{1} k_{1} l_{1} m_{1}} \\
& -k_{i n t r} \sum_{i=1}^{\infty} \sum_{j=0}^{\infty} \sum_{k=0}^{\infty} \sum_{l=0}^{\infty} \sum_{m=0}^{\infty} i k l P_{i j k l m} \\
& +k_{i n t r} \sum_{i=1}^{\infty} \sum_{j=0}^{\infty} \sum_{k=0}^{\infty} \sum_{l=0}^{\infty} \sum_{m=0}^{\infty} i k l P_{i j k l m} \\
& -k_{r} \sum_{i=1}^{\infty} \sum_{j=0}^{\infty} \sum_{k=0}^{\infty} \sum_{l=0}^{\infty} \sum_{m=0}^{\infty} i l m P_{i j k l m} \\
& +k_{r} \sum_{i=1}^{\infty} \sum_{j=0}^{\infty} \sum_{k=0}^{\infty} \sum_{l=0}^{\infty} \sum_{m=0}^{\infty} i \operatorname{lm} P_{i j k l m} \\
& -k_{t c} \sum_{i=1}^{\infty} \sum_{j=0}^{\infty} \sum_{k=0}^{\infty} \sum_{l=0}^{\infty} \sum_{m=0}^{\infty} i l k P_{i j k l m} \sum_{i_{1}=1}^{\infty} \sum_{j_{1}=0}^{\infty} \sum_{k_{1}=0}^{\infty} \sum_{l_{1}=0}^{\infty} \sum_{m_{1}=0}^{\infty} k_{1} P_{i_{1} j_{1} k_{1} l_{1} m_{1}} \\
& +\frac{1}{2}(2) k_{t c} \sum_{i=1}^{\infty} \sum_{j=0}^{\infty} \sum_{k=0}^{\infty} \sum_{l=0}^{\infty} \sum_{m=0}^{\infty} i l k P_{i j k l m} \sum_{i_{1}=1}^{\infty} \sum_{j_{1}=0}^{\infty} \sum_{k_{1}=0}^{\infty} \sum_{l_{1}=0}^{\infty} \sum_{m_{1}=0}^{\infty} k_{1} P_{i_{1} j_{1} k_{1} l_{1} m_{1}} \\
& -k_{t c} \sum_{i=1}^{\infty} \sum_{j=0}^{\infty} \sum_{k=0}^{\infty} \sum_{l=0}^{\infty} \sum_{m=0}^{\infty} i \operatorname{lm} P_{i j k l m} \sum_{i_{1}=1}^{\infty} \sum_{j_{1}=0}^{\infty} \sum_{k_{1}=0}^{\infty} \sum_{l_{1}=0}^{\infty} \sum_{m_{1}=0}^{\infty} m_{1} P_{i_{1} j_{1} k_{1} l_{1} m_{1}} \\
& +\frac{1}{2}(2) k_{t c} \sum_{i=1}^{\infty} \sum_{j=0}^{\infty} \sum_{k=0}^{\infty} \sum_{l=0}^{\infty} \sum_{m=0}^{\infty} i \operatorname{lm} P_{i j k l m} \sum_{i_{1}=1}^{\infty} \sum_{j_{1}=0}^{\infty} \sum_{k_{1}=0}^{\infty} \sum_{l_{1}=0}^{\infty} \sum_{m_{1}=0}^{\infty} m_{1} P_{i_{1} j_{1} k_{1} l_{1} m_{1}} \\
& -k_{t c} \sum_{i=1}^{\infty} \sum_{j=0}^{\infty} \sum_{k=0}^{\infty} \sum_{l=0}^{\infty} \sum_{m=0}^{\infty} i k l P_{i j k l m} \sum_{i_{1}=1}^{\infty} \sum_{j_{1}=0}^{\infty} \sum_{k_{1}=0}^{\infty} \sum_{l_{1}=0}^{\infty} \sum_{m_{1}=0}^{\infty} m_{1} P_{i_{1} j_{1} k_{1} l_{1} m_{1}} \\
& -k_{t c} \sum_{i=1}^{\infty} \sum_{j=0}^{\infty} \sum_{k=0}^{\infty} \sum_{l=0}^{\infty} \sum_{m=0}^{\infty} i \operatorname{lm} P_{i j k l m} \sum_{i_{1}=1}^{\infty} \sum_{j_{1}=0}^{\infty} \sum_{k_{1}=0}^{\infty} \sum_{l_{1}=0}^{\infty} \sum_{m_{1}=0}^{\infty} k_{1} P_{i_{1} j_{1} k_{1} l_{1} m_{1}} \\
& +\frac{1}{2}(2) k_{t c} \sum_{i=1}^{\infty} \sum_{j=0}^{\infty} \sum_{k=0}^{\infty} \sum_{l=0}^{\infty} \sum_{m=0}^{\infty} i k l P_{i j k l m} \sum_{i_{1}=1}^{\infty} \sum_{j_{1}=0}^{\infty} \sum_{k_{1}=0}^{\infty} \sum_{l_{1}=0}^{\infty} \sum_{m_{1}=0}^{\infty} m_{1} P_{i_{1} j_{1} k_{1} l_{1} m_{1}} \\
& +\frac{1}{2}(2) k_{t c} \sum_{i=1}^{\infty} \sum_{j=0}^{\infty} \sum_{k=0}^{\infty} \sum_{l=0}^{\infty} \sum_{m=0}^{\infty} i \operatorname{lm} P_{i j k l m} \sum_{i_{1}=1}^{\infty} \sum_{j_{1}=0}^{\infty} \sum_{k_{1}=0}^{\infty} \sum_{l_{1}=0}^{\infty} \sum_{m_{1}=0}^{\infty} k_{1} P_{i_{1} j_{1} k_{1} l_{1} m_{1}}
\end{aligned}
$$

using Equations (74), (98) and (99). 


$$
\begin{aligned}
& \Rightarrow \frac{d \mu_{10010}}{d t}=k_{p} C_{M} \sum_{i=1}^{\infty} \sum_{j=0}^{\infty} \sum_{k=0}^{\infty} \sum_{l=0}^{\infty} \sum_{m=0}^{\infty} k l P_{i j k l m} \\
& \quad+k_{p} C_{M} \sum_{i=1}^{\infty} \sum_{j=0}^{\infty} \sum_{k=0}^{\infty} \sum_{l=0}^{\infty} \sum_{m=0}^{\infty} l m P_{i j k l m} \\
& \quad+k_{t r t} C_{T} \sum_{i=1}^{\infty} \sum_{j=0}^{\infty} \sum_{k=0}^{\infty} \sum_{l=0}^{\infty} \sum_{m=0}^{\infty} i k P_{i j k l m} \\
& \quad+k_{t r t} \sum_{i=1}^{\infty} \sum_{j=0}^{\infty} \sum_{k=0}^{\infty} \sum_{l=0}^{\infty} \sum_{m=0}^{\infty} i k P_{i j k l m} \sum_{i_{1}=1}^{\infty} \sum_{j_{1}=0}^{\infty} \sum_{k_{1}=0}^{\infty} \sum_{l_{1}=0}^{\infty} \sum_{m_{1}=0}^{\infty} l_{1} P_{i_{1} j_{1} k_{1} l_{1} m_{1}} \\
& \quad-k_{t r t} \sum_{i=1}^{\infty} \sum_{j=0}^{\infty} \sum_{k=0}^{\infty} \sum_{l=0}^{\infty} \sum_{m=}^{\infty} i l P_{i j k l m} \sum_{i_{1}=1}^{\infty} \sum_{j_{1}=0}^{\infty} \sum_{k_{1}=0}^{\infty} \sum_{l_{1}=0}^{\infty} \sum_{m_{1}=0}^{\infty} k_{1} P_{i_{1} j_{1} k_{1} l_{1} m_{1}} \\
& \Rightarrow \frac{d \mu_{10010}}{d t}=k_{p} C_{M} \mu_{00110}+k_{p} C_{M} \mu_{00011}+k_{t r t} C_{T} \mu_{10100}+k_{t r t} \mu_{10100} \mu_{00010} \\
& -k_{t r t} \mu_{10010} \mu_{00100}
\end{aligned}
$$


3.3.7 Second moment w.r.t. the number of unrelaxed (just uncapped) radicals

$$
\begin{aligned}
& \frac{d \mu_{00002}}{d t}=\sum_{i=1}^{\infty} \sum_{j=0}^{\infty} \sum_{k=0}^{\infty} \sum_{l=0}^{\infty} \sum_{m=0}^{\infty} m^{2} \frac{d P_{i j k l m}}{d t} \\
& =-k_{p} C_{M} \sum_{i=1}^{\infty} \sum_{j=0}^{\infty} \sum_{k=0}^{\infty} \sum_{l=0}^{\infty} \sum_{m=0}^{\infty} k m^{2} P_{i j k l m} \\
& +k_{p} C_{M} \sum_{i=2}^{\infty} \sum_{j=0}^{\infty} \sum_{k=0}^{\infty} \sum_{l=0}^{\infty} \sum_{m=0}^{\infty} k m^{2} P_{i-1 j k l m} \\
& -k_{p} C_{M} \sum_{i=1}^{\infty} \sum_{j=0}^{\infty} \sum_{k=0}^{\infty} \sum_{l=0}^{\infty} \sum_{m=0}^{\infty} m^{3} P_{i j k l m} \\
& +k_{p} C_{M} \sum_{i=2}^{\infty} \sum_{j=0}^{\infty} \sum_{k=0}^{\infty} \sum_{l=0}^{\infty} \sum_{m=0}^{\infty} m^{3} P_{i-1 j k l m} \\
& -k_{t r t} C_{T} \sum_{i=1}^{\infty} \sum_{j=0}^{\infty} \sum_{k=0}^{\infty} \sum_{l=0}^{\infty} \sum_{m=0}^{\infty} k m^{2} P_{i j k l m} \\
& +k_{t r t} C_{T} \sum_{i=1}^{\infty} \sum_{j=0}^{\infty} \sum_{k=0}^{\infty} \sum_{l=1}^{\infty} \sum_{m=0}^{\infty}(k+1) m^{2} P_{i j k+1 l-1 m} \\
& -k_{t r t} \sum_{i=1}^{\infty} \sum_{j=0}^{\infty} \sum_{k=0}^{\infty} \sum_{l=0}^{\infty} \sum_{m=0}^{\infty} k m^{2} P_{i j k l m} \sum_{i_{1}=1}^{\infty} \sum_{j_{1}=0}^{\infty} \sum_{k_{1}=0}^{\infty} \sum_{l_{1}=0}^{\infty} \sum_{m_{1}=0}^{\infty} l_{1} P_{i_{1} j_{1} k_{1} l_{1} m_{1}} \\
& -k_{t r t} \sum_{i=1}^{\infty} \sum_{j=0}^{\infty} \sum_{k=0}^{\infty} \sum_{l=0}^{\infty} \sum_{m=0}^{\infty} l m^{2} P_{i j k l m} \sum_{i_{1}=1}^{\infty} \sum_{j_{1}=0}^{\infty} \sum_{k_{1}=0}^{\infty} \sum_{l_{1}=0}^{\infty} \sum_{m_{1}=0}^{\infty} k_{1} P_{i_{1} j_{1} k_{1} l_{1} m_{1}} \\
& +k_{t r t} \sum_{i=1}^{\infty} \sum_{j=0}^{\infty} \sum_{k=0}^{\infty} \sum_{l=1}^{\infty} \sum_{m=0}^{\infty}(k+1) m^{2} P_{i j k+1 l-1 m} \sum_{i_{1}=1}^{\infty} \sum_{j_{1}=0}^{\infty} \sum_{k_{1}=0}^{\infty} \sum_{l_{1}=0}^{\infty} \sum_{m_{1}=0}^{\infty} l_{1} P_{i_{1} j_{1} k_{1} l_{1} m_{1}} \\
& +k_{t r t} \sum_{i=1}^{\infty} \sum_{j=0}^{\infty} \sum_{k=0}^{\infty} \sum_{l=0}^{\infty} \sum_{m=1}^{\infty}(l+1) m^{2} P_{i j k l+1 m-1} \sum_{i_{1}=1}^{\infty} \sum_{j_{1}=0}^{\infty} \sum_{k_{1}=0}^{\infty} \sum_{l_{1}=0}^{\infty} \sum_{m_{1}=0}^{\infty} k_{1} P_{i_{1} j_{1} k_{1} l_{1} m_{1}} \\
& -k_{t r p} \sum_{i=1}^{\infty} \sum_{j=0}^{\infty} \sum_{k=0}^{\infty} \sum_{l=0}^{\infty} \sum_{m=0}^{\infty} k m^{2} P_{i j k l m} \sum_{i_{1}=1}^{\infty} \sum_{j_{1}=0}^{\infty} \sum_{k_{1}=0}^{\infty} \sum_{l_{1}=0}^{\infty} \sum_{m_{1}=0}^{\infty} i_{1} P_{i_{1} j_{1} k_{1} l_{1} m_{1}} \\
& -k_{\operatorname{trp}} \sum_{i=1}^{\infty} \sum_{j=0}^{\infty} \sum_{k=0}^{\infty} \sum_{l=0}^{\infty} \sum_{m=0}^{\infty} i m^{2} P_{i j k l m} \sum_{i_{1}=1}^{\infty} \sum_{j_{1}=0}^{\infty} \sum_{k_{1}=0}^{\infty} \sum_{l_{1}=0}^{\infty} \sum_{m_{1}=0}^{\infty} k_{1} P_{i_{1} j_{1} k_{1} l_{1} m_{1}} \\
& +k_{\operatorname{trp}} \sum_{i=1}^{\infty} \sum_{j=0}^{\infty} \sum_{k=0}^{\infty} \sum_{l=0}^{\infty} \sum_{m=0}^{\infty}(k+1) m^{2} P_{i j k+1 l m} \sum_{i_{1}=1}^{\infty} \sum_{j_{1}=0}^{\infty} \sum_{k_{1}=0}^{\infty} \sum_{l_{1}=0}^{\infty} \sum_{m_{1}=0}^{\infty} i_{1} P_{i_{1} j_{1} k_{1} l_{1} m_{1}} \\
& +k_{t r p} \sum_{i=1}^{\infty} \sum_{j=1}^{\infty} \sum_{k=1}^{\infty} \sum_{l=0}^{\infty} \sum_{m=0}^{\infty} i m^{2} P_{i j-1 k-1 l m} \sum_{i_{1}=1}^{\infty} \sum_{j_{1}=0}^{\infty} \sum_{k_{1}=0}^{\infty} \sum_{l_{1}=0}^{\infty} \sum_{m_{1}=0}^{\infty} k_{1} P_{i_{1} j_{1} k_{1} l_{1} m_{1}}
\end{aligned}
$$




$$
\begin{aligned}
& -k_{\operatorname{trp}} \sum_{i=1}^{\infty} \sum_{j=0}^{\infty} \sum_{k=0}^{\infty} \sum_{l=0}^{\infty} \sum_{m=0}^{\infty} m^{3} P_{i j k l m} \sum_{i_{1}=1}^{\infty} \sum_{j_{1}=0}^{\infty} \sum_{k_{1}=0}^{\infty} \sum_{l_{1}=0}^{\infty} \sum_{m_{1}=0}^{\infty} i_{1} P_{i_{1} j_{1} k_{1} l_{1} m_{1}} \\
& -k_{t r p} \sum_{i=1}^{\infty} \sum_{j=0}^{\infty} \sum_{k=0}^{\infty} \sum_{l=0}^{\infty} \sum_{m=0}^{\infty} i m^{2} P_{i j k l m} \sum_{i_{1}=1}^{\infty} \sum_{j_{1}=0}^{\infty} \sum_{k_{1}=0}^{\infty} \sum_{l_{1}=0}^{\infty} \sum_{m_{1}=0}^{\infty} m_{1} P_{i_{1} j_{1} k_{1} l_{1} m_{1}} \\
& +k_{\operatorname{trp}} \sum_{i=1}^{\infty} \sum_{j=0}^{\infty} \sum_{k=0}^{\infty} \sum_{l=0}^{\infty} \sum_{m=0}^{\infty} m^{2}(m+1) P_{i j k l m+1} \sum_{i_{1}=1}^{\infty} \sum_{j_{1}=0}^{\infty} \sum_{k_{1}=0}^{\infty} \sum_{l_{1}=0}^{\infty} \sum_{m_{1}=0}^{\infty} i_{1} P_{i_{1} j_{1} k_{1} l_{1} m_{1}} \\
& +k_{t r p} \sum_{i=1}^{\infty} \sum_{j=1}^{\infty} \sum_{k=0}^{\infty} \sum_{l=0}^{\infty} \sum_{m=1}^{\infty} i m^{2} P_{i j-1 k l m-1} \sum_{i_{1}=1}^{\infty} \sum_{j_{1}=0}^{\infty} \sum_{k_{1}=0}^{\infty} \sum_{l_{1}=0}^{\infty} \sum_{m_{1}=0}^{\infty} m_{1} P_{i_{1} j_{1} k_{1} l_{1} m_{1}} \\
& -k_{i n t r} \sum_{i=1}^{\infty} \sum_{j=0}^{\infty} \sum_{k=0}^{\infty} \sum_{l=0}^{\infty} \sum_{m=0}^{\infty} k m^{2} P_{i j k l m} \\
& +k_{i n t r} \sum_{i=1}^{\infty} \sum_{j=1}^{\infty} \sum_{k=0}^{\infty} \sum_{l=0}^{\infty} \sum_{m=0}^{\infty} k m^{2} P_{i j-1 k l m} \\
& -k_{r} \sum_{i=1}^{\infty} \sum_{j=0}^{\infty} \sum_{k=0}^{\infty} \sum_{l=0}^{\infty} \sum_{m=0}^{\infty} m^{3} P_{i j k l m} \\
& +k_{r} \sum_{i=1}^{\infty} \sum_{j=0}^{\infty} \sum_{k=1}^{\infty} \sum_{l=0}^{\infty} \sum_{m=0}^{\infty} m^{2}(m+1) P_{i j k-1 l m+1} \\
& -k_{t c} \sum_{i=1}^{\infty} \sum_{j=0}^{\infty} \sum_{k=0}^{\infty} \sum_{l=0}^{\infty} \sum_{m=0}^{\infty} k m^{2} P_{i j k l m} \sum_{i_{1}=1}^{\infty} \sum_{j_{1}=0}^{\infty} \sum_{k_{1}=0}^{\infty} \sum_{l_{1}=0}^{\infty} \sum_{m_{1}=0}^{\infty} k_{1} P_{i_{1} j_{1} k_{1} l_{1} m_{1}} \\
& +\frac{1}{2} k_{t c} \sum_{i=1}^{\infty} \sum_{j=0}^{\infty} \sum_{k=0}^{\infty} \sum_{l=0}^{\infty} \sum_{m=0}^{\infty} \sum_{i_{1}=1}^{i} \sum_{j_{1}=0}^{j} \sum_{k_{1}=0}^{k+2} \sum_{l_{1}=0}^{l} \sum_{m_{1}=0}^{m} m^{2}\left[k_{1} P_{i_{1} j_{1} k_{1} l_{1} m_{1}}\left(k+2-k_{1}\right) P_{i-i_{1}, j-j_{1}, k+2-k_{1}, l-l_{1} m-m_{1}}\right] \\
& -k_{t c} \sum_{i=1}^{\infty} \sum_{j=0}^{\infty} \sum_{k=0}^{\infty} \sum_{l=0}^{\infty} \sum_{m=0}^{\infty} m^{3} P_{i j k l m} \sum_{i_{1}=1}^{\infty} \sum_{j_{1}=0}^{\infty} \sum_{k_{1}=0}^{\infty} \sum_{l_{1}=0}^{\infty} \sum_{m_{1}=0}^{\infty} m_{1} P_{i_{1} j_{1} k_{1} l_{1} m_{1}} \\
& +\frac{1}{2} k_{t c} \sum_{i=1}^{\infty} \sum_{j=0}^{\infty} \sum_{k=0}^{\infty} \sum_{l=0}^{\infty} \sum_{m=0}^{\infty} \sum_{i_{1}=1}^{i} \sum_{j_{1}=0}^{j} \sum_{k_{1}=0}^{k} \sum_{l_{1}=0}^{l} \sum_{m_{1}=0}^{m+2}\left[m^{2} m_{1} P_{i_{1} j_{1} k_{1} l_{1} m_{1}}\left(m+2-m_{1}\right) P_{i-i_{1}, j-j_{1}, k-k_{1}, l-l_{1} m+2-m_{1}}\right] \\
& -k_{t c} \sum_{i=1}^{\infty} \sum_{j=0}^{\infty} \sum_{k=0}^{\infty} \sum_{l=0}^{\infty} \sum_{m=0}^{\infty} k m^{2} P_{i j k l m} \sum_{i_{1}=1}^{\infty} \sum_{j_{1}=0}^{\infty} \sum_{k_{1}=0}^{\infty} \sum_{l_{1}=0}^{\infty} \sum_{m_{1}=0}^{\infty} m_{1} P_{i_{1} j_{1} k_{1} l_{1} m_{1}} \\
& -k_{t c} \sum_{i=1}^{\infty} \sum_{j=0}^{\infty} \sum_{k=0}^{\infty} \sum_{l=0}^{\infty} \sum_{m=0}^{\infty} m^{3} P_{i j k l m} \sum_{i_{1}=1}^{\infty} \sum_{j_{1}=0}^{\infty} \sum_{k_{1}=0}^{\infty} \sum_{l_{1}=0}^{\infty} \sum_{m_{1}=0}^{\infty} k_{1} P_{i_{1} j_{1} k_{1} l_{1} m_{1}} \\
& +\frac{1}{2} k_{t c} \sum_{i=1}^{\infty} \sum_{j=0}^{\infty} \sum_{k=0}^{\infty} \sum_{l=0}^{\infty} \sum_{m=0}^{\infty} \sum_{i_{1}=1}^{i} \sum_{j_{1}=0}^{j} \sum_{k_{1}=0}^{k+1} \sum_{l_{1}=0}^{l} \sum_{m_{1}=0}^{m+1}\left[m^{2} k_{1} P_{i_{1} j_{1} k_{1} l_{1} m_{1}}\left(m+1-m_{1}\right) P_{\left.i-i_{1}, j-j_{1}, k+1-k_{1}, l-l_{1} m+1-m_{1}\right]}\right] \\
& +\frac{1}{2} k_{t c} \sum_{i=1}^{\infty} \sum_{j=0}^{\infty} \sum_{k=0}^{\infty} \sum_{l=0}^{\infty} \sum_{m=0}^{\infty} \sum_{i_{1}=1}^{i} \sum_{j_{1}=0}^{j} \sum_{k_{1}=0}^{k+1} \sum_{l_{1}=0}^{l} \sum_{m_{1}=0}^{m+1}\left[m^{2} m_{1} P_{i_{1} j_{1} k_{1} l_{1} m_{1}}\left(k+1-k_{1}\right) P_{i-i_{1}, j-j_{1}, k+1-k_{1}, l-l_{1} m+1-m_{1}}\right]
\end{aligned}
$$




$$
\begin{aligned}
& \Rightarrow \frac{d \mu_{00002}}{d t}=-k_{p} C_{M} \sum_{i=1}^{\infty} \sum_{j=0}^{\infty} \sum_{k=0}^{\infty} \sum_{l=0}^{\infty} \sum_{m=0}^{\infty} k m^{2} P_{i j k l m} \\
& +k_{p} C_{M} \sum_{i=1}^{\infty} \sum_{j=0}^{\infty} \sum_{k=0}^{\infty} \sum_{l=0}^{\infty} \sum_{m=0}^{\infty} k m^{2} P_{i j k l m} \\
& -k_{p} C_{M} \sum_{i=1}^{\infty} \sum_{j=0}^{\infty} \sum_{k=0}^{\infty} \sum_{l=0}^{\infty} \sum_{m=0}^{\infty} m^{3} P_{i j k l m} \\
& +k_{p} C_{M} \sum_{i=1}^{\infty} \sum_{j=0}^{\infty} \sum_{k=0}^{\infty} \sum_{l=0}^{\infty} \sum_{m=0}^{\infty} m^{3} P_{i j k l m} \\
& -k_{t r t} C_{T} \sum_{i=1}^{\infty} \sum_{j=0}^{\infty} \sum_{k=0}^{\infty} \sum_{l=0}^{\infty} \sum_{m=0}^{\infty} k m^{2} P_{i j k l m} \\
& +k_{t r t} C_{T} \sum_{i=1}^{\infty} \sum_{j=0}^{\infty} \sum_{k=0}^{\infty} \sum_{l=0}^{\infty} \sum_{m=0}^{\infty} k m^{2} P_{i j k l m} \\
& -k_{t r t} \sum_{i=1}^{\infty} \sum_{j=0}^{\infty} \sum_{k=0}^{\infty} \sum_{l=0}^{\infty} \sum_{m=0}^{\infty} k m^{2} P_{i j k l m} \sum_{i_{1}=1}^{\infty} \sum_{j_{1}=0}^{\infty} \sum_{k_{1}=0}^{\infty} \sum_{l_{1}=0}^{\infty} \sum_{m_{1}=0}^{\infty} l_{1} P_{i_{1} j_{1} k_{1} l_{1} m_{1}} \\
& \text { - } k_{t r t} \sum_{i=1}^{\infty} \sum_{j=0}^{\infty} \sum_{k=0}^{\infty} \sum_{l=0}^{\infty} \sum_{m=0}^{\infty} l m^{2} P_{i j k l m} \sum_{i_{1}=1}^{\infty} \sum_{j_{1}=0}^{\infty} \sum_{k_{1}=0}^{\infty} \sum_{l_{1}=0}^{\infty} \sum_{m_{1}=0}^{\infty} k_{1} P_{i_{1} j_{1} k_{1} l_{1} m_{1}} \\
& +k_{t r t} \sum_{i=1}^{\infty} \sum_{j=0}^{\infty} \sum_{k=0}^{\infty} \sum_{l=0}^{\infty} \sum_{m=0}^{\infty} k m^{2} P_{i j k l m} \sum_{i_{1}=1}^{\infty} \sum_{j_{1}=0}^{\infty} \sum_{k_{1}=0}^{\infty} \sum_{l_{1}=0}^{\infty} \sum_{m_{1}=0}^{\infty} l_{1} P_{i_{1} j_{1} k_{1} l_{1} m_{1}} \\
& +k_{t r t} \sum_{i=1}^{\infty} \sum_{j=0}^{\infty} \sum_{k=0}^{\infty} \sum_{l=0}^{\infty} \sum_{m=0}^{\infty} l(m+1)^{2} P_{i j k l m} \sum_{i_{1}=1}^{\infty} \sum_{j_{1}=0}^{\infty} \sum_{k_{1}=0}^{\infty} \sum_{l_{1}=0}^{\infty} \sum_{m_{1}=0}^{\infty} k_{1} P_{i_{1} j_{1} k_{1} l_{1} m_{1}} \\
& -k_{t r p} \sum_{i=1}^{\infty} \sum_{j=0}^{\infty} \sum_{k=0}^{\infty} \sum_{l=0}^{\infty} \sum_{m=0}^{\infty} k m^{2} P_{i j k l m} \sum_{i_{1}=1}^{\infty} \sum_{j_{1}=0}^{\infty} \sum_{k_{1}=0}^{\infty} \sum_{l_{1}=0}^{\infty} \sum_{m_{1}=0}^{\infty} i_{1} P_{i_{1} j_{1} k_{1} l_{1} m_{1}} \\
& -k_{t r p} \sum_{i=1}^{\infty} \sum_{j=0}^{\infty} \sum_{k=0}^{\infty} \sum_{l=0}^{\infty} \sum_{m=0}^{\infty} i m^{2} P_{i j k l m} \sum_{i_{1}=1}^{\infty} \sum_{j_{1}=0}^{\infty} \sum_{k_{1}=0}^{\infty} \sum_{l_{1}=0}^{\infty} \sum_{m_{1}=0}^{\infty} k_{1} P_{i_{1} j_{1} k_{1} l_{1} m_{1}} \\
& +k_{t r p} \sum_{i=1}^{\infty} \sum_{j=0}^{\infty} \sum_{k=0}^{\infty} \sum_{l=0}^{\infty} \sum_{m=0}^{\infty} k m^{2} P_{i j k l m} \sum_{i_{1}=1}^{\infty} \sum_{j_{1}=0}^{\infty} \sum_{k_{1}=0}^{\infty} \sum_{l_{1}=0}^{\infty} \sum_{m_{1}=0}^{\infty} i_{1} P_{i_{1} j_{1} k_{1} l_{1} m_{1}} \\
& +k_{t r p} \sum_{i=1}^{\infty} \sum_{j=0}^{\infty} \sum_{k=0}^{\infty} \sum_{l=0}^{\infty} \sum_{m=0}^{\infty} i m^{2} P_{i j k l m} \sum_{i_{1}=1}^{\infty} \sum_{j_{1}=0}^{\infty} \sum_{k_{1}=0}^{\infty} \sum_{l_{1}=0}^{\infty} \sum_{m_{1}=0}^{\infty} k_{1} P_{i_{1} j_{1} k_{1} l_{1} m_{1}}
\end{aligned}
$$




$$
\begin{aligned}
& -k_{t r p} \sum_{i=1}^{\infty} \sum_{j=0}^{\infty} \sum_{k=0}^{\infty} \sum_{l=0}^{\infty} \sum_{m=0}^{\infty} m^{3} P_{i j k l m} \sum_{i_{1}=1}^{\infty} \sum_{j_{1}=0}^{\infty} \sum_{k_{1}=0}^{\infty} \sum_{l_{1}=0}^{\infty} \sum_{m_{1}=0}^{\infty} i_{1} P_{i_{1} j_{1} k_{1} l_{1} m_{1}} \\
& -k_{t r p} \sum_{i=1}^{\infty} \sum_{j=0}^{\infty} \sum_{k=0}^{\infty} \sum_{l=0}^{\infty} \sum_{m=0}^{\infty} i m^{2} P_{i j k l m} \sum_{i_{1}=1}^{\infty} \sum_{j_{1}=0}^{\infty} \sum_{k_{1}=0}^{\infty} \sum_{l_{1}=0}^{\infty} \sum_{m_{1}=0}^{\infty} m_{1} P_{i_{1} j_{1} k_{1} l_{1} m_{1}} \\
& +k_{\text {trp }} \sum_{i=1}^{\infty} \sum_{j=0}^{\infty} \sum_{k=0}^{\infty} \sum_{l=0}^{\infty} \sum_{m=0}^{\infty}(m-1)^{2} m P_{i j k l m} \sum_{i_{1}=1}^{\infty} \sum_{j_{1}=0}^{\infty} \sum_{k_{1}=0}^{\infty} \sum_{l_{1}=0}^{\infty} \sum_{m_{1}=0}^{\infty} i_{1} P_{i_{1} j_{1} k_{1} l_{1} m_{1}} \\
& +k_{\text {trp }} \sum_{i=1}^{\infty} \sum_{j=0}^{\infty} \sum_{k=0}^{\infty} \sum_{l=0}^{\infty} \sum_{m=0}^{\infty} i(m+1)^{2} P_{i j k l m} \sum_{i_{1}=1}^{\infty} \sum_{j_{1}=0}^{\infty} \sum_{k_{1}=0}^{\infty} \sum_{l_{1}=0}^{\infty} \sum_{m_{1}=0}^{\infty} m_{1} P_{i_{1} j_{1} k_{1} l_{1} m_{1}} \\
& -k_{i n t r} \sum_{i=1}^{\infty} \sum_{j=0}^{\infty} \sum_{k=0}^{\infty} \sum_{l=0}^{\infty} \sum_{m=0}^{\infty} k m^{2} P_{i j k l m} \\
& +k_{i n t r} \sum_{i=1}^{\infty} \sum_{j=0}^{\infty} \sum_{k=0}^{\infty} \sum_{l=0}^{\infty} \sum_{m=0}^{\infty} k m^{2} P_{i j k l m} \\
& -k_{r} \sum_{i=1}^{\infty} \sum_{j=0}^{\infty} \sum_{k=0}^{\infty} \sum_{l=0}^{\infty} \sum_{m=0}^{\infty} m^{3} P_{i j k l m} \\
& +k_{r} \sum_{i=1}^{\infty} \sum_{j=0}^{\infty} \sum_{k=0}^{\infty} \sum_{l=0}^{\infty} \sum_{m=0}^{\infty}(m-1)^{2} m P_{i j k l m} \\
& -k_{t c} \sum_{i=1}^{\infty} \sum_{j=0}^{\infty} \sum_{k=0}^{\infty} \sum_{l=0}^{\infty} \sum_{m=0}^{\infty} k m^{2} P_{i j k l m} \sum_{i_{1}=1}^{\infty} \sum_{j_{1}=0}^{\infty} \sum_{k_{1}=0}^{\infty} \sum_{l_{1}=0}^{\infty} \sum_{m_{1}=0}^{\infty} k_{1} P_{i_{1} j_{1} k_{1} l_{1} m_{1}} \\
& +\frac{1}{2}(2) k_{t c} \sum_{i=1}^{\infty} \sum_{j=0}^{\infty} \sum_{k=0}^{\infty} \sum_{l=0}^{\infty} \sum_{m=0}^{\infty} k m^{2} P_{i j k l m} \sum_{i_{1}=1}^{\infty} \sum_{j_{1}=0}^{\infty} \sum_{k_{1}=0}^{\infty} \sum_{l_{1}=0}^{\infty} \sum_{m_{1}=0}^{\infty} k_{1} P_{i_{1} j_{1} k_{1} l_{1} m_{1}} \\
& -k_{t c} \sum_{i=1}^{\infty} \sum_{j=0}^{\infty} \sum_{k=0}^{\infty} \sum_{l=0}^{\infty} \sum_{m=0}^{\infty} m^{3} P_{i j k l m} \sum_{i_{1}=1}^{\infty} \sum_{j_{1}=0}^{\infty} \sum_{k_{1}=0}^{\infty} \sum_{l_{1}=0}^{\infty} \sum_{m_{1}=0}^{\infty} m_{1} P_{i_{1} j_{1} k_{1} l_{1} m_{1}} \\
& +\frac{1}{2}(2) k_{t c} \sum_{i=1}^{\infty} \sum_{j=0}^{\infty} \sum_{k=0}^{\infty} \sum_{l=0}^{\infty} \sum_{m=0}^{\infty} m^{3} P_{i j k l m} \sum_{i_{1}=1}^{\infty} \sum_{j_{1}=0}^{\infty} \sum_{k_{1}=0}^{\infty} \sum_{l_{1}=0}^{\infty} \sum_{m_{1}=0}^{\infty} m_{1} P_{i_{1} j_{1} k_{1} l_{1} m_{1}} \\
& +\frac{1}{2}(2) k_{t c} \sum_{i=1}^{\infty} \sum_{j=0}^{\infty} \sum_{k=0}^{\infty} \sum_{l=0}^{\infty} \sum_{m=0}^{\infty} m^{2} P_{i j k l m} \sum_{i_{1}=1}^{\infty} \sum_{j_{1}=0}^{\infty} \sum_{k_{1}=0}^{\infty} \sum_{l_{1}=0}^{\infty} \sum_{m_{1}=0}^{\infty} m_{1}^{2} P_{i_{1} j_{1} k_{1} l_{1} m_{1}} \\
& -\frac{1}{2}(8) k_{t c} \sum_{i=1}^{\infty} \sum_{j=0}^{\infty} \sum_{k=0}^{\infty} \sum_{l=0}^{\infty} \sum_{m=0}^{\infty} m P_{i j k l m} \sum_{i_{1}=1}^{\infty} \sum_{j_{1}=0}^{\infty} \sum_{k_{1}=0}^{\infty} \sum_{l_{1}=0}^{\infty} \sum_{m_{1}=0}^{\infty} m_{1}^{2} P_{i_{1} j_{1} k_{1} l_{1} m_{1}} \\
& +\frac{1}{2}(4) k_{t c} \sum_{i=1}^{\infty} \sum_{j=0}^{\infty} \sum_{k=0}^{\infty} \sum_{l=0}^{\infty} \sum_{m=0}^{\infty} m P_{i j k l m} \sum_{i_{1}=1}^{\infty} \sum_{j_{1}=0}^{\infty} \sum_{k_{1}=0}^{\infty} \sum_{l_{1}=0}^{\infty} \sum_{m_{1}=0}^{\infty} m_{1} P_{i_{1} j_{1} k_{1} l_{1} m_{1}}
\end{aligned}
$$




$$
\begin{aligned}
& -k_{t c} \sum_{i=1}^{\infty} \sum_{j=0}^{\infty} \sum_{k=0}^{\infty} \sum_{l=0}^{\infty} \sum_{m=0}^{\infty} k m^{2} P_{i j k l m} \sum_{i_{1}=1}^{\infty} \sum_{j_{1}=0}^{\infty} \sum_{k_{1}=0}^{\infty} \sum_{l_{1}=0}^{\infty} \sum_{m_{1}=0}^{\infty} m_{1} P_{i_{1} j_{1} k_{1} l_{1} m_{1}} \\
& -k_{t c} \sum_{i=1}^{\infty} \sum_{j=0}^{\infty} \sum_{k=0}^{\infty} \sum_{l=0}^{\infty} \sum_{m=0}^{\infty} m^{3} P_{i j k l m} \sum_{i_{1}=1}^{\infty} \sum_{j_{1}=0}^{\infty} \sum_{k_{1}=0}^{\infty} \sum_{l_{1}=0}^{\infty} \sum_{m_{1}=0}^{\infty} k_{1} P_{i_{1} j_{1} k_{1} l_{1} m_{1}} \\
& +\frac{1}{2}(2) k_{t c} \sum_{i=1}^{\infty} \sum_{j=0}^{\infty} \sum_{k=0}^{\infty} \sum_{l=0}^{\infty} \sum_{m=0}^{\infty} k m^{2} P_{i j k l m} \sum_{i_{1}=1}^{\infty} \sum_{j_{1}=0}^{\infty} \sum_{k_{1}=0}^{\infty} \sum_{l_{1}=0}^{\infty} \sum_{m_{1}=0}^{\infty} m_{1} P_{i_{1} j_{1} k_{1} l_{1} m_{1}} \\
& +\frac{1}{2}(2) k_{t c} \sum_{i=1}^{\infty} \sum_{j=0}^{\infty} \sum_{k=0}^{\infty} \sum_{l=0}^{\infty} \sum_{m=0}^{\infty} m^{3} P_{i j k l m} \sum_{i_{1}=1}^{\infty} \sum_{j_{1}=0}^{\infty} \sum_{k_{1}=0}^{\infty} \sum_{l_{1}=0}^{\infty} \sum_{m_{1}=0}^{\infty} k_{1} P_{i_{1} j_{1} k_{1} l_{1} m_{1}} \\
& +\frac{1}{2}(4) k_{t c} \sum_{i=1}^{\infty} \sum_{j=0}^{\infty} \sum_{k=0}^{\infty} \sum_{l=0}^{\infty} \sum_{m=0}^{\infty} m^{2} P_{i j k l m} \sum_{i_{1}=1}^{\infty} \sum_{j_{1}=0}^{\infty} \sum_{k_{1}=0}^{\infty} \sum_{l_{1}=0}^{\infty} \sum_{m_{1}=0}^{\infty} k_{1} m_{1} P_{i_{1} j_{1} k_{1} l_{1} m_{1}} \\
& -\frac{1}{2}(4) k_{t c} \sum_{i=1}^{\infty} \sum_{j=0}^{\infty} \sum_{k=0}^{\infty} \sum_{l=0}^{\infty} \sum_{m=0}^{\infty} m^{2} P_{i j k l m} \sum_{i_{1}=1}^{\infty} \sum_{j_{1}=0}^{\infty} \sum_{k_{1}=0}^{\infty} \sum_{l_{1}=0}^{\infty} \sum_{m_{1}=0}^{\infty} k_{1} P_{i_{1} j_{1} k_{1} l_{1} m_{1}} \\
& -\frac{1}{2}(4) k_{t c} \sum_{i=1}^{\infty} \sum_{j=0}^{\infty} \sum_{k=0}^{\infty} \sum_{l=0}^{\infty} \sum_{m=0}^{\infty} k_{m} P_{i j k l m} \sum_{i_{1}=1}^{\infty} \sum_{j_{1}=0}^{\infty} \sum_{k_{1}=0}^{\infty} \sum_{l_{1}=0}^{\infty} \sum_{m_{1}=0}^{\infty} m_{1} P_{i_{1} j_{1} k_{1} l_{1} m_{1}} \\
& +\frac{1}{2}(2) k_{t c} \sum_{i=1}^{\infty} \sum_{j=0}^{\infty} \sum_{k=0}^{\infty} \sum_{l=0}^{\infty} \sum_{m=0}^{\infty} m P_{i j k l m} \sum_{i_{1}=1}^{\infty} \sum_{j_{1}=0}^{\infty} \sum_{k_{1}=0}^{\infty} \sum_{l_{1}=0}^{\infty} \sum_{m_{1}=0}^{\infty} k_{1} P_{i_{1} j_{1} k_{1} l_{1} m_{1}}
\end{aligned}
$$

using Equations (67), (81), (82) and (83).

$$
\begin{aligned}
& \Rightarrow \frac{d \mu_{00002}}{d t}=k_{\text {trt }} \sum_{i=1}^{\infty} \sum_{j=0}^{\infty} \sum_{k=0}^{\infty} \sum_{l=0}^{\infty} \sum_{m=0}^{\infty}(2 l m+l) P_{i j k l m} \sum_{i_{1}=1}^{\infty} \sum_{j_{1}=0}^{\infty} \sum_{k_{1}=0}^{\infty} \sum_{l_{1}=0}^{\infty} \sum_{m_{1}=0}^{\infty} k_{1} P_{i_{1} j_{1} k_{1} l_{1} m_{1}} \\
& \quad+k_{r} \sum_{i=1}^{\infty} \sum_{j=0}^{\infty} \sum_{k=0}^{\infty} \sum_{l=0}^{\infty} \sum_{m=0}^{\infty}\left(m-2 m^{2}\right) P_{i j k l m} \\
& \quad+k_{t r p} \sum_{i=1}^{\infty} \sum_{j=0}^{\infty} \sum_{k=0}^{\infty} \sum_{l=0}^{\infty} \sum_{m=0}^{\infty}\left(m-2 m^{2}\right) P_{i j k l m} \sum_{i_{1}=1}^{\infty} \sum_{j_{1}=0}^{\infty} \sum_{k_{1}=0}^{\infty} \sum_{l_{1}=0}^{\infty} \sum_{m_{1}=0}^{\infty} i_{1} P_{i_{1} j_{1} k_{1} l_{1} m_{1}} \\
& \quad+k_{t r p} \sum_{i=1}^{\infty} \sum_{j=0}^{\infty} \sum_{k=0}^{\infty} \sum_{l=0}^{\infty} \sum_{m=0}^{\infty}(2 i m+i) P_{i j k l m} \sum_{i_{1}=1}^{\infty} \sum_{j_{1}=0}^{\infty} \sum_{k_{1}=0}^{\infty} \sum_{l_{1}=0}^{\infty} \sum_{m_{1}=0}^{\infty} m_{1} P_{i_{1} j_{1} k_{1} l_{1} m_{1}} \\
& \quad+k_{t c} \sum_{i=1}^{\infty} \sum_{j=0}^{\infty} \sum_{k=0}^{\infty} \sum_{l=0}^{\infty} \sum_{m=0}^{\infty} m^{2} P_{i j k l m} \sum_{i_{1}=1}^{\infty} \sum_{j_{1}=0}^{\infty} \sum_{k_{1}=0}^{\infty} \sum_{l_{1}=0}^{\infty} \sum_{m_{1}=0}^{\infty} m_{1}^{2} P_{i_{1}} j_{1} k_{1} l_{1} m_{1} \\
& \quad-4 k_{t c} \sum_{i=1}^{\infty} \sum_{j=0}^{\infty} \sum_{k=0}^{\infty} \sum_{l=0}^{\infty} \sum_{m=0}^{\infty} m P_{i j k l m} \sum_{i_{1}=1}^{\infty} \sum_{j_{1}=0}^{\infty} \sum_{k_{1}=0}^{\infty} \sum_{l_{1}=0}^{\infty} \sum_{m_{1}=0}^{\infty} m_{1}^{2} P_{i_{1}} j_{1} k_{1} l_{1} m_{1} \\
& +2 k_{t c} \sum_{i=1}^{\infty} \sum_{j=0}^{\infty} \sum_{k=0}^{\infty} \sum_{l=0}^{\infty} \sum_{m=0}^{\infty} m P_{i j k l m} \sum_{i_{1}=1}^{\infty} \sum_{j_{1}=0}^{\infty} \sum_{k_{1}=0}^{\infty} \sum_{l_{1}=0}^{\infty} \sum_{m_{1}=0}^{\infty} m_{1} P_{i_{1}} j_{1} k_{1} l_{1} m_{1}
\end{aligned}
$$




$$
\begin{aligned}
& +2 k_{t c} \sum_{i=1}^{\infty} \sum_{j=0}^{\infty} \sum_{k=0}^{\infty} \sum_{l=0}^{\infty} \sum_{m=0}^{\infty} m^{2} P_{i j k l m} \sum_{i_{1}=1}^{\infty} \sum_{j_{1}=0}^{\infty} \sum_{k_{1}=0}^{\infty} \sum_{l_{1}=0}^{\infty} \sum_{m_{1}=0}^{\infty} k_{1} m_{1} P_{i_{1} j_{1} k_{1} l_{1} m_{1}} \\
& -2 k_{t c} \sum_{i=1}^{\infty} \sum_{j=0}^{\infty} \sum_{k=0}^{\infty} \sum_{l=0}^{\infty} \sum_{m=0}^{\infty} m^{2} P_{i j k l m} \sum_{i_{1}=1}^{\infty} \sum_{j_{1}=0}^{\infty} \sum_{k_{1}=0}^{\infty} \sum_{l_{1}=0}^{\infty} \sum_{m_{1}=0}^{\infty} k_{1} P_{i_{1} j_{1} k_{1} l_{1} m_{1}} \\
& \quad-2 k_{t c} \sum_{i=1}^{\infty} \sum_{j=0}^{\infty} \sum_{k=0}^{\infty} \sum_{l=0}^{\infty} \sum_{m=0}^{\infty} k m P_{i j k l m} \sum_{i_{1}=1}^{\infty} \sum_{j_{1}=0}^{\infty} \sum_{k_{1}=0}^{\infty} \sum_{l_{1}=0}^{\infty} \sum_{m_{1}=0}^{\infty} m_{1} P_{i_{1} j_{1} k_{1} l_{1} m_{1}} \\
& \quad+k_{t c} \sum_{i=1}^{\infty} \sum_{j=0}^{\infty} \sum_{k=0}^{\infty} \sum_{l=0}^{\infty} \sum_{m=0}^{\infty} m P_{i j k l m} \sum_{i_{1}=1}^{\infty} \sum_{j_{1}=0}^{\infty} \sum_{k_{1}=0}^{\infty} \sum_{l_{1}=0}^{\infty} \sum_{m_{1}=0}^{\infty} k_{1} P_{i_{1} j_{1} k_{1} l_{1} m_{1}} \\
& \Rightarrow \frac{d \mu_{00002}}{d t}=k_{t r t} \mu_{00100}\left(2 \mu_{00011}+\mu_{00010}\right)+k_{t r p}\left(\mu_{00001}-2 \mu_{00002}\right) \mu_{10000} \\
& +k_{t r p}\left(2 \mu_{10001}+\mu_{10000}\right) \mu_{00001}-k_{r}\left(2 \mu_{00002}-\mu_{00001}\right) \\
& +k_{t c}\left[\left(\mu_{00002}\right)^{2}-4 \mu_{00002} \mu_{00001}+2\left(\mu_{00001}\right)^{2}\right] \\
& +2 k_{t c} \mu_{00002}\left(\mu_{00101}-\mu_{00100}\right)+k_{t c} \mu_{00001}\left(\mu_{00100}-2 \mu_{00101}\right)
\end{aligned}
$$


3.3.8 Second moment w.r.t. the number of active (uncapped) radicals and inactive (capped) radicals

$$
\begin{aligned}
& \frac{d \mu_{00110}}{d t}=\sum_{i=1}^{\infty} \sum_{j=0}^{\infty} \sum_{k=0}^{\infty} \sum_{l=0}^{\infty} \sum_{m=0}^{\infty} k l \frac{d P_{i j k l m}}{d t} \\
& =-k_{p} C_{M} \sum_{i=1}^{\infty} \sum_{j=0}^{\infty} \sum_{k=0}^{\infty} \sum_{l=0}^{\infty} \sum_{m=0}^{\infty} k^{2} l P_{i j k l m} \\
& +k_{p} C_{M} \sum_{i=2}^{\infty} \sum_{j=0}^{\infty} \sum_{k=0}^{\infty} \sum_{l=0}^{\infty} \sum_{m=0}^{\infty} k^{2} l P_{i-1 j k l m} \\
& -k_{p} C_{M} \sum_{i=1}^{\infty} \sum_{j=0}^{\infty} \sum_{k=0}^{\infty} \sum_{l=0}^{\infty} \sum_{m=0}^{\infty} k l m P_{i j k l m} \\
& +k_{p} C_{M} \sum_{i=2}^{\infty} \sum_{j=0}^{\infty} \sum_{k=0}^{\infty} \sum_{l=0}^{\infty} \sum_{m=0}^{\infty} k l m P_{i-1 j k l m} \\
& -k_{t r t} C_{T} \sum_{i=1}^{\infty} \sum_{j=0}^{\infty} \sum_{k=0}^{\infty} \sum_{l=0}^{\infty} \sum_{m=0}^{\infty} k^{2} l P_{i j k l m} \\
& +k_{t r t} C_{T} \sum_{i=1}^{\infty} \sum_{j=0}^{\infty} \sum_{k=0}^{\infty} \sum_{l=1}^{\infty} \sum_{m=0}^{\infty} k(k+1) l P_{i j k+1 l-1 m} \\
& -k_{t r t} \sum_{i=1}^{\infty} \sum_{j=0}^{\infty} \sum_{k=0}^{\infty} \sum_{l=0}^{\infty} \sum_{m=0}^{\infty} k^{2} l P_{i j k l m} \sum_{i_{1}=1}^{\infty} \sum_{j_{1}=0}^{\infty} \sum_{k_{1}=0}^{\infty} \sum_{l_{1}=0}^{\infty} \sum_{m_{1}=0}^{\infty} l_{1} P_{i_{1} j_{1} k_{1} l_{1} m_{1}} \\
& \text { - } k_{t r t} \sum_{i=1}^{\infty} \sum_{j=0}^{\infty} \sum_{k=0}^{\infty} \sum_{l=0}^{\infty} \sum_{m=0}^{\infty} k l^{2} P_{i j k l m} \sum_{i_{1}=1}^{\infty} \sum_{j_{1}=0}^{\infty} \sum_{k_{1}=0}^{\infty} \sum_{l_{1}=0}^{\infty} \sum_{m_{1}=0}^{\infty} k_{1} P_{i_{1} j_{1} k_{1} l_{1} m_{1}} \\
& +k_{t r t} \sum_{i=1}^{\infty} \sum_{j=0}^{\infty} \sum_{k=0}^{\infty} \sum_{l=1}^{\infty} \sum_{m=0}^{\infty} k(k+1) l P_{i j k+1 l-1 m} \sum_{i_{1}=1}^{\infty} \sum_{j_{1}=0}^{\infty} \sum_{k_{1}=0}^{\infty} \sum_{l_{1}=0}^{\infty} \sum_{m_{1}=0}^{\infty} l_{1} P_{i_{1} j_{1}} k_{1} l_{1} m_{1} \\
& +k_{t r t} \sum_{i=1}^{\infty} \sum_{j=0}^{\infty} \sum_{k=0}^{\infty} \sum_{l=0}^{\infty} \sum_{m=1}^{\infty} k l(l+1) P_{i j k l+1 m-1} \sum_{i_{1}=1}^{\infty} \sum_{j_{1}=0}^{\infty} \sum_{k_{1}=0}^{\infty} \sum_{l_{1}=0}^{\infty} \sum_{m_{1}=0}^{\infty} k_{1} P_{i_{1} j_{1} k_{1} l_{1} m_{1}} \\
& -k_{\text {trp }} \sum_{i=1}^{\infty} \sum_{j=0}^{\infty} \sum_{k=0}^{\infty} \sum_{l=0}^{\infty} \sum_{m=0}^{\infty} k^{2} l P_{i j k l m} \sum_{i_{1}=1}^{\infty} \sum_{j_{1}=0}^{\infty} \sum_{k_{1}=0}^{\infty} \sum_{l_{1}=0}^{\infty} \sum_{m_{1}=0}^{\infty} i_{1} P_{i_{1} j_{1} k_{1} l_{1} m_{1}} \\
& -k_{t r p} \sum_{i=1}^{\infty} \sum_{j=0}^{\infty} \sum_{k=0}^{\infty} \sum_{l=0}^{\infty} \sum_{m=0}^{\infty} i k l P_{i j k l m} \sum_{i_{1}=1}^{\infty} \sum_{j_{1}=0}^{\infty} \sum_{k_{1}=0}^{\infty} \sum_{l_{1}=0}^{\infty} \sum_{m_{1}=0}^{\infty} k_{1} P_{i_{1} j_{1} k_{1} l_{1} m_{1}} \\
& +k_{t r p} \sum_{i=1}^{\infty} \sum_{j=0}^{\infty} \sum_{k=0}^{\infty} \sum_{l=0}^{\infty} \sum_{m=0}^{\infty} k(k+1) l P_{i j k+1 l m} \sum_{i_{1}=1}^{\infty} \sum_{j_{1}=0}^{\infty} \sum_{k_{1}=0}^{\infty} \sum_{l_{1}=0}^{\infty} \sum_{m_{1}=0}^{\infty} i_{1} P_{i_{1} j_{1} k_{1} l_{1} m_{1}} \\
& +k_{t r p} \sum_{i=1}^{\infty} \sum_{j=1}^{\infty} \sum_{k=1}^{\infty} \sum_{l=0}^{\infty} \sum_{m=0}^{\infty} i k l P_{i j-1 k-1 l m} \sum_{i_{1}=1}^{\infty} \sum_{j_{1}=0}^{\infty} \sum_{k_{1}=0}^{\infty} \sum_{l_{1}=0}^{\infty} \sum_{m_{1}=0}^{\infty} k_{1} P_{i_{1} j_{1} k_{1} l_{1} m_{1}}
\end{aligned}
$$




$$
\begin{aligned}
& -k_{t r p} \sum_{i=1}^{\infty} \sum_{j=0}^{\infty} \sum_{k=0}^{\infty} \sum_{l=0}^{\infty} \sum_{m=0}^{\infty} k l m P_{i j k l m} \sum_{i_{1}=1}^{\infty} \sum_{j_{1}=0}^{\infty} \sum_{k_{1}=0}^{\infty} \sum_{l_{1}=0}^{\infty} \sum_{m_{1}=0}^{\infty} i_{1} P_{i_{1} j_{1} k_{1} l_{1} m_{1}} \\
& -k_{t r p} \sum_{i=1}^{\infty} \sum_{j=0}^{\infty} \sum_{k=0}^{\infty} \sum_{l=0}^{\infty} \sum_{m=0}^{\infty} i k l P_{i j k l m} \sum_{i_{1}=1}^{\infty} \sum_{j_{1}=0}^{\infty} \sum_{k_{1}=0}^{\infty} \sum_{l_{1}=0}^{\infty} \sum_{m_{1}=0}^{\infty} m_{1} P_{i_{1} j_{1} k_{1} l_{1} m_{1}} \\
& +k_{\operatorname{trp}} \sum_{i=1}^{\infty} \sum_{j=0}^{\infty} \sum_{k=0}^{\infty} \sum_{l=0}^{\infty} \sum_{m=0}^{\infty} k l(m+1) P_{i j k l m+1} \sum_{i_{1}=1}^{\infty} \sum_{j_{1}=0}^{\infty} \sum_{k_{1}=0}^{\infty} \sum_{l_{1}=0}^{\infty} \sum_{m_{1}=0}^{\infty} i_{1} P_{i_{1} j_{1} k_{1} l_{1} m_{1}} \\
& +k_{t r p} \sum_{i=1}^{\infty} \sum_{j=1}^{\infty} \sum_{k=0}^{\infty} \sum_{l=0}^{\infty} \sum_{m=1}^{\infty} i k l P_{i j-1 k l m-1} \sum_{i_{1}=1}^{\infty} \sum_{j_{1}=0}^{\infty} \sum_{k_{1}=0}^{\infty} \sum_{l_{1}=0}^{\infty} \sum_{m_{1}=0}^{\infty} m_{1} P_{i_{1} j_{1} k_{1} l_{1} m_{1}} \\
& -k_{\text {intr }} \sum_{i=1}^{\infty} \sum_{j=0}^{\infty} \sum_{k=0}^{\infty} \sum_{l=0}^{\infty} \sum_{m=0}^{\infty} k^{2} l P_{i j k l m} \\
& +k_{i n t r} \sum_{i=1}^{\infty} \sum_{j=1}^{\infty} \sum_{k=0}^{\infty} \sum_{l=0}^{\infty} \sum_{m=0}^{\infty} k^{2} l P_{i j-1 k l m} \\
& -k_{r} \sum_{i=1}^{\infty} \sum_{j=0}^{\infty} \sum_{k=0}^{\infty} \sum_{l=0}^{\infty} \sum_{m=0}^{\infty} k l m P_{i j k l m} \\
& +k_{r} \sum_{i=1}^{\infty} \sum_{j=0}^{\infty} \sum_{k=1}^{\infty} \sum_{l=0}^{\infty} \sum_{m=0}^{\infty} k l(m+1) P_{i j k-1 l m+1} \\
& -k_{t c} \sum_{i=1}^{\infty} \sum_{j=0}^{\infty} \sum_{k=0}^{\infty} \sum_{l=0}^{\infty} \sum_{m=0}^{\infty} k^{2} l P_{i j k l m} \sum_{i_{1}=1}^{\infty} \sum_{j_{1}=0}^{\infty} \sum_{k_{1}=0}^{\infty} \sum_{l_{1}=0}^{\infty} \sum_{m_{1}=0}^{\infty} k_{1} P_{i_{1} j_{1} k_{1} l_{1} m_{1}} \\
& +\frac{1}{2} k_{t c} \sum_{i=1}^{\infty} \sum_{j=0}^{\infty} \sum_{k=0}^{\infty} \sum_{l=0}^{\infty} \sum_{m=0}^{\infty} \sum_{i_{1}=1}^{i} \sum_{j_{1}=0}^{j} \sum_{k_{1}=0}^{k+2} \sum_{l_{1}=0}^{l} \sum_{m_{1}=0}^{m} k l\left[k_{1} P_{i_{1} j_{1} k_{1} l_{1} m_{1}}\left(k-k_{1}+2\right) P_{i-i_{1}, j-j_{1}, k-k_{1}+2, l-l_{1} m-m_{1}}\right] \\
& -k_{t c} \sum_{i=1}^{\infty} \sum_{j=0}^{\infty} \sum_{k=0}^{\infty} \sum_{l=0}^{\infty} \sum_{m=0}^{\infty} k l m P_{i j k l m} \sum_{i_{1}=1}^{\infty} \sum_{j_{1}=0}^{\infty} \sum_{k_{1}=0}^{\infty} \sum_{l_{1}=0}^{\infty} \sum_{m_{1}=0}^{\infty} m_{1} P_{i_{1} j_{1} k_{1} l_{1} m_{1}} \\
& +\frac{1}{2} k_{t c} \sum_{i=1}^{\infty} \sum_{j=0}^{\infty} \sum_{k=0}^{\infty} \sum_{l=0}^{\infty} \sum_{m=0}^{\infty} \sum_{i_{1}=1}^{i} \sum_{j_{1}=0}^{j} \sum_{k_{1}=0}^{k} \sum_{l_{1}=0}^{l} \sum_{m_{1}=0}^{m+2}\left[k l m_{1} P_{i_{1} j_{1} k_{1} l_{1} m_{1}}\left(m+2-m_{1}\right) P_{i-i_{1}, j-j_{1}, k-k_{1}, l-l_{1} m+2-m_{1}}\right] \\
& -k_{t c} \sum_{i=1}^{\infty} \sum_{j=0}^{\infty} \sum_{k=0}^{\infty} \sum_{l=0}^{\infty} \sum_{m=0}^{\infty} k^{2} l P_{i j k l m} \sum_{i_{1}=1}^{\infty} \sum_{j_{1}=0}^{\infty} \sum_{k_{1}=0}^{\infty} \sum_{l_{1}=0}^{\infty} \sum_{m_{1}=0}^{\infty} m_{1} P_{i_{1} j_{1} k_{1} l_{1} m_{1}} \\
& -k_{t c} \sum_{i=1}^{\infty} \sum_{j=0}^{\infty} \sum_{k=0}^{\infty} \sum_{l=0}^{\infty} \sum_{m=0}^{\infty} k \operatorname{lm} P_{i j k l m} \sum_{i_{1}=1}^{\infty} \sum_{j_{1}=0}^{\infty} \sum_{k_{1}=0}^{\infty} \sum_{l_{1}=0}^{\infty} \sum_{m_{1}=0}^{\infty} k_{1} P_{i_{1} j_{1} k_{1} l_{1} m_{1}} \\
& +\frac{1}{2} k_{t c} \sum_{i=1}^{\infty} \sum_{j=0}^{\infty} \sum_{k=0}^{\infty} \sum_{l=0}^{\infty} \sum_{m=0}^{\infty} \sum_{i_{1}=1}^{i} \sum_{j_{1}=0}^{j} \sum_{k_{1}=0}^{k+1} \sum_{l_{1}=0}^{l} \sum_{m_{1}=0}^{m+1}\left[k l k_{1} P_{i_{1} j_{1} k_{1} l_{1} m_{1}}\left(m+1-m_{1}\right) P_{\left.i-i_{1}, j-j_{1}, k+1-k_{1}, l-l_{1} m+1-m_{1}\right]}\right] \\
& +\frac{1}{2} k_{t c} \sum_{i=1}^{\infty} \sum_{j=0}^{\infty} \sum_{k=0}^{\infty} \sum_{l=0}^{\infty} \sum_{m=0}^{\infty} \sum_{i_{1}=1}^{i} \sum_{j_{1}=0}^{j} \sum_{k_{1}=0}^{k+1} \sum_{l_{1}=0}^{l} \sum_{m_{1}=0}^{m+1}\left[k l m_{1} P_{i_{1} j_{1} k_{1} l_{1} m_{1}}\left(k+1-k_{1}\right) P_{\left.i-i_{1}, j-j_{1}, k+1-k_{1}, l-l_{1} m+1-m_{1}\right]}\right]
\end{aligned}
$$




$$
\begin{aligned}
& \Rightarrow \frac{d \mu_{00110}}{d t}=-k_{p} C_{M} \sum_{i=1}^{\infty} \sum_{j=0}^{\infty} \sum_{k=0}^{\infty} \sum_{l=0}^{\infty} \sum_{m=0}^{\infty} k^{2} l P_{i j k l m} \\
& +k_{p} C_{M} \sum_{i=1}^{\infty} \sum_{j=0}^{\infty} \sum_{k=0}^{\infty} \sum_{l=0}^{\infty} \sum_{m=0}^{\infty} k^{2} l P_{i j k l m} \\
& -k_{p} C_{M} \sum_{i=1}^{\infty} \sum_{j=0}^{\infty} \sum_{k=0}^{\infty} \sum_{l=0}^{\infty} \sum_{m=0}^{\infty} k l m P_{i j k l m} \\
& +k_{p} C_{M} \sum_{i=1}^{\infty} \sum_{j=0}^{\infty} \sum_{k=0}^{\infty} \sum_{l=0}^{\infty} \sum_{m=0}^{\infty} k l m P_{i j k l m} \\
& -k_{t r t} C_{T} \sum_{i=1}^{\infty} \sum_{j=0}^{\infty} \sum_{k=0}^{\infty} \sum_{l=0}^{\infty} \sum_{m=0}^{\infty} k^{2} l P_{i j k l m} \\
& +k_{t r t} C_{T} \sum_{i=1}^{\infty} \sum_{j=0}^{\infty} \sum_{k=0}^{\infty} \sum_{l=0}^{\infty} \sum_{m=0}^{\infty}(k-1) k(l+1) P_{i j k l m} \\
& -k_{t r t} \sum_{i=1}^{\infty} \sum_{j=0}^{\infty} \sum_{k=0}^{\infty} \sum_{l=0}^{\infty} \sum_{m=0}^{\infty} k^{2} l P_{i j k l m} \sum_{i_{1}=1}^{\infty} \sum_{j_{1}=0}^{\infty} \sum_{k_{1}=0}^{\infty} \sum_{l_{1}=0}^{\infty} \sum_{m_{1}=0}^{\infty} l_{1} P_{i_{1} j_{1} k_{1} l_{1} m_{1}} \\
& -k_{t r t} \sum_{i=1}^{\infty} \sum_{j=0}^{\infty} \sum_{k=0}^{\infty} \sum_{l=0}^{\infty} \sum_{m=0}^{\infty} k l^{2} P_{i j k l m} \sum_{i_{1}=1}^{\infty} \sum_{j_{1}=0}^{\infty} \sum_{k_{1}=0}^{\infty} \sum_{l_{1}=0}^{\infty} \sum_{m_{1}=0}^{\infty} k_{1} P_{i_{1} j_{1} k_{1} l_{1} m_{1}} \\
& +k_{t r t} \sum_{i=1}^{\infty} \sum_{j=0}^{\infty} \sum_{k=0}^{\infty} \sum_{l=0}^{\infty} \sum_{m=0}^{\infty}(k-1) k(l+1) P_{i j k l m} \sum_{i_{1}=1}^{\infty} \sum_{j_{1}=0}^{\infty} \sum_{k_{1}=0}^{\infty} \sum_{l_{1}=0}^{\infty} \sum_{m_{1}=0}^{\infty} l_{1} P_{i_{1} j_{1} k_{1} l_{1} m_{1}} \\
& +k_{t r t} \sum_{i=1}^{\infty} \sum_{j=0}^{\infty} \sum_{k=0}^{\infty} \sum_{l=0}^{\infty} \sum_{m=0}^{\infty} k(l-1) l P_{i j k l m} \sum_{i_{1}=1}^{\infty} \sum_{j_{1}=0}^{\infty} \sum_{k_{1}=0}^{\infty} \sum_{l_{1}=0}^{\infty} \sum_{m_{1}=0}^{\infty} k_{1} P_{i_{1} j_{1} k_{1} l_{1} m_{1}} \\
& -k_{t r p} \sum_{i=1}^{\infty} \sum_{j=0}^{\infty} \sum_{k=0}^{\infty} \sum_{l=0}^{\infty} \sum_{m=0}^{\infty} k^{2} l P_{i j k l m} \sum_{i_{1}=1}^{\infty} \sum_{j_{1}=0}^{\infty} \sum_{k_{1}=0}^{\infty} \sum_{l_{1}=0}^{\infty} \sum_{m_{1}=0}^{\infty} i_{1} P_{i_{1} j_{1} k_{1} l_{1} m_{1}} \\
& -k_{t r p} \sum_{i=1}^{\infty} \sum_{j=0}^{\infty} \sum_{k=0}^{\infty} \sum_{l=0}^{\infty} \sum_{m=0}^{\infty} i k l P_{i j k l m} \sum_{i_{1}=1}^{\infty} \sum_{j_{1}=0}^{\infty} \sum_{k_{1}=0}^{\infty} \sum_{l_{1}=0}^{\infty} \sum_{m_{1}=0}^{\infty} k_{1} P_{i_{1} j_{1} k_{1} l_{1} m_{1}} \\
& +k_{t r p} \sum_{i=1}^{\infty} \sum_{j=0}^{\infty} \sum_{k=0}^{\infty} \sum_{l=0}^{\infty} \sum_{m=0}^{\infty}(k-1) k l P_{i j k l m} \sum_{i_{1}=1}^{\infty} \sum_{j_{1}=0}^{\infty} \sum_{k_{1}=0}^{\infty} \sum_{l_{1}=0}^{\infty} \sum_{m_{1}=0}^{\infty} i_{1} P_{i_{1} j_{1} k_{1} l_{1} m_{1}} \\
& +k_{t r p} \sum_{i=1}^{\infty} \sum_{j=0}^{\infty} \sum_{k=0}^{\infty} \sum_{l=0}^{\infty} \sum_{m=0}^{\infty} i(k+1) l P_{i j k l m} \sum_{i_{1}=1}^{\infty} \sum_{j_{1}=0}^{\infty} \sum_{k_{1}=0}^{\infty} \sum_{l_{1}=0}^{\infty} \sum_{m_{1}=0}^{\infty} k_{1} P_{i_{1} j_{1} k_{1} l_{1} m_{1}}
\end{aligned}
$$




$$
\begin{aligned}
& -k_{t r p} \sum_{i=1}^{\infty} \sum_{j=0}^{\infty} \sum_{k=0}^{\infty} \sum_{l=0}^{\infty} \sum_{m=0}^{\infty} k l m P_{i j k l m} \sum_{i_{1}=1}^{\infty} \sum_{j_{1}=0}^{\infty} \sum_{k_{1}=0}^{\infty} \sum_{l_{1}=0}^{\infty} \sum_{m_{1}=0}^{\infty} i_{1} P_{i_{1} j_{1} k_{1} l_{1} m_{1}} \\
& -k_{t r p} \sum_{i=1}^{\infty} \sum_{j=0}^{\infty} \sum_{k=0}^{\infty} \sum_{l=0}^{\infty} \sum_{m=0}^{\infty} i k l P_{i j k l m} \sum_{i_{1}=1}^{\infty} \sum_{j_{1}=0}^{\infty} \sum_{k_{1}=0}^{\infty} \sum_{l_{1}=0}^{\infty} \sum_{m_{1}=0}^{\infty} m_{1} P_{i_{1} j_{1} k_{1} l_{1} m_{1}} \\
& +k_{t r p} \sum_{i=1}^{\infty} \sum_{j=0}^{\infty} \sum_{k=0}^{\infty} \sum_{l=0}^{\infty} \sum_{m=0}^{\infty} k \operatorname{lm} P_{i j k l m} \sum_{i_{1}=1}^{\infty} \sum_{j_{1}=0}^{\infty} \sum_{k_{1}=0}^{\infty} \sum_{l_{1}=0}^{\infty} \sum_{m_{1}=0}^{\infty} i_{1} P_{i_{1} j_{1} k_{1} l_{1} m_{1}} \\
& +k_{t r p} \sum_{i=1}^{\infty} \sum_{j=0}^{\infty} \sum_{k=0}^{\infty} \sum_{l=0}^{\infty} \sum_{m=0}^{\infty} i k l P_{i j k l m} \sum_{i_{1}=1}^{\infty} \sum_{j_{1}=0}^{\infty} \sum_{k_{1}=0}^{\infty} \sum_{l_{1}=0}^{\infty} \sum_{m_{1}=0}^{\infty} m_{1} P_{i_{1} j_{1} k_{1} l_{1} m_{1}} \\
& -k_{\text {intr }} \sum_{i=1}^{\infty} \sum_{j=0}^{\infty} \sum_{k=0}^{\infty} \sum_{l=0}^{\infty} \sum_{m=0}^{\infty} k^{2} l P_{i j k l m} \\
& +k_{\text {intr }} \sum_{i=1}^{\infty} \sum_{j=0}^{\infty} \sum_{k=0}^{\infty} \sum_{l=0}^{\infty} \sum_{m=0}^{\infty} k^{2} l P_{i j k l m} \\
& -k_{r} \sum_{i=1}^{\infty} \sum_{j=0}^{\infty} \sum_{k=0}^{\infty} \sum_{l=0}^{\infty} \sum_{m=0}^{\infty} k l m P_{i j k l m} \\
& +k_{r} \sum_{i=1}^{\infty} \sum_{j=0}^{\infty} \sum_{k=0}^{\infty} \sum_{l=0}^{\infty} \sum_{m=0}^{\infty}(k+1) \operatorname{lm} P_{i j k l m} \\
& -k_{t c} \sum_{i=1}^{\infty} \sum_{j=0}^{\infty} \sum_{k=0}^{\infty} \sum_{l=0}^{\infty} \sum_{m=0}^{\infty} k^{2} l P_{i j k l m} \sum_{i_{1}=1}^{\infty} \sum_{j_{1}=0}^{\infty} \sum_{k_{1}=0}^{\infty} \sum_{l_{1}=0}^{\infty} \sum_{m_{1}=0}^{\infty} k_{1} P_{i_{1} j_{1} k_{1} l_{1} m_{1}} \\
& +\frac{1}{2}(2) k_{t c} \sum_{i=1}^{\infty} \sum_{j=0}^{\infty} \sum_{k=0}^{\infty} \sum_{l=0}^{\infty} \sum_{m=0}^{\infty} k^{2} l P_{i j k l m} \sum_{i_{1}=1}^{\infty} \sum_{j_{1}=0}^{\infty} \sum_{k_{1}=0}^{\infty} \sum_{l_{1}=0}^{\infty} \sum_{m_{1}=0}^{\infty} k_{1} P_{i_{1} j_{1} k_{1} l_{1} m_{1}} \\
& -\frac{1}{2}(2) k_{t c} \sum_{i=1}^{\infty} \sum_{j=0}^{\infty} \sum_{k=0}^{\infty} \sum_{l=0}^{\infty} \sum_{m=0}^{\infty} k l P_{i j k l m} \sum_{i_{1}=1}^{\infty} \sum_{j_{1}=0}^{\infty} \sum_{k_{1}=0}^{\infty} \sum_{l_{1}=0}^{\infty} \sum_{m_{1}=0}^{\infty} k_{1} P_{i_{1} j_{1} k_{1} l_{1} m_{1}} \\
& -k_{t c} \sum_{i=1}^{\infty} \sum_{j=0}^{\infty} \sum_{k=0}^{\infty} \sum_{l=0}^{\infty} \sum_{m=0}^{\infty} k l m P_{i j k l m} \sum_{i_{1}=1}^{\infty} \sum_{j_{1}=0}^{\infty} \sum_{k_{1}=0}^{\infty} \sum_{l_{1}=0}^{\infty} \sum_{m_{1}=0}^{\infty} m_{1} P_{i_{1} j_{1} k_{1} l_{1} m_{1}} \\
& +\frac{1}{2}(2) k_{t c} \sum_{i=1}^{\infty} \sum_{j=0}^{\infty} \sum_{k=0}^{\infty} \sum_{l=0}^{\infty} \sum_{m=0}^{\infty} k l m P_{i j k l m} \sum_{i_{1}=1}^{\infty} \sum_{j_{1}=0}^{\infty} \sum_{k_{1}=0}^{\infty} \sum_{l_{1}=0}^{\infty} \sum_{m_{1}=0}^{\infty} m_{1} P_{i_{1} j_{1} k_{1} l_{1} m_{1}} \\
& -k_{t c} \sum_{i=1}^{\infty} \sum_{j=0}^{\infty} \sum_{k=0}^{\infty} \sum_{l=0}^{\infty} \sum_{m=0}^{\infty} k^{2} l P_{i j k l m} \sum_{i_{1}=1}^{\infty} \sum_{j_{1}=0}^{\infty} \sum_{k_{1}=0}^{\infty} \sum_{l_{1}=0}^{\infty} \sum_{m_{1}=0}^{\infty} m_{1} P_{i_{1} j_{1} k_{1} l_{1} m_{1}} \\
& -k_{t c} \sum_{i=1}^{\infty} \sum_{j=0}^{\infty} \sum_{k=0}^{\infty} \sum_{l=0}^{\infty} \sum_{m=0}^{\infty} k \operatorname{lm} P_{i j k l m} \sum_{i_{1}=1}^{\infty} \sum_{j_{1}=0}^{\infty} \sum_{k_{1}=0}^{\infty} \sum_{l_{1}=0}^{\infty} \sum_{m_{1}=0}^{\infty} k_{1} P_{i_{1} j_{1} k_{1} l_{1} m_{1}}
\end{aligned}
$$




$$
\begin{aligned}
& +\frac{1}{2}(2) k_{t c} \sum_{i=1}^{\infty} \sum_{j=0}^{\infty} \sum_{k=0}^{\infty} \sum_{l=0}^{\infty} \sum_{m=0}^{\infty} k^{2} l P_{i j k l m} \sum_{i_{1}=1}^{\infty} \sum_{j_{1}=0}^{\infty} \sum_{k_{1}=0}^{\infty} \sum_{l_{1}=0}^{\infty} \sum_{m_{1}=0}^{\infty} m_{1} P_{i_{1} j_{1} k_{1} l_{1} m_{1}} \\
& +\frac{1}{2}(2) k_{t c} \sum_{i=1}^{\infty} \sum_{j=0}^{\infty} \sum_{k=0}^{\infty} \sum_{l=0}^{\infty} \sum_{m=0}^{\infty} k l m P_{i j k l m} \sum_{i_{1}=1}^{\infty} \sum_{j_{1}=0}^{\infty} \sum_{k_{1}=0}^{\infty} \sum_{l_{1}=0}^{\infty} \sum_{m_{1}=0}^{\infty} k_{1} P_{i_{1} j_{1} k_{1} l_{1} m_{1}} \\
& -\frac{1}{2} k_{t c} \sum_{i=1}^{\infty} \sum_{j=0}^{\infty} \sum_{k=0}^{\infty} \sum_{l=0}^{\infty} \sum_{m=0}^{\infty} k l P_{i j k l m} \sum_{i_{1}=1}^{\infty} \sum_{j_{1}=0}^{\infty} \sum_{k_{1}=0}^{\infty} \sum_{l_{1}=0}^{\infty} \sum_{m_{1}=0}^{\infty} m_{1} P_{i_{1} j_{1} k_{1} l_{1} m_{1}} \\
& -\frac{1}{2} k_{t c} \sum_{i=1}^{\infty} \sum_{j=0}^{\infty} \sum_{k=0}^{\infty} \sum_{l=0}^{\infty} \sum_{m=0}^{\infty} l m P_{i j k l m} \sum_{i_{1}=1}^{\infty} \sum_{j_{1}=0}^{\infty} \sum_{k_{1}=0}^{\infty} \sum_{l_{1}=0}^{\infty} \sum_{m_{1}=0}^{\infty} k_{1} P_{i_{1} j_{1} k_{1} l_{1} m_{1}}
\end{aligned}
$$

using Equations (73), (74), (75) and (76).

$$
\begin{aligned}
& \Rightarrow \frac{d \mu_{00110}}{d t}=k_{t r t} C_{T} \sum_{i=1}^{\infty} \sum_{j=0}^{\infty} \sum_{k=0}^{\infty} \sum_{l=0}^{\infty} \sum_{m=0}^{\infty}\left(k^{2}-k l-k\right) P_{i j k l m} \\
& +k_{t r t} \sum_{i=1}^{\infty} \sum_{j=0}^{\infty} \sum_{k=0}^{\infty} \sum_{l=0}^{\infty} \sum_{m=0}^{\infty}\left(k^{2}-k l-k\right) P_{i j k l m} \sum_{i_{1}=1}^{\infty} \sum_{j_{1}=0}^{\infty} \sum_{k_{1}=0}^{\infty} \sum_{l_{1}=0}^{\infty} \sum_{m_{1}=0}^{\infty} l_{1} P_{i_{1} j_{1} k_{1} l_{1} m_{1}} \\
& -k_{t r t} \sum_{i=1}^{\infty} \sum_{j=0}^{\infty} \sum_{k=0}^{\infty} \sum_{l=0}^{\infty} \sum_{m=0}^{\infty} k l P_{i j k l m} \sum_{i_{1}=1}^{\infty} \sum_{j_{1}=0}^{\infty} \sum_{k_{1}=0}^{\infty} \sum_{l_{1}=0}^{\infty} \sum_{m_{1}=0}^{\infty} k_{1} P_{i_{1} j_{1} k_{1} l_{1} m_{1}} \\
& -k_{t r p} \sum_{i=1}^{\infty} \sum_{j=0}^{\infty} \sum_{k=0}^{\infty} \sum_{l=0}^{\infty} \sum_{m=0}^{\infty} k l P_{i j k l m} \sum_{i_{1}=1}^{\infty} \sum_{j_{1}=0}^{\infty} \sum_{k_{1}=0}^{\infty} \sum_{l_{1}=0}^{\infty} \sum_{m_{1}=0}^{\infty} i_{1} P_{i_{1} j_{1} k_{1} l_{1} m_{1}} \\
& +k_{t r p} \sum_{i=1}^{\infty} \sum_{j=0}^{\infty} \sum_{k=0}^{\infty} \sum_{l=0}^{\infty} \sum_{m=0}^{\infty} i l P_{i j k l m} \sum_{i_{1}=1}^{\infty} \sum_{j_{1}=0}^{\infty} \sum_{k_{1}=0}^{\infty} \sum_{l_{1}=0}^{\infty} \sum_{m_{1}=0}^{\infty} k_{1} P_{i_{1} j_{1} k_{1} l_{1} m_{1}} \\
& +k_{r} \sum_{i=1}^{\infty} \sum_{j=0}^{\infty} \sum_{k=0}^{\infty} \sum_{l=0}^{\infty} \sum_{m=0}^{\infty} l m P_{i j k l m} \\
& -k_{t c} \sum_{i=1}^{\infty} \sum_{j=0}^{\infty} \sum_{k=0}^{\infty} \sum_{l=0}^{\infty} \sum_{m=0}^{\infty} k l P_{i j k l m} \sum_{i_{1}=1}^{\infty} \sum_{j_{1}=0}^{\infty} \sum_{k_{1}=0}^{\infty} \sum_{l_{1}=0}^{\infty} \sum_{m_{1}=0}^{\infty} k_{1} P_{i_{1} j_{1} k_{1} l_{1} m_{1}} \\
& -\frac{1}{2} k_{t c} \sum_{i=1}^{\infty} \sum_{j=0}^{\infty} \sum_{k=0}^{\infty} \sum_{l=0}^{\infty} \sum_{m=0}^{\infty} k l P_{i j k l m} \sum_{i_{1}=1}^{\infty} \sum_{j_{1}=0}^{\infty} \sum_{k_{1}=0}^{\infty} \sum_{l_{1}=0}^{\infty} \sum_{m_{1}=0}^{\infty} m_{1} P_{i_{1} j_{1} k_{1} l_{1} m_{1}} \\
& -\frac{1}{2} k_{t c} \sum_{i=1}^{\infty} \sum_{j=0}^{\infty} \sum_{k=0}^{\infty} \sum_{l=0}^{\infty} \sum_{m=0}^{\infty} \operatorname{lm} P_{i j k l m} \sum_{i_{1}=1}^{\infty} \sum_{j_{1}=0}^{\infty} \sum_{k_{1}=0}^{\infty} \sum_{l_{1}=0}^{\infty} \sum_{m_{1}=0}^{\infty} k_{1} P_{i_{1} j_{1} k_{1} l_{1} m_{1}} \\
& \Rightarrow \frac{d \mu_{00110}}{d t}=k_{t r t}\left(C_{T}+\mu_{00010}\right)\left(\mu_{00200}-\mu_{00110}-\mu_{00100}\right) \\
& -k_{t r t} \mu_{00100} \mu_{00110}-k_{t r p} \mu_{00110} \mu_{10000}+k_{t r p} \mu_{10010} \mu_{00100}+k_{r} \mu_{00011} \\
& -k_{t c} \mu_{00110} \mu_{00100}-\frac{1}{2} \mu_{00110} \mu_{00001}-\frac{1}{2} \mu_{00011} \mu_{00100}
\end{aligned}
$$


3.3.9 Second moment w.r.t. the number of inactive (capped) radicals and unrelaxed (just uncapped) radicals

$$
\begin{aligned}
& \frac{d \mu_{00011}}{d t}=\sum_{i=1}^{\infty} \sum_{j=0}^{\infty} \sum_{k=0}^{\infty} \sum_{l=0}^{\infty} \sum_{m=0}^{\infty} \operatorname{lm} \frac{d P_{i j k l m}}{d t} \\
& =-k_{p} C_{M} \sum_{i=1}^{\infty} \sum_{j=0}^{\infty} \sum_{k=0}^{\infty} \sum_{l=0}^{\infty} \sum_{m=0}^{\infty} k l m P_{i j k l m} \\
& +k_{p} C_{M} \sum_{i=2}^{\infty} \sum_{j=0}^{\infty} \sum_{k=0}^{\infty} \sum_{l=0}^{\infty} \sum_{m=0}^{\infty} k l m P_{i-1 j k l m} \\
& -k_{p} C_{M} \sum_{i=1}^{\infty} \sum_{j=0}^{\infty} \sum_{k=0}^{\infty} \sum_{l=0}^{\infty} \sum_{m=0}^{\infty} l m^{2} P_{i j k l m} \\
& +k_{p} C_{M} \sum_{i=2}^{\infty} \sum_{j=0}^{\infty} \sum_{k=0}^{\infty} \sum_{l=0}^{\infty} \sum_{m=0}^{\infty} l m^{2} P_{i-1 j k l m} \\
& -k_{t r t} C_{T} \sum_{i=1}^{\infty} \sum_{j=0}^{\infty} \sum_{k=0}^{\infty} \sum_{l=0}^{\infty} \sum_{m=0}^{\infty} k l m P_{i j k l m} \\
& +k_{t r t} C_{T} \sum_{i=1}^{\infty} \sum_{j=0}^{\infty} \sum_{k=0}^{\infty} \sum_{l=1}^{\infty} \sum_{m=0}^{\infty}(k+1) l m P_{i j k+1 l-1 m} \\
& -k_{t r t} \sum_{i=1}^{\infty} \sum_{j=0}^{\infty} \sum_{k=0}^{\infty} \sum_{l=0}^{\infty} \sum_{m=0}^{\infty} k l m P_{i j k l m} \sum_{i_{1}=1}^{\infty} \sum_{j_{1}=0}^{\infty} \sum_{k_{1}=0}^{\infty} \sum_{l_{1}=0}^{\infty} \sum_{m_{1}=0}^{\infty} l_{1} P_{i_{1} j_{1} k_{1} l_{1} m_{1}} \\
& \text { - } k_{t r t} \sum_{i=1}^{\infty} \sum_{j=0}^{\infty} \sum_{k=0}^{\infty} \sum_{l=0}^{\infty} \sum_{m=0}^{\infty} l^{2} m P_{i j k l m} \sum_{i_{1}=1}^{\infty} \sum_{j_{1}=0}^{\infty} \sum_{k_{1}=0}^{\infty} \sum_{l_{1}=0}^{\infty} \sum_{m_{1}=0}^{\infty} k_{1} P_{i_{1} j_{1} k_{1} l_{1} m_{1}} \\
& +k_{t r t} \sum_{i=1}^{\infty} \sum_{j=0}^{\infty} \sum_{k=0}^{\infty} \sum_{l=1}^{\infty} \sum_{m=0}^{\infty}(k+1) l m P_{i j k+1 l-1 m} \sum_{i_{1}=1}^{\infty} \sum_{j_{1}=0}^{\infty} \sum_{k_{1}=0}^{\infty} \sum_{l_{1}=0}^{\infty} \sum_{m_{1}=0}^{\infty} l_{1} P_{i_{1} j_{1} k_{1} l_{1} m_{1}} \\
& +k_{t r t} \sum_{i=1}^{\infty} \sum_{j=0}^{\infty} \sum_{k=0}^{\infty} \sum_{l=0}^{\infty} \sum_{m=1}^{\infty} l(l+1) m P_{i j k l+1 m-1} \sum_{i_{1}=1}^{\infty} \sum_{j_{1}=0}^{\infty} \sum_{k_{1}=0}^{\infty} \sum_{l_{1}=0}^{\infty} \sum_{m_{1}=0}^{\infty} k_{1} P_{i_{1} j_{1} k_{1} l_{1} m_{1}} \\
& -k_{t r p} \sum_{i=1}^{\infty} \sum_{j=0}^{\infty} \sum_{k=0}^{\infty} \sum_{l=0}^{\infty} \sum_{m=0}^{\infty} k l m P_{i j k l m} \sum_{i_{1}=1}^{\infty} \sum_{j_{1}=0}^{\infty} \sum_{k_{1}=0}^{\infty} \sum_{l_{1}=0}^{\infty} \sum_{m_{1}=0}^{\infty} i_{1} P_{i_{1} j_{1} k_{1} l_{1} m_{1}} \\
& -k_{t r p} \sum_{i=1}^{\infty} \sum_{j=0}^{\infty} \sum_{k=0}^{\infty} \sum_{l=0}^{\infty} \sum_{m=0}^{\infty} i l m P_{i j k l m} \sum_{i_{1}=1}^{\infty} \sum_{j_{1}=0}^{\infty} \sum_{k_{1}=0}^{\infty} \sum_{l_{1}=0}^{\infty} \sum_{m_{1}=0}^{\infty} k_{1} P_{i_{1} j_{1} k_{1} l_{1} m_{1}} \\
& +k_{t r p} \sum_{i=1}^{\infty} \sum_{j=0}^{\infty} \sum_{k=0}^{\infty} \sum_{l=0}^{\infty} \sum_{m=0}^{\infty}(k+1) l m P_{i j k+1 l m} \sum_{i_{1}=1}^{\infty} \sum_{j_{1}=0}^{\infty} \sum_{k_{1}=0}^{\infty} \sum_{l_{1}=0}^{\infty} \sum_{m_{1}=0}^{\infty} i_{1} P_{i_{1} j_{1} k_{1} l_{1} m_{1}} \\
& +k_{\text {trp }} \sum_{i=1}^{\infty} \sum_{j=1}^{\infty} \sum_{k=1}^{\infty} \sum_{l=0}^{\infty} \sum_{m=0}^{\infty} i l m P_{i j-1 k-1 l m} \sum_{i_{1}=1}^{\infty} \sum_{j_{1}=0}^{\infty} \sum_{k_{1}=0}^{\infty} \sum_{l_{1}=0}^{\infty} \sum_{m_{1}=0}^{\infty} k_{1} P_{i_{1} j_{1} k_{1} l_{1} m_{1}}
\end{aligned}
$$


$-k_{t r p} \sum_{i=1}^{\infty} \sum_{j=0}^{\infty} \sum_{k=0}^{\infty} \sum_{l=0}^{\infty} \sum_{m=0}^{\infty} l m^{2} P_{i j k l m} \sum_{i_{1}=1}^{\infty} \sum_{j_{1}=0}^{\infty} \sum_{k_{1}=0}^{\infty} \sum_{l_{1}=0}^{\infty} \sum_{m_{1}=0}^{\infty} i_{1} P_{i_{1} j_{1} k_{1} l_{1} m_{1}}$

$-k_{t r p} \sum_{i=1}^{\infty} \sum_{j=0}^{\infty} \sum_{k=0}^{\infty} \sum_{l=0}^{\infty} \sum_{m=0}^{\infty} i l m P_{i j k l m} \sum_{i_{1}=1}^{\infty} \sum_{j_{1}=0}^{\infty} \sum_{k_{1}=0}^{\infty} \sum_{l_{1}=0}^{\infty} \sum_{m_{1}=0}^{\infty} m_{1} P_{i_{1} j_{1} k_{1} l_{1} m_{1}}$

$+k_{t r p} \sum_{i=1}^{\infty} \sum_{j=0}^{\infty} \sum_{k=0}^{\infty} \sum_{l=0}^{\infty} \sum_{m=0}^{\infty} \operatorname{lm}(m+1) P_{i j k l m+1} \sum_{i_{1}=1}^{\infty} \sum_{j_{1}=0}^{\infty} \sum_{k_{1}=0}^{\infty} \sum_{l_{1}=0}^{\infty} \sum_{m_{1}=0}^{\infty} i_{1} P_{i_{1} j_{1} k_{1} l_{1} m_{1}}$

$+k_{t r p} \sum_{i=1}^{\infty} \sum_{j=1}^{\infty} \sum_{k=0}^{\infty} \sum_{l=0}^{\infty} \sum_{m=1}^{\infty} i l m P_{i j-1 k l m-1} \sum_{i_{1}=1}^{\infty} \sum_{j_{1}=0}^{\infty} \sum_{k_{1}=0}^{\infty} \sum_{l_{1}=0}^{\infty} \sum_{m_{1}=0}^{\infty} m_{1} P_{i_{1} j_{1} k_{1} l_{1} m_{1}}$

$-k_{\text {intr }} \sum_{i=1}^{\infty} \sum_{j=0}^{\infty} \sum_{k=0}^{\infty} \sum_{l=0}^{\infty} \sum_{m=0}^{\infty} k l m P_{i j k l m}$

$+k_{\text {intr }} \sum_{i=1}^{\infty} \sum_{j=1}^{\infty} \sum_{k=0}^{\infty} \sum_{l=0}^{\infty} \sum_{m=0}^{\infty} k l m P_{i j-1 k l m}$

$-k_{r} \sum_{i=1}^{\infty} \sum_{j=0}^{\infty} \sum_{k=0}^{\infty} \sum_{l=0}^{\infty} \sum_{m=0}^{\infty} l m^{2} P_{i j k l m}$

$+k_{r} \sum_{i=1}^{\infty} \sum_{j=0}^{\infty} \sum_{k=1}^{\infty} \sum_{l=0}^{\infty} \sum_{m=0}^{\infty} \operatorname{lm}(m+1) P_{i j k-1 l m+1}$

$-k_{t c} \sum_{i=1}^{\infty} \sum_{j=0}^{\infty} \sum_{k=0}^{\infty} \sum_{l=0}^{\infty} \sum_{m=0}^{\infty} k l m P_{i j k l m} \sum_{i_{1}=1}^{\infty} \sum_{j_{1}=0}^{\infty} \sum_{k_{1}=0}^{\infty} \sum_{l_{1}=0}^{\infty} \sum_{m_{1}=0}^{\infty} k_{1} P_{i_{1} j_{1} k_{1} l_{1} m_{1}}$

$+\frac{1}{2} k_{t c} \sum_{i=1}^{\infty} \sum_{j=0}^{\infty} \sum_{k=0}^{\infty} \sum_{l=0}^{\infty} \sum_{m=0}^{\infty} \sum_{i_{1}=1}^{i} \sum_{j_{1}=0}^{j} \sum_{k_{1}=0}^{k+2} \sum_{l_{1}=0}^{l} \sum_{m_{1}=0}^{m} l m\left[k_{1} P_{i_{1} j_{1} k_{1} l_{1} m_{1}}\left(k+2-k_{1}\right) P_{i-i_{1}, j-j_{1}, k+2-k_{1}, l-l_{1} m-m_{1}}\right]$

$-k_{t c} \sum_{i=1}^{\infty} \sum_{j=0}^{\infty} \sum_{k=0}^{\infty} \sum_{l=0}^{\infty} \sum_{m=0}^{\infty} l m^{2} P_{i j k l m} \sum_{i_{1}=1}^{\infty} \sum_{j_{1}=0}^{\infty} \sum_{k_{1}=0}^{\infty} \sum_{l_{1}=0}^{\infty} \sum_{m_{1}=0}^{\infty} m_{1} P_{i_{1} j_{1} k_{1} l_{1} m_{1}}$

$+\frac{1}{2} k_{t c} \sum_{i=1}^{\infty} \sum_{j=0}^{\infty} \sum_{k=0}^{\infty} \sum_{l=0}^{\infty} \sum_{m=0}^{\infty} \sum_{i_{1}=1}^{i} \sum_{j_{1}=0}^{j} \sum_{k_{1}=0}^{k} \sum_{l_{1}=0}^{l} \sum_{m_{1}=0}^{m+2}\left[l m m_{1} P_{i_{1} j_{1} k_{1} l_{1} m_{1}}\left(m+2-m_{1}\right) P_{i-i_{1}, j-j_{1}, k-k_{1}, l-l_{1} m+2-m_{1}}\right]$

$-k_{t c} \sum_{i=1}^{\infty} \sum_{j=0}^{\infty} \sum_{k=0}^{\infty} \sum_{l=0}^{\infty} \sum_{m=0}^{\infty} k l m P_{i j k l m} \sum_{i_{1}=1}^{\infty} \sum_{j_{1}=0}^{\infty} \sum_{k_{1}=0}^{\infty} \sum_{l_{1}=0}^{\infty} \sum_{m_{1}=0}^{\infty} m_{1} P_{i_{1} j_{1} k_{1} l_{1} m_{1}}$

$-k_{t c} \sum_{i=1}^{\infty} \sum_{j=0}^{\infty} \sum_{k=0}^{\infty} \sum_{l=0}^{\infty} \sum_{m=0}^{\infty} l m^{2} P_{i j k l m} \sum_{i_{1}=1}^{\infty} \sum_{j_{1}=0}^{\infty} \sum_{k_{1}=0}^{\infty} \sum_{l_{1}=0}^{\infty} \sum_{m_{1}=0}^{\infty} k_{1} P_{i_{1} j_{1} k_{1} l_{1} m_{1}}$

$+\frac{1}{2} k_{t c} \sum_{i=1}^{\infty} \sum_{j=0}^{\infty} \sum_{k=0}^{\infty} \sum_{l=0}^{\infty} \sum_{m=0}^{\infty} \sum_{i_{1}=1}^{i} \sum_{j_{1}=0}^{j} \sum_{k_{1}=0}^{k+1} \sum_{l_{1}=0}^{l} \sum_{m_{1}=0}^{m+1}\left[l m k_{1} P_{i_{1} j_{1} k_{1} l_{1} m_{1}}\left(m+1-m_{1}\right) P_{i-i_{1}, j-j_{1}, k+1-k_{1}, l-l_{1} m+1-m_{1}}\right]$

$+\frac{1}{2} k_{t c} \sum_{i=1}^{\infty} \sum_{j=0}^{\infty} \sum_{k=0}^{\infty} \sum_{l=0}^{\infty} \sum_{m=0}^{\infty} \sum_{i_{1}=1}^{i} \sum_{j_{1}=0}^{j} \sum_{k_{1}=0}^{k+1} \sum_{l_{1}=0}^{l} \sum_{m_{1}=0}^{m+1}\left[l m m_{1} P_{i_{1} j_{1} k_{1} l_{1} m_{1}}\left(k+1-k_{1}\right) P_{i-i_{1}, j-j_{1}, k+1-k_{1}, l-l_{1} m+1-m_{1}}\right]$ 


$$
\begin{aligned}
& \Rightarrow \frac{d \mu_{00011}}{d t}=-k_{p} C_{M} \sum_{i=1}^{\infty} \sum_{j=0}^{\infty} \sum_{k=0}^{\infty} \sum_{l=0}^{\infty} \sum_{m=0}^{\infty} k l m P_{i j k l m} \\
& +k_{p} C_{M} \sum_{i=1}^{\infty} \sum_{j=0}^{\infty} \sum_{k=0}^{\infty} \sum_{l=0}^{\infty} \sum_{m=0}^{\infty} k l m P_{i j k l m} \\
& -k_{p} C_{M} \sum_{i=1}^{\infty} \sum_{j=0}^{\infty} \sum_{k=0}^{\infty} \sum_{l=0}^{\infty} \sum_{m=0}^{\infty} l m^{2} P_{i j k l m} \\
& +k_{p} C_{M} \sum_{i=1}^{\infty} \sum_{j=0}^{\infty} \sum_{k=0}^{\infty} \sum_{l=0}^{\infty} \sum_{m=0}^{\infty} l m^{2} P_{i j k l m} \\
& -k_{t r t} C_{T} \sum_{i=1}^{\infty} \sum_{j=0}^{\infty} \sum_{k=0}^{\infty} \sum_{l=0}^{\infty} \sum_{m=0}^{\infty} k \operatorname{lm} P_{i j k l m} \\
& +k_{t r t} C_{T} \sum_{i=1}^{\infty} \sum_{j=0}^{\infty} \sum_{k=0}^{\infty} \sum_{l=0}^{\infty} \sum_{m=0}^{\infty} k(l+1) m P_{i j k l m} \\
& \text { - } k_{t r t} \sum_{i=1}^{\infty} \sum_{j=0}^{\infty} \sum_{k=0}^{\infty} \sum_{l=0}^{\infty} \sum_{m=0}^{\infty} k l m P_{i j k l m} \sum_{i_{1}=1}^{\infty} \sum_{j_{1}=0}^{\infty} \sum_{k_{1}=0}^{\infty} \sum_{l_{1}=0}^{\infty} \sum_{m_{1}=0}^{\infty} l_{1} P_{i_{1} j_{1} k_{1} l_{1} m_{1}} \\
& -k_{t r t} \sum_{i=1}^{\infty} \sum_{j=0}^{\infty} \sum_{k=0}^{\infty} \sum_{l=0}^{\infty} \sum_{m=0}^{\infty} l^{2} m P_{i j k l m} \sum_{i_{1}=1}^{\infty} \sum_{j_{1}=0}^{\infty} \sum_{k_{1}=0}^{\infty} \sum_{l_{1}=0}^{\infty} \sum_{m_{1}=0}^{\infty} k_{1} P_{i_{1} j_{1} k_{1} l_{1} m_{1}} \\
& +k_{t r t} \sum_{i=1}^{\infty} \sum_{j=0}^{\infty} \sum_{k=0}^{\infty} \sum_{l=0}^{\infty} \sum_{m=0}^{\infty} k(l+1) m P_{i j k l m} \sum_{i_{1}=1}^{\infty} \sum_{j_{1}=0}^{\infty} \sum_{k_{1}=0}^{\infty} \sum_{l_{1}=0}^{\infty} \sum_{m_{1}=0}^{\infty} l_{1} P_{i_{1} j_{1} k_{1} l_{1} m_{1}} \\
& +k_{t r t} \sum_{i=1}^{\infty} \sum_{j=0}^{\infty} \sum_{k=0}^{\infty} \sum_{l=0}^{\infty} \sum_{m=0}^{\infty}(l-1) l(m+1) P_{i j k l m} \sum_{i_{1}=1}^{\infty} \sum_{j_{1}=0}^{\infty} \sum_{k_{1}=0}^{\infty} \sum_{l_{1}=0}^{\infty} \sum_{m_{1}=0}^{\infty} k_{1} P_{i_{1} j_{1} k_{1} l_{1} m_{1}} \\
& -k_{t r p} \sum_{i=1}^{\infty} \sum_{j=0}^{\infty} \sum_{k=0}^{\infty} \sum_{l=0}^{\infty} \sum_{m=0}^{\infty} k l m P_{i j k l m} \sum_{i_{1}=1}^{\infty} \sum_{j_{1}=0}^{\infty} \sum_{k_{1}=0}^{\infty} \sum_{l_{1}=0}^{\infty} \sum_{m_{1}=0}^{\infty} i_{1} P_{i_{1} j_{1} k_{1} l_{1} m_{1}} \\
& -k_{t r p} \sum_{i=1}^{\infty} \sum_{j=0}^{\infty} \sum_{k=0}^{\infty} \sum_{l=0}^{\infty} \sum_{m=0}^{\infty} i \operatorname{lm} P_{i j k l m} \sum_{i_{1}=1}^{\infty} \sum_{j_{1}=0}^{\infty} \sum_{k_{1}=0}^{\infty} \sum_{l_{1}=0}^{\infty} \sum_{m_{1}=0}^{\infty} k_{1} P_{i_{1} j_{1} k_{1} l_{1} m_{1}} \\
& +k_{t r p} \sum_{i=1}^{\infty} \sum_{j=0}^{\infty} \sum_{k=0}^{\infty} \sum_{l=0}^{\infty} \sum_{m=0}^{\infty} k l m P_{i j k l m} \sum_{i_{1}=1}^{\infty} \sum_{j_{1}=0}^{\infty} \sum_{k_{1}=0}^{\infty} \sum_{l_{1}=0}^{\infty} \sum_{m_{1}=0}^{\infty} i_{1} P_{i_{1} j_{1} k_{1} l_{1} m_{1}} \\
& +k_{t r p} \sum_{i=1}^{\infty} \sum_{j=0}^{\infty} \sum_{k=0}^{\infty} \sum_{l=0}^{\infty} \sum_{m=0}^{\infty} i l m P_{i j k l m} \sum_{i_{1}=1}^{\infty} \sum_{j_{1}=0}^{\infty} \sum_{k_{1}=0}^{\infty} \sum_{l_{1}=0}^{\infty} \sum_{m_{1}=0}^{\infty} k_{1} P_{i_{1} j_{1} k_{1} l_{1} m_{1}}
\end{aligned}
$$




$$
\begin{aligned}
& -k_{\operatorname{trp}} \sum_{i=1}^{\infty} \sum_{j=0}^{\infty} \sum_{k=0}^{\infty} \sum_{l=0}^{\infty} \sum_{m=0}^{\infty} l m^{2} P_{i j k l m} \sum_{i_{1}=1}^{\infty} \sum_{j_{1}=0}^{\infty} \sum_{k_{1}=0}^{\infty} \sum_{l_{1}=0}^{\infty} \sum_{m_{1}=0}^{\infty} i_{1} P_{i_{1} j_{1} k_{1} l_{1} m_{1}} \\
& -k_{\operatorname{trp}} \sum_{i=1}^{\infty} \sum_{j=0}^{\infty} \sum_{k=0}^{\infty} \sum_{l=0}^{\infty} \sum_{m=0}^{\infty} i \operatorname{lm} P_{i j k l m} \sum_{i_{1}=1}^{\infty} \sum_{j_{1}=0}^{\infty} \sum_{k_{1}=0}^{\infty} \sum_{l_{1}=0}^{\infty} \sum_{m_{1}=0}^{\infty} m_{1} P_{i_{1} j_{1} k_{1} l_{1} m_{1}} \\
& +k_{\text {trp }} \sum_{i=1}^{\infty} \sum_{j=0}^{\infty} \sum_{k=0}^{\infty} \sum_{l=0}^{\infty} \sum_{m=0}^{\infty} l(m-1) m P_{i j k l m} \sum_{i_{1}=1}^{\infty} \sum_{j_{1}=0}^{\infty} \sum_{k_{1}=0}^{\infty} \sum_{l_{1}=0}^{\infty} \sum_{m_{1}=0}^{\infty} i_{1} P_{i_{1} j_{1} k_{1} l_{1} m_{1}} \\
& +k_{t r p} \sum_{i=1}^{\infty} \sum_{j=0}^{\infty} \sum_{k=0}^{\infty} \sum_{l=0}^{\infty} \sum_{m=0}^{\infty} i l(m+1) P_{i j k l m} \sum_{i_{1}=1}^{\infty} \sum_{j_{1}=0}^{\infty} \sum_{k_{1}=0}^{\infty} \sum_{l_{1}=0}^{\infty} \sum_{m_{1}=0}^{\infty} m_{1} P_{i_{1} j_{1} k_{1} l_{1} m_{1}} \\
& -k_{i n t r} \sum_{i=1}^{\infty} \sum_{j=0}^{\infty} \sum_{k=0}^{\infty} \sum_{l=0}^{\infty} \sum_{m=0}^{\infty} k l m P_{i j k l m} \\
& +k_{i n t r} \sum_{i=1}^{\infty} \sum_{j=0}^{\infty} \sum_{k=0}^{\infty} \sum_{l=0}^{\infty} \sum_{m=0}^{\infty} k l m P_{i j k l m} \\
& -k_{r} \sum_{i=1}^{\infty} \sum_{j=0}^{\infty} \sum_{k=0}^{\infty} \sum_{l=0}^{\infty} \sum_{m=0}^{\infty} l m^{2} P_{i j k l m} \\
& +k_{r} \sum_{i=1}^{\infty} \sum_{j=0}^{\infty} \sum_{k=0}^{\infty} \sum_{l=0}^{\infty} \sum_{m=0}^{\infty} l(m-1) m P_{i j k l m} \\
& -k_{t c} \sum_{i=1}^{\infty} \sum_{j=0}^{\infty} \sum_{k=0}^{\infty} \sum_{l=0}^{\infty} \sum_{m=0}^{\infty} k l m P_{i j k l m} \sum_{i_{1}=1}^{\infty} \sum_{j_{1}=0}^{\infty} \sum_{k_{1}=0}^{\infty} \sum_{l_{1}=0}^{\infty} \sum_{m_{1}=0}^{\infty} k_{1} P_{i_{1} j_{1} k_{1} l_{1} m_{1}} \\
& +\frac{1}{2}(2) k_{t c} \sum_{i=1}^{\infty} \sum_{j=0}^{\infty} \sum_{k=0}^{\infty} \sum_{l=0}^{\infty} \sum_{m=0}^{\infty} k l m P_{i j k l m} \sum_{i_{1}=1}^{\infty} \sum_{j_{1}=0}^{\infty} \sum_{k_{1}=0}^{\infty} \sum_{l_{1}=0}^{\infty} \sum_{m_{1}=0}^{\infty} k_{1} P_{i_{1} j_{1} k_{1} l_{1} m_{1}} \\
& -k_{t c} \sum_{i=1}^{\infty} \sum_{j=0}^{\infty} \sum_{k=0}^{\infty} \sum_{l=0}^{\infty} \sum_{m=0}^{\infty} l m^{2} P_{i j k l m} \sum_{i_{1}=1}^{\infty} \sum_{j_{1}=0}^{\infty} \sum_{k_{1}=0}^{\infty} \sum_{l_{1}=0}^{\infty} \sum_{m_{1}=0}^{\infty} m_{1} P_{i_{1} j_{1} k_{1} l_{1} m_{1}} \\
& +\frac{1}{2}(2) k_{t c} \sum_{i=1}^{\infty} \sum_{j=0}^{\infty} \sum_{k=0}^{\infty} \sum_{l=0}^{\infty} \sum_{m=0}^{\infty} l m^{2} P_{i j k l m} \sum_{i_{1}=1}^{\infty} \sum_{j_{1}=0}^{\infty} \sum_{k_{1}=0}^{\infty} \sum_{l_{1}=0}^{\infty} \sum_{m_{1}=0}^{\infty} m_{1} P_{i_{1} j_{1} k_{1} l_{1} m_{1}} \\
& -\frac{1}{2}(2) k_{t c} \sum_{i=1}^{\infty} \sum_{j=0}^{\infty} \sum_{k=0}^{\infty} \sum_{l=0}^{\infty} \sum_{m=0}^{\infty} l m P_{i j k l m} \sum_{i_{1}=1}^{\infty} \sum_{j_{1}=0}^{\infty} \sum_{k_{1}=0}^{\infty} \sum_{l_{1}=0}^{\infty} \sum_{m_{1}=0}^{\infty} m_{1} P_{i_{1} j_{1} k_{1} l_{1} m_{1}} \\
& -k_{t c} \sum_{i=1}^{\infty} \sum_{j=0}^{\infty} \sum_{k=0}^{\infty} \sum_{l=0}^{\infty} \sum_{m=0}^{\infty} k l m P_{i j k l m} \sum_{i_{1}=1}^{\infty} \sum_{j_{1}=0}^{\infty} \sum_{k_{1}=0}^{\infty} \sum_{l_{1}=0}^{\infty} \sum_{m_{1}=0}^{\infty} m_{1} P_{i_{1} j_{1} k_{1} l_{1} m_{1}} \\
& -k_{t c} \sum_{i=1}^{\infty} \sum_{j=0}^{\infty} \sum_{k=0}^{\infty} \sum_{l=0}^{\infty} \sum_{m=0}^{\infty} l m^{2} P_{i j k l m} \sum_{i_{1}=1}^{\infty} \sum_{j_{1}=0}^{\infty} \sum_{k_{1}=0}^{\infty} \sum_{l_{1}=0}^{\infty} \sum_{m_{1}=0}^{\infty} k_{1} P_{i_{1} j_{1} k_{1} l_{1} m_{1}}
\end{aligned}
$$




$$
\begin{aligned}
& +\frac{1}{2}(2) k_{t c} \sum_{i=1}^{\infty} \sum_{j=0}^{\infty} \sum_{k=0}^{\infty} \sum_{l=0}^{\infty} \sum_{m=0}^{\infty} k l m P_{i j k l m} \sum_{i_{1}=1}^{\infty} \sum_{j_{1}=0}^{\infty} \sum_{k_{1}=0}^{\infty} \sum_{l_{1}=0}^{\infty} \sum_{m_{1}=0}^{\infty} m_{1} P_{i_{1} j_{1} k_{1} l_{1} m_{1}} \\
& +\frac{1}{2}(2) k_{t c} \sum_{i=1}^{\infty} \sum_{j=0}^{\infty} \sum_{k=0}^{\infty} \sum_{l=0}^{\infty} \sum_{m=0}^{\infty} l_{m}^{2} P_{i j k l m} \sum_{i_{1}=1}^{\infty} \sum_{j_{1}=0}^{\infty} \sum_{k_{1}=0}^{\infty} \sum_{l_{1}=0}^{\infty} \sum_{m_{1}=0}^{\infty} k_{1} P_{i_{1} j_{1} k_{1} l_{1} m_{1}} \\
& -\frac{1}{2} k_{t c} \sum_{i=1}^{\infty} \sum_{j=0}^{\infty} \sum_{k=0}^{\infty} \sum_{l=0}^{\infty} \sum_{m=0}^{\infty} k l P_{i j k l m} \sum_{i_{1}=1}^{\infty} \sum_{j_{1}=0}^{\infty} \sum_{k_{1}=0}^{\infty} \sum_{l_{1}=0}^{\infty} \sum_{m_{1}=0}^{\infty} m_{1} P_{i_{1} j_{1} k_{1} l_{1} m_{1}} \\
& -\frac{1}{2} k_{t c} \sum_{i=1}^{\infty} \sum_{j=0}^{\infty} \sum_{k=0}^{\infty} \sum_{l=0}^{\infty} \sum_{m=0}^{\infty} \operatorname{lm} P_{i j k l m} \sum_{i_{1}=1}^{\infty} \sum_{j_{1}=0}^{\infty} \sum_{k_{1}=0}^{\infty} \sum_{l_{1}=0}^{\infty} \sum_{m_{1}=0}^{\infty} k_{1} P_{i_{1} j_{1} k_{1} l_{1} m_{1}}
\end{aligned}
$$

using Equations (73), (74), (75) and (76).

$$
\begin{aligned}
& \Rightarrow \frac{d \mu_{00011}}{d t}=k_{t r t} C_{T} \sum_{i=1}^{\infty} \sum_{j=0}^{\infty} \sum_{k=0}^{\infty} \sum_{l=0}^{\infty} \sum_{m=0}^{\infty} k m P_{i j k l m} \\
& +k_{t r t} \sum_{i=1}^{\infty} \sum_{j=0}^{\infty} \sum_{k=0}^{\infty} \sum_{l=0}^{\infty} \sum_{m=0}^{\infty} k m P_{i j k l m} \sum_{i_{1}=1}^{\infty} \sum_{j_{1}=0}^{\infty} \sum_{k_{1}=0}^{\infty} \sum_{l_{1}=0}^{\infty} \sum_{m_{1}=0}^{\infty} l_{1} P_{i_{1} j_{1} k_{1} l_{1} m_{1}} \\
& +k_{t r t} \sum_{i=1}^{\infty} \sum_{j=0}^{\infty} \sum_{k=0}^{\infty} \sum_{l=0}^{\infty} \sum_{m=0}^{\infty}\left(l^{2}-l m-l\right) P_{i j k l m} \sum_{i_{1}=1}^{\infty} \sum_{j_{1}=0}^{\infty} \sum_{k_{1}=0}^{\infty} \sum_{l_{1}=0}^{\infty} \sum_{m_{1}=0}^{\infty} k_{1} P_{i_{1} j_{1} k_{1} l_{1} m_{1}} \\
& -k_{t r p} \sum_{i=1}^{\infty} \sum_{j=0}^{\infty} \sum_{k=0}^{\infty} \sum_{l=0}^{\infty} \sum_{m=0}^{\infty} \operatorname{lm} P_{i j k l m} \sum_{i_{1}=1}^{\infty} \sum_{j_{1}=0}^{\infty} \sum_{k_{1}=0}^{\infty} \sum_{l_{1}=0}^{\infty} \sum_{m_{1}=0}^{\infty} i_{1} P_{i_{1} j_{1} k_{1} l_{1} m_{1}} \\
& +k_{t r p} \sum_{i=1}^{\infty} \sum_{j=0}^{\infty} \sum_{k=0}^{\infty} \sum_{l=0}^{\infty} \sum_{m=0}^{\infty} i l P_{i j k l m} \sum_{i_{1}=1}^{\infty} \sum_{j_{1}=0}^{\infty} \sum_{k_{1}=0}^{\infty} \sum_{l_{1}=0}^{\infty} \sum_{m_{1}=0}^{\infty} m_{1} P_{i_{1} j_{1} k_{1} l_{1} m_{1}} \\
& -k_{r} \sum_{i=1}^{\infty} \sum_{j=0}^{\infty} \sum_{k=0}^{\infty} \sum_{l=0}^{\infty} \sum_{m=0}^{\infty} l m P_{i j k l m} \\
& -k_{t c} \sum_{i=1}^{\infty} \sum_{j=0}^{\infty} \sum_{k=0}^{\infty} \sum_{l=0}^{\infty} \sum_{m=0}^{\infty} \operatorname{lm} P_{i j k l m} \sum_{i_{1}=1}^{\infty} \sum_{j_{1}=0}^{\infty} \sum_{k_{1}=0}^{\infty} \sum_{l_{1}=0}^{\infty} \sum_{m_{1}=0}^{\infty} m_{1} P_{i_{1} j_{1} k_{1} l_{1} m_{1}} \\
& -\frac{1}{2} k_{t c} \sum_{i=1}^{\infty} \sum_{j=0}^{\infty} \sum_{k=0}^{\infty} \sum_{l=0}^{\infty} \sum_{m=0}^{\infty} k l P_{i j k l m} \sum_{i_{1}=1}^{\infty} \sum_{j_{1}=0}^{\infty} \sum_{k_{1}=0}^{\infty} \sum_{l_{1}=0}^{\infty} \sum_{m_{1}=0}^{\infty} m_{1} P_{i_{1} j_{1} k_{1} l_{1} m_{1}} \\
& -\frac{1}{2} k_{t c} \sum_{i=1}^{\infty} \sum_{j=0}^{\infty} \sum_{k=0}^{\infty} \sum_{l=0}^{\infty} \sum_{m=0}^{\infty} l m P_{i j k l m} \sum_{i_{1}=1}^{\infty} \sum_{j_{1}=0}^{\infty} \sum_{k_{1}=0}^{\infty} \sum_{l_{1}=0}^{\infty} \sum_{m_{1}=0}^{\infty} k_{1} P_{i_{1} j_{1} k_{1} l_{1} m_{1}} \\
& \frac{d \mu_{00011}}{d t}=k_{t r t}\left(C_{T}+\mu_{00010}\right) \mu_{00101}+k_{t r t} \mu_{00100}\left(\mu_{00020}-\mu_{00011}-\mu_{00001}\right) \\
& -k_{t r p} \mu_{00011} \mu_{10000}+k_{t r p} \mu_{10010} \mu_{00001}-k_{r} \mu_{00011} \\
& -k_{t c} \mu_{00110} \mu_{00001}-\frac{1}{2} k_{t c} \mu_{00110} \mu_{00001}-\frac{1}{2} k_{t c} \mu_{00011} \mu_{00100}
\end{aligned}
$$


3.3.10 Second moment w.r.t. the number of inactive (capped) radicals

$$
\begin{aligned}
& \frac{d \mu_{00020}}{d t}=\sum_{i=1}^{\infty} \sum_{j=0}^{\infty} \sum_{k=0}^{\infty} \sum_{l=0}^{\infty} \sum_{m=0}^{\infty} l^{2} \frac{d P_{i j k l m}}{d t} \\
& =-k_{p} C_{M} \sum_{i=1}^{\infty} \sum_{j=0}^{\infty} \sum_{k=0}^{\infty} \sum_{l=0}^{\infty} \sum_{m=0}^{\infty} k l^{2} P_{i j k l m} \\
& +k_{p} C_{M} \sum_{i=2}^{\infty} \sum_{j=0}^{\infty} \sum_{k=0}^{\infty} \sum_{l=0}^{\infty} \sum_{m=0}^{\infty} k l^{2} P_{i-1 j k l m} \\
& -k_{p} C_{M} \sum_{i=1}^{\infty} \sum_{j=0}^{\infty} \sum_{k=0}^{\infty} \sum_{l=0}^{\infty} \sum_{m=0}^{\infty} l^{2} m P_{i j k l m} \\
& +k_{p} C_{M} \sum_{i=2}^{\infty} \sum_{j=0}^{\infty} \sum_{k=0}^{\infty} \sum_{l=0}^{\infty} \sum_{m=0}^{\infty} l^{2} m P_{i-1 j k l m} \\
& -k_{t r t} C_{T} \sum_{i=1}^{\infty} \sum_{j=0}^{\infty} \sum_{k=0}^{\infty} \sum_{l=0}^{\infty} \sum_{m=0}^{\infty} k l^{2} P_{i j k l m} \\
& +k_{t r t} C_{T} \sum_{i=1}^{\infty} \sum_{j=0}^{\infty} \sum_{k=0}^{\infty} \sum_{l=1}^{\infty} \sum_{m=0}^{\infty}(k+1) l^{2} P_{i j k+1 l-1 m} \\
& -k_{t r t} \sum_{i=1}^{\infty} \sum_{j=0}^{\infty} \sum_{k=0}^{\infty} \sum_{l=0}^{\infty} \sum_{m=0}^{\infty} k l^{2} P_{i j k l m} \sum_{i_{1}=1}^{\infty} \sum_{j_{1}=0}^{\infty} \sum_{k_{1}=0}^{\infty} \sum_{l_{1}=0}^{\infty} \sum_{m_{1}=0}^{\infty} l_{1} P_{i_{1} j_{1} k_{1} l_{1} m_{1}} \\
& -k_{t r t} \sum_{i=1}^{\infty} \sum_{j=0}^{\infty} \sum_{k=0}^{\infty} \sum_{l=0}^{\infty} \sum_{m=0}^{\infty} l^{3} P_{i j k l m} \sum_{i_{1}=1}^{\infty} \sum_{j_{1}=0}^{\infty} \sum_{k_{1}=0}^{\infty} \sum_{l_{1}=0}^{\infty} \sum_{m_{1}=0}^{\infty} k_{1} P_{i_{1} j_{1} k_{1} l_{1} m_{1}} \\
& +k_{t r t} \sum_{i=1}^{\infty} \sum_{j=0}^{\infty} \sum_{k=0}^{\infty} \sum_{l=1}^{\infty} \sum_{m=0}^{\infty}(k+1) l^{2} P_{i j k+1 l-1 m} \sum_{i_{1}=1}^{\infty} \sum_{j_{1}=0}^{\infty} \sum_{k_{1}=0}^{\infty} \sum_{l_{1}=0}^{\infty} \sum_{m_{1}=0}^{\infty} l_{1} P_{i_{1} j_{1} k_{1} l_{1} m_{1}} \\
& +k_{t r t} \sum_{i=1}^{\infty} \sum_{j=0}^{\infty} \sum_{k=0}^{\infty} \sum_{l=0}^{\infty} \sum_{m=1}^{\infty} l^{2}(l+1) P_{i j k l+1 m-1} \sum_{i_{1}=1}^{\infty} \sum_{j_{1}=0}^{\infty} \sum_{k_{1}=0}^{\infty} \sum_{l_{1}=0}^{\infty} \sum_{m_{1}=0}^{\infty} k_{1} P_{i_{1} j_{1} k_{1} l_{1} m_{1}} \\
& -k_{t r p} \sum_{i=1}^{\infty} \sum_{j=0}^{\infty} \sum_{k=0}^{\infty} \sum_{l=0}^{\infty} \sum_{m=0}^{\infty} k l^{2} P_{i j k l m} \sum_{i_{1}=1}^{\infty} \sum_{j_{1}=0}^{\infty} \sum_{k_{1}=0}^{\infty} \sum_{l_{1}=0}^{\infty} \sum_{m_{1}=0}^{\infty} i_{1} P_{i_{1} j_{1} k_{1} l_{1} m_{1}} \\
& -k_{t r p} \sum_{i=1}^{\infty} \sum_{j=0}^{\infty} \sum_{k=0}^{\infty} \sum_{l=0}^{\infty} \sum_{m=0}^{\infty} i l^{2} P_{i j k l m} \sum_{i_{1}=1}^{\infty} \sum_{j_{1}=0}^{\infty} \sum_{k_{1}=0}^{\infty} \sum_{l_{1}=0}^{\infty} \sum_{m_{1}=0}^{\infty} k_{1} P_{i_{1} j_{1}} k_{1} l_{1} m_{1} \\
& +k_{\operatorname{trp}} \sum_{i=1}^{\infty} \sum_{j=0}^{\infty} \sum_{k=0}^{\infty} \sum_{l=0}^{\infty} \sum_{m=0}^{\infty}(k+1) l^{2} P_{i j k+1 l m} \sum_{i_{1}=1}^{\infty} \sum_{j_{1}=0}^{\infty} \sum_{k_{1}=0}^{\infty} \sum_{l_{1}=0}^{\infty} \sum_{m_{1}=0}^{\infty} i_{1} P_{i_{1} j_{1} k_{1} l_{1} m_{1}} \\
& +k_{t r p} \sum_{i=1}^{\infty} \sum_{j=1}^{\infty} \sum_{k=1}^{\infty} \sum_{l=0}^{\infty} \sum_{m=0}^{\infty} i l^{2} P_{i j-1 k-1 l m} \sum_{i_{1}=1}^{\infty} \sum_{j_{1}=0}^{\infty} \sum_{k_{1}=0}^{\infty} \sum_{l_{1}=0}^{\infty} \sum_{m_{1}=0}^{\infty} k_{1} P_{i_{1} j_{1} k_{1} l_{1} m_{1}}
\end{aligned}
$$




$$
\begin{aligned}
& -k_{\operatorname{trp}} \sum_{i=1}^{\infty} \sum_{j=0}^{\infty} \sum_{k=0}^{\infty} \sum_{l=0}^{\infty} \sum_{m=0}^{\infty} l^{2} m P_{i j k l m} \sum_{i_{1}=1}^{\infty} \sum_{j_{1}=0}^{\infty} \sum_{k_{1}=0}^{\infty} \sum_{l_{1}=0}^{\infty} \sum_{m_{1}=0}^{\infty} i_{1} P_{i_{1} j_{1} k_{1} l_{1} m_{1}} \\
& -k_{t r p} \sum_{i=1}^{\infty} \sum_{j=0}^{\infty} \sum_{k=0}^{\infty} \sum_{l=0}^{\infty} \sum_{m=0}^{\infty} i l^{2} P_{i j k l m} \sum_{i_{1}=1}^{\infty} \sum_{j_{1}=0}^{\infty} \sum_{k_{1}=0}^{\infty} \sum_{l_{1}=0}^{\infty} \sum_{m_{1}=0}^{\infty} m_{1} P_{i_{1} j_{1} k_{1} l_{1} m_{1}} \\
& +k_{t r p} \sum_{i=1}^{\infty} \sum_{j=0}^{\infty} \sum_{k=0}^{\infty} \sum_{l=0}^{\infty} \sum_{m=0}^{\infty} l^{2}(m+1) P_{i j k l m+1} \sum_{i_{1}=1}^{\infty} \sum_{j_{1}=0}^{\infty} \sum_{k_{1}=0}^{\infty} \sum_{l_{1}=0}^{\infty} \sum_{m_{1}=0}^{\infty} i_{1} P_{i_{1} j_{1} k_{1} l_{1} m_{1}} \\
& +k_{t r p} \sum_{i=1}^{\infty} \sum_{j=1}^{\infty} \sum_{k=0}^{\infty} \sum_{l=0}^{\infty} \sum_{m=1}^{\infty} i l^{2} P_{i j-1 k l m-1} \sum_{i_{1}=1}^{\infty} \sum_{j_{1}=0}^{\infty} \sum_{k_{1}=0}^{\infty} \sum_{l_{1}=0}^{\infty} \sum_{m_{1}=0}^{\infty} m_{1} P_{i_{1} j_{1} k_{1} l_{1} m_{1}} \\
& -k_{\text {intr }} \sum_{i=1}^{\infty} \sum_{j=0}^{\infty} \sum_{k=0}^{\infty} \sum_{l=0}^{\infty} \sum_{m=0}^{\infty} k l^{2} P_{i j k l m} \\
& +k_{i n t r} \sum_{i=1}^{\infty} \sum_{j=1}^{\infty} \sum_{k=0}^{\infty} \sum_{l=0}^{\infty} \sum_{m=0}^{\infty} k l^{2} P_{i j-1 k l m} \\
& -k_{r} \sum_{i=1}^{\infty} \sum_{j=0}^{\infty} \sum_{k=0}^{\infty} \sum_{l=0}^{\infty} \sum_{m=0}^{\infty} l^{2} m P_{i j k l m} \\
& +k_{r} \sum_{i=1}^{\infty} \sum_{j=0}^{\infty} \sum_{k=1}^{\infty} \sum_{l=0}^{\infty} \sum_{m=0}^{\infty} l^{2}(m+1) P_{i j k-1 l m+1} \\
& -k_{t c} \sum_{i=1}^{\infty} \sum_{j=0}^{\infty} \sum_{k=0}^{\infty} \sum_{l=0}^{\infty} \sum_{m=0}^{\infty} l^{2} k P_{i j k l m} \sum_{i_{1}=1}^{\infty} \sum_{j_{1}=0}^{\infty} \sum_{k_{1}=0}^{\infty} \sum_{l_{1}=0}^{\infty} \sum_{m_{1}=0}^{\infty} k_{1} P_{i_{1} j_{1} k_{1} l_{1} m_{1}} \\
& +\frac{1}{2} k_{t c} \sum_{i=1}^{\infty} \sum_{j=0}^{\infty} \sum_{k=0}^{\infty} \sum_{l=0}^{\infty} \sum_{m=0}^{\infty} \sum_{i_{1}=1}^{i} \sum_{j_{1}=0}^{j} \sum_{k_{1}=0}^{k+2} \sum_{l_{1}=0}^{l} \sum_{m_{1}=0}^{m} l^{2}\left[k_{1} P_{i_{1} j_{1} k_{1} l_{1} m_{1}}\left(k+2-k_{1}\right) P_{i-i_{1}, j-j_{1}, k+2-k_{1}, l-l_{1} m-m_{1}}\right] \\
& -k_{t c} \sum_{i=1}^{\infty} \sum_{j=0}^{\infty} \sum_{k=0}^{\infty} \sum_{l=0}^{\infty} \sum_{m=0}^{\infty} l^{2} m P_{i j k l m} \sum_{i_{1}=1}^{\infty} \sum_{j_{1}=0}^{\infty} \sum_{k_{1}=0}^{\infty} \sum_{l_{1}=0}^{\infty} \sum_{m_{1}=0}^{\infty} m_{1} P_{i_{1} j_{1} k_{1} l_{1} m_{1}} \\
& +\frac{1}{2} k_{t c} \sum_{i=1}^{\infty} \sum_{j=0}^{\infty} \sum_{k=0}^{\infty} \sum_{l=0}^{\infty} \sum_{m=0}^{\infty} \sum_{i_{1}=1}^{i} \sum_{j_{1}=0}^{j} \sum_{k_{1}=0}^{k} \sum_{l_{1}=0}^{l} \sum_{m_{1}=0}^{m+2}\left[l^{2} m_{1} P_{i_{1} j_{1} k_{1} l_{1} m_{1}}\left(m+2-m_{1}\right) P_{i-i_{1}, j-j_{1}, k-k_{1}, l-l_{1} m+2-m_{1}}\right] \\
& -k_{t c} \sum_{i=1}^{\infty} \sum_{j=0}^{\infty} \sum_{k=0}^{\infty} \sum_{l=0}^{\infty} \sum_{m=0}^{\infty} k l^{2} P_{i j k l m} \sum_{i_{1}=1}^{\infty} \sum_{j_{1}=0}^{\infty} \sum_{k_{1}=0}^{\infty} \sum_{l_{1}=0}^{\infty} \sum_{m_{1}=0}^{\infty} m_{1} P_{i_{1} j_{1} k_{1} l_{1} m_{1}} \\
& -k_{t c} \sum_{i=1}^{\infty} \sum_{j=0}^{\infty} \sum_{k=0}^{\infty} \sum_{l=0}^{\infty} \sum_{m=0}^{\infty} l^{2} m P_{i j k l m} \sum_{i_{1}=1}^{\infty} \sum_{j_{1}=0}^{\infty} \sum_{k_{1}=0}^{\infty} \sum_{l_{1}=0}^{\infty} \sum_{m_{1}=0}^{\infty} k_{1} P_{i_{1} j_{1} k_{1} l_{1} m_{1}} \\
& +\frac{1}{2} k_{t c} \sum_{i=1}^{\infty} \sum_{j=0}^{\infty} \sum_{k=0}^{\infty} \sum_{l=0}^{\infty} \sum_{m=0}^{\infty} \sum_{i_{1}=1}^{i} \sum_{j_{1}=0}^{j} \sum_{k_{1}=0}^{k+1} \sum_{l_{1}=0}^{l} \sum_{m_{1}=0}^{m+1}\left[l^{2} k_{1} P_{i_{1} j_{1} k_{1} l_{1} m_{1}}\left(m+1-m_{1}\right) P_{\left.i-i_{1}, j-j_{1}, k+1-k_{1}, l-l_{1} m+1-m_{1}\right]}\right. \\
& +\frac{1}{2} k_{t c} \sum_{i=1}^{\infty} \sum_{j=0}^{\infty} \sum_{k=0}^{\infty} \sum_{l=0}^{\infty} \sum_{m=0}^{\infty} \sum_{i_{1}=1}^{i} \sum_{j_{1}=0}^{j} \sum_{k_{1}=0}^{k+1} \sum_{l_{1}=0}^{l} \sum_{m_{1}=0}^{m+1}\left[l^{2} m_{1} P_{i_{1} j_{1} k_{1} l_{1} m_{1}}\left(k+1-k_{1}\right) P_{i-i_{1}, j-j_{1}, k+1-k_{1}, l-l_{1} m+1-m_{1}}\right]
\end{aligned}
$$




$$
\begin{aligned}
& \Rightarrow \frac{d \mu_{00020}}{d t}=-k_{p} C_{M} \sum_{i=1}^{\infty} \sum_{j=0}^{\infty} \sum_{k=0}^{\infty} \sum_{l=0}^{\infty} \sum_{m=0}^{\infty} k l^{2} P_{i j k l m} \\
& +k_{p} C_{M} \sum_{i=1}^{\infty} \sum_{j=0}^{\infty} \sum_{k=0}^{\infty} \sum_{l=0}^{\infty} \sum_{m=0}^{\infty} k l^{2} P_{i j k l m} \\
& -k_{p} C_{M} \sum_{i=1}^{\infty} \sum_{j=0}^{\infty} \sum_{k=0}^{\infty} \sum_{l=0}^{\infty} \sum_{m=0}^{\infty} l^{2} m P_{i j k l m} \\
& +k_{p} C_{M} \sum_{i=1}^{\infty} \sum_{j=0}^{\infty} \sum_{k=0}^{\infty} \sum_{l=0}^{\infty} \sum_{m=0}^{\infty} l^{2} m P_{i j k l m} \\
& -k_{t r t} C_{T} \sum_{i=1}^{\infty} \sum_{j=0}^{\infty} \sum_{k=0}^{\infty} \sum_{l=0}^{\infty} \sum_{m=0}^{\infty} k l^{2} P_{i j k l m} \\
& +k_{t r t} C_{T} \sum_{i=1}^{\infty} \sum_{j=0}^{\infty} \sum_{k=0}^{\infty} \sum_{l=0}^{\infty} \sum_{m=0}^{\infty} k(l+1)^{2} P_{i j k l m} \\
& -k_{t r t} \sum_{i=1}^{\infty} \sum_{j=0}^{\infty} \sum_{k=0}^{\infty} \sum_{l=0}^{\infty} \sum_{m=0}^{\infty} k l^{2} P_{i j k l m} \sum_{i_{1}=1}^{\infty} \sum_{j_{1}=0}^{\infty} \sum_{k_{1}=0}^{\infty} \sum_{l_{1}=0}^{\infty} \sum_{m_{1}=0}^{\infty} l_{1} P_{i_{1} j_{1} k_{1} l_{1} m_{1}} \\
& -k_{\text {trt }} \sum_{i=1}^{\infty} \sum_{j=0}^{\infty} \sum_{k=0}^{\infty} \sum_{l=0}^{\infty} \sum_{m=0}^{\infty} l^{3} P_{i j k l m} \sum_{i_{1}=1}^{\infty} \sum_{j_{1}=0}^{\infty} \sum_{k_{1}=0}^{\infty} \sum_{l_{1}=0}^{\infty} \sum_{m_{1}=0}^{\infty} k_{1} P_{i_{1} j_{1} k_{1} l_{1} m_{1}} \\
& +k_{t r t} \sum_{i=1}^{\infty} \sum_{j=0}^{\infty} \sum_{k=0}^{\infty} \sum_{l=0}^{\infty} \sum_{m=0}^{\infty} k(l+1)^{2} P_{i j k l m} \sum_{i_{1}=1}^{\infty} \sum_{j_{1}=0}^{\infty} \sum_{k_{1}=0}^{\infty} \sum_{l_{1}=0}^{\infty} \sum_{m_{1}=0}^{\infty} l_{1} P_{i_{1} j_{1} k_{1} l_{1} m_{1}} \\
& +k_{t r t} \sum_{i=1}^{\infty} \sum_{j=0}^{\infty} \sum_{k=0}^{\infty} \sum_{l=0}^{\infty} \sum_{m=0}^{\infty}(l-1)^{2} l P_{i j k l m} \sum_{i_{1}=1}^{\infty} \sum_{j_{1}=0}^{\infty} \sum_{k_{1}=0}^{\infty} \sum_{l_{1}=0}^{\infty} \sum_{m_{1}=0}^{\infty} k_{1} P_{i_{1} j_{1} k_{1} l_{1} m_{1}} \\
& -k_{t r p} \sum_{i=1}^{\infty} \sum_{j=0}^{\infty} \sum_{k=0}^{\infty} \sum_{l=0}^{\infty} \sum_{m=0}^{\infty} k l^{2} P_{i j k l m} \sum_{i_{1}=1}^{\infty} \sum_{j_{1}=0}^{\infty} \sum_{k_{1}=0}^{\infty} \sum_{l_{1}=0}^{\infty} \sum_{m_{1}=0}^{\infty} i_{1} P_{i_{1} j_{1} k_{1} l_{1} m_{1}} \\
& -k_{t r p} \sum_{i=1}^{\infty} \sum_{j=0}^{\infty} \sum_{k=0}^{\infty} \sum_{l=0}^{\infty} \sum_{m=0}^{\infty} i l^{2} P_{i j k l m} \sum_{i_{1}=1}^{\infty} \sum_{j_{1}=0}^{\infty} \sum_{k_{1}=0}^{\infty} \sum_{l_{1}=0}^{\infty} \sum_{m_{1}=0}^{\infty} k_{1} P_{i_{1} j_{1} k_{1} l_{1} m_{1}} \\
& +k_{t r p} \sum_{i=1}^{\infty} \sum_{j=0}^{\infty} \sum_{k=0}^{\infty} \sum_{l=0}^{\infty} \sum_{m=0}^{\infty} k l^{2} P_{i j k l m} \sum_{i_{1}=1}^{\infty} \sum_{j_{1}=0}^{\infty} \sum_{k_{1}=0}^{\infty} \sum_{l_{1}=0}^{\infty} \sum_{m_{1}=0}^{\infty} i_{1} P_{i_{1} j_{1} k_{1} l_{1} m_{1}} \\
& +k_{t r p} \sum_{i=1}^{\infty} \sum_{j=0}^{\infty} \sum_{k=0}^{\infty} \sum_{l=0}^{\infty} \sum_{m=0}^{\infty} i l^{2} P_{i j k l m} \sum_{i_{1}=1}^{\infty} \sum_{j_{1}=0}^{\infty} \sum_{k_{1}=0}^{\infty} \sum_{l_{1}=0}^{\infty} \sum_{m_{1}=0}^{\infty} k_{1} P_{i_{1} j_{1} k_{1} l_{1} m_{1}}
\end{aligned}
$$




$$
\begin{aligned}
& -k_{\operatorname{trp}} \sum_{i=1}^{\infty} \sum_{j=0}^{\infty} \sum_{k=0}^{\infty} \sum_{l=0}^{\infty} \sum_{m=0}^{\infty} l^{2} m P_{i j k l m} \sum_{i_{1}=1}^{\infty} \sum_{j_{1}=0}^{\infty} \sum_{k_{1}=0}^{\infty} \sum_{l_{1}=0}^{\infty} \sum_{m_{1}=0}^{\infty} i_{1} P_{i_{1} j_{1} k_{1} l_{1} m_{1}} \\
& -k_{\operatorname{trp}} \sum_{i=1}^{\infty} \sum_{j=0}^{\infty} \sum_{k=0}^{\infty} \sum_{l=0}^{\infty} \sum_{m=0}^{\infty} i l^{2} P_{i j k l m} \sum_{i_{1}=1}^{\infty} \sum_{j_{1}=0}^{\infty} \sum_{k_{1}=0}^{\infty} \sum_{l_{1}=0}^{\infty} \sum_{m_{1}=0}^{\infty} m_{1} P_{i_{1} j_{1} k_{1} l_{1} m_{1}} \\
& +k_{\text {trp }} \sum_{i=1}^{\infty} \sum_{j=0}^{\infty} \sum_{k=0}^{\infty} \sum_{l=0}^{\infty} \sum_{m=0}^{\infty} l^{2} m P_{i j k l m} \sum_{i_{1}=1}^{\infty} \sum_{j_{1}=0}^{\infty} \sum_{k_{1}=0}^{\infty} \sum_{l_{1}=0}^{\infty} \sum_{m_{1}=0}^{\infty} i_{1} P_{i_{1} j_{1} k_{1} l_{1} m_{1}} \\
& +k_{\operatorname{trp}} \sum_{i=1}^{\infty} \sum_{j=0}^{\infty} \sum_{k=0}^{\infty} \sum_{l=0}^{\infty} \sum_{m=0}^{\infty} i l^{2} P_{i j k l m} \sum_{i_{1}=1}^{\infty} \sum_{j_{1}=0}^{\infty} \sum_{k_{1}=0}^{\infty} \sum_{l_{1}=0}^{\infty} \sum_{m_{1}=0}^{\infty} m_{1} P_{i_{1} j_{1} k_{1} l_{1} m_{1}} \\
& -k_{\text {intr }} \sum_{i=1}^{\infty} \sum_{j=0}^{\infty} \sum_{k=0}^{\infty} \sum_{l=0}^{\infty} \sum_{m=0}^{\infty} k l^{2} P_{i j k l m} \\
& +k_{\text {intr }} \sum_{i=1}^{\infty} \sum_{j=0}^{\infty} \sum_{k=0}^{\infty} \sum_{l=0}^{\infty} \sum_{m=0}^{\infty} k l^{2} P_{i j k l m} \\
& -k_{r} \sum_{i=1}^{\infty} \sum_{j=0}^{\infty} \sum_{k=0}^{\infty} \sum_{l=0}^{\infty} \sum_{m=0}^{\infty} l^{2} m P_{i j k l m} \\
& +k_{r} \sum_{i=1}^{\infty} \sum_{j=0}^{\infty} \sum_{k=1}^{\infty} \sum_{l=0}^{\infty} \sum_{m=0}^{\infty} l^{2} m P_{i j k l m} \\
& -k_{t c} \sum_{i=1}^{\infty} \sum_{j=0}^{\infty} \sum_{k=0}^{\infty} \sum_{l=0}^{\infty} \sum_{m=0}^{\infty} k l^{2} P_{i j k l m} \sum_{i_{1}=1}^{\infty} \sum_{j_{1}=0}^{\infty} \sum_{k_{1}=0}^{\infty} \sum_{l_{1}=0}^{\infty} \sum_{m_{1}=0}^{\infty} k_{1} P_{i_{1} j_{1} k_{1} l_{1} m_{1}} \\
& +\frac{1}{2}(2) k_{t c} \sum_{i=1}^{\infty} \sum_{j=0}^{\infty} \sum_{k=0}^{\infty} \sum_{l=0}^{\infty} \sum_{m=0}^{\infty} k l^{2} P_{i j k l m} \sum_{i_{1}=1}^{\infty} \sum_{j_{1}=0}^{\infty} \sum_{k_{1}=0}^{\infty} \sum_{l_{1}=0}^{\infty} \sum_{m_{1}=0}^{\infty} k_{1} P_{i_{1} j_{1} k_{1} l_{1} m_{1}} \\
& +\frac{1}{2}(2) k_{t c} \sum_{i=1}^{\infty} \sum_{j=0}^{\infty} \sum_{k=0}^{\infty} \sum_{l=0}^{\infty} \sum_{m=0}^{\infty} k l P_{i j k l m} \sum_{i_{1}=1}^{\infty} \sum_{j_{1}=0}^{\infty} \sum_{k_{1}=0}^{\infty} \sum_{l_{1}=0}^{\infty} \sum_{m_{1}=0}^{\infty} k_{1} l_{1} P_{i_{1} j_{1} k_{1} l_{1} m_{1}} \\
& -k_{t c} \sum_{i=1}^{\infty} \sum_{j=0}^{\infty} \sum_{k=0}^{\infty} \sum_{l=0}^{\infty} \sum_{m=0}^{\infty} l^{2} m P_{i j k l m} \sum_{i_{1}=1}^{\infty} \sum_{j_{1}=0}^{\infty} \sum_{k_{1}=0}^{\infty} \sum_{l_{1}=0}^{\infty} \sum_{m_{1}=0}^{\infty} m_{1} P_{i_{1} j_{1} k_{1} l_{1} m_{1}} \\
& +\frac{1}{2}(2) k_{t c} \sum_{i=1}^{\infty} \sum_{j=0}^{\infty} \sum_{k=0}^{\infty} \sum_{l=0}^{\infty} \sum_{m=0}^{\infty} l^{2} m P_{i j k l m} \sum_{i_{1}=1}^{\infty} \sum_{j_{1}=0}^{\infty} \sum_{k_{1}=0}^{\infty} \sum_{l_{1}=0}^{\infty} \sum_{m_{1}=0}^{\infty} m_{1} P_{i_{1} j_{1} k_{1} l_{1} m_{1}} \\
& +\frac{1}{2} k_{t c} \sum_{i=1}^{\infty} \sum_{j=0}^{\infty} \sum_{k=0}^{\infty} \sum_{l=0}^{\infty} \sum_{m=0}^{\infty} \operatorname{lm} P_{i j k l m} \sum_{i_{1}=1}^{\infty} \sum_{j_{1}=0}^{\infty} \sum_{k_{1}=0}^{\infty} \sum_{l_{1}=0}^{\infty} \sum_{m_{1}=0}^{\infty} l_{1} m_{1} P_{i_{1} j_{1} k_{1} l_{1} m_{1}}
\end{aligned}
$$




$$
\begin{aligned}
& -k_{t c} \sum_{i=1}^{\infty} \sum_{j=0}^{\infty} \sum_{k=0}^{\infty} \sum_{l=0}^{\infty} \sum_{m=0}^{\infty} k l^{2} P_{i j k l m} \sum_{i_{1}=1}^{\infty} \sum_{j_{1}=0}^{\infty} \sum_{k_{1}=0}^{\infty} \sum_{l_{1}=0}^{\infty} \sum_{m_{1}=0}^{\infty} m_{1} P_{i_{1} j_{1} k_{1} l_{1} m_{1}} \\
& -k_{t c} \sum_{i=1}^{\infty} \sum_{j=0}^{\infty} \sum_{k=0}^{\infty} \sum_{l=0}^{\infty} \sum_{m=0}^{\infty} l^{2} m P_{i j k l m} \sum_{i_{1}=1}^{\infty} \sum_{j_{1}=0}^{\infty} \sum_{k_{1}=0}^{\infty} \sum_{l_{1}=0}^{\infty} \sum_{m_{1}=0}^{\infty} k_{1} P_{i_{1} j_{1} k_{1} l_{1} m_{1}} \\
& +\frac{1}{2}(2) k_{t c} \sum_{i=1}^{\infty} \sum_{j=0}^{\infty} \sum_{k=0}^{\infty} \sum_{l=0}^{\infty} \sum_{m=0}^{\infty} k l^{2} P_{i j k l m} \sum_{i_{1}=1}^{\infty} \sum_{j_{1}=0}^{\infty} \sum_{k_{1}=0}^{\infty} \sum_{l_{1}=0}^{\infty} \sum_{m_{1}=0}^{\infty} m_{1} P_{i_{1} j_{1} k_{1} l_{1} m_{1}} \\
& +\frac{1}{2}(2) k_{t c} \sum_{i=1}^{\infty} \sum_{j=0}^{\infty} \sum_{k=0}^{\infty} \sum_{l=0}^{\infty} \sum_{m=0}^{\infty} l^{2} m P_{i j k l m} \sum_{i_{1}=1}^{\infty} \sum_{j_{1}=0}^{\infty} \sum_{k_{1}=0}^{\infty} \sum_{l_{1}=0}^{\infty} \sum_{m_{1}=0}^{\infty} k_{1} P_{i_{1} j_{1} k_{1} l_{1} m_{1}} \\
& +\frac{1}{2}(4) k_{t c} \sum_{i=1}^{\infty} \sum_{j=0}^{\infty} \sum_{k=0}^{\infty} \sum_{l=0}^{\infty} \sum_{m=0}^{\infty} k l P_{i j k l m} \sum_{i_{1}=1}^{\infty} \sum_{j_{1}=0}^{\infty} \sum_{k_{1}=0}^{\infty} \sum_{l_{1}=0}^{\infty} \sum_{m_{1}=0}^{\infty} l_{1} m_{1} P_{i_{1} j_{1} k_{1} l_{1} m_{1}}
\end{aligned}
$$

using Equations (67) and (68)

$$
\begin{aligned}
& \Rightarrow \frac{d \mu_{00020}}{d t}=k_{t r t} C_{T} \sum_{i=1}^{\infty} \sum_{j=0}^{\infty} \sum_{k=0}^{\infty} \sum_{l=0}^{\infty} \sum_{m=0}^{\infty}(2 k l+k) P_{i j k l m} \\
& \quad+k_{t r t} \sum_{i=1}^{\infty} \sum_{j=0}^{\infty} \sum_{k=0}^{\infty} \sum_{l=0}^{\infty} \sum_{m=0}^{\infty}(2 k l+k) P_{i j k l m} \sum_{i_{1}=1}^{\infty} \sum_{j_{1}=0}^{\infty} \sum_{k_{1}=0}^{\infty} \sum_{l_{1}=0}^{\infty} \sum_{m_{1}=0}^{\infty} l_{1} P_{i_{1} j_{1} k_{1} l_{1} m_{1}} \\
& \quad+k_{t r t} \sum_{i=1}^{\infty} \sum_{j=0}^{\infty} \sum_{k=0}^{\infty} \sum_{l=0}^{\infty} \sum_{m=0}^{\infty}\left(l-2 l^{2}\right) P_{i j k l m} \sum_{i_{1}=1}^{\infty} \sum_{j_{1}=0}^{\infty} \sum_{k_{1}=0}^{\infty} \sum_{l_{1}=0}^{\infty} \sum_{m_{1}=0}^{\infty} k_{1} P_{i_{1} j_{1} k_{1} l_{1} m_{1}} \\
& \quad+k_{t c} \sum_{i=1}^{\infty} \sum_{j=0}^{\infty} \sum_{k=0}^{\infty} \sum_{l=0}^{\infty} \sum_{m=0}^{\infty} k l P_{i j k l m} \sum_{i_{1}=1}^{\infty} \sum_{j_{1}=0}^{\infty} \sum_{k_{1}=0}^{\infty} \sum_{l_{1}=0}^{\infty} \sum_{m_{1}=0}^{\infty} k_{1} l_{1} P_{i_{1} j_{1} k_{1} l_{1} m_{1}} \\
& \quad+k_{t c} \sum_{i=1}^{\infty} \sum_{j=0}^{\infty} \sum_{k=0}^{\infty} \sum_{l=0}^{\infty} \sum_{m=0}^{\infty} l m P_{i j k l m} \sum_{i_{1}=1}^{\infty} \sum_{j_{1}=0}^{\infty} \sum_{k_{1}=0}^{\infty} \sum_{l_{1}=0}^{\infty} \sum_{m_{1}=0}^{\infty} l_{1} m_{1} P_{i_{1} j_{1} k_{1} l_{1} m_{1}} \\
& \quad+2 k_{t c} \sum_{i=1}^{\infty} \sum_{j=0}^{\infty} \sum_{k=0}^{\infty} \sum_{l=0}^{\infty} \sum_{m=0}^{\infty} k l P_{i j k l m} \sum_{i_{1}=1}^{\infty} \sum_{j_{1}=0}^{\infty} \sum_{k_{1}=0}^{\infty} \sum_{l_{1}=0}^{\infty} \sum_{m_{1}=0}^{\infty} l_{1} m_{1} P_{i_{1} j_{1} k_{1} l_{1} m_{1}} \\
& \Rightarrow \frac{d \mu_{00020}}{d t}=k_{t r t}\left(C_{T}+\mu_{00010}\right)\left(2 \mu_{00110}+\mu_{00100}\right)+k_{t r t} \mu_{00100}\left(\mu_{00010}-2 \mu_{00020}\right) \\
& +k_{t c}\left(\mu_{00110}\right)^{2}+k_{t c}\left(\mu_{00011}\right)^{2}+2 k_{t c} \mu_{00110} \mu_{00011}
\end{aligned}
$$

